\title{
Quantum Gravity Emergence from Entanglement in a Multi-Fold Universe
}

\author{
Stephane H. Maes
}

shmaes.physics@gmail.com

\begin{abstract}
We start from a hypothetical multi-fold universe $U_{M F}$, where the propagation of everything is slower or equal to the speed of light and where entanglement extends the set of paths available to Path Integrals. This multifold mechanism enables EPR (Einstein-Podolsky-Rosen) "spooky actions at distance" to result from local interactions in the resulting folds. It produces gravity-like attractive effective potentials in the spacetime, between entangled entities, that are caused by the curvature of the folds. When quantized, multi-folds correspond to gravitons and they are enablers of EPR entanglement. Gravity emerges nonperturbative and covariant from EPR entanglement between virtual particles surrounding an entity. In $U_{M F}$, we encounter mechanisms that predict gravity fluctuations when entanglement is present, including in macroscopic entanglements. Besides providing a new perspective on quantum gravity, when added to the Standard Model as $\left(\mathrm{SM}_{\mathrm{G}}\right)$, with non-negligible affects at its scales, and to the Standard Cosmology, $U_{M F}$ can contribute explanations of several open questions and challenges. It also clarifies some relationships and challenges met by other quantum gravity models and Theories of Everything. It leads to suggestions for these works. We also reconstruct the spacetime of $U_{M F}$, starting from the random walks of particles in an early spacetime. $U_{M F}$ now appears as a noncommutative, discrete, yet Lorentz symmetric, spacetime that behaves roughly 2-Dimensional at Planck scales, when it is a graph of microscopic Planck size black holes on a random walk fractal structure left by particles that can also appear as microscopic black holes. Of course, at larger scales, spacetime appears 4-D, where we are able to explain curvature and recover Einstein's General Relativity. We also discover an entanglement gravity-like contributions and massive gravity at very small scales. This is remarkable considering that no Hilbert Einstein action, or variations expressing area invariance, were introduced. Our model also explains why semi classical approaches can work till way smaller scale than usually expected and present a new view on an Ultimate Unification of all forces, at very small scales. We also explore opportunities for falsifiability and validation of our model, as well as ideas for futuristic applications, that may be worth considering, if $U_{M F}$ was a suitable model for our universe $U_{\text {real }}$.
\end{abstract}

Keywords: Quantum Gravity; Quantum Entanglement; Multi-fold Universe; Standard Model with Gravity $\left(\mathrm{SM}_{\mathrm{G}}\right)$

\section{INTRODUCTION}

This paper presents a radically new analysis of the foundations of quantum physics and quantum gravity. It is not just following a constructive path of spacetime or quantum geometry. It is not just "not thinking and instead computing" Path Integrals with variational approaches, Feynman diagrams or lattice models of Actions, Lagrangian or Hamiltonians, derived or guessed from Hilbert-Einstein actions and geometric considerations or analogies. It is not starting from linearizing or quantizing General Relativity (GR) equations at small scales.

It is not attempting to deal with divergences and renormalization by further tweaking the Action, Hamiltonian or Lagrangian and then claiming victory when recovering GR and gravitons (as spin 2 bosons), or vice versa, or spacetime thermodynamics baked in all along because of the commonalities between an Action and the Hilbert Einstein Action.

This paper may not immediately appear to follow a reasoning or formalism familiar to today's physicists. It is not because

Cite as: Stephane H. Maes, (2020-2022) “Quantum Gravity Emergence from Entanglement in a Multi-Fold Universe”, HIJ, Vol 2, No 4, pp 136-219, Dec 2022, https://doi.org/10.55672/hij2022pp136-219, https://shmaesphysics.wordpress.com/2020/06/09/paper-published-as-preprint-quantum-gravity-emergencefrom-entanglement-in-a-multi-fold-universe/, and viXra:2006.0088 - https://vixra.org/abs/2006.0088, (June 9, 2020).

HTML is at https://shmaesphysics.wordpress.com/2022/11/09/quantum-gravity-emergence-from-entanglement-in-a-multi-fold-universe-2/ 
the more well beaten paths are not the right ways to go; to the contrary. It is rather that, lately, many have called out "physics in crisis" and "the need for a new physics" [236, 237]. Today, it is something it is no more just the topic of only scientific articles, discussions at physics conferences or deep within physics departments.

Discussions of the challenges have reached much wider audience articles and publications (e.g., [1, 248, 236]). The list of the issues warranting new approaches include, nonexhaustively: the un-intuitiveness of quantum physics with interpretations that are often esoteric and hard to follow; the problems with mathematics as sole driver for theoretical physics progress along with the loss of falsifiability[238], and absence of validated new physics in last few decades [236]; the frustrating long marches to merge, or maybe just position, General Relativity and Quantum Physics including as a result the absence of an unambiguous quantum theory of gravity [2], or all-encompassing grand unification ${ }^{1-2}$, or related "Theories of Everything"; the lack of explanation for dark matter and dark energy [3], or particle/anti-particle imbalance; the conflicting observations in terms of cosmology and the universe expansion that is accelerating (too fast), as well as in terms of its early inflation [1]. To this, we should add the religious wars between schools of thoughts on how to target quantum gravity [237]. We thought that something a bit more radical may be worth attempting.

Instead of spending time trying to map our theory onto wellestablished frameworks, we decided to pursue a thought process, inspired by a few first principles and considerations intersecting General Relativity and Quantum Physics, and introduce a universe $U_{M F}$ where Physics applies as usual ${ }^{3}$ but where some additional quantitative, qualitative, phenomenological and mathematical features are added, combined and pursued. This way, we hope to address some of the challenges discussed above; hoping that emerging mechanisms can explain aspects of these challenges. The approach that we follow seems justified, because, today, Physics seems to lack something to leap over its current stumbling blocks. And yes, doing so, we maybe escape the sirens of theoretical physics based only on progressing always the same way with an aesthetically pleasant enough program, and all its rigor. The price to pay is that we do not have yet the complete formalism to express or derive everything. We try to be revolutionary, provocative and to address heads on what we think are some the main irreconcilable differences between GR and Quantum Physics. Yet, we also try to stay connected and consistent with the established Physics as well as some of the latest trends in Physics. The intent is to revisit and model all aspects more rigorously in upcoming works rigorously in upcoming works (See [513] for the latest developments and track all the related papers).

Our work has not benefited from living and breathing Physics, tracking and discussing trends and new papers or attending conferences over the years. It relies extensively on occasional updates about some of the latest fads and publications. For the rest it is the result from an old intuition, that Feynman's Path Integrals and Actions are the most fundamental formalisms of Physics (like an "equation of God", and we are not talking of Euler's formula), and that EPR (Einstein-Podolsky-Rosen) entanglement [4, 5] and related works, and implications around the Bell inequalities [265], are fundamental and at the center of something still only partially understood.

In hindsight, (EPR) entanglement is today at the core of the most non-classical quantum phenomena and defines Quantum Physics. Quantum entanglement is also the foundation of quantum computing and Qubits [ㅁ, 7]]. The essence of the incompatibilities of GR and Quantum Physics relate to local realism vs. quantum nonlocality $[\underline{5}, \underline{265}]$ and superposition ${ }^{4}$. Also, Feynman's integral or more generally functional integrals involving Actions and Lagrangian or Hamiltonians formulations are behind most ${ }^{5}$ modern physics theories [322]. The reason for the existence of Actions (locally extremized or equivalently locally invariant) in Physics (classical or quantum) remains a wonder: why is it possible to capture complex dynamical models (histories or trajectories) simply in a concise extremization of an Action equation $[322]^{6}$ ? Physical Actions give rise to most of modern physics models (Dynamics and Kinematics).

Our hypotheses slowly developed over the years, linking the Path Integrals and EPR together as a reason why spacetime would curve and gravity would result from the need to support Einstein's "spooky action at distance" of EPR despite $c$ limits.

Popular discussions of Verlinde's work on gravity as an entropic force [10] actually emerging from entanglement entropy $[11, \underline{12}, \underline{13}, \underline{14}]$ created the motivation to study the details and publish the ideas. Similar keywords in [10] made it sound like this was our concept all along. However, while similar at face value, it turns out that Verlinde's work is quite different from what is proposed here. The thought processes are also radically different. Instead of a statistical (hence entropy friendly) derivation of gravity due to field entanglement (in the bulk) of spacetime and at the surface of spacetime, as proposed by [14], our paper proposes both a macroscopic and a microscopic behavior of spacetime (in $U_{M F}$ ) as a result of, or to support, specific microscopic (i.e., not a just statistical average), EPR entanglements. In our model, gravity-like effective potentials (as well as effective spacetime curvatures) emerge from these entanglements, when computing Path Integrals in $U_{M F}$ for EPR entangled systems.

Assuming that the model presented here would characterize correctly our universe's $U_{\text {real }}$ spacetime, then by pursuing the consequences of the model both at the macroscopic and at the microscopic level, we can predict or derive the existence of new phenomena and the behavior of several phenomena involved in some of Today's Physics stumbling blocks. We also recover, connect, or put in perspective hypotheses and works that have been developed over the years. . Interestingly it also allows us to understand possible gaps in some of the most successful (or promising) models of Physics like QFT (with the second quantization and unfixed number of particles ${ }^{7}$ ) $[\underline{15}, \underline{16}, \underline{17}], \mathbf{G R}$ (as mostly a Thermodynamic theory of spacetime when trying to understand its view on microscopic structure) [18] and 
quantum gravity (e.g. String theory $[\underline{19}, \underline{20}]$, LQG (Loop Quantum Gravity, and more) [21] and other spacetime construction theories derived or constructed from Regge Calculus [22, 23, 243]). Indeed, in $U_{M F}$, we will see that EPR entanglement is responsible for gravity. In order to progress at the microscopic level (i.e., with quantum gravity), other theories must also be able to model individual entanglements.

It also means adopting particle tracking (e.g. See [24, 25] as examples of ways to address the challenges discussed in $[15$, $\underline{26}$ ), and adding particle-specific (instead of statistical [27, 242]/second quantization based) models of entanglement.

Our work may also provide a new twist, via different motivations, to the AdS/CFT correspondence conjecture $[\underline{29}, \underline{28}]$, holographic approaches to gravity $[\underline{29}, \underline{28}, \underline{30}, \underline{31}]$ and the Area laws of entropy for Black Holes and spacetime horizons in general $[\underline{18}, \underline{32}, \underline{33}, \underline{34}]$ as well as analyses of criteria with respect to the Swampland [61]. We will see that we recover the ADS/CFT correspondence conjecture, with (renormalizable) QFT on one side, and gravitons in $\mathbf{A d S}(5)$ on the other. In $U_{M F}$, the correspondence is not just a conjecture anymore. In addition, we will derive that gravity emerging this way is in fact renormalizable ${ }^{8}$ in the background spacetime. For example, we will see that superstrings would make a lot of sense in $\mathbf{A d S}(5)\left(\times S^{5}(+1\right.$ more for M-theory)) rather than any other type of universe; especially not our spacetime with its positive curvature.

$U_{M F}$ reconstructions from a situation where no spacetime or no particle exist will lead to a fractal discrete spacetime at Planck scale and models compatible with GR as the scale of analysis is increased (including a semi classical validity to way smaller scale than usually expected).

It sheds new lights on inflation, Path Integral formalism and the understanding of gravitons (real, virtual, massless and massive). We will also see examples of impacts in the context of the Standard Model. The formulation presented in this paper is still in its infancy in terms of its mathematical framework (e.g. actual Action, Lagrangian or Hamiltonian and quantitative estimates/coupling constants) which is for further works.

We mostly build the framework by coalescing previous works, using them to derive or prove statements and reinterpreting them in our context. As a first step, we obtain several phenomenological and qualitative predictions that can help validate or invalidate the approach.

Yet not only do we derive guidance for evolutions of many on-going works, if they were to apply in $U_{M F}$; but we also obtain results compatible with these works, usually more generic or with tantalizing twists; as if hinting at deltas in interpretations, complementarities, extensions or subtle contributions worth investigating within the respective frameworks.

In this context, it is important to state the following clearly. A major difference between our paper and most of the literature focused on quantum gravity is that we do not start from Einstein's GR field equations, or the Hilbert-Einstein action $^{9}$ (explicitly or implicitly through deficit angle or area extremization, or other manipulation of the Action). Instead, we start from Einstein's special relativity $c$ limit, interpreted more restrictively than usually, and an interpretation of EPR entanglement that reconciles the irreconcilable: locality with non-locality. Doing so, we also interpret the $\mathbf{G R}=\mathbf{Q M}[\underline{49}$ ] and $\mathbf{E R}=\mathbf{E P R}[\underline{86}, \underline{50}]$ conjectures and other related conjectures that we discovered while collecting a list of works related to this paper. In our view, these conjectures are built on the right intuitions, but they were not proposed as answers to all what we see as relevant, and, therefore they have not been fully pursued (yet) where all these ideas could have led them.

\subsection{Setting up the stage}

Post General Relativity (GR), Einstein spent much of his life trying to converge or unify General Relativity, Electromagnetism and Quantum Mechanics while doubting in particular the completeness and consistency of the latter. In his quest, Einstein aimed at also showing the incompatibilities of these different theories. In particular, he proposed the EPR (Einstein-Podolsky-Rosen) paradox to demonstrate the inconsistencies and incompleteness of Quantum Physics [4]. EPR remained an esoteric topic outside the Physics community until recently. But it has all changed with the recent developments of quantum computing. Few physical phenomena are as important as quantum entanglement and the associated EPR paradoxes and Bell inequalities violations $[\underline{4}, \underline{5}, \underline{265}]$; especially when it comes understanding the differences between classical and quantum physics.

The development of Quantum Field Theory (QFT) and its Quantum Electrodynamic (QED), Quantum

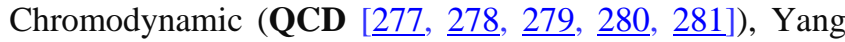
Mills, Gauge and Electroweak/Englert-Brout-HiggsGuralnik-Hagen-Kibble mechanism theories took precedence for a while, and led to the successful Standard Model, repeatedly experimentally confirmed [35, 36, 37] . Focus on the unification quest and dream followed the crowing of the Standard Model (SM), and evolved into the still much less fruitful, endeavours of Grand Unifications [38], Super Symmetry [39], Super Strings theories (including, in particular, the mysterious, and still essentially undefined, M-Theory) [19, 20], theories of everything (ToE) [40] and quantum gravity formulations (e.g. superstring theory, Loop Quantum Gravity (LQG) and Causal Dynamic triangulation or Lattice Gravity, and more) $[\underline{20}, \underline{2}, \underline{41}, \underline{42}, \underline{21}$, $\underline{22}, \underline{243}]$. Indeed, none of these theories have managed to overcome the problems with incompleteness, making unconfirmed predictions or even appearing unfalsifiable $[\underline{236}, \underline{239}, \underline{240}, \underline{237}, \underline{238}]^{10}$. It certainly also does not help that quantizing the gravity field or General Relativity does not seem renormalizable $[\underline{43}, \underline{44}, \underline{23}, \underline{59}, \underline{60}]$ and opens tricky unitarity questions beyond renormalization [45, 59].

In this paper, we do not start with an attempt to model apriori gravity or spacetime; we will do some of it in a later section using our earlier findings. Instead, we start by postulate a few axioms or principles that absolutely forbid supra luminosity and we start from Quantum Physics formulated with Path Integrals [46], that do not include the 
Hilbert Einstein Action $[\underline{8}, \underline{47}]$, (nor any other stringy or area invariance/gravity related variations of an action [ $\underline{45}, \underline{23}]$ ). Applying these principles to EPR entanglement, Path Integrals and propagator operators of virtual and physical (aka real) particles, we introduce a multi-fold universe $U_{M F}$ where non-locality is achieved by locality ${ }^{11}$ : additional paths are activated and available in the Path Integrals to always include paths where entangled particles can at any time instantaneously meet ${ }^{12}$.

$U_{M F}$ goes beyond the pseudo-Riemannian manifold envisaged by Einstein's GR [48] with multiple folds dynamically activated besides the main pseudo-Riemannian manifold, and impacting behaviors on the main manifold by offering new paths to be included in the Path Integrals). The magic is that, doing so, gravity-like effective potentials, and curvatures, appear ${ }^{13}$.

Except for later sections or where mentioned, we discuss our theory in a "universe" $U$ (of class $U_{M F}$ ), that satisfy our proposed "principles", axioms or postulates. We push the implications of our theory for $U$ as far as we can, without assuming or claiming that $U$ be our physical universe $U_{\text {real }}$. Yet, unless said otherwise, we apply today's physics to U as it is defined on $U_{\text {real }}$ and refer to other works or analyses as if also valid for $U$. Until we have validation that $U$ is or has properties of $U_{\text {real }}$, we do not imply that it is. However, in later sections, we discuss some validations and applicability of our model in $U_{\text {real }}$. Even if $U_{M F} \neq U_{\text {real }}$, we believe that modelling $U_{M F}$ implication still provides interesting insights.

\subsection{Outline}

The paper is structured as follows. In section 2, we will review Path Integrals and their relationships to classical and quantum physics. Instead of giving an exhaustive presentation, we will focus solely on recalling what matters for our approach. Section 3 is devoted to introducing and motivating the notions of multi-fold universe $U_{M F}$ and its impact on Path Integrals. In section 4, we will illustrate how the EPR entanglement of real and virtual particles can be handled within a multi-fold universe. It is in this section that we will discover that our ideas and the mechanisms of $U_{M F}$ create gravity-like effective potentials, and effective curvatures; which motivates most of our claims and the rest of the thought process and model. Section 5 will discuss a first set of implications for Quantum Physics; in particular the discovery that the proposed fold mechanism implies a AdS(5) (Anti de Sitter space) tangent dual to the background spacetime of the universe $U_{M F}$, which links our approach to many other works in GR, QFT, superstrings, CFT, quantum gravity. In $U_{M F}$, some of the relationships encountered elsewhere naturally occur. We will also revisit some considerations in non-locality, and discuss spin and torsion. In section 6, we will extend the analysis to other examples of entanglements, including macroscopic situations met, for example, in solid state physics with superconductors, and other quantum materials, where we will predict gravity-like attractive potentials within, and possibly in the immediate surroundings, of the quantum materials. Section 7 will revisit the gravity-like behaviors and implications for properties of quantum gravity while also positioning our approach with respect to other works relevant to gravity. With a solidifying semi-classical model, we will examine in section 8 , the implications of combining gravity with the Standard Model, i.e., $\mathrm{SM}_{\mathrm{G}}$, and in particular show how gravity effects can be non-negligible at the $\mathrm{SM}$ scales, and that the $\mathrm{SM}_{\mathrm{G}}$ can be sufficient to address some open issues around the Standard model, without requiring other New Physics (at least for these aspects). Our discussion of the magnetic monopoles will also be illustrative in terms of the implications for New physics. In section 9, we will use our results so far to reconstruct the spacetime in $U_{M F}$ from the ground up. In this model, spacetime is discrete, fractional and (multi-) fractal, built by random walks and with non-commutative (and possibly non-associative) geometry, which will allow to maintain Lorentz invariance (and Physics covariance) till very small scales. Spacetime and particles consist of microscopic black holes, that among other things allow semi classical approaches till very small scales. We will discuss discrete spacetime and the Standard Model with a solution to the Yang Mills mass gap problem. We will challenge the conventional weak gravitation conjecture, introduce new life cycle options for black holes and discover surprising unification hypotheses of gravity with QCD and Electroweak interactions. Section 10 will bring us a grand finale with input addressing cosmological mysteries around inflation, dark energy, cosmological constant and dark matter; again, without the need of New Physics beyond adding gravity to the Standard Model in $U_{M F}$. Before concluding, in section 11 we will discuss possible validation or falsification of our approach as well as possible applications, including farfetched ones. In our conclusions in sections 12 and 13, we summarize and discuss our findings. Our intent is primarily to communicate with this paper the concepts and illustrate the implications of our model in $U \in$ $U_{M F}$. In the future we will revisit each aspect with more details and rigour. We invite collaboration to these next steps.

\section{PATH INTEGRALS}

Actions, Lagrangian and Hamiltonian mechanics [47] and Richard Feynman's introduction and interpretation of Path Integrals [46] are among the most important formalisms in physics, that it be classical physics including GR or Quantum Physics (Quantum Mechanics, Quantum Field Theories and Gauge Field theories). Indeed, it seems that almost everything in physics can, and should, be expressed through a principle of extremized Action (e.g. the principle of least action derived from the classical Lagrangian or from symmetry considerations [325] in classical physics) or as a path or field integral using an Action for Quantum Physics $[\underline{51}, \underline{52}, \underline{53}, \underline{54}, \underline{55}]$ with possible ordering of operators $[56]$ and/or symmetry considerations [57]).

Historically, Dirac emphasized the importance of Lagrangian [52] in Quantum Physics, which in turn inspired Feynman and others ${ }^{14}$. Feynman proposed Path Integrals as a functional integral used to formulate quantum mechanics 
$[\underline{53}, \underline{46}]$, and to derive the Schrödinger equation from the Path Integral formulation and vice versa. Feynman showed that the function to extremize over different functionals can be formulated as a physical Action, itself expressed as a function of the system Lagrangian when it is known, where the Action or Lagrangian are usually equal to, or derived from the classical Action or Lagrangian. The Path Integral evolution to field integral consists into integrating over fields the corresponding operators $[\underline{57}, \underline{58]}$. The (classical) Action is the phase acquired by quantum evolution between two fixed endpoints. All of quantum mechanics and Quantum Field theory (QFT) can be modelled from the following assumptions: (1) The probability for an event is given by the squared modulus of a complex number called the "probability amplitude". (2) The probability amplitude is given by adding together the contributions of all paths/field in the configuration space. (3) The contribution of a path is proportional to $\left.e^{\left(i \frac{\delta(t)}{\hbar}\right)}\right|_{T}$, where $S(t)$ is the action given by the time integral of the Lagrangian (or density) $\mathcal{L}$ along each path $\mathbf{T}$ (or field density $\mathbf{T}$ on any surface $\Sigma \mathrm{T}$ ).

$$
\left.S(t)\right|_{\mathbf{T}}=\left.\int_{0}^{t} \mathcal{L}\left(q\left(t^{\prime}\right), q\left(t^{\prime}\right), t^{\prime}\right) d q\right|_{\mathbf{T}}
$$

In an upcoming section (See 9.13), we will discuss how the mechanisms of $U_{M F}$ might actually motivate why action extremization (and as a result Path Integrals) actually models all quantum (down to Planck scales), semi classical or classical Physics.

Classical physics is covered by equation (1). This is shown by observing that, at classical scales (i.e., at large $S$ ), the most likely paths are characterized as the one that minimizes the classical action (i.e., the paths where $\delta_{\mathrm{T}} S=0$ are the ones avoiding destructive interferences). This is the well know classical physics principle of Least Action [64].

Today, the evolution of most ${ }^{15}$ physical systems can be modelled as a sum of Lagrangian (densities) covering fields, particles and interactions. Hamiltonian and Path Integral approaches have also been used for constructive quantum field theory $\left[\underline{65}, \underline{66}, \underline{67]}\right.$ to build non-perturbative $\mathbf{Q F T}^{16}$. All this to indicate that constructive quantum field theory is hard but evolving promisingly and able to already construct many fields. It is expected to work as long as the Wightman axioms $[69]^{17}$ are satisfied.

These considerations are relevant to our paper: our objectives include modelling phenomena observed at large scales. Therefore, we do not want to be restricted to a perturbative approach that would restrict the scales in scope. Also we are aware of the gravity renormalization challenges $[43, \underline{44}, \underline{23}, \underline{59}, \underline{60}]$ that make the perturbative approaches diverge. So, we assume that the axioms are satisfied, in spacetime; even with the twists introduced by the multifolds.

Note also that the Path/Field Integral applies as well to nonrelativistic as to relativistic equations [84]. See for example the Klein Gordon equation, and Lagrangian used to recover the propagators of relativistic particle (e.g. massless and massive bosons), or the Dirac equation for fermions $[\underline{73}$, $\underline{74}]^{18}$; albeit they are actually rather quantum field equations because the particles numbers are not constant $[\underline{72}, \underline{16}]$. Computations in potentials also exist $[\underline{75}, \underline{76}]$.

All this is relevant, because we want our approach to encompass relativistic and non-relativistic particles and fields (in flat and curved spacetime). Path Integrals are formalized with the distribution theory [81] and they can be extended and generalized, for example, to topology spaces. This is widely used in constructive theory including the models of LQG with its spin networks $[\underline{25}, \underline{83}]$. This view is also at the core of our multi-fold mechanism. Therefore, we consider that the Path or Field/Functional Integral can be written as:

$$
\begin{gathered}
\mathcal{P} \boldsymbol{I}(\Phi, f)=\frac{\int_{\Gamma} e^{\frac{(i \Phi(\gamma))}{\hbar}} f(\gamma) \mathcal{D}_{\gamma}}{Z(\Phi)} \\
Z(\Phi)=\int_{\Gamma} e^{\frac{(i \Phi(\gamma))}{\hbar}} \mathcal{D}_{\gamma}
\end{gathered}
$$

Where $\Gamma$ denotes a space of paths / fields / geometric objects and $\mathcal{D}_{\gamma}$ is a Lebesgue-type flat measure in a space of paths/fields/geometric objects. It is typically not well defined from a mathematical point of view and cannot be used as a reference measure, but it can be normalized by $Z(\Phi)$ (partition function). $\mathcal{P} I(\Phi, f)$ can be seen as a linear continuous functional on a suitable linear space of test functions $f$, that defines a distribution $T e^{(i \Phi) / \hbar}$ as [81]:

$$
<T e^{\frac{(i \Phi)}{\hbar}} \mid f>\triangleq \mathcal{P} \mathbf{I}(\Phi, f)
$$

Transformations of $\Gamma$ are handled as usual (i.e., changes of variable and Jacobian impact on the measure), and we can see that it can be seen as changing the action $\Phi$ and the distribution on the original $\Gamma$ : the new actions can be seen as transformations, or additions of new geometric objects. Additions of new objects expand the functional integrals in equations (2) and (3). The approach we discussed in this paper is inspired by these considerations. Finally, with $\Phi$ modelling actions or suitable transformations of actions or paths, $\left\|\frac{e^{i \frac{\Phi(\gamma)}{\hbar}} f(\gamma)}{Z(\Phi)}\right\|^{2}$ provides the probability to observe a field or wave function evolving according to the path $\gamma$. The Path Integral, which defines the probability to evolve in a certain way, is often known as the "sum over histories", where each path $\gamma$ between the different states is a history. In our work, we do follow this generalized interpretation, but with a set of what $\gamma$ are allowed in $\Gamma$, where the $\Gamma$ considered are broader than what is currently considered in Quantum Physics theories (in $U_{\text {real }}$ ). However, the set is also differently restricted than what relativistic quantum mechanism and QFT typically allow. At the difference of for example [ $\underline{74}, \underline{339}]$, paths with space like portions (i.e., paths that have portions outside the light cone of a point on the path) are not allowed in $U_{M F}$.

It is worth emphasizing two properties of Path Integrals. Path Integrals respect the uncertainty principle and anticommutativity between position and momentum [247] simply because the paths are random walks where velocities 
standard deviation diverges at any point: paths are not differentiable but rather fractals or of fractional dimensions. We will get back to this. The Path Integral formalism is also covariant (Lorentz covariant) as it amounts to a correlation between two points (i.e., paths starting point and ending point in spacetime). Although QM and QFT are background dependent theories, our approach even in the continuous (quantum, semi classical and classical cases) is background independent, a key feature of QG [25, 249, 248], that is also believed to explain why QFT inspired quantization of $\mathbf{G R}$ are not renormalizable, and explaining why we will have better chances from the onset.

\section{A MULTI-FOLD UNIVERSE WITH NO SUPRA LUMINOUS INTERACTIONS OR PROPAGATIONS}

\subsection{Motivations}

The intuition that motivated our approach, and this paper, is that quantum entanglement and EPR should be explained by the structure of spacetime that implements it, or is impacted by it: it is the responsibility of spacetime to ensure that nonlocality in the background spacetime be supported locally somewhere else, in its structure (i.e., distant spacetime points are collocated elsewhere for some other measure, or criteria). We bet that, doing so, will generate a spacetime with the right macroscopic behavior, built to support such strange requirements. To validate such intuition, we had to propose a universe, with such properties. In fact, as in GR and in most of the different quantum gravity theories, spacetime and gravity are facets of each other, it is also normal to suspect that gravity may appear, if our intuition about entanglement is right. Therefore, and in order to model our intuitive model, we propose a universe where, whenever entanglement takes place (at a common spacetime point), spacetime evolves to ensure the possibility of (instantaneous) exchanges, a priory not communications, between the entangled entities when they become space like; all other considerations being unaffected. That is our theory, model and proposal in the present paper.

Certainly, this does not seem possible in our universe $U_{\text {real }}$ unless if we were willing to accept in the formation of invisible and traversable ${ }^{19}$ wormholes in $U_{\text {real }}$ that would link entangled entities ${ }^{20}$. In addition, even if all this were resolvable, in our opinion, it seems to help little if we establish that wormholes would require large amount of time, even if finite, to be traversed by (exotic) matter). Indeed, it would not match or explain EPR entanglement with its "spooky instantaneous action at distance" that we aim to address. So, instead of models like $\mathbf{E R}=\mathbf{E P R}^{23}$, we assume that exchanges take place outside our 4-D spacetime through a new structure activated by entanglement and linking the entangled entities via enabled / activated allowed paths that also contribute to $\mathcal{P} \boldsymbol{I}(S, \psi)$ : we suggest expanding $\Gamma$, the set of all possible paths, to include such new paths. In addition, our approach makes sense only if we also assume that nothing, absolutely nothing can propagate faster than $c$, the speed of light. Indeed, otherwise, such exchanges are all what it would take. So, no path of $\mathcal{P} \boldsymbol{I}(S, \psi)$ can be traveled faster than $c$ (i.e., no path with space like portions with respects to any other points on the path or said differently, no path venturing outside the light cone for the extremities of the path. This has profound consequences; but some of its implications can be relaxed in good approximations of nature; if we understand the limitations just discussed.

The idea is then to pursue the logic of our reasoning to where it will lead us, rather than just stop at the apparent extraordinary assumptions and implications for spacetime in our universe $U_{\text {real }}$, or just stop at AdS. For these reasons, we work in a hypothetical universe which has the right properties to support our intuition and discuss what happens. We will do this as a logical progression: starting with a spacetime $\mathcal{R}_{B G}$ which, without our new model, would be analogous to $U_{\text {real }}$ spacetime We then add the multi-fold mechanisms needed to support our intuition. This first step describes in good approximations the new phenomena that result from our model at classical, semi-classical and quantum scales. At some point though, we will reach conclusions hinting that other steps need to be taken. We will consider quantization of the multi-fold universe spacetime results from our first analysis. Although an objective, it was not immediately apparent that such a quantization was necessary. For most purposes and at the scale encountered in most of physics, it seems that it does not need to be detailed: the continuous spacetime and multi-folds are good enough approximations just like essentially all of today's physics models. In other words, semi-classic approaches to spacetime/gravity works. We will see that they works for scales smaller than usually assumed. But the consistency of the derived discrete spacetime analysis is also interesting and should inspire others working on such models and foundations of Physics and Quantum Gravity. It is also this discretization that gives us the confidence that no gravity related singularity will appear and that the model will converge and be renormalizable (for this, we also want background independence).

\subsection{Multi-fold universe and Path Integrals}

Let us introduce the notions of multi-fold universe $U_{M F}$ associated to a pseudo-Riemannian manifold $\mathcal{R}_{B G}$. For now, $\mathcal{R}_{B G}$ is what would represent our universe spacetime, with all the properties usually associated to it (i.e., in $U_{\text {real }}$ ) like local curvature (and possibly torsion ${ }^{24}$ ) (described the curvature (and torsion) tensors), a 3+1 dimension, a Minkowski metric, Lorentz symmetries and Covariance-at least locally. So, $U_{M F}$, a multi-fold universe consists of $\mathcal{R}_{B G}$ plus a set of additional manifolds. The folds are defined in the proper time and reference frame following the particle when tagged to a particle and the reference frame of the center of mass of the two particles, when two particles are involved. The difference between both cases will become clear as we progress with the setup of the model. Unless said otherwise, conventions for basic geometry follow the terminology and notations (coordinates, metrics etc.) described in the 
corresponding section at the beginning of [25]: Greek index symbols refer to 0-4 over time and space. Latin indexes go from 1-3 and refer to the space only indexes.

$\left.\mathcal{R}_{F}\left(x^{\mu}\right)\right|_{\boldsymbol{F} \in \boldsymbol{B}\left(x^{\mu}\right)}$ where $\mathcal{R}_{F}\left(x^{\mu}\right)$ can be considered as a new pseudo-Riemannian manifold associated to $x^{\mu}$ for $\mu=$ $\{0,1,2,3\}$ in $\mathcal{R}_{B G}$ and there may a set (i.e. a bundle) of such folds $B\left(x^{\mu}\right)$ that are activated by physical events. $\mathcal{R}_{F}\left(x^{\mu}\right)$ has its own curvature (and torsion-although we will not add any in our folds ${ }^{25}$ ), and, when activated, it becomes available to Path Integrals $\mathcal{P} \boldsymbol{I}(S, \psi)$ for entities encountering a support mapping domain and mapping to it. The notions of encountering and mapping will be clarified as we enumerate the $\mathcal{R}_{F}\left(x^{\mu}\right)$ of interest. We assume that the same laws of physics (in particular, Path Integrals, propagators, Hamiltonians, Lagrangian and Actions) as in $\mathcal{R}_{B G}$ apply within each fold (at least for now), except for interactions per the tenancy model discussed below.

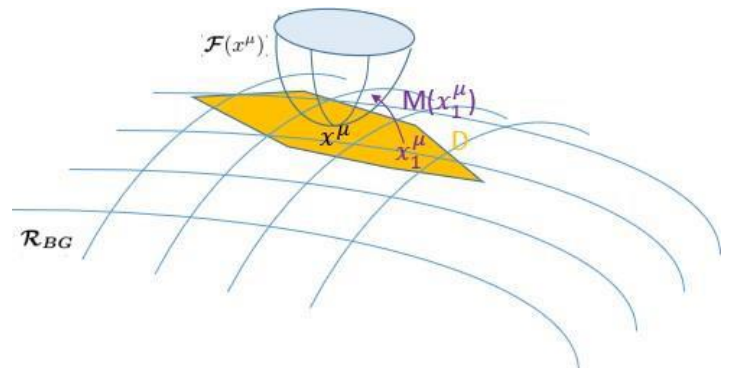

Figure 1: Illustration of a generic fold $\mathcal{R}_{F}\left(x^{\mu}\right)$ activated around a point $x^{\mu}$ with a mapping $M$ that indicates how other points in the support domain $\mathcal{D}$ of $M$ appear on $\mathcal{R}_{F}\left(x^{\mu}\right)$ Paths crossing the support domain $\mathcal{D}$ add a contribution from $M\left(x_{1}^{\mu}\right)$ (an entry point), from where some paths are considered on $\mathcal{R}_{F}\left(x^{\mu}\right)$ (to an exit point).

Plausible paths expand to each activated fold with a mapping:

$$
M\left(F\left(x^{\mu}\right)\right): \mathcal{D}(M) \cap \mathcal{R}_{B G} \rightarrow \mathcal{R}_{F\left(x^{\mu}\right)}
$$

where $\mathcal{D}$ denotes the support domain of $M\left(F\left(x^{\mu}\right)\right)$; which is defined by the fold activation events as discussed after. The mapping, when activated, produces an effect in $\mathcal{R}_{B G}$ through the impact on $\mathcal{P} I(S, \psi)$, associated to the quantum wave function $\psi$, from computing it now on $\Gamma \cup M\left(F\left(x^{\mu}\right)\right)$ for $x^{\mu} \in \mathcal{R}_{B G}$. So, in a multi-fold universe $U_{M F}$, folds are activated for one reason or another by events occurring in the background spacetime $\mathcal{R}_{B G}$. Examples of events will be presented later. When an activation event occurs, paths in the folds that have been activated by the event become plausible in addition to those in the background spacetime $\mathcal{R}_{B G}$.

Waves and fields in a fold $F\left(x^{\mu}\right)$ contribute to the Path Integrals when paths in $\mathcal{R}_{B G}$ encounter $\mathcal{D}\left(M\left(F\left(x^{\mu}\right)\right)\right)$. This is how we propose that the activated folds are "felt" and impacting physics in $\mathcal{R}_{B G}$. The reasoning being that as the folds evolve, the support domain will be defined so as to maintain an analytic behavior along the path of propagation of what caused the event (versus a distribution behavior that is not smooth) of the impact, i.e., mapping, on $\mathcal{R}_{B G}$ while the mapping also enforces that some points on the folds can also be exit points back to $\mathcal{R}_{B G}$. Without the latter, no end point would map to an end point in $\mathcal{R}_{B G}$.

The properties of $\mathcal{R}_{F}\left(x^{\mu}\right)$ and behavior, kinematics or dynamics depend on the events that have activated it. If a bundle of folds $\mathcal{B}_{\text {active }}\left(x^{\mu}\right)$ is activated by an event the set is again defined by the nature of the event. Focusing on Feynman's Path Integral interpretation, when events occur along a path; new paths in newly activated folds associated to the event are also to be considered (e.g. paths in the bundle of folds $\mathcal{B}_{\text {active }}\left(x^{\mu}\right)$ with possible different curvatures from $\left.\mathcal{R}_{B G}\right)$. Activation events are local to what happens locally and the activation initiates where the activation event takes place. We also propose that folds are single tenant with hard partitioning per particle: there are no other particle to interact with the first particle's paths on its fold instance, other than with Higgs bosons, to preserve masses, and as spacetime.

If multiple particles encounter its support domain, each particle is mapped on its own instance of the fold. Interactions however can take place between particles in different instances at the end point of a fold (entry point or exit point), as will be explained later. Intuitively, this is motivated by the fact that observable interaction effects need to take place in $\mathcal{R}_{B G}$.

If many folds are activated forming a bundle, it is not clear how to assume that either a particular fold gets an interaction or why it would randomly take place in all the folds (of the bundle); we would have no real way to propose a computation model to track the different options; the model would not be reasonably tractable. Also, interactions in such folds could create particles out of nowhere ${ }^{26}$ and impact conservation rules in ways that would not make sense in $\mathcal{R}_{B G}$ and certainly, are not observed ${ }^{27}$. Instead, it is more straightforward to assume that interaction if coming from the fold takes place when making it back to $\mathcal{R}_{B G}$.

However, the folds are spacetime also, just curved. Therefore, the tenancy model is to be understood as applying to particles. A uniform field in spacetime and tied to it would also be present and interact in a fold. So, Higgs and the Higgs field are present in the folds and as a result the particle propagator remain associated to their mass in the folds. Similarly, if for example inflation (with many particles random walk responsible for exponential growth as discussed later on) was described with just a same type of particles, the inflation, rather than all the particles encountered today, it would also be present in the folds. It is motivated by the fact that otherwise, particles could mutate in ways not intended by the mechanisms designed to solve EPR paradoxes and no observed in practice.

When deactivation events take place, the fold disappears (i.e., it is no more available) to Path Integrals $\mathcal{P} I(S, \psi)$ for entities in $\mathcal{R}_{B G}$. In such case, their contributions to the Path Integral are stopped from the moment that the support domain of the mapping disappear at a point of a path and no histories are lost in the folds ${ }^{28}$. Also, if folds were reactivated, they would not carry any residue of what happened.

Deactivation is local: the multi-fold impact on the Path Integrals and the associated mappings disappears 
progressively; something that propagates at $c$. It's our hypothesis, it does not really matter. The intuitive motivation is to minimize/avoid violating conservation laws in $\mathcal{R}_{B G}$. As well as to avoid problems similar to the wave function collapse in Quantum Physics.

With our proposal, paths are immediately no more available due to the end of the mapping at the exit point (where what justifies the event to deactivate would take place). The rest can take its course and does it outside $\mathcal{R}_{B G}$, and $U_{M F}$. We don't know the physics and dynamics there and, frankly, we don't really care for our work ${ }^{29}$.

At this stage, we do not expect additional events other than activation $^{30}$ and deactivation of a fold $F\left(x^{\mu}\right)$. Folds, and bundles of folds, have their own dynamics (and kinematics). We do not restrict $U_{M F}$ to having folds whose dynamics would be governed only by say Einstein GR equations (in same spacetime or with additional dimensions, compacted or not). These would just be variations of $U_{M F}$. As such, superstring theory (with AdS/CFT correspondence conjecture and the $\mathbf{E R}=\mathbf{E P R}$ conjecture, already mentioned) are particular variations of our approach. They may not always be able to match all the features of $\mathcal{U}_{M F}$.

By construction, conservations laws are respected in $U_{M F}$ : paths propagating in the activated folds $\mathcal{B}_{\text {active }}\left(x^{\mu}\right)$ are weighted with a probability to entering the fold (think of a small coupling constant) which is expected to be very small. Paths entering from $\mathcal{R}_{B G}$ will "exit" in $\mathcal{R}_{B G}$ at deactivation: nothing is lost, even information ${ }^{31} . T$ symmetry is expected to be violated because of the mechanisms of activation and deactivation of folds (and fold dynamics) which are clearly not reversible: e.g. mappings and paths on the sphere cannot be expected to lead to activation or deactivation as no interaction takes place in a single tenant fold. $\mathrm{T}$ violation usually means irreversibility or being away from equilibrium. Other symmetries are expected to be violated as will be discussed later.

A multi-fold universe $U_{M F}$ can at every spacetime point dynamically become a non-Hausdorff manifold [92]. This is to be related to the proposal [93] that Path Integrals have fundamentally themselves a non-Hausdorff functional structure. It makes sense that the two points of view (nonHausdorff manifolds and non-Hausdorff functional) meet in the formalism of $U_{M F}$. Such structures have been met before in spacetime models ${ }^{32}$, typically when bifurcations occur, or particles can arrive simultaneously at different places (at same proper time) [93]. Yet a clear difference is that our model is not restricted to non-Hausdorff structures occurring only at very smalls scales (within a quantum uncertainty region).

Although our proposal may appear initially physically counter-intuitive, we can argue that it is certainly not much crazier, and possibly with more intuitive dynamics, than the $\mathbf{E R}=\mathbf{E P R}$ conjecture. In fact, once accepted, the multi-fold mechanism clarifies in our opinion aspects of that conjecture, concretizing it in $U_{M F}$ beyond the limits of AdS spaces where that conjecture has been mostly considered.

The consequences of the model should become clearer when we describe events in a multi-fold universe with concrete activation and deactivation events (for EPR entanglement) and how these events activate or deactivate folds.

In an upcoming section, we will present concrete examples of activation and deactivation of bundles of folds $\mathcal{B}_{\text {active }}\left(x^{\mu}\right)$. The list of events of interest may not be complete: other physical phenomena may benefit from our approach. In this paper, we will not investigate or discuss any other phenomena besides entanglement.

\subsection{Absolutely no supra luminosity within $U_{M F}$ and respect of laws of Physics within single tenant (multi-) folds}

Our approach assumes that, within $U_{M F}$, we have absolutely no supra luminous exchanges (i.e., all moves, exchanges or propagation of particle, field, event or interaction are maxed at $c$ ); with only the exception of what falls within the uncertainty principle ${ }^{33}$. It means that at any time, no path can become space like, i.e., no path can venture outside the light cone of any other part of the path. Paths that would do so are not allowed in the Path Integral and can be understood as filtered out by the Path Integral or, said differently, associated to a zero probability/amplitude, or a null dimension fold. As already mentioned, this is fundamentally different from what is usually ${ }^{34}$ done in conventional QFT as discussed for example in $[\underline{74}, \underline{339}]^{35}$. Yet, our prescription does not distinguish based on classical vs. non-classical paths. We agree that the latter must be included in the path integrals.

We also expect respect of the same laws of Physics as in $U_{\text {real }}$ in $\mathcal{R}_{B G}{ }^{36}$, with the tenancy caveats. In each activated fold $\mathcal{R}_{F}\left(x^{\mu}\right) \in \mathcal{B}_{\text {active }}\left(x^{\mu}\right)$, we assume that we only have propagation of particles and fields but no interaction, except with Higgs. The folds are also 2-D in a 3-D space type of spacetime with time index and scales. We provided an intuitive motivation for this already. It can also be motivated by reasoning that folds will very rapidly appear like a variation on the Kaluza-Klein models $[254,256]$ with the activated fold bundles present only at (end) points where they have been activated and with some dynamics ${ }^{37}$, and with details out of scope of our model as they are not expected to affect the approach ${ }^{38}$.

As paths from integral paths cross the support domain of the mappings, they encounter always evolving folds (see after). If interactions were also taking place within the folds, that would imply that we really encounter a Kaluza-Klein spacetime with varying fold radii and that these folds also would play a role in particle interactions and behavior beyond entanglement.

That was simply not our objective and it is not motivated by the entanglement use cases of EPR that we try to address. So, with the hard-partitioned single instance fold tenancy principle, we assume that it is prevented by the mappings. The mappings only create a (multi-)fold instance per entangled entities. And entities with paths that encounter the support domain do not get to share the fold instances and therefore do not interact within each of their activated folds (i.e., Actions, Hamiltonians or Lagrangian drop the interacting components on the folds - these only appear in at 
the end points $)^{39}$. Of course, such concepts could be revisited in future works. They may be worth exploring in the context of superstring theory, for example, where compact, albeit static and global, dimensions are involved. Considering dynamic dimensions, different at each spacetime point, or triggered by events, may lead to interesting results, if it has not yet been explored.

We add that our model is consistent considering the behavior of gravity. In a 2-D spatial universe, as are the folds that we propose in this paper, gravitation does not modify curvature. Therefore, changes of the interactions or the folds based on how many particles/paths encounter the support domain of the mapping do not change the forms, kinematics or dynamics of the multi-folds nor the mappings. It is consistent with 1+1-D GR where normally gravity is only topological without propagating degrees of freedom, and no curvature can be introduced, (but de Sitter can exist with matter) [23, $\underline{250}, \underline{251}]$.

In our case, per the above, we assume that no additional gravitation within a fold exist beyond the effect induced by the spherically symmetric curvature of the folds. And so again, the particles do not interact with others (e.g., gravitons) in the fold, they are not entangled with others nor do they generate virtual particles (no boson exchange, no polarization, no vacuum energy) in the (multi-)folds, except for Higgs boson, and possibly other fields to be determined in the future, filling the spacetime. Again, this is the model of hard multi-tenancy of the folds for the particles, enforced by the mappings and folds, which appear as if an instance is allocated per particle crossing the support domain of the mapping $\mathcal{D}(M)$.

Interactions are only possible at the exit point, and of course at the entry point which is in $\mathcal{R}_{B G}$. Intuitively, the requirement for no supra luminosity is essential for causality and consistency with our motivation. The discretization of the model, motivated later, will in fact illustrate more effectively that this is indeed an absolute requirement, whose violations in some models and calculus must be seen as approximations of reality. In a continuous, classical and semi-classical context, we must add this as a separate requirement ${ }^{40}$. In $U_{M F}$, the results of conventional QFT and Path Integrals with space like paths are only approximations, resulting from these of computation. They usually do not matter because even when allowed, correlations, or spread of propagator outside the light cone are limited and often negligible and as a result it is considered consistent with causality and relativity: no signal can be used for supra luminous communications.

Yet, the approximation impact significantly how vacuum ${ }^{41}$ and how particles can or cannot be modeled in QFT. At the minimum, awareness of the approximations, should remove assumptions that anything would be entangled with anything in QFT or that particles would be meaningless in QFT. We do not agree with these assumptions. As the implications are really at the level of approximations of the model, we believe that, in practice, it can be relaxed for the sake of computations and for the purpose of most modelling: after all, QFT has done very well, so far, without following such a point of view.

\subsection{Constructive axioms and $U_{M F}$}

It seems logical to add the requirements to support the QFT constructive axioms (already discussed and enumerated earlier in section 2) in the folds (besides holding in $\mathcal{R}_{B G}$ ) $[\underline{65}$, $\underline{66}, \underline{68}, \underline{70}, \underline{71}, \underline{69}$ ]; remembering however that no vacuum fluctuations or energy is present in the multi-folds. It is however possible to repeat our analysis in a universe where some of these requirements are relaxed or ignored just as they may for $\mathcal{R}_{B G}$ in $U_{\text {real }}$.

\section{MULTI-FOLD TYPES, KINEMATICS, DYNAMICS, ACTIVATION AND DEACTIVATION EVENTS}

In this section, we discuss the only set of events and mappings of interest in the context of this paper: the ones related to EPR entanglement and subsequent disentanglement in $U_{M F}$. We express no view on the possible existence or not of other types of events associated to other phenomena.

\subsection{Entanglement and EPR}

Considering our initial motivation and intuition, it is logical that we start the discussion with EPR and Entanglement in general. Entanglement in quantum physics, is a key phenomenon that distinguishes quantum Physics from classical Physics, and it captures much of the mysteries of the quantum world. It is also the clearest example where Quantum Physics challenges our intuition and unambiguously tells us something about its deep connection to spacetime (e.g. locality or no locality, Lorentz invariance, or not e.g., faster than light signals or not), reality (is the wave function something real, are quantum states real), and the quantum state space (Are Hilbert spaces and variations and spacetime tied together besides configuration space or phase space representing subspaces, are there hidden variable or not-to be suitably phrased post confirmation of the violation of the Bell inequalities) [467, 466].

All that is without even discussing all the promises of Quantum information Theory, Quantum Cryptography and Quantum Computation built on Quantum entanglement. The formalism of entanglement is discussed in detail for example in [27], along with a mathematical model; in particular, in terms of entanglement entropy also known as von Neumann Entropy.

A simplified introduction can be found in [467]. Considerations and relationships with quantum coherence and correlations (which are different concepts and warrant care when sometimes mixing them together in QFT and statistical model) can be found for example in [252].

Let us now revisit the relevant aspects of the EPR paradox $[\underline{4}, \underline{5}, \underline{95}]$. We use a particle model. We assume a conventional version of the paradox and so the background fold $\mathcal{R}_{B G}$ is flat. Note that it could also be a curved or twisted spacetime without changing much to the explanation of the EPR paradox or the use of Path Integrals [7, 96]). 
In EPR, two quantum particles are produced and emitted in opposite directions (e.g. to preserve momentum) in entanglement. We say that particles or systems are entangled when their quantum state (e.g. opposite polarization for photons or opposite spin direction for electrons-again imposed by conservation considerations) cannot be described independently of the state of the other(s), even when they are separated by a large distance. In this paper we speak of EPR entangled particles when they are such Bell states, i.e., maximally entangled. We are not detailing or reviewing the importance of the Bell inequalities and their experimental violation validations and implications. Some details can be found at $[\underline{466}, \underline{257}, \underline{258}, \underline{5}, \underline{265}]$ as well as some generalization like for example: [ㄷ6, 259, 196].

In a typical setup of an EPR experiment, a system emits two particles of opposite spins or polarizations and we do not know which particle is in what spin or polarization. Bell showed that they are a superposition of both states until one particle is measured [467]. Each particles motion may be described (at least when far enough from each other) by Schrödinger, Klein Gordon or Dirac ${ }^{42}$ or other relativistic or non-relativistic equations of motion, for example derived from the Lagrangian that applies to it [7, 322].

The combined wave function (of the two particles) is similarly described by the Path Integral of the wave functions with creation and destruction operators, which is again the QFT approach. As explained in [5, 467], we know that observing, later, the quantum state of one of the particles, implies immediately that the corresponding quantum state of the other is fully determined. Hence the paradox: how can this happen as the particles have moved far apart, if the particles states were undetermined until the first measurement? Yet that determination is instantaneous after the local measurement of one of the particles ${ }^{43}$.

In the most widely accepted understanding of $U_{\text {real }}$, Bell inequalities demonstrates (or validate) the nonlocality of Quantum Physics in $\mathcal{R}_{B G}$ by showing that Quantum Physics violates them. The resulting Bell Theorem forbids the possibility of "local" hidden-variable theories, i.e. theories which either supplement Quantum Physics with additional variables or new theories: the assumption of a certain kind of locality is a sufficient condition to derive the inequalities, and experiments validated that Quantum Physics violate this inequality $[\underline{5}, \underline{99}]$.

Non-local hidden variables remain possible $[\underline{467}, \underline{466}]$. The EPR and the Bell theorem have been the sources of many controversies on their implications or understanding of measurement experiments. Some have tried to explain non locality in terms of a hidden variable contributed by spacetime and showed that it amounts to adding a "quantum potential" to the particle motions [101, 102, 100, 103]. Others have argued entanglement of the measurement system to disprove non-locality (by satisfying Bells inequalities) but moving the problem to the experimental setup or creating many worlds [104] (à la Path Integral) where different states of experimental system exist in different worlds with only one encountered by observation $[104, \underline{105}]$. It is also worth reading on the complex answer to EPR, as provided by Bohr [107], and the analysis in [106].
Transactional quantum mechanics does not address, in our view, the EPR paradox if measurement occurrence is not predictable, i.e., we do not know that a measurement will take place and which measurement it will be (and of what): nonlocality is still needed.

So, in all these cases, there is still a need for suitable way to convincingly explain EPR, unless of course if we just want to shut up and compute without wondering, as suggested by the Copenhagen interpretation. Let us note the analysis [108], that uses the sum of histories with Path Integrals to reproduce the Bell inequalities results and with non-locality captured in all the past histories. Using Path Integral to discuss EPR has been done before in $U_{\text {real }}$.

However, we have forbidden supra luminous paths in our multi-fold universe $U_{M F}$. With no supra luminous interactions, the analysis of $[\underline{108}, \underline{261}, \underline{340}]$ is only valid if all considered paths are associated to speed lower than $\mathrm{c}$. This restriction prevents using the model to explain EPR entanglement in general when the entangled particles have moved far away from each other.

Considering the above, the experimental corroboration of non-locality and violation by QM of Bell's inequalities and the examples like the successful quantum teleportation in $U_{\text {real }}[\underline{109}, \underline{110}, \underline{466}]$, we accept that quantum Physics indeed appears non-local in $\mathcal{R}_{B G}$.

This paper provides mechanisms to allow EPR entanglement and QM nonlocality without the paradoxes, and without supra luminosity. The onset of the EPR entanglement is considered to be a triggering event at $x_{0}^{\mu}$. We propose that corresponding folds $F\left(f \text { or: } x_{0}^{\mu}, t\right)^{44}$ are activated so that if measurements take place at $x_{f}^{\mu}$ on one of the two EPR entangled particles, the mapped path for the two EPR entangled particles $\mathrm{can}^{45}$ meet at the antipode of the mapping of $x_{f}^{\mu}: y_{f}^{v}$.

Figure 2 illustrates a possible $F\left(x_{0}^{\mu}\right)^{46}$ as the surface (i.e., a 2-D space) of a 3-D (Spatial) sphere, outside spacetime and tangential to the momentum axis of the particles. At any time $t \leq f$, the mapping $M\left(x_{t}^{\mu}\right)$ to the sphere maps the segment $\left[x_{0}^{\mu}, x_{t}^{\mu}\right]$ to the equator going from $x_{0}^{\mu}=y_{0}^{v}$ to $y_{0}^{v}=M\left(x_{t}^{\mu}\right)$ so that the motion of the entangled particle can support a grand circle of the sphere of same perimeters as the distance between the particles as they move away. With the hardpartitioned tenancy of one instance per particle, interaction between the two entangled particles is allowed only at $y_{t}^{v}=$ $M\left(x_{t}^{\mu}\right)$. As time passes, the folds evolve, and the sphere radius grows as a function of the momentum and time. It also evolves with the center of mass of the two EPR entangled particles that it tracks ${ }^{47}$. Note that figure 3 illustrates a center of mass that is not moving as we are in its referential. So $x_{0}^{\mu}=x_{C M}^{\mu}$. The intuitive smoothness of the mapping mentioned before, occurs in the spacetime region between the particles. Smoothness for paths encountering the support domain of the mapping will be discussed below with different possible mapping support domains. The shape, kinematics and dynamics of the proposed folds results directly from the symmetries of the folds as well as the need to ensure that the fold mechanisms ensure that paths meet at 
$y_{f}^{v}$, at all time, but not that they become the source of additional new and non-observed physics due to creation or annihilation of particles in the folds with curvature itself changing with time, or disappearance of conserved quantities in the folds.

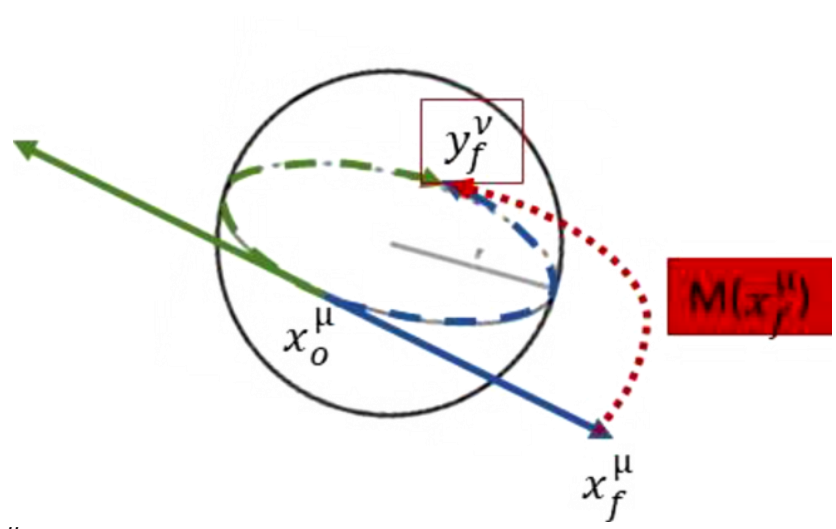

Figure 2: It illustrates a fold $F\left(\right.$ for: $\left.x_{0}^{\mu}, f\right)$ at time $f$ for two EPR entangled particles. The mapping is also illustrated for the segment $\left[x_{0}^{\mu}, x_{f}^{\mu}\right]$ to the equator going from $x_{0}^{\mu}=x_{f}^{v}$ to $y_{f}^{v}=M\left(x_{f}^{\mu}\right)$, for the closest particle (part1). A P-symmetric (i.e., a reflection) mapping exists for the symmetric segments associated to the other particle part2. Any entity meeting $\left[x_{0}^{\mu}, x_{f}^{\mu}\right.$ (part 1$\left.)\right] \cup\left[x_{0}^{\mu}, x_{f}^{\mu}(\right.$ part 2$\left.)\right]$ encounters the support domain of the mapping $\mathcal{D}(M)$ (the figure is for $\mathcal{D}_{\text {tear }}(M)$. This is one possible fold associated to $x_{0}^{\mu}(f)$. As the time, here noted $f$, changes, the fold also evolves to continue to match this figure: the fold grows proportionally to $x_{f}^{\mu}$ itself proportional to the elapsed time (if we assume constant momentum for each particle). With hard-partitioned fold instances per particle, interactions between the two entangled particles are allowed only at $y_{T}^{v}=M\left(x_{t}^{\mu}\right)$, besides the entry point. The fold grows as the particles move apart.

At any time before observation of one of the entangled quantum states, the Path Integral is therefore a sum of the Path Integral in the main background spacetime ${ }^{48}$ plus the Path Integrals on the surface of all the different ${ }^{49}$ possible spheres appropriately sized (i.e., to reflect the probability to take paths on any folds in $\mathcal{B}\left(x^{\mu}\right)$ versus on $\left.\mathcal{R}_{B G}\right)$.

With this construction, the two EPR entangled particles can always have allowed (and activated) paths that ensure that at $t$, a path can have $y_{t}^{v}\left(\operatorname{part}_{1}\right)=y_{t}^{v}\left(\operatorname{part}_{2}\right)$; therefore, allowing the wavefunction to communicate through their extensions in $F\left(\right.$ for $\left.: x_{0}^{\mu}, t\right){ }^{50}$.

Indeed, interaction between particles in the single tenant folds are allowed at that point. This explains how non- locality à la "Bell" occurs in EPR without requiring supra luminous communications, beyond what can reasonably be associated to the uncertainty principle. This mechanism achieves our principles and objectives. We postulate that this is what happened in $U_{M F}$ when two particles are EPR entangled and we will investigate the consequences.

Let us now compute the contributions of $F\left(\right.$ for: $\left.x_{0}^{\mu}, t\right)$ to the Path Integral $\mathcal{P} \boldsymbol{I}(S, \psi)$ for a particle at $x_{t}^{\mu}$. At time $\mathrm{t}$, it is provided by the propagation of a particle with a relativistic or non-relativistic particle on the surface of a 3-D sphere with radius $r$, which is proportional by construction (the mapping) to the (spatial) distance between $x_{0}^{\mu}(t)$ and $x_{f}^{\mu}(t)^{51}$.

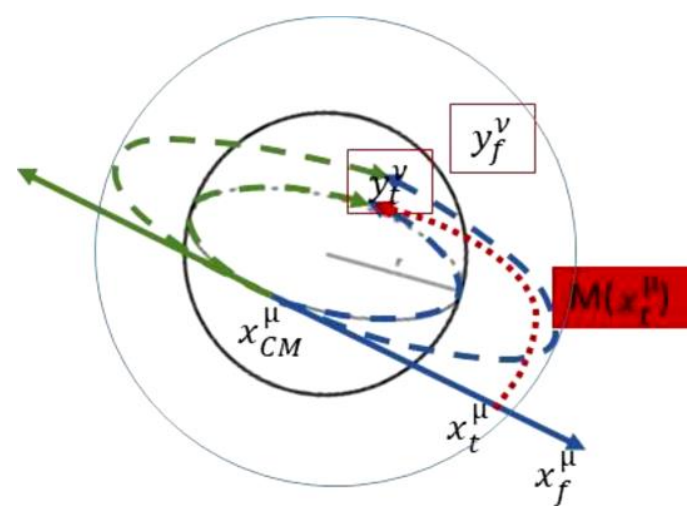

Figure 3: The contribution of $\left.\mathcal{P I}_{\mathcal{B}_{\text {active }}\left(\text { for: } x_{C M}^{\mu}, t\right)}(\boldsymbol{S}, \boldsymbol{\psi})\right|_{(\boldsymbol{p a r t}(\boldsymbol{t}) \in \mathcal{D}(\boldsymbol{M})}$ includes integration over $r$. Here, we assume: $\mathcal{D}(M)=\left[x_{C M(t)}^{\mu}, x_{t}^{\mu}\left(\right.\right.$ part $\left.\left._{1}\right)\right] \cup\left[x_{C M(t)}^{\mu}, x_{t}^{\mu}\left(\right.\right.$ part $\left.\left._{2}\right)\right]$. 
As discussed in section 10 of [77], and in [111], the contribution between $y_{0}^{v}(t)$ and $y_{f}^{v}(t)$ is in:

$$
\begin{gathered}
\operatorname{Int}(t)=\left[x_{C M(t)}^{\mu}, x_{t}^{\mu}\left(\text { part }_{1}\right)\right] \cup\left[x_{C M(t)}^{\mu}, x_{t}^{\mu}\left(\text { part }_{2}\right)\right] \\
\left.\mathcal{P I}_{\boldsymbol{F}\left(\text { for }: x_{C M}^{\mu}, t\right)}(\mathcal{S}, \psi)\right|_{\text {part }(t)_{\in I n t}} \propto \frac{1}{r^{2}} \\
\propto R \\
\propto m_{\text {part }(t) \in \operatorname{Int}(t)} \\
\propto \kappa_{E P R}\left(\boldsymbol{F}\left(\text { for: }: x_{0}^{\mu}, t\right), \operatorname{part}(t)_{\in \operatorname{Int}(t)}\right)
\end{gathered}
$$

CM stands for center of mass between the two EPR entangled particles. Equation (8) shows that the contribution is proportional to the Ricci Curvature Scalar $R$ of the sphere $)^{52}$. These results hold for Euclidian or Minkowski metrics. We are only interested in the proportionality (and not the exact value) as we do not model in this work how contributions from activated folds are weighted versus the paths in $\mathcal{R}_{B G}$. Computations only involve the propagation Action; no interaction terms as already discussed. In (9), $m_{\text {part }}$ designate the mass (or energy converted to mass) of the particle crossing at $\mathrm{t}$ the domain support $D(M)$ of the mapping.

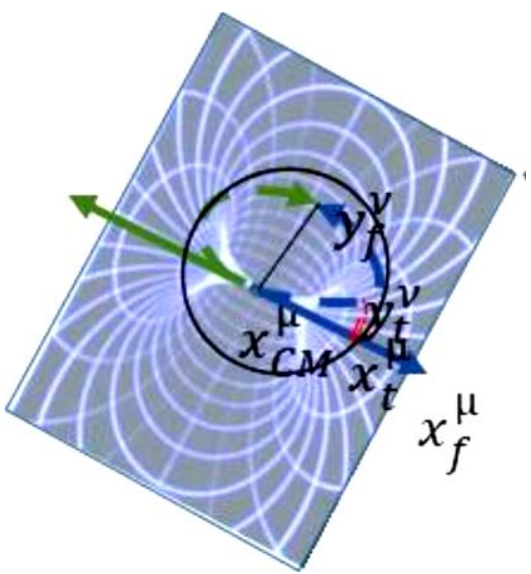

Figure 4: The contribution of $\left.\mathcal{P} \boldsymbol{I}_{\mathcal{B}_{\text {active }}\left(\text { for: } x_{C M}^{\mu}, t\right)}(S, \psi)\right|_{(p a r t(t) \in \mathcal{D}(M))}$ includes integration over $2 \pi$. The bundles of fold are sets of tori. Here, we assume: $\mathcal{D}(M)=\left[x_{C M(t)}^{\mu}, x_{t}^{\mu}\left(\right.\right.$ part $\left.\left._{1}\right)\right] \cup\left[x_{C M(t)}^{\mu}, x_{t}^{\mu}\left(\operatorname{part}_{2}\right)\right]$.

Here $\kappa_{E P R}\left(\boldsymbol{F}\left(\right.\right.$ for $\left.\left.: x_{0}^{\mu}, t\right), \operatorname{part}(t)_{\in I n t}\right)$ represents the weight that a path of $\operatorname{part}(t) \in\left[x_{C M(t)}^{\mu}, x_{t}^{\mu}\left(\operatorname{part}_{1}\right)\right] \cup$ $\left[x_{C M(t)}^{\mu}, x_{t}^{\mu}\left(\operatorname{part}_{2}\right)\right]$ in $F\left(\right.$ for: $\left.x_{0}^{\mu}, t\right)$ carries versus a path in $\mathcal{R}_{B G}$ which is weighted by $\kappa_{\mathcal{R}_{E P R}(B G)} \kappa_{\mathcal{R}_{E P R}(B G)}$. Physically, it can be viewed as the probability associated to having a contributing path from an activated fold associated to EPR entanglement. At this stage, we do not have ways to really quantify $\mathcal{K}_{E P R}(F)$. We assume for the rest of the paper that these coupling constants are constant in $\mathcal{R}_{B G}$ for all entangled particles and folds: $\mathcal{K}_{E P R}(F)$ and $\mathcal{K}_{E P R}\left(\mathcal{R}_{B G}\right)$.

$\mathcal{K}_{\text {EPR }}(F)$ may also depend on a measure of the degree of entanglement (e.g. pure state entanglement vs. partial entanglement) [27].

Such analysis is for future works, but we provide some ideas in section 4.2. For now, we assume only pure state entanglement. Note that we have considered that the support domain of $M$ for each fold is:

$$
\mathcal{D}(M)=\mathcal{D}_{\text {tear }}(M)=\operatorname{Int}(t)
$$

This is an arbitrary proposal: without experimental guidance or a more detailed mathematical formalism, there is no way to decide at this stage. So, we just need a stake in the ground. To smooth a bit along the path of particles encountering the support domain we can rely on the uncertainty principle: we know that in this case (11) will be a wider region around the support domain $\mathcal{D}(M)$ defined in the equation (11):

$$
\begin{aligned}
& \mathcal{D}(M)=\mathcal{D}_{\hbar+\text { tear }}(M)= \\
& {\left[x_{C M(t)}^{\mu}, x_{t}^{\mu}\left(\text { part }_{1}\right)\right]^{-\hbar} \cup\left[x_{C M(t)}^{\mu}, x_{t}^{\mu}\left(\text { part }_{2}\right)\right]^{-\hbar}}
\end{aligned}
$$

In these equations, $\sim \hbar$ designates the fuzziness that results from the uncertain principles and gives width and smoothness. It is also possible that the mapping extends beyond these regions (e.g. isotropic disk of radius r):

$$
\mathcal{D}_{[\hbar]+\text { disk }}(M)=\mathcal{D}_{[\hbar]+\text { disk }}^{(1)}(M) \cup \mathcal{D}_{[\hbar]+\text { disk }}^{(2)}(M)
$$

where:

$$
\begin{aligned}
\mathcal{D}_{[\hbar]+\operatorname{disk}}^{(1)}(M)= & \operatorname{disk}\left(x_{C M(t)}^{\mu},\right. \\
& \left.d\left(x_{C M(t)}^{\mu}, x_{t}^{\mu}\left(\operatorname{part}_{1}\right)\right)\right)^{[-\hbar]} \\
\mathcal{D}_{[\hbar]+\operatorname{disk}}^{(2)}(M)= & \operatorname{disk}\left(x_{C M(t)}^{\mu},\right. \\
& \left.d\left(x_{C M(t)}^{\mu}, x_{t}^{\mu}\left(\operatorname{part}_{2}\right)\right)\right)^{[-\hbar]}
\end{aligned}
$$


In these equations, [] designates options. The support domain could also just be:

$$
\begin{gathered}
\mathcal{D}(M)=\delta\left(x^{\mu}-x_{\text {part }_{1}}^{\mu}\right)+\delta\left(x^{\mu}-x_{\text {part }_{2}}^{\mu}\right) \\
\mathcal{D}_{[\hbar]+\delta}(M)=\left(\delta\left(x^{\mu}-x_{\text {part }_{1}}^{\mu}\right)+\delta\left(x^{\mu}-x_{\text {part }_{2}}^{\mu}\right)\right)^{[-\hbar]}
\end{gathered}
$$

The sphere as a fold in figure 2, is just one among many possible spheres. The bundle of activated folds $\mathcal{B}_{\text {active }}\left(x^{\mu}\right)$ includes all the spheres possible of radius $r^{\prime} \leq r$; a set of torus of small radius $r^{\prime} \leq r$, centered on the axis of the momentum of the two EPR entangled particles. It is shown in figure 3 . As a result, we have several symmetries; the most important one from the point of a fold is the symmetry by rotation by $180^{\circ}$ for traditional EPR pairs. This means a fundamental "spin-2" type of symmetry ${ }^{53}$. The contributions of $\mathcal{B}_{\text {active }}\left(x_{C M}^{\mu}\right)$ to Path Integrals computed for all mapped spheres is therefore in:

$$
\begin{aligned}
& \mathcal{D}_{\alpha}(t)=\mathcal{D}(M)\left(x_{C M(t)}^{\mu}, x_{t}^{\mu}\left(\text { part }_{1}\right), x_{t}^{\mu}\left(\text { part }_{2}\right)\right) \\
& \left.\mathcal{P}_{\mathcal{B}_{\text {active }}\left(\text { for: } x_{C M}^{\mu}, t\right)}(S, \psi)\right|_{\text {part }(t) \in \mathcal{D}_{\alpha}(t)} \propto\left(\frac{1}{r}\right)
\end{aligned}
$$

(by integrating the previous result over $r$ and the $2 \pi$ azimuth angle). The result can also be seen as:

$$
\left.\mathcal{P} \boldsymbol{I}_{\mathcal{B}_{\text {active }}\left(\text { for: } x_{C M}^{\mu}, t\right)}(S, \psi)\right|_{\text {part }(t) \in \mathcal{D}_{\alpha}(t)} \propto \sqrt{|R|}
$$

This is the result of integrating all the Ricci scalar curvature; which are indeed additive per $[\underline{363}, \underline{364}]$; something that we will exploit in section 4.8 .

Per the properties of the multi-fold mechanisms, particles stay on a fold; they do not jump from folds to other activated folds in $U_{M F}$ ), but follows the evolution (growth) with time within the same fold (no interaction is allowed that would support jumps). If it were not the case, we would no more be on folds with spherical symmetric spacetime of dimension $D=2$, and, as a result, folds could create new particles [171]; something that does not match observation of EPR entanglement, nor address the purpose of the folds. We already know that on a 2-D surface gravity is purely topological without additional degrees of freedom of modifying the curvature and no interaction other than possibly at the entry and exit points. This is the reasoning that we mentioned earlier and that explains why we selected 2-D sphere surfaces for the form of the folds.
The entities affected by these phenomena are those that cross points on the axis between the two EPR entangled particles. The effect propagates relative to the center of mass at the speed of each EPR entangled particle. As computed in [77, 112], the effect amounts to introduce an anisotropic effective potential in the direction of $x_{C M}^{\mu}$ :

$$
V_{\text {eff }} \propto \frac{1}{r}
$$

The same reasoning is true for non-relativistic and relativistic particles and computing the Path Integrals with Euclidean or Minkowski metrics [77, 112, 113]. Indeed, the apparition of a potential also appears in Klein Gordon (field) equation for Boson in a curved space (see chapter 5 in [345]) and in Dirac's equations for Fermions (see equation 5.23 in [345] is also always satisfied by spinors when taking the second order version of Dirac's equation which is of the form of Klein Gordon equation).

Again, the effective potential is proportional to the Ricci scalar of the sphere (for a fold) or to the square root of it after integration over all the involved folds and it is attractive towards the center of mass as the curvature of each sphere increases the potential on the sphere in ways that favor not moving away from $x_{C M}^{\mu}$ [346], further shows that are no differences of behavior / propagation between Bosons and Fermion in a 3D sphere (only the levels of energy differ due to the different spin statistics).

Other choices of $\mathcal{D}(M)\left(x_{C M(t)}^{\mu}, x_{t}^{\mu}\left(\operatorname{part}_{1}\right), x_{t}^{\mu}\left(\right.\right.$ part $\left.\left._{2}\right)\right)$ lead to different $V_{\text {eff }}$.

In fact, $D_{[\hbar]+\delta}(M)$ keeps a $V_{e f f} \propto \frac{1}{r^{2}}$. In all cases, in our multi-fold universe $U_{M F}, \mathbf{E P R}$ entanglement means that an emerging effective potential $V_{e f f}$ is felt by entities with a path in $\mathcal{D}(M)\left(x_{C M(t)}^{\mu}, x_{t}^{\mu}\left(\right.\right.$ part $\left._{1}\right), x_{t}^{\mu}\left(\right.$ part $\left.\left._{2}\right)\right)$.

It propagates a wave affecting $x_{t}^{\mu}$ at distances smaller or equal to the distance between $\left.x_{t}^{\mu}\right|_{\text {part }_{1} \wedge \text { part }_{2}}$ from $x_{C M}^{\mu}$, and always smaller that $c t$. Indeed, the spheres grow in radius at speed smaller than $c$ : they grow at the speed of the EPR entangled particles (with respect to their center of mass): the multi-fold effects are massive waves (unless if the entangled particles are massless and propagate at $\mathrm{c}$, in which case the multi-fold effects are massless).

For $\mathcal{D}_{\text {tear }}(M)\left(x_{C M(t)}^{\mu}, x_{t}^{\mu}\left(\operatorname{part}_{1}\right), x_{t}^{\mu}\left(\right.\right.$ part $\left.\left._{2}\right)\right)$, a gravitylike potential appears in between the EPR entangled particles and attractive towards their center of mass. For $\mathcal{D}_{\delta}(M)\left(x_{C M(t)}^{\mu}, x_{t}^{\mu}\left(\operatorname{part}_{1}\right), x_{t}^{\mu}\left(\operatorname{part}_{2}\right)\right)$, it is simply an attractive shock wave in $\left(1 / r^{2}\right)$. The way that the folds follow the center of mass between the two particles may appear surprising: why and how would that happen? It turns out that analyses of EPR entanglement in phase spaces [246] show that EPR entanglement results into extra Wigner function correlations exactly around the center of mass of the two particles: EPR entanglement is a process that involves the center of mass of the entangled particles and there is a deeper relationship between Hilbert space/state space, configuration space and phase space. 
When measurement or disentanglement takes place, the folds in $B_{\text {activ }}\left(x_{C M}^{\mu}\right)$ are deactivated. It is a deactivation event. It can be seen as if the folds "detach" from a state of being tangent to $x_{C M}^{\mu}$ and the mappings $M$ are torn apart as a result. For $\mathcal{D}_{[\hbar]+\delta}(M)\left(x_{C M(t)}^{\mu}, x_{t}^{\mu}\left(\right.\right.$ part $\left._{1}\right), x_{t}^{\mu}\left(\right.$ part $\left.\left._{2}\right)\right)$, it just ends being available to paths.

For $\mathcal{D}_{[\hbar]+\text { tear }}(M)\left(x_{C M(t)}^{\mu}, x_{t}^{\mu}\left(\operatorname{part}_{1}\right), x_{t}^{\mu}\left(\operatorname{part}_{2}\right)\right)$ a wave propagates back to $x_{C M(f)}^{\mu}$ as the tear of the mapping disappears.

As it is a spacetime change, connected to $\mathcal{R}_{B G}$, it seems logical to speculate that it propagates at $c$. In any case we avoid the paradoxes of wavefunction collapse with such a fold deactivation mechanism. For an interesting discussion of the relationship between disentanglement and wave function collapse, as well as looking at disentanglement as spontaneous symmetry breaking, see [490]. As fold kinematics and dynamics (and support domains), especially tear down, are pure speculation, it is hard to say more.

But it seems logical that an entity meeting $\mathcal{D}(M)\left(x_{C M(f)}^{\mu}, x_{f}^{\mu}\left(\operatorname{part}_{1}\right), x_{f}^{\mu}\left(\right.\right.$ part $\left.\left._{2}\right)\right)$ would still feel an attractive potential towards $x_{C M(f)}^{\mu}$, until the mapping to the fold is deactivated at that point.

In all cases, the fold deactivation seems to indicate an irreversible process or, at least, away from equilibrium: it is not $\mathrm{T}$ symmetric. In $U_{M F}$, disentanglement appears as an irreversible process that violate $\mathrm{T}$ symmetry. At this stage, we cannot yet comment on other symmetry violations. We will add considerations throughout the paper as our model description and its analysis evolves. In our view, the fold activation and dynamics are probably also irreversible.

In $U_{M F}$, fold activation is the enabler of entanglement and its manifestation through the attachment of the folds to the entangled particles implemented by the mapping. The presence of multi-folds implements entanglement. Their deactivation coincides with its termination. So, while entanglement is not observable [87], its impact via $V_{\text {eff }}$ (or curvature contributions) is observable and a sign and measure of entanglement. Multi-Folds exist outside $\mathcal{R}_{B G}$ and we cannot observe them either; but again, we measure their effect on $\mathcal{R}_{B G}$ via $V_{\text {eff }}$ or the impact on an effective curvature. Conservation laws and unitarity are preserved for $\mathcal{D}_{\text {h+tear }}(M)$ mappings: a path from any entity from any entity crossing $\mathcal{D}(M)$ can return to the entry point and let it be an exit point. Any infinitesimal wave function contribution can exit. The same argument exists for most other mappings: at any time after deactivation: the mapping points from any entity on $(M)$ onto the fold can be met by paths on the fold that can be used to exit. Whatever is the process of deactivation, all conservations and unitarity can be maintained. Of course, in a model where mappings would not behave this way (variations on our proposal for a multifold universe $U_{M F}$ ), then it could introduce and explain conservation or unitarity ${ }^{54}$ violations. In the rest of this paper, we assume a model with $\mathcal{D}_{\hbar+\text { tear }}(M)$ mappings (unless when discussing explicitly). This is for consistency with the virtual particle events discussed in section 4.4 and after.

\subsection{Other Entanglements}

Similarly, entangled particles may be further entangled with other particles, within the limits of the principle of monogamy (/polygamy for multi-partite entanglement) $[115$, 468]. When no pure states are involved, we need to revert to density operators [27] that define separable and not separable subsystems of the matrix. Non-separable systems are considered entangled and within these systems, we expect to find similar entanglement behaviors (weighted by the density operator) as the attractive potential between EPR entangled particles. We also expect that the attractive effective potential will now be also proportional to a function of the entanglement entropy of the non-separable systems. The function should be such that at maximum entropy (i.e., pure state or EPR entanglement), we recover the $V_{\text {eff }}$ discussed in the previous section. Our model is (at least for now) only valid for systems entangled via local interaction. Otherwise, the considerations of entanglement hierarchy discussed below apply. Indeed, the fundamental mechanism proposed in $U_{M F}$ requires a common entry point to the folds ${ }^{55}$. Entangled particles may also result from other phenomena than entangled emissions. Examples are entanglement through a field or via quantum optics (e.g. entangled polarization and trapped ions) or interactions between particles or with other entangled particles like in the IBM quantum teleportation case $[\underline{109}, \underline{116}]$. The framework proposed here distinguishes EPR entanglement from other quantum correlation. We assume that to achieve EPR entanglement, we start with particles that are neighbors (overlapping particle locations in the wave function) in order to have entanglement-based fold activation events. It amounts, or at least relates, to the locality principle of axiom of QFT. There are however other situations where a system can get entangled with another through some other systems becoming entangled with each other first. Examples are described in $[117, \underline{118}]$. For example, the setup in [118] involves multiple levels of system entanglement: photons emitted by trapped ions in distant ion traps can be entangled by quantum optics (e.g. polarization filters), which in turn entangle the distant ions (sources of the photons) and can be used again to quantum teleport the state of the ions from one trap to the other. This is what we consider to be a hierarchy of entanglements. Accordingly, folds are activated between each ion and its emitted photons. When the photons are entangled through the optical system, folds appear between the photons and between each ion and both entangled photons. As a result, the two ions are hierarchically entangled, but no fold appear between them. Indeed, the ions were never "locally close" at entanglement.

This is another rule of activation of folds in our multi-fold universe $U_{M F}$ : hierarchical entanglements between entities, not local when the entanglement was initiated, is not associated to the activation of folds ${ }^{56}$, at least for now.

However, as for the setup in $[117, \underline{118}]$, attractive forces appear on the entangled entities (through the forces between 
entangled "first order" entities, i.e. non-hierarchically entangled).

In $[117,118]$, the photons are guided in single mode optical fibers. It affects the path of the photons. It does not affect the behavior of the activated bundles other than the spheres, that it contains, matching the movement of the photons in the fibers. The folds are spacetime curvature and not blocked or interfered with by objects in $\mathcal{R}_{B G}$; there is no notion of gravity shield in $U_{M F}$. The mapping between the positions of the particles in $\mathcal{R}_{B G}$ to the folds is key to ensure that paths in each fold can carry back the measurement event that disentangle the different systems.

One can see that the situation can rapidly become complicated as the number of bodies increases; but consistently, each combinations of pairs that other than the spheres, that it contains, matching the movement entanglement/entanglement monogamy principle) create attractive effective potentials and mappings. Hierarchical entanglements do not generate (necessarily) such potentials among the higher order pairs. Multiple entry points to the fold would have to be considered to support them, hence the hierarchical entanglement principle in this paper. The resulting folds with three or more entities depends on the history of production of the entanglement: the resulting activated folds are not necessarily associative. This is an important observation that we will reuse later. It certainly begs to question how large set entanglements (e.g. bulk volume or surface) between space like locations should be treated (e.g. as discussed for example in [14] and in QFT $[\underline{125}, \underline{126}]^{57}$ ). It has also been proposed that entanglement can be also temporal as in [119]. There, we have higher order entanglements, and, per our model, the experiment does not create incompatibilities as no attractive effective potential needs to appear between the entangled photon in the past and photon at the end. Of course, all setups each warrants dedicated case by case analyses.

\subsection{Macro entanglement and generic considerations}

We will discuss examples later in the paper, including superconductors, Bose Einstein condensates BEC which are macroscopically entangled [120] and quantum computing. They are direct applications of what we have discussed so far. However, in each case, it will be worth considering if entanglement is direct, hierarchical or a combination of those. Examples where for examples a particle would exchange another one (e.g. photon or phonon) with another particle so that they get entangled would a priori be hierarchical. But if the entanglement is the product of continuous back and forth exchanges of particles, for all purpose the effect will appear as if between the resulting entangled particles. That being said, force compositions, in hierarchical situations, also result into similar effects that could be observed.

\subsection{Virtual particles, entanglement and no supra luminous propagators}

Path Integrals applied to quantum field theory (or Path Integrals applied to the Klein Gordon, Dirac and other fields Lagrangian or actions) allow computation of the (time ordered) propagator of the associated particles. See for example $[\underline{74}, \underline{339}, \underline{55}, \underline{121}, \underline{122}]$. The probability of realization (observation) of resulting wave functions, or fields, at a given $x_{t}^{\mu}$ can be derived $[\underline{74}, \underline{122}]$, and estimated via scattering computations that lead to the Feynman diagrams and associated Feynman rules. Typically, especially when not considering the no supra luminous paths principle, the relativistic-particle conventional propagator resembles certain curved-space propagators with a wider spread than non-relativistic propagator [ $[\underline{51}, \underline{123}]$.

In QFT, the propagators (expressed in the $x^{\mu}$ domain or in the Fourier domain, i.e., conjugate momentum space); can be non-zero between space like regions, because the conventional Path Integral allows paths outside the light cone of other points on the path. As a result, the wave amplitude can be non-zero outside the cone of light (spacelike coordinates), as are correlation functions between space like regions in $[125, \underline{126}]$. This can also be seen when computing the observed amplitude outside the cone of light for solution of Klein Gordon and Dirac equations [74]. In general, only photons and massless particles stick explicitly with the light cone [124]. In QFT, these challenges are addressed with explanations that the wave functions of propagating particles are almost zero at space like positions significantly away from the cone of light and that observation probability is essentially zero. Therefore, one assumes that there is no contradiction with $c$ as upper limit for "information propagation" or "signal exchanges" $[\underline{125}$, 123]. [126] summarizes even more fundamental problems with relativistic propagator of particles versus fields.

In $U_{M F}$, such examples are problematic and the usually provided explanations mentioned above are not good enough (even if powerful tools giving extraordinarily precise estimations): space like paths are not allowed in Path Integrals. Indeed, their allowances would negate the reason that we needed to introduce the multi-fold mechanisms to address the EPR paradox. So, we assume that conventional quantum mechanics and field computations are only approximations of reality (in this case Path Integrals and propagators) for the ease of computation. The extra filtering steps are painful, they do not lead often to exact expressions. They often may not be worth the effort, in terms of experimental/numerical results. But theoretically, it matters a lot, for the consistency of our approach. Let us see how we can handle physical (real) propagators in our multi-fold universe $U_{M F}$.

Assume a physical particle and its associated propagator and Path Integrals. They are accounting for generations of many virtual particles that may interact with it, with other particles or generate new particles as captured with the Feynman diagrams. In $U_{M F}$, components of the propagators in the momentum space and Path Integrals are filtered to drop out components outside the light cone (i.e., of momentum supra 
luminous $)^{58}$ : particles reaching the light cone must stay on it, interact or disappear. These changes to the Path Integral can be designated as $\mathcal{P} \boldsymbol{I}_{S F}$; which from now on is what is assumed to be meant in all the presented entities or formulas in $U_{M F}$. Every particle or energy entity (i.e., bump) is surrounded with virtual particles that it helps temporarily create and propagate (from the vacuum), relying on the uncertainty principle for their allowance ${ }^{59}$. Entry points of view on the vacuum and virtual particles are presented in $[\underline{264}, \underline{2}, \underline{130}]$. The implications can be modelled as follows with an approach analogous to the EPR multi-folds mechanisms:

- If a particle is located at $x_{0}^{\mu}$, a bundle of folds $B_{\text {activ }}$ is activated as tori of 3-D spheres with as radius up to the light cone radius (organized as cones along the time axis) to handle entanglement of the virtual particles (and antiparticles) that it helps generate.

- $\quad S F$-Path Integrals include normal Path Integrals in $\mathcal{R}_{B G}$ plus sums of Path Integral over the spheres for path within light cone: Virtual particles slower than $c$ or at speed equal to $c$, i.e., within the light cone of the real particle.

- Recombine with (their) virtual anti-particles (if they don't interact as captured in a Feynman diagram) as they coexist always at their $y_{(t f)}^{v}$ position and this fold deactivates as described earlier for EPR entanglement. Here the multi-folds can play a unique role as the allow recombination at distance: through the common exit point, the virtual particle and anti-particle can recombine without ever returning to a same spacetime location. This renders the mechanisms of virtual particles more concrete in $U_{M F}$.

- Interact with something else as described by the Feynman diagram in the background spacetime and the activated folds disappear or higher-level entanglement (3 body or more with respect of the entanglement monogamy principle) would have taken place.

- Super-luminous virtual particles disappear immediately, which can be seen in a continuous model as immediate recombination within or at the edge of the uncertainty region or zero radius multi-folds. They are never allowed to propagate (i.e., the filtering out in the momentum space) beyond a ball of uncertainty around $x_{0}^{\mu}$. This operation is not that different from an ultraviolet renormalization/cutoff [127], and it truly results from the discreteness of spacetime in $U_{M F}$ that will be confirmed later.

The last bullet is a just way for the model to enforces the supra luminosity limit requirement in $U_{M F}$. It is not physical as the real justification comes from the spacetime discreteness in $U_{M F}$. It results from a zero movement, i.e., not leaving a discrete point in discrete spacetime modeled as a zero (or smaller than minimum length) radius fold (which instead of giving infinite effects provides no contribution to the $\mathcal{P} \boldsymbol{I})$. It is a way to understand the approximation of $\mathcal{P} \boldsymbol{I}$ of
$\mathcal{P} \boldsymbol{I}_{S F}$. We expect that in a multi-fold universe this occurs for physical and virtual particles.

We do know that virtual particles are even more controversial in conventional QFT than (real) particles and that usage of them may lead to further confusion. Note that the proposal that we have for virtual particles (emitted in pairs by particles or by energy exciting the vacuum fluctuation), does not in our view counter the opiniated view presented for conventional QFT in [512]. Indeed, virtual particles are proposed here, have no associated creation operator or annihilation operators. They do not become real unless if there is a conventional reason for it (e.g. as in Casimir pressure). Annihilation here is the recombination (possibly in the multi-folds) of a pair. Interaction is absorption by another system of the virtual particle and its entangled virtual partner at the exit point; not the result of creation or annihilation operators that would make the particle reals.

All conservation and Physics laws are respected for the virtual particles; except (or including depending on the point of view) energy conservation. The latter is viewed as respected with positive and negative energies allowed for durations specified by the uncertainty principles, the former allowed as temporary uncertainties. Of course, this model can be seen also a mathematical trick whereby, there are fields of virtual particles, and anti-particles (randomly distributed in the absence of other energy), and now with distribution of emitted (entangled) pairs dictated by energy distributions of all the fields.

The distribution reach spread from each source as time passes, at $c$ for massless particles and at a distribution of lower rates for massive particles; that also limit their range (not just due to the smaller speed but also due to the uncertainty principle).

Entanglement between the pairs will create a "field" of multi-fold effects. All this could be mathematically formulated, and we may do so in future work, but it is not required for the rest of the analysis presented in this paper; as at the risk of incurring the ire of analyses like [512] for muddling the story, using the particle "tool" leads to easier ways to understand what is happening; yes, only if one does not get blocked by arguments like [512] that we do not dispute and, in our view, do not go against, despite possibly the first impression. In the rest of the paper, we assume that this is well understood and do not revisit the validity of explanations and models built on virtual particles: we know there is a pending field version if one wanted to go there. We urge the reader to take that point of view from now on.

Fundamentally, this model implies that type of multi-fold universe $U_{M F}$, where no supra luminous virtual particle exists. No interaction takes place outside the light cone. And virtual particle respect laws of physics; something that is logical when we think of Casimir effects [129] or effects like a friction force of the vacuum explained as mass decreased when a particle is emitted [128].

Note that these considerations may have significant effects on the applicability of the Reeh-Schlieder Theorem [266, $267,268]$ in $U_{M F}$ and the cyclic behavior of vacuum in QFT. Indeed, it is no more true that any state of the universe can 
be obtained by acting locally on the vacuum state ${ }^{60}$. As a result, no correlation or entanglement between space like points can take place between space like points can take place, without other effects. The theorem holds only at a given location for vacuum within its past light cone. Tracking down the implications for QFT and reconstructive QFT and their evolutions is certainly warranted. It does not mean however that, as a result, we may not assume a unique and same ground level vacuum everywhere. We see no reason why that would not remain applicable and it is in fact important to assume so to maintain reconstruct ability of the theory.

\subsection{Gravity out of entanglement}

Repeating the reasoning about EPR entanglement phenomenon, multi-folds are activated and appear surrounding every particle, creating an attractive effective potential in $(1 / r)$ towards any physical particle/entity ${ }^{61}$. As long that virtual particles can be considered as distributed in an isotropic manner, the attraction is isotropic across the cloud of virtual particles. We assert that this is what creates gravity like attractions. Of course, one could argue that only one type of virtual particles, i.e., gravitons, would be responsible for this. It is possible. Yet the analysis presented here would still then account for additional gravity-like effects. To acknowledge that fact we will always assume that gravity can be the combined contributions of all these effects $^{62}$. On the other hand, we believe that:

i) our approach will account for gravity and graviton without the divergence problems. To reintroduce them as an additional contribution would bring back the problems of quantization of gravity/GR.

ii) Our quantized/discrete model will provide a different interpretation for graviton (responsible also for resolving the divergent/renormalization issues and matching the picture painted for gravitons by superstring theory and the AdS/CFT correspondence conjecture). But doing so, it may become rather a quasi particle, or even unphysical.

This does not suggest taking such alternative views where gravitons are (one of the) EPR entangled virtual particles involved in our model. They will rather appear as another effect. Other works can explore these other variations to see if they pan out better. In a multi-fold universe $U_{M F}$, physical particles propagators are as in conventional quantum Physics in $\mathcal{R}_{B G}$ for $U_{\text {real }}$, except that they are filtered to eliminate supra luminous Fourier terms, i.e., paths outside the light cone (hence the green function is convoluted) for the paths in $\mathcal{R}_{B G}$. The effects of EPR entanglement between the virtual particles in $\mathcal{R}_{B G}$ is an attractive potential contribution on all other particles that cross the support domain of the associated mapping. Propagators for relativistic particles are much less spread than in conventional Physics and no space-like exchanges or entanglement takes place. For the rest, the model is just like in the case of EPR entangled particles, except that the multi-folds are now centered on the position of the particle (source of the emission of virtual particles). The energy of the original particle determines the intensity of the flow of virtual particles created and it is directly proportional to it mass.

The effect is therefore directly proportional to the mass of the source. As we saw before, the Path Integral is also proportional to the mass or energy of the entity encountering $\mathcal{D}(M)$. Symmetries between the two particles exist: each generate a similar looking $V_{\text {eff }}$ proportional to its mass times the mass of the other particle (and effective curvatures that results). Symmetry is like a spin 2. Therefore, the effect of the entangled virtual particle surrounding a particle of mass $m_{1}$ generates for a particle of mass $m_{2}$ that cross its $\mathcal{D}(M)$ a $V_{\text {eff }}$ resulting from the contributions of the paths encountered by the second particle:

$$
V_{e f f} \propto \mathcal{K}_{v p} \frac{m_{1} m_{2}}{r=\| x^{a}\left(\operatorname{part}_{1}\right)-x^{a}\left(\text { part }_{2}\right) \|}
$$

Emission of virtual pairs is a priori isotropic: the effect is assumed isotropic ${ }^{63}$. This is really a gravity-like potential.

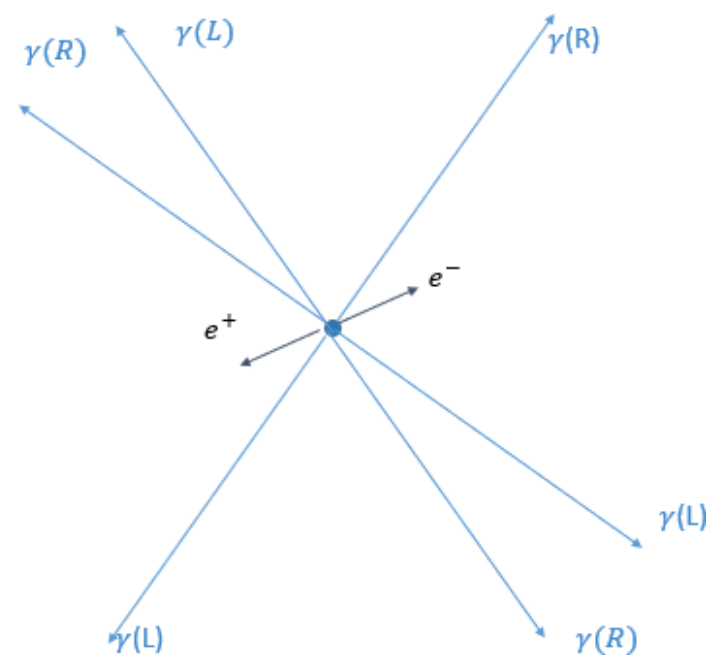

Figure 5: Real particles are surrounded by virtual particles that are created EPR entangled and initiate the multi-folds activations. They result into gravity-like effects for particles crossing the support domain of the mappings for these entangled virtual particles. The effect is isotropic and also involves massive virtual particles at very small scales with similar effects as massless effects, at these scales. Multi-folds live outside spacetime in a tangent dual space.

The reasoning presented above applies for different types of particles: massive and massless; with some variations on the outcome. Let us start with massless versus massive virtual particles. A real particle (massive, massless or a bump in an energy field) is surrounded by virtual particles that can be massive or massless. When they are massless, the range of the effect described above can be infinite. When massive 
particles are involved, a whole range of rather very small scales are involved. The gravity like phenomena associated with the EPR entanglement of the virtual particles exist at long range through massless virtual particle entanglements and it exists in a whole range of microscopic scales carried by entanglement of a whole spectrum of particles and energies at very small microscopic scales. Somehow the carrier of the interaction, through the multi-folds and while awaiting quantization, looks spin-2 respectively massless and massive carriers, gravitons-like. The massive part relates to massive gravity or massive bigravity ${ }^{64}[\underline{347}, \underline{307}, \underline{376}$, 375], which are known to often have ghosts and other consistency problems; yet these seem to have been overcome to a large extent in $[307]^{65}$. However, note the fundamental difference with most of these works: massive gravity-like effects occur only at small microscopic scales; not at astronomic scales, except for neutrinos effects. It implies that at very small scale, the attractive force is actually stronger between particles than predicted solely by the effect of entangled massless virtual particles: additional folds are introduced by these particles and $V_{e f f}$ will grow faster as more massive types of virtual particles can contribute closer to the physical particle. Effects could become significant even if massive virtual particles are harder to generate for a given energy entity; especially as scales will be very small.

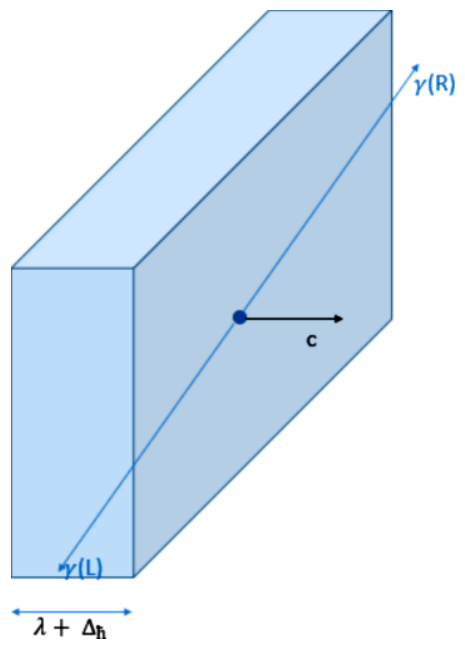

Figure 6: When considering massless particles, only massless virtual particles are involved within the plane orthogonal to the direction of propagation of the massless particle. The effect is rather a parallelepiped because of the need to model a massless particle on at least the width of a wavelength (and the associated uncertainty principle). Massive effects essentially do not appear as massive virtual particles are too hard to generate up to the uncertainty principle allowances. As no reference frame can follow the particle. From a different reference frame, it will appear as a wake railing the massless particle.

The real particles in the processes described above can be massive or massless (or just appears as bumps in an energy field $^{66}$ ). The phenomena differ for massless particles because of the effects of Special Relativity (Lorentz symmetries) and its implications for massless particles that can only move at speed $c$. As a result, massless particles are actually flattened in two dimensions perpendicular to the direction of movement (intuitively, think that it is because of space contraction in the direction of propagation). If we consider an inertial reference frame boosted to speeds close to $c$ to accompany the massless particle, then virtual particles can only be emitted orthogonal to the direction of propagation. Folds are therefore activated tangent to that "plane" and a $(1 / r)$ attractive potential ${ }^{67}$ appears with the plane (or parallelepiped with a width defined by the wavelength of the massless particle and uncertainty principle). This result directly matches the results obtained in GR when trying to estimate the gravity effect of a photon or a massless particle $[\underline{349}, \underline{350}]$. A stream of photons is also discussed in [351] and shows a concatenation of the previous result. The same applies for our model, including the comments about the intensity of the gravity field (double flattening for each segment). As massless particles and the boosted initial reference frame are moving at $c$, massive particles cannot be emitted around the massless particle. As a result, there is no (or negligible) massive gravity-like effect associated to massless particles; only the massless contributions described above: no bi or multi massive gravity is involved in the gravity of a massless particle (except maybe if we included in this the effects of virtual neutrinos, which could be considered quasi massless, at least in it lightest forms). Microscopic torsion could also be introduced by our model. Indeed, with uncertainties of the source and emitted virtual particles, the folds tangent to the virtual particle paths wiggle accordingly.

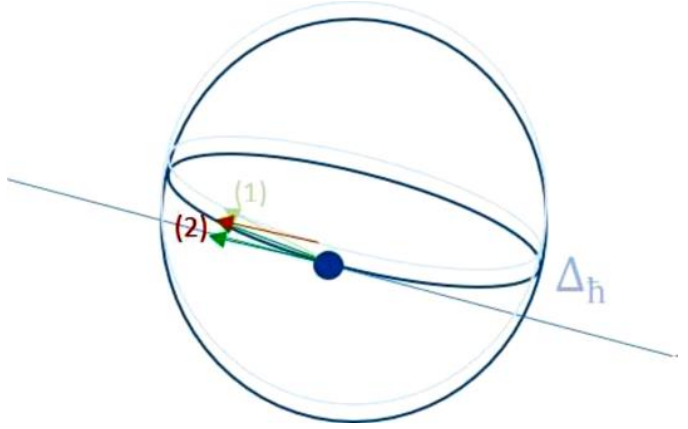

Figure 7: When uncertainty moves the source or an emitted entangled virtual particle, it shifts the fold, or the fold is then shift back or tilted, in order to support later mapping to the same exit point and depending on the uncertainty changes. Between the oscillations, the momentum of the virtual particle entering the fold (path on the fold) is oscillating back and forth between (1) and (2) resulting into a spiral move.

As the source moves around, the trajectory of the virtual particles may be twisted (i.e., with torsion, a lateral displacement needs to be added to come back to same point when moved around by a small closed displacement [352]) near the source particle. These are tiny displacements and torsion effects limited to where matter (the source) is located. They do not propagate, unless maybe for virtual particles as 
virtual torsion or by accompanying the source particle if it moves, and in general one would expect that they average out as do the associated uncertainty fluctuations. However, in the presence of fermions, this may be more significant albeit still very small beyond the uncertainty region ${ }^{68}$. So, torsion is not at all relevant to classic or semi-classic ${ }^{69}$, like $\mathbf{G R}^{70}$, or most non classic situations ${ }^{71}$. Torsion matters because we do expect the lack of gravitational singularities (i.e., in black holes, no cosmologic singularity, support of big bounce solutions) in some models of these theories ${ }^{72}$ [354]), all result from presence of torsion that forces avoidance of the singularity with the displacement over small loops implied in spacetime by torsion: it can never go to a point singularity. So having torsion in our model also implies no singularities ${ }^{73}$ and possible support for big bounces. Yet the absence of such singularities also explains in our views why semi classical model work even at small scales where today we expect that they would probably not apply. More on this later.

To be fair, one could probably as effectively argue that no torsion would actually result from the above: it all depends how we look at how folds and paths on folds are seeded with uncertainty and if/how it is reflected at the entry point from $U_{M F}$ (near the source real particle) ${ }^{74}$. Without the ability to investigate at the scales involved, or to model all the details of the multi-fold dynamics, it is not possible at this stage to say much more. The discrete non-commutative spacetime introduced later allows the introduction of torsion also as a result of the non-commutative geometry [355]. So, it is fair to say: there is most probably torsion and it takes place at very small scales. It is irrelevant for almost everything except for its implication on the absence of singularities, cosmological and black hole, and therefore its support for big bounce solutions. More importantly, pushing semi classical models of gravity to very small scales probably requires at some point making torsion more explicit, hence its illustration here. It is interesting that we can make torsion appear physically quite naturally with the multi-fold mechanisms.

\subsection{Gravity-like symmetries and symmetry breaking in $U_{M F}$}

As discussed previously, symmetries are broken by the proposed process: the deactivation process is irreversible (or away from equilibrium), and so the mechanisms presented in this paper violate $\mathrm{T}$ symmetry. These are not the only symmetry that are violated. Let us list a few symmetry considerations:

$\mathbf{T}:$ The activation and deactivation processes are irreversible and so the mechanisms presented in this paper violate $\mathrm{T}$ symmetry. It agrees with [262], with a completely different reasoning.

P : Because of the absence of right-handed neutrinos (and conversely for anti-neutrinos), the process described is expected to sometimes violate Parity P. A priori this is only relevant for the massive contribution mentioned above, - albeit neutrinos would allow some larger range. However as will be discussed later, we will propose a gravity influenced scenario where right-handed neutrinos (and conversely for anti-neutrinos) may exist for a while during non-interacting ${ }^{75}$ oscillations. This may modify the conclusion. The torsion generation mechanism may also not always be $\mathrm{P}$-symmetric; but should correspond to equivalent Feynman diagrams in the diagram summations. So, there is significant expectation that sometimes P may be violated.

C: For the same reasons, the process, neutrinos left handedness, the process is not invariant under charge conjugation $\mathrm{C}$ for massive gravity at small scale-albeit neutrinos would allow some larger range, unless if recovered by the mechanisms of oscillations mentioned for $\mathrm{P}$ and discussed later.

PT: it is expected to be violated because the T and P relevant mechanisms are rather unrelated.

CP: CP may be violated if torsion affects $\mathrm{P}$ symmetry. Otherwise, if only neutrinos violate $\mathrm{P}$ and $\mathrm{C}$, for massive gravity, CP may be respected by gravity. In general, we expect that CP may be violated, a result in agreement with [358] with his modification of the Hilbert Einstein Action to actions [298, 299] providing the same field equations but exposing explicitly the spin connection (that can also support introducing torsion when matter (fermions and spins) is present) and is, at least to first order, equivalent to Einstein Cartan; again, the torsion saga... This model is the foundation (as classical then semi classical canonical reformulation of GR) of LQG.

CPT: CPT is expected to be violated by gravity, and so by entanglement. This agrees also with [262] with a different reasoning.

Also, it is believed that gravity (with matter) prevents any global symmetry that it be at classical (GR) or semiclassical, as well as at smaller scales $[\underline{361}, \underline{362}]$. In $U_{M F}$, the multi-fold mechanisms have the same implications as soon that folds are activated. These symmetry breaking considerations apply also for EPR entanglement in $U_{M F}$. Yet, many Noether's charges may still be conserved.

\subsection{Vacuum}

The physics of quantum vacuum is extremely involved. Good overviews can be found in $[127,130,25]$. Excited vacuum is described by QFT and its derivatives: it is quantum vacuum bathed in some field [131]. In general, and depending on the details of the fields that you are willing to involve, vacuum is populated with pairs of entangled virtual particles created as random energy/field fluctuations. Well known examples are the electron/positron pairs created in vacuum and possibly polarized in a field (photon). In a multifold universe $U_{M F}$, we can repeat the approach already discussed before. With $\mathcal{P} \boldsymbol{I}_{S F}$, we cut off momentum for the pairs trying to go beyond the light cone as described so far. 
Therefore, space like regions cannot be mutually entangled; idem for correlations. But in our view, this does not prevent having a same vacuum everywhere, with principles or hypothesis of homogeneity or uniformity (e.g. justified by inflation, yet questioned recently in [483]) or isotropy (also essentially justified by inflation and initial isotropy albeit isotropy has been recently questioned [ $\underline{469}, \underline{470}, \underline{483}, \underline{496}]$, not invalidated, though not invalidated).

Yes, some relevant theorems are impacted as discussed in section 4.4. Stability of the (Electroweak) vacuum in $U_{M F}$ will be discussed later. In the vacuum, entangled pairs can be created spontaneously. If it happens at $x_{0}^{\mu}$, a bundle of folds is activated so that the reasoning of the previous session can be repeated. Again, the natural ultraviolet divergence handling via $\mathcal{P} \boldsymbol{I}_{S F}$, and discreteness of spacetime, takes place. In general, these variations come and go and are associated to fluctuations of effective potentials and curvatures. We are recovering the Wheeler's quantum foam picture $[471, \underline{472}]$.

And so, attractive effective potentials in $(1 / r)$ potential appear and disappear continuously in the quantum vacuum. If the momentums are distributed in an isotropic way (e.g. no preferred direction due to other effects) then the effect is isotropic. Effects from different points in spacetime cancel the resulting $V_{e f f}$ on average at every point. However, polarizing or modifying the distribution of the virtual particles (associated to physical particles and to the vacuum) could create local variations of the gravity like effective potentials. Vacuum polarization could be achieved for example via electromagnetism; controlled vacuum entanglement could also be an option.

\subsection{Discussions}

In $U_{M F}$, we saw with equation (8) that the contributions of the folds are proportional to $R$, the Ricci curvature scalar (and $\sqrt{R}$ of the latest spheres/latest folds for multi-folds post integration ${ }^{77}$ ). So, we can also interpret this as stating that, in $U_{M F}$, the background pseudo-Riemannian spacetime is complemented by many additional fold curvatures: summed and weighted by masses/energies of the (source) particles. The direction (of the attractive effective potential) is on the other hand contributing to defining the Ricci tensor (see [363]). From that point of view, it is interesting that it looks like an effective or average variation of the curvature against a background, that is contributed proportional to the mass/energy of the involved particles. This is something that directly matches the expectation if one wanted to recover the effects of Einstein's field equations of General Relativity (e.g. [54]) or Newtown gravity.

The model proposed here is also explaining how a spin-2 process force carrier interaction can involve non spin-2 virtual particles, e.g. other bosons, and fermions; something typically not considered for force carriers and gravity, especially when considering spin/angular momentum conservation in Feynman diagram, as discussed for example in [323]: the mechanism of attaching spin-2 multi-folds, the massless and massive gravitons (to be explained later), to entangled virtual particles emitted near a source. This is a fundamentally different approach ${ }^{78}$ from most conventional attempts to quantize gravity (GR linear perturbation and quantization or superstrings) or reconstructive quantum gravity [243]. Yet it matches, to a large extent, the behavior of conventional spin-2 boson propagation and interaction, albeit the multi-folds do not live in $\mathcal{R}_{B G}$ or 4-D spacetime. It only interacts through entry and exit points and the proposed mapping and as a result it is also Lorentz covariant and background independent. In our view, these differences are also quite important. It is these differences in derivation, interpretation and physics that illustrate how our approach may be able to get rid of the problems of self-interaction, divergences, non-renormalizability and singularities of gravity and quantum gravity ${ }^{79}$, and why gravity is so weak. One can also interpret what we encountered as if EPR entanglements between entities and virtual particles create a sea of folds and are "tangent" to spacetime $\mathcal{R}_{B G}$, with surrounding mappings everywhere and varying everywhere in time depending on how mass and energy is distributed. An entity in $\mathcal{R}_{B G}$ bumps at every point against the folds and their mapping. Bumping ${ }^{80}$ results into attraction defined by the multi-folds and dictated or quantified by $\mathrm{R}$, the Ricci curvature scalar of the fold and the mass or energy of the entity. The density of the multi-folds is determined by mass or energy of the matter in spacetime $\mathcal{R}_{B G}$.

The link between $V_{\text {eff }}$ and R for the multi-folds can be used to derive macroscopically Einstein field equations of GR. Let us sketch a high-level proof and derivation of them that relies on an a priori knowledge of our target. Let us define at every point of the spacetime, $\mathcal{R}_{B G}$, a vector field $\xi^{\mu}\left(x^{\sigma}\right)$ that defines the direction of attraction and $V_{\text {eff }}$ per fold (not per bundle ${ }^{81}$, so it is in $\left(1 / r^{2}\right)$ and must be added or integrated also over all the involved folds in the activated bundles) felt by a test particle (of unit mass or equivalent energy content; no back-reaction) as norm.

Contributions from matter and radiation or massless fields are linearly additive, and they are constructed based on the multi-fold process computing at every point what are the support domains that are crossed (exactly or with uncertainties). So, we add (and compose for $\xi^{\mu}$ ) the resulting effects at that point. We know that the norm of each contribution is in the associated $R\left(x^{\mu}, \operatorname{source}\left(x^{\nu}\right)\right)$ that designates the Ricci curvature scalar felt at $x^{\mu}$ from the different particles or energy bumps ${ }^{82}$ located (in space and in time) at $x^{v}$

$R\left(x^{\mu}\right)$ is similarly the sum of the scalar curvatures. For now, we assume a minimum cutoff distance so that we have no divergences ${ }^{83} \cdot \xi^{\mu}\left(x^{v}\right)$ is proportional to the Ricci tensor while $R\left(x^{\mu}\right)$ is proportional to the Ricci scalar [364, 363]. In any given time, slice, $R\left(x^{\mu}\right)$ (as a measure of $V_{e f f}$ ) is proportional to the green function of Poisson's equation (see equation (21)) which are second derivatives; this implies a direct local linear relation between local energy (mass) density (sources) and the Ricci curvature; something we had already established even without involving Poisson. We now have obtained fields on $\mathcal{R}_{B G}$, that correspond consistently to a Ricci scalar and a Ricci Tensor and that is a function of energy/matter content of $\mathcal{R}_{B G}$. 
We are done! Indeed, as, the equations must be invariant under local Lorentz transformations, something that our discussion will religiously follow till the end, even when ending up in discrete spacetime and something that our process is so far by the use of Path Integrals. Two approaches can be used to then derive the obvious only resulting equation fitting this: a) generalize newton/Gauss law as in [171] (Equation (4.23)) and recover Einstein field equations and Hilbert Einstein Action or, b) express invariance [322, 367] of Ricci Scalar and Tensor as well as a measure of matter's and fields' energy content tensor density linear combination of them with a sum (integral) over an invariant volume: we recover the Hilbert Einstein action [8] with matter/energy terms. The cosmological constant can be introduced later with the usual derivation [8]. In all cases, we recover Einstein's GR field equations ${ }^{84}$, and in linear approximation, Newton's gravity. The fields map to $\mathcal{R}_{B G}$. The expression of invariance amounts to extremizing the area formed by $V_{e f f}\left(x^{\mu}\right)$ (or $R\left(x^{\mu}\right)$ ) along with an action for matter/energy). It is that same process that makes GR match the construction of LGQ spin networks. And the NambuGoto action of strings [366] incorporates the Hilbert Einstein action as mentioned earlier. This surface invariance is also behind the area laws of spacetime horizons and black holes, spacetime thermodynamics (and why a holographic principle could work).

We recovered Einstein's GR equations when we looked at macroscopic effects (extensible to semi classical) and we can see effective curvatures and gravity potentials as averages of time varying contributions at each point of spacetime, as multi-folds tangent to each point, or as time varying extra dimensions growing at each point ${ }^{85}$

Within our proposed model, the $U_{M F}$ is expected to be flat (without matter or energy, i.e., not a really realistic case) or positive. To be negative it would require that the initial conditions lead to a negative curvature for $\mathcal{R}_{B G}$ (not due to matter or to other gravity contributions that those discussed in this paper), or accept exotic negative mass entities. The only positive effective curvature and the always attractive potential explains why gravity charges (i.e., masses) are only positive and gravity is always attractive: it comes from the spherical nature of the folds with positive curvature.

Our model also dispels an argument sometimes presented that argues that GR and Quantum physics would not be not compatible because there would not be a way in GR to account and deal with superposition, where spacetime could be in two different states with different curvatures at the same time. Indeed, in $U_{M F}$, the problem does not exist: the different curvature are in $\mathbf{A d S}(5)$ tangent to $\mathcal{R}_{B G}$ and $\mathbf{G R}$ curvature is effective and obtained as the average/sum of the curvature contributed by all these particles/sources (which match the geometrical interpretation of Ricci tensor and scalar as averages of gaussian curvatures and average of averages $[364, \underline{363}])$. It is therefore possible in $U_{M F}$ to treat spacetime as a quantum entity subject to entanglement or superposition of different possible $V_{e f f}$ or effective curvatures.
An example of what that might mean in spacetime reconstruction is discussed later. In fact, the effects of uncertainties and superpositions are rather exemplified in the form of what is sometimes called as temporal superposition: different masses locations (i.e., different curvatures) may result into different order of events if impacted differently by the different locations [196]. This is also fully compatible with our approach (at least as long that no issues were discovered when checking experimentally the outcome of [196], assuming that we can do that).

The derivation of GR from our model built solely on a framework to explain EPR entanglement is certainly impressive, even if we had already many hints before both from the $(1 / r)$ attractive $V_{e f f}$ attractive gravity like potential and the trends in Physics that entanglement and spacetime are related as well as the $\mathbf{E R}=\mathbf{E P R}$ conjecture. Albeit derived from a different type of reasoning that just computation of a Lagrangian or an amplitude, our result reminds the claims of fame made by string theory. Indeed, for strings, the main validation so far (see for example [370, 20]) comes from the apparition of the graviton, despite originally not trying to model it $([\underline{371}, \underline{372}])^{86}$. The initial explosion of interest in strings started with that discovery of gravitons matching linear perturbations of GR.

It is ironic that entanglement, one of the most unique feature characterizing Quantum Physics, is the source of GR, and gravity; considering how it is always stated that GR and Quantum Physics would be incompatible or that gravity would collapse the wave functions and destroy superposition and coherence. It is quite a different outcome isn't it.

$\mathcal{R}_{B G}$ can be initially curved or flat. It is again mostly a question about what our starting point is, and what kind of modelling we are targeting. A curved $\mathcal{R}_{B G}$ may be a good way to track just the additional effect of new particles. But if we start from scratch with an empty spacetime $\mathcal{R}_{B G}$, then the model above would imply no curvature for $\mathcal{R}_{B G}$. As entities with mass/energy are added or appear, a sea of folds and mapping appears defined by that density and evolving with this matter/energy density ( $\sim$ back reaction) and as a result a positive effective curvature (described at quantum, semi classical and classical scales by GR). The effects of these folds add up and on average appears equivalent to the effective average curvature. As a consequence, we already see that no divergence or singularities will occur if we assume that any curvature extends up to the uncertainty region around the particle ${ }^{87}$. It is therefore possible, logical and expected that curvature is essentially a(n) pedagogical illusion: spacetime $\mathcal{R}_{B G}$ could remain flat in a multi-fold universe $U_{M F}$, and the multi-fold phenomena or averages of curvatures give the impression of curvature ${ }^{88}$. While different in terms of the resulting model, it also relates to the attempt done by Rosen to treat $\mathbf{G R}$ as a field over flat Minkowski space instead of a geometrical phenomenon [365]. Similarly, Gupta modeled effects of GR in flat space as infinite series of perturbation of the Lagrangian density in flat space, which he interpreted as a property of a spin-2 carrier [356]. Later, we will summarize the effect on attempting to model a Lagrangian for our model and will see 
that indeed it will require nontrivial changes to what would happen in flat space.

$U_{M F}$ could also be primarily curved for whatever reason or initial conditions, and the folds then describe how $U_{M F}$ is further perturbed ${ }^{89}$ and back reaction to additional matter or energy. De facto, we have shown that our model for $U_{M F}$ is background independent [25, 249, 248], i.e., it does not assume a fixed background (see how we can change the conditions) and by definition is built by the sum of the contributions of all the entities and so dynamic by nature at all time; something believed by many to be key to ensure correct modelling/avoidance of graviton self-interactions $\mathrm{s}^{90}$ [248], and essential to align with GR that we need to recover at semi classical and classical scales.

We have provided a reasoning for introducing multi-folds and their kinetics and dynamics in $U_{M F}$ in order to satisfy that reasoning. We did not claim that other approaches addressing our requirements cannot be encountered. In fact, another way would be to start from GR in (some) spacetime and try to build such solutions. If they exist, they probably would differ from the approach above by the fact that the Hilbert-Einstein (adapted suitably to the dimension) would apply (e.g. think of ER bridges if $U_{M F}=\mathcal{R}_{B G}$ ) and/or that interactions can take place within the folds; something that we have not allowed so far (because we assume every particles that folds are essentially hard partitioned instances (multi particles); structures enforced by the mappings). Studying such and other variations could of great interest, and for future work (See [513] for more recent developments).

\subsection{Quantum Fields}

Fields are more complicated to deal with in our model which, so far, relied on the notion of particles. There are several challenges associated to QFT for our multi-fold universe $U_{M F}$ model if we were to use conventional QFT as is. We list the most problematic ones:

- Conventionally, quantum fields are non-local. They can be generated everywhere from the vacuum at one spacetime point, and as a result can have the field at any point in spacetime entangled with any other point in spacetime. In other words, entanglement is not local and extends (at least as correlations) to spacelike regions. The allowance of supra luminous virtual particles outside the light cone leads to this (or results from the above) even if Lorentz symmetries and invariant considerations save us so far $[\underline{125}, \underline{126}]^{91}$.

- Conventional quantum fields are often considered as incompatible with the notions of particles [15]; typically, because of the history and processes of second quantization that do not preserve the number of particles, something inherent to the design of QFT $[\underline{72}, \underline{16}]$. The problem is exacerbated by the phenomena of creation of particles in curved spacetime [171].

- Furthermore, if a particle is assumed associated to a bump of energy in a conventional field, then in QFT it is almost immediately spread everywhere $[\underline{73}, \underline{74}, \underline{78}, \underline{79}$, $\underline{80}, \underline{55}, \underline{123}, \underline{26}, \underline{133}, \underline{134}, \underline{272}]$. As a result, in conventional QFT, particles cannot be well or lastingly localized. This is "unphysical" from a particle point of view: we know they exist... Yet, the notion of particle does not seem to make much sense in QFT [26, 273]. The interpretation of one of its biggest successes (Feynman diagrams), explicitly relies on particles, virtual and reals, and all their possible interactions. One way to understand the apparent contradictions of this latter observation is that Feynman diagrams are part of computation methods in perturbative algorithms. As we pointed out in the present list, energy bumps can be seen as particles in conventional QFT but only for a very small amount of time and in a very localized region as allowed by uncertainty principle; so that they do not "spread everywhere" in ways that prevent them be tracked and in as much that we allow the particles to disappear (When annihilated) and new ones to appear (when created). This why there is no actual contradiction and Feynman diagrams work, and in fact work so well! Yet beyond these very small perturbations, particle concepts are lost, and Feynman perturbative methods fall apart. It does not matter; it was not its purpose. But again, this shows that there is a clear concept of particle in conventional QFT; it just does not last long. It also reminds us that QFT is the result of computational techniques, it is a model. It is ok that there are things QFT is good at modelling, and things it is not good at modelling. And yes, surely, many are shocked by such a statement.

- One could argue that conventional QFT is actually rather a statistical physics theory and that is also why its methods (e.g. field/functional Path Integral, CFT and holographic duality [141]) apply so well to statistical physics and solid states physics ${ }^{92}$. Conventional QFT does not track down well at all a particular set of entities. It predicts well probabilities of events and what events can or will not take place. It also cannot well handle entanglement beyond the statistics of entanglement entropy (e.g. à la von Neumann) [27].

Therefore, and unfortunately from our point of view, conventional QFT does not align well with our approach in this paper, nor our needs. We have so far relied on discussions in terms of particles and banning supra luminous velocities (interactions or propagation beyond the light cone) and, as a result, banning entanglement between space like regions. Considering the success of QFT exemplified for example by QED, QCD and the Standard model, it seems quite a challenge! However, Quantum Physics is a model and an approximation designed to address particular phenomena. Its model may not be well suited for all use cases.

In addition, after all, particles exist even for long time or over long distances, as seen in particle accelerators, or especially in experimentations of the Standard Model. Even Path Integrals, scattering matrices and Feynman diagrams interpretations of QFT immediately reduce to interactions between physical and virtual particles. In fact, perturbative QFT and Feynman diagrams methods really amount to 
counting these different possible interactions for a given Lagrangian or Action which describes particles modeled by fields (tracking a few particles, their interactions and allowing particle creation and annihilation).

As a result, we assert that, for $U_{M F}$, there is no problem that fields are showing that there does not exist notions of particles [15]; but rather that particles and fields are different approximations or facets of reality. Both are meaningful, and both have their limitations. In fact, when dealing with conventional QFT, there are "recipes" or ways to handle particles. Examples include:

- Particles can be approximated as isolated in big enough boxes [25].

- Particles are actually present in QFT, and in Feynman diagrams but not just through Feynman's diagrams [24]. However, one needs to follow the particles (unless and until annihilated) and not be distracted by particle creations and annihilations that cause much of the trouble.

To this we add:

- The absolute requirement of no supra luminosity, significantly resolves the problem of dispersion or leakage of the particles. Many of the issues with relativistic frameworks directly come from computing without such a constraint, as discussed earlier.

In that context, our work suggests that, in order to capture the phenomena in $U_{M F}$, that we have described in this paper, QFT needs also to explicitly model entanglement between particles ${ }^{93}$. It means also not just correlations or multiple point correlations in QFT, or statistical entanglement as typically modelled by von Neumann's entanglement entropy ${ }^{94}$, entanglement Hamiltonians or density operators [27]. When modelling that way; it is already one level or scale above what is really happening and one can only at best hope to obtain a statistical physics or thermodynamic model of the phenomena. There is a gap in conventional QFT models. We need to rethink how to add these concepts with or without modelling particles. Until it is done, conventional QFT cannot well model the phenomena in $U_{M F}$ and therefore it probably cannot account for gravity, at least for the portion contributed by the phenomena we described. and EPR like entanglements. We believe that this statement also applies to supersymmetry, superstrings and all derived theories ${ }^{95}$. Finally, and as harped already by others, conventional QFT (and supersymmetry, supergravity, superstrings, and all variations) needs to include a way to add background independence to address its divergence, renormalization and graviton self-interactions problems, which again appear because they need to model endless series of back reactions that background dependent models can't capture.

This is our message: address these points with the principles above, admitting that in this paper we do not describe how to do it, and the gap to model quantum gravity may become significantly smaller! In fact, we also suspect that all the challenges and con- fusions around correct expressions for
Einstein's stress-energy-momentum tensor (symmetric or not, à la Belifante-Rosenfeld or not, Canonical from Noether's theorem or not, with or without the spacetime contribution) [494, 348], fundamentally result from the difficulties of dealing within spacetime with the multi-fold effects that live outside, and may not play the same role in terms of the (peudo-) tensor and its conservation. Also, it is probably why massive gravity leads to so many problems $[307, \underline{376}, \underline{375}]$ in conventional classical Physics, or quantization.

In $U_{M F}$, there is a need to revisit, with supra luminous absolute limit, the principles behind the derivation of the Reeh-Schlieder theorem [266] (as discussed earlier), the Malament no-go theorem [26] (translation, as used in the proof, with the supra luminosity condition, weakens the argument) and the Hegerfeldt theorem [133, 134]. When computations filter the contributions outside the light cone, the proofs do not hold any more. Reasoning as the ones presented in [272], that are already addressed by the design of $U_{M F}$ ), are also to be revisited. The proofs of invalidation are immediate from eliminating paths outside the light cone. And so, in a multi-fold universe $U_{M F}$ :

- Particles can be localized in $\mathcal{R}_{B G}$ and evolve.

- Vacuum excitations at a point location cannot generate space like states, entanglement, coherence, or correlation space-like elsewhere in $\mathcal{R}_{B G}$. Again, it does not mean that vacuum cannot be the same everywhere if only one vacuum lowest energy level (ground) exists; but there may also be situations where it is not the case and stability (as in Electroweak vacuum stability) is to be addressed, which we will do.

- In $U_{M F}$, QFT does not imply entanglement of vacuum state everywhere with everything.

\subsection{Semi-classical Gravitons, before quantization, as multi-folds attached to EPR entangled particles and $V_{\text {eff }}$ fluctuations}

The notion of graviton may solve the problem of modelling suitably entanglement in QFT, by simply reverting to adding interactions with gravitons to all what is EPR entangled. We have discussed above how multi-folds have a spin-2 symmetry and can be viewed as living outside $\mathcal{R}_{B G}$ while creating a fluctuation or wave of attractive $V_{e f f}$ in $\mathcal{R}_{B G}$ that also amount to effective curvatures (scalar + direction). Let us analyze what gravitons may or may not be in a multi-fold universe $U_{M F}$. We follow the traditional linearization procedure of $\mathbf{G R}$ as investigated first by Matvei Bronstein $[\underline{368}, \underline{369}]$. It is now automatically assumed and accepted by all and described for example in a modern form in [23] (see also [356]). Accordingly, the metric is perturbed, typically from a flat state described by Minkowski metric. As a result, the traditional observation is that the perturbation propagates at $c$ as a change in the metric, and hence curvature. It corresponds to a spin-2 symmetry and appears to be described by a massless boson relativistic (QFT) wave equation and Lagrangian density, and can be used to recover 
Einstein's GR field equations (See also [323]). However, in $U_{M F}$ the story is a bit different: the perturbation of the metric implies a perturbation of the Ricci tensor and scalar, and it must be contributing a positive effective curvature. We know that this results from a particle or energy bump in $\mathcal{R}_{B G}$. As it appears the multi-folds result into attractive $V_{\text {eff }}$ that appears or is perturbed. The d'Alembertian wave equation, matched to the energy momentum tensor (of matter), corresponds to a wave of $V_{e f f}$, in norm and direction, due to the dynamics of the multi-folds based on the perturbation:

(i) due to entangled virtual particles emitted near the bump or

(ii) due to entanglement between particles created at the place of the bump.

The process has spin-2 symmetry and behaves as if the wave was massless for entanglement of massless virtual or physical particles (supporting gravity and EPR entanglement) but also massive when the (virtual) particles involved are massive. A priori no particle is exchanged in $\mathcal{R}_{B G}$, but the exchange is rather in the tangent and dual space where the folds are living. Folds do not interact with each other, except maybe at entry and exit points (with mappings). So, with our current model, multi-folds as gravitons, do not self-interact even if the equations describing its effect in $\mathcal{R}_{B G}$, and the tensor propagator carrying charges (masses) would a priori imply that it does. Until and unless we are forced by other considerations to add interactions between folds, the theory will no longer suffer of renormalization and divergence problems. Background independence, as we managed to model it, seems to indeed make all the difference as theorized in [248]. With our approach and background independence, we can drop self-interactions. It probably provides hints on how other theories can formulate entanglements at their level or scale ${ }^{96}$.

One could take exception to our proposal that the EPR entanglement of virtual particle generates gravity, despite the analysis done so far and the results that we recovered. It would amount to insisting that a separate carrier (the graviton) lives in spacetime and carries the interaction. That is exactly the linearized gravity perturbative approach with all its problems. Our approach addresses these problems by eliminating that option and explaining gravity differently. That is really the cusp of our proposal once we have discovered that, in $U_{M F}, \mathbf{E P R}$ entanglement creates an attractive potential in between the entangled particles. Doing so, the effect of the attractive $V_{\text {eff }}$ (wave) appears and propagates in spacetime. It is that effect that linearized/quantized GR associates to gravitons ${ }^{97}$. For us, the graviton is rather the effect of the multi-fold mappings for the virtual particles emitted by the source.

It is also possible that the model we propose is correct but rather only associated to the propagation of $V_{\text {eff }}$ in spacetime and not due to virtual particle entanglements (other than gravitons); it is not clear at this stage if the interpretation would behave differently in spacetime. It suffices to say that multi-folds seems more like a kind of quasi particle, than a particle. It is also possible that all these contributions exist ${ }^{98}$.

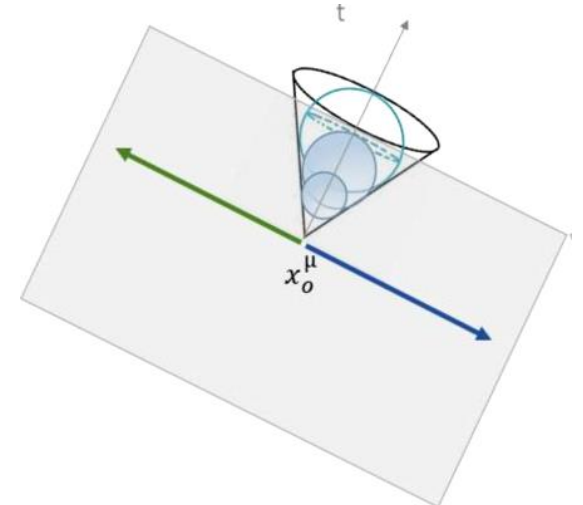

Figure 8: Cones of folds built by wrapping the spheres evolving with $t$ tangent to $U_{M F}$.

Until one worked in a universe like $U_{M F}$, it was logical that models in $\mathcal{R}_{B G}$, modeled the gravitons in $\mathcal{R}_{B G}$; there simply was no other place where to put the dynamics. In this paper we will not further consider these variations.

\section{SELECTED IMPACTS ON PHYSICS}

\subsection{Contributions to the Anti de Sitter Saga}

The fold-tori of 3D spheres around a particle in $U_{M F}$ are sets of tori wrapping sets of spheres each evolving into cones along a time axis, all tangent to the time \& tori/momentum space. This is shown by figure 8 . These cones are always present for physical and virtual particles as they propagate and interact ${ }^{99}$. In a multi-fold universe $U_{M F}$, particles are surrounded in their proper reference frame (i.e., following them), and at the level of the activated folds $\mathcal{R}_{F}\left(x^{\mu}\right) \in$ $B_{\text {activ }}\left(x^{\mu}\right)$, by a portion of tangent universe looking a lot like an (or a set of) anti de sitter space(s) with the t parameter as extra time, or scale, dimension. Indeed, for a given momentum to handle, the spheres, evolving in $t$, live at in a 4-D spacetime with a (1(time),3(space)) type of metric: they are travelled by the paths computed in the Path Integrals on $B_{\text {activ }}\left(x^{\mu}\right)$, in time and in space (and it is repeated for all possible momentum). So, $B_{\text {activ }}\left(x^{\mu}\right)$, can be seen as tangent and dual to spacetime at each particle $x^{\mu}$ (where $t$ is $\tau$, the proper time for the particle) in a bigger anti de sitter (with 2(time) and 3-(Space) dimensions) or $\mathbf{A d S}(5)$ space [135].

And so, $U_{M F} \subset\left(\mathcal{R}_{B G} \times \mathbf{A d S}(5)\right)$, where $\operatorname{AdS}(5)$ has isometry group $\mathrm{SO}(3,2)$ symmetry, and is isotropic.

The apparition of AdS(5) around every physical or virtual particle, tangent dual to spacetime $\mathcal{R}_{B G}$, is certainly worth pausing, even if just the result of the time parametrization of the folds on which the spacetime paths are computed. In our model, nothing imposed that (multi-)folds in $\mathbf{A d S}(5)$ or that AdS(5) spacetime follows GR. The first fact emerges, the second remains possible, but it is not required. It is interesting that Anti de Sitter spaces are also the maximally symmetrical solution of Einstein equations with a negative curvature (as a $(1,3)$ time-space) and with a negative cosmological constant [136]. A negative curvature is something that our approach cannot generate by the EPR 
entanglement multi-folds processes. It may be for good reasons, as we know that AdS spacetime solutions of Einstein GR equations are unstable with matter resulting into black holes [451]: such spacetime may never physically exist. Even more interesting is that many physics models and computations, including those trying to extend QFT with renormalizable theories and CFT [44, 274], (superstrings, supergravity and quantum gravity have been developed in greater details in, or in relationship with, Anti de Sitter spaces ${ }^{100}$ ). Alternatively, projections (e.g. holographic principles) or infinite asymptotic behaviors are considered as modelling our spacetime; but they are mostly conjectures within $\mathbf{A d S}$ (5) and especially outside it (e.g. in positive curvature spacetime, like asymptotic dS (de Sitter) universes).

Anti-de sitter should ring a bell for anybody familiar with CFT and strings. Indeed, with $\operatorname{AdS}(5)$, we encounter the famous duality conjecture between $N=4$ (maximally) Supersymmetric Yang-Mills (SYM) in four dimensions and type IIB string theory on $\mathbf{A d S}(5) \times S^{5}$ [142] (we also note it sometimes with + to indicate additional spaces to consider), where a geometry of a $N=4 \mathrm{SYM}$ can be seen as the holographic representation of $\mathbf{A d S}(5)$; leading to an intersect of superstring theories and CFT, i.e., renormalized QFT. It is the "AdS/CFT correspondence" conjecture that has had many impacts across physics; everywhere QFT, or rather CFT theory applies [275], including solid states [141]. The original paper is $[138]$ (see also [137, 276] and simplified overviews can be found at: $[\underline{139}, \underline{140]})$.

Although just a conjecture, it has been repeatedly validated as consistent or providing useful, and sometimes unintuitive results in many settings. Essentially QFT/CFT describing particles in a $N$ dimensional space (Yang Mills $N=4$ maximally supersymmetric) can be seen as projections of superstrings and string theory living on the inside of $\mathbf{A d S}(5)$; and yes, this include quantized gravity (strongly coupled). In fact, it has been shown that, under particular conditions of large degrees of freedom and strong coupling then (strongly coupled) gravity in $\mathbf{A d S}(5)$ (+ other dimensions) can be projected onto weakly coupled CFT (conformant field of QFT) in a 4 dimensional Minkowski pseudo Riemannian spacetime without gravity. The conformance conjecture comes from the hypothesis of angle invariance (even if scales can be changed). A good, simplified overview is presented in [141]. Higher dimensions cases can be found discussed in $[142, \underline{139}]$

Our model illustrates links between $U_{M F}$ and AdS(5). From this we will later discuss the CFT/AdS correspondence for $U_{M F}$. We will also show its implications for the string landscape vs. swampland [61] and, demonstrate the holographic principles in its suitable form for $U_{M F}{ }^{101}$ and $\mathcal{R}_{B G}$. So far, the existing party line in Physics is that anti de sitter universe gives some good approximations (with exact forms), analogies, intuition, mappings and understanding of how (particles - remember, as explained, conventional QFT has a problem with them; but we address it in our multi-fold universe), QFT/CFT and superstrings (in AdS(5) or higher dimension versions) concepts relates by projecting the superstrings that describe them onto the boundary surface of
AdS(5) (i.e., our universe). Interestingly, while much is still work in progress, a string theory of gravity in the Anti de Sitter spacetime of dimension D can be a (Q-) CFT (or particle) theory without gravity in the lower (D-1) dimension spacetime. Yet in our multi-fold universe $U_{M F}$, gravity may be explained with multi-folds and anti de sitter spaces naturally appear tangent wherever and whenever particles are created, present and entangled. An interpretation is that physical and virtual particles are being surrounded by such AdS(5), resulting from the activated bundles of folds (i.e., $\operatorname{AdS}(5)$ is embedding them) $B_{\text {activ }}\left(x^{\mu}\right) . \mathcal{R}_{B G}$ is tangent to $\operatorname{AdS}(5)$ and it represents the conditions required for Yang Mills conformant fields as projected images of $5(+5$ or 6$)$ dimensional superstrings. This happens at the location of any particle and for every type of particles, and so, superstrings could be hinted by the $B_{\text {activ }}\left(x^{\mu}\right)$ plus additional superstring properties determining what particle is associated to it (e.g., think of the string vibration). So fundamentally, we invert ${ }^{102}$ some of the duality use cases and infer superstrings in $\operatorname{AdS}(5)+S^{5}$ (possibly+1) ${ }^{103}$.

Links to superstring-based gravity is a different question. In the same spirit, we note that (multi-)folds reminisce of closed strings as gravitons encountered in superstring theory and living in negative curvature spacetime (e.g. $\mathbf{A d S}(5)$. Most mathematically developed models, and per the string landscape analysis, seem to restrict viable realistic superstring models to negative curvature spacetime (e.g. [94]), in agreement with our findings. So, potentially, the multi-fold mechanism can then be seen as a way that brings anti de sitter spacetime around any particle and could explains how their creation and behavior can be modelled by superstring theories. For example, the $\mathcal{R}_{B G}$ could be a Dbrane where the string associated to the particle attaches to characterize it in the local AdS manifold ${ }^{104}$. A Multi-fold universe with positive curvature, and gravitons, or superstrings, living only in negative curvature AdS(5) spacetime, as predicted and restricted by our model, and superstring theory shows surprisingly a lot of consistency between the conclusions of approaches that are, or at least appeared, initially so different. Furthermore, we just established that $\mathcal{R}_{B G}$ can be flat, i.e., without gravity, and multi-folds or gravitons live outside (i.e., in $\mathbf{A d S}(5)$ ). This starts to also look a lot like the AdS/CFT correspondence conjecture. If $\mathcal{R}_{B G}$ is flat without gravity and gravity (gravitons) are in AdS(5) impacting $\mathcal{R}_{B G}$ through the folds and mappings, then mappings involved in the fold mechanism may also relate to the correspondence and/or holographic models.

And, we have just recovered the weak/no gravity in $\mathbf{A d S}(5)$ duality. In fact, with gravity in $\mathbf{A d S}(5)$, yet the effect of gravity through the attractive effective potentials. This way, we have a renormalizable story for gravity while no gravity in spacetime! One could interpret this way why we meet CFTs instead of QFTs in the AdS/CFT correspondence conjecture and see that $V_{e f f}$ is the result of a holographic effect (implemented by the mappings).

These thoughts on superstrings in the last few paragraphs, shows hypothetical ways for compatibility, or possible links between these theories, and multi-fold universes. Nothing in 
the multi-fold universe model requires that these other theories be correct. But it is captivating to see $\mathbf{A d S}(5)$ and superstrings become relevant to $U_{M F}$. We also note that the angular invariance under scaling $[141, \underline{276}]$ is exactly what is also behind our derivation of $\mathbf{A d S ( 5 )}$ tangent to any particle: the time-like parametrization of the folds can be seen as changing the scale (which changes lengths but not the angles). As a result, position of the folds is linked to its growth speed, and speed of the attached particles. This has strong implications that we will review later as in our $\operatorname{AdS}(5)$, at least at the level of multi-folds, we will have nonzero commutators between operators of coordinates (the spacetime positions): a sign of uncertainty, when quantized as well as non-commutative geometry, something also met in superstring theory, and often a sign of a discrete spacetime.

\subsection{Nonlocality}

Bell had already argued and provided a formalism that demonstrates nonlocality in quantum physics $[\underline{5}, \underline{466}, \underline{258}$, $\underline{100}, \underline{257}, \underline{265}]$. We have discussed it in section 4.1. However, Bell does not explain the source of non-locality. In a multi-fold universe $U_{M F}$, non-locality is resolved by the activated folds that links seemingly unconnected EPR entangled particles: by allowing paths outside $\mathcal{R}_{B G}$, information can be exchanged between particles ${ }^{105}$ distant in $\mathcal{R}_{B G}$ but located at the same point $y(t)^{v}$ in one or multiples of the folds in $B_{\text {activ }}\left(x^{\mu}\right)$. All this without supra luminosity. de Broglie and Bohm have tried to explain the non-locality concepts and the challenges in causality with the notions of quantum potential (especially for relativistic particles) [466, 144]. Although not reviving the hypotheses or derivation of [144] and the works described in [466, 144], our approach can be seen as deriving nonlocal quantum potentials to Schrödinger, Klein Gordon and Dirac equations and demonstrate non-locality as a result of these nonlocal quantum potential. Interestingly, and following our motivation for the folds, the approach to provide nonlocality is by providing locality in the folds ${ }^{106}$; thereby offering an imaginative way to reconcile the apparently irreconcilable.

As an alternate variation to our approach of multi-fold universe $U_{M F}$, we already admitted that one could try to find other fold mechanisms (different properties, kinematics or dynamics or spacetime where they appear) (see 4.8) or simply different mechanisms. For example, we could find other ways or postulates that generate an effective potential $V_{\text {eff }}$ between particles in $(1 / r)$ without multi-folds in order to explain gravity. Yet, non-fold-based mechanisms may not be linked to effective curvatures without some additional considerations and they may not

i) imply or explain a link to entanglement ${ }^{107}$

ii) provide the same type of resolution to nonlocality.

Of course, (i) could be maintained (i.e., introducing the alternative mechanisms also for entanglement) if we forsake the desire to give an intuitive explanation to their introduction.

\subsection{Multi-folds and Spin}

Although widely accepted and validated as an essential quantum property of particles, individual and composite, spin, and its origin still remain to a large extent mysterious [388]. Attempts at explaining it today still lead to different models. In particular, it is common that spin be justified as a purely relativistic concept emerging from adding relativity to quantum mechanics to make it emerge from the Dirac equation, through the spinors. That explanation is not necessarily the full story. Yes, Dirac and Klein Gordon equations can be derived by imposing that Lagrangian behave well under Lorentz transformations (rotations and boost) and constructing the group representations. Yet, it has been shown that spin can be derived the same way from manipulating Schrödinger's equation to linearize it (just as Dirac equation linearizes the Klein Gordon equation) [390]: it appears that spin more fundamentally results from enforcing first order spatial derivative dependency, and fermions/spinors can also appear as representations of Galilean transformations.

It is not all, different analysis of relativistic quantum mechanics/QFT also lead to different views when trying to go beyond, i.e., more physical, the point of view that spin results from angular momentum conservation and representations of Lorentz transformations, and that this is all there is to know. At best, it results into considering that spin is an internal or inherent (i.e., non-orbital and nonmechanical/kinetic) angular momentum. It is widely accepted; but it does not explain everything; it is just a phenomenological and mathematical explanation. Yet it has been shown that spin can be viewed as a circulation of energy or momentum in the wave function [ $\underline{391}, \underline{392}, \underline{393}, \underline{394}$, i.e., a physical effect. Not much more than the wave function can rotate in a point particle world. Note that $[394]$ presents compelling arguments that this point of view works in recovering properties of the electron spin.

A way to picture these results could be as the rotation of the wavefunction itself; but what if the wavefunction is unphysical as usually admitted? In a multi-fold universe $U_{M F}$, the folds surrounding a particle could be rotating spacetime locally. This is illustrated in figure 9, where we can see that different spins can be obtained with different ways to distribute of entry of the folds. If patterns are followed this way (or other variations), we could have a physical interpretation for the physical momentum or current discovered above ${ }^{108}$.

Interestingly, it allows us to treat spin as a different kind of rotation that the non-point particle while still having a physical meaning. The entry point behavior then relates closely to the torsion at entry, which also hints that, as mathematically known, torsion and spin relate and can couple or interact. Yes, the explanation is still more a handwaved curiosity, but it aims at emphasizing that this multi-fold spin model is not that implausible. 


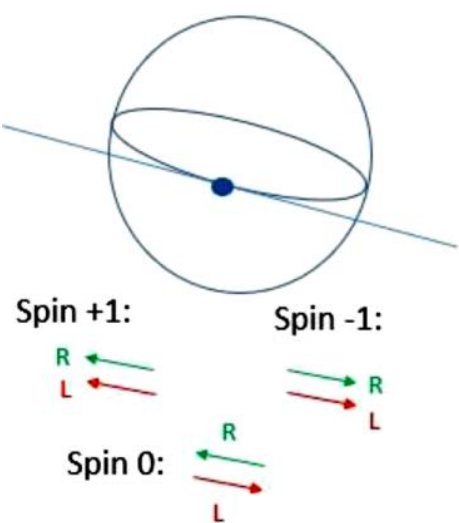

(a) Bosons

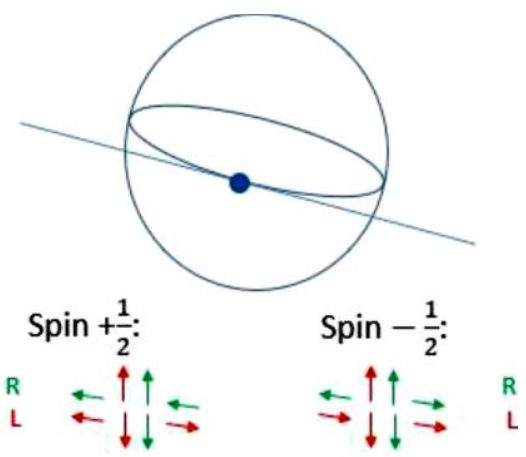

(b) Fermions

Figure 9: It illustrates possible patterns of distribution of how entangled virtual particles could enter paths in the activated folds to provide different spins (by rotating the spacetime around the folds). The virtual particles are dominated by virtual photons with two possible polarities $(R$ and $L$ ). Entries can be along the grand circle along the direction of propagation of the virtual particles or orthogonal (to have independent contributions). With a not yet quantized model for gravity, this can be in any direction as virtual particles are emitted in an isotropic manner and the criteria for one pattern to another are not explained other than for symmetries of (a) $360^{\circ}$ for bosons vs. b) $720^{\circ}$ for fermions, where entry patterns and polarizations of the photons have to be considered.

Unfortunately, with just a semi-classic theory, and this way, we cannot detail why spin is quantized, unless if related to discreteness of spacetime and hence momenta, nor when a pattern is to be applied. That is, at this level, probably what is intrinsic to a particle (type).

\section{MACROSCOPIC AND OTHER ENTANGLEMENTS}

In this section, we extend the discussion of entanglement to macroscopic or macroscopically manageable systems. This is what is sometimes called quantum matter [141], defined as forms of matter where the effects of entanglement are manifest at the macroscopic scale, and with entirely different physical properties than when no macroscopic entanglement takes place [141]. Quantum matter covers superconductors, superfluids, Einstein Bose condensates, strange metals [285], etc. A good list can be found in [141].

Good examples would most probably come from states of matter that display behaviors that directly result from quantum physics and entanglement. For example, Bose Einstein Condensates [145] have been shown to be significantly "entangled", no matter what their realization is [120].

One of our hopes is that we may be able to predict behaviors that can be validated experimentally, or could at least hint if a multi-fold universe could match $U_{\text {real }}$. Of course, the easiest way to check if EPR entanglement generates gravity like attractive potentials would be to EPR entangle macroscopic objects. The size of what can be entangled is increasing rapidly $[\underline{374}, \underline{373}, \underline{473}]$, and so, at some point, it may become possible to have system large enough to offer a detectable gravity-like effect.
A more complete compilation and analysis of relevant entanglement examples and how they are impacted in $U_{M F}$ is for future work. Many more examples are worth discussing beyond the few compiled below.

\subsection{Superconductors}

Good examples of macroscopic entanglement effects are superconductors where Cooper's or BCS pairs of entangled electrons (low temperature) (created by phonons interactions across macroscopic lattices) [146], and Bose Einstein Condensation of (preformed) BCS electron pairs and among pairs and pairs of pairs (e.g. possibly for higher temperature superconductors), provide the superconductivity behaviors $[\underline{141}, \underline{147}, \underline{148}, \underline{149}]$.

Low temperature superconductors typically involve BCS pairs. Higher temperature superconductors cross over from phonon-based lattice wide scale (i.e., many cells of the lattice as much as 104 times the lattice interspacing) BCS pairings to BECs of tight BCS pairs (with an interim BCS-BEC cross over). The exact mechanism(s) of formation of these pairs in higher temperature superconductors are not yet agreed upon nor is it explicitly expected that they would be the same in the different types of high temperature superconductors [151].

They include models with more complex phonon exchanges than just lattice vibrations as in low temperature BCS, spin or magnon coupling, sites on super atoms [150], etc., to support entanglement and formation of BCS pairs in BEC of the higher temperature superconductivity. Because pair entanglements are tighter, the attraction mechanisms are stronger (stronger coupling) than in the lower temperature BCS cases, which renders perturbative methods more problematic, coupled with the renormalization problems 
encountered with the related Anderson-Hubbard model [152].

This has led to the introduction of new models, and in particular the notions of holographic superconductor models where an analog to the AdS/CFT duality is exploited by analogy to what we have described earlier for QFT to model strongly coupled fields (BCS Pairs in BEC) with low coupled fields and a gravity effect in an (Asymptotically) AdS universe; something that can be completed with variational principles (because of the low coupling) [141, 153, 154]. It can reproduce many of the thermodynamics and phase transitions observed in high temperature superconductors. It is also interesting that this also showed that low temperature BCS with elemental superconductor as associated to s-wave electrons, while higher temperatures also involve p-, $\mathrm{d}$ - and $\mathrm{f}$-waves resulting into $\mathrm{p}-\mathrm{d}$ - and $\mathrm{f}$ - symmetries of the pairing. The London effects associated to superconductor can be seen as involving symmetry breaking (a la Higgs ${ }^{109}$ ) with massive photons and penetration depth effects [286, 157].

For the purpose of this paper, we assume that low temperature superconductors are characterized by macroscopic lattice wide BCS pairing. In higher temperature superconductors, the wave function transition (at the BCSBEC cross over [155]) to a condensate of BCS pairs way tighter [149] (e.g. on the site of super atoms [150]). At lower temperatures, electrons come and go into widely spread pairs [149].

In our multi-fold universe $U_{M F}$, folds are activated between the pairs (when the pair exists) creating an attractive effective potential in $(1 / r)$ towards the center of gravity of the pairs that is actually spread from one electron to the other by phonons (or possibly other intermediaries in higher temperature superconductors as the jury is still out on being able to model that as complex phonons, or differently). The entanglement is most probably involving these intermediaries (e.g. exchanges of entangled phonons) but the end result amounts to the same. This continuous exchange back and forth of phonons to maintain entanglement is why we believe we have more direct entanglement (not hierarchical) or at least appearing as if direct (electron to phonon/phonon to electron).

For high temperature superconductors, we believe the tighter pairs also argue for direct entanglement. Of course, this could be wrong, and attraction may only occur between electron and phonons (or whatever carries the entanglement). In general, this should probably along with attraction, and the mechanisms we described. Attraction comes and goes in waves as new electrons are paired and others leave the pairs (and as entangled phonons are exchanged). The attraction does not really reach beyond the maximum radius of the entangled BCS pairs and surrounding the superconductor as the pairs never "leave" the superconductor material to spread further. Attraction is relatively consistent towards the center of mass of the semi-conductor. In BEC, the pairs are way tighter, which means even shorter range than BCS effects but stronger attraction.

Overall, the attractions are spread all over the superconductor material as, in general, the pairs are not really identifiable; they behave as a set of similar particles.
Cross-pairs entanglement it-self depend on the entanglement mechanism. It may be associated to multi-folds, but it may also include hierarchical behaviors without additional entanglement effective potentials. The short range (limited to the superconductor and possibly its immediate neighborhood) of the gravity like effect coincidentally match some claims focused on superconductors to study gravitons found in fringe literature ${ }^{110}$.

With gravitons potentially related to multi-folds and the propagation of gravity like effects with superconductors at speeds lower than $c$, superconductors would contain massive gravitons in $U_{M F}$ and we would have a stronger attraction within High Temperature superconductors (noting of course that this is theoretical and that gravitons have not yet been formally introduced (no quantization yet) in our multi-fold universe model). [156] argues the same based on their experiments, relying on the weak gravity field approximation and associated gravitoelectromagnetic linearized approximation of $\mathbf{G R}^{111}$, as in [227, 302, 303]. Repeating the arguments of $[156,157]$, we could envisage that statistical effects in superconductors could distort (i.e., create gravity-like fluctuations) with some of the massive gravitons if the entangled pairs are made to move to the surface via rotation of the material and/or with magnetic fields because of spontaneous vacuum symmetry breaking in the solid- e.g. due to frame dragging (which can also be derived in $U_{M F}$ by working in the rotating frame). It would be analogous to the London current for electromagnetism met in superconductors that expels magnetic field and creates a London's moment [157]. This aspect is speculative and based on the idea that the folds would be massive gravitons and be sensible to such effects. [156] reports some possible observations ${ }^{112}$ of gravity (like) effects near rotating superconductors in a (strong) magnetic field.

These fluctuating attractive potentials should also contribute to the pair consistency/attraction helping combat the coulomb repulsion (in addition to the gravity also contributed as attraction per our model), albeit tiny, and other effects that may otherwise weaken the pairs; beyond what has so far been estimated: it increases the coupling both of BCS (possibly able to exist at a slightly higher temperature) and localized BEC pairs able to also form at slightly lower temperature and last till above slightly higher temperatures. But all these effects will be small and probably undetectable for ages, at least. Finally, if BCS pairs and BEC pairs are hierarchical, we believe that the entanglement between electrons of the pair and the carrier of the pairing will be direct and generate these fluctuations. Yet the analysis in this paragraph may not hold.

It would be especially interesting to see if any attractive potentials or gravity like fluctuations appear near the latest most exotics superconductors like magic-angle graphene superlattices [158] and in compressed hydrogen turned into a metal [159, 160] or twisted graphene layers as in [287]. As far as we know, such attractive forces are not modelled in any existing theories of superconductivity and other Bose Einstein condensates besides the considerations and experiments mentioned above. 


\subsection{Other Quantum Materials}

It is for future works to study the impact of other examples of quantum matter [141], BEC, superfluids, strange metals, etc. But in general, the principle is the same: whenever the material behavior is due to entanglement (direct-not hierarchical), there will appear (gravity like) attractive potentials and fluctuations between the entangled entities and within the material. The distribution of the potential depends on how entanglement is taking place and how it is distributed within the material.

\subsection{Big Bang's Primordial Soup of Quark Gluon Plasma}

The quark gluon plasma (QGP) [164] is a state of matter in quantum chromodynamics (QCD) that exists at extremely high temperature and/or density. It is believed to have existed as primordial soup associated to the Big Bang. In the chronology of the big bang, QGP characterizes the dominant state of the universe after inflation $[\underline{167}, \underline{165}]$ - assuming that inflation existed ${ }^{113}$. Evidence has been presented that it could exist in the core of massive neutron stars [495], where the effects, that we predict due to entanglement, could significantly increase the gravity effect, considering the scales and masses involved.

QGP is highly entangled and behaving like a perfect liquid and often like a BEC [161, 162]. QGP has also been reconstructed in particle accelerators $[163,164]$. As in all the other cases, entanglement creates $V_{\text {eff }}$ attractive potentials within the QGP plasma-a potential noteworthy future experimentation when studying QGP.

\subsection{Trapped ions and other types of Qubits in Quantum Computing}

Quantum computing relies on manipulating entanglement of Qubit elements or between Qubits [168]. The art of building quantum computer is the art of building robust, reliable, and efficient mechanisms to manipulate the Qubits and keep them entangled long enough, or in known or measurable states.

As the components of the Qubits are entangled, attractive potentials appear between them in $U_{M F}$. Measuring such fluctuation of gravity like forces would be a worthy endeavor. Note however that when Qubits are entangled with other Qubits, attractive potential will only appear when the entanglement is non-hierarchical as discussed before. So, if the attractive $V_{e f f}$ potential can be detected it should also be possible to detect its absence in direct entanglement and its absence (as result of force composition) in hierarchical cases. It relates also to the notion of non-observability of entanglement [87] already mentioned earlier on. While entanglement is not observable, its effect in the form of $V_{\text {eff }}$ is and will at some point be a useful feature for quantum computing if applicable to $U_{\text {real }}$. Unfortunately, today, the intensity is beyond the reach of measurements. It will remain so for probably a long time.

\section{GRAVITY}

\subsection{Gravity emergence from Entanglement \& no supra luminosity}

We have seen that in a multi-fold universe $U_{M F}$, entangled particles generate an attractive effective potential in $(1 / r)$, often isotropic (unless if associated to conditions that privilege certain directions of entanglement, which will have an additional contribution in that direction) that propagates as a wave (at the speed of the entangled particles) in $\mathcal{R}_{B G}$. It can sometimes be unlimited in range (defined by the involved entangled particles in some cases, potentially as far as $c$ allows it to go otherwise).

The gravity effective potential from one particle propagates at the speed of the virtual or entangled particles $(\leq c)$ in $\mathcal{R}_{B G}$. Macroscopically, the resulting potential is also proportional to the amount of particle presents, their masses or energies (that creates localized particles). For massive particles, it is proportional to the mass that contribute to the potential. The contribution matches the expectations of Newton law of gravity at large distances and for reasonable speeds of masses/energies and recovers average or effective Ricci curvature scalar proportional to energy or mass present in spacetime (i.e., Einstein's GR field equations). We derived them in section 4.8 .

So far, in our proposed multi-fold universe $U_{M F}$, gravity appears through the $V_{\text {eff }}$ contributions to the Lagrangian or Action (and an average effective Ricci scalar curvature + a direction capturing the contribution to the Ricci tensor). It is not the result of an omnipresent field against a static background. Instead, it is the result of the additional activated folds and their curvatures in spacetime added to the paths in $\mathcal{R}_{B G}$. For us, gravity results from these additional paths associated to all the activated folds; not from additional terms in the action or the Lagrangian; even if it is equivalent to adding such a potential $V_{\text {eff }}$ to the Lagrangian and equations of motion. Indeed, the Lagrangian addition coming from our model is:

$$
\mathcal{L}_{\text {total }}=\mathcal{L}_{S M}+\mathcal{L}_{\text {Gravity-with-MF }}+\mathcal{L}_{E P R}+\mathcal{L}_{\text {others } \ldots . . .}
$$

In equation (22), the first term is the Standard Model Lagrangian [97, 172]. The second term results from our derivation of gravity in our multi-fold universe. The third term captures the gravity like attraction resulting from entanglement. The last term captures any other effects or interactions not yet modelled in physics and coupling gravity to matter fields (e.g. Fermion spin coupling (and possibly torsion) as in [294, 290, 293, 291, 296, 25]; something that is not discussed or modeled further as part of this paper). We suspect that this last term is probably not null.

$$
\begin{aligned}
\mathcal{P} \boldsymbol{I}_{\text {total }_{S F}}=\mathcal{P} \boldsymbol{I}_{\mathcal{R}_{B G_{S F}}}\left(S_{S M}, \psi\right) \\
+\propto \int_{\Gamma_{B_{\text {active }}}} \mathcal{P} \boldsymbol{I}_{B_{\text {active }_{S F}}}(S(\gamma), \psi) \mathcal{D}_{\gamma}
\end{aligned}
$$


The second term

$$
\begin{array}{r}
\int_{\Gamma_{\mathcal{R}_{B G} \cap M^{-1} \Gamma_{\mathcal{B}_{\text {activ }}}}} \mathcal{P} \boldsymbol{I}_{M(\gamma)_{S F}} \Delta \mathcal{S}_{\mathcal{B}}(M(\gamma), \psi) \mathcal{D}_{\gamma}+ \\
\{\text { (Jacobian })=0\}
\end{array}
$$

Where $\Delta \mathcal{S}_{\mathcal{B}}(M(\gamma), \psi)$ is essentially the difference between Lagrangian/Action mapped back from folds to $\mathcal{R}_{B G}$ minus the existing contribution on $\mathcal{R}_{B G}$ and we have seen that it contributes to $\mathcal{L}_{\text {Gravity-with-MF }}$ with a $V_{\text {eff }}($ Grav $)$ (contributed by all other energy entities/masses further than an uncertainty ball and that has been able to reach a given point with $V_{\text {eff }}$ adjusted for simple relativity effects due to motion and possible massless behaviors). The Jacobian leads to zero extra contributions as the mapping is built to match momentum in $\mathcal{R}_{B G}$ to the folds.

The Path Integral formulation of quantum mechanics and field theory in a multi-fold universe $U_{M F}$ leads to Einstein GR equations (See 4.8). EPR entanglement effects are similarly modeled. What is interesting is that, at quantum scales, gravity is the result of preventing supra luminous propagators, entangled virtual particles emitted near a source of energy or particle and EPR entanglement. It is derived from quantum physics and it is not just a perturbative result or a macroscopic or statistical result as are QFT as well as $\mathbf{G R}^{114}$. This derivation results solely from the rules (folds and nothing supra luminous) of $U_{M F}$. It is not the result of a priori using, adapting or extending Hilbert-Einstein action as is done in QFT gravity, superstrings and theories like LQG (the whole family of variations as for examples reviewed in $[243]^{115}$ ) that all start from Hilbert-Einstein action. If you start from the Hilbert-Einstein action; that it be in a background dependent or independent case; of course, you will recover one way or another Einstein's GR field equations, and of course you will recover an area law (for horizons - see later ${ }^{116}$, as well as spin-2 gravitons!).

It is also to be clear that the GR field equations and $V_{\text {eff }}$ are complemented by $V_{\text {eff }}$ (MassiveVP) from the massive virtual particle contribution that amount to increasing progressively the coupling constant of gravity as the distance from the source is within the range of more and more massive virtual particles.

Similarly, $\mathcal{L}_{\text {Entanglement }}$ is proportional to relativistic $V_{e f f}(E P R)$ defined as the contributions from $x_{1}^{\mu}$ and $x_{2}^{\mu}$ of the terms as in equation (20). Depending on what particles are entangled, effects propagate a $c$, or slower. This is not gravity per se. However, it may have large-scale effects that look like gravity as we will see later. At this stage we know:

- For classical, semi classical and quantum scales, we have already discussed that the multi-fold mechanism is:

- spin-2 invariant, which hints at spin-2 gravitons if/when our framework is quantized but living in $\mathbf{A d S}(5)$.

-violating $\mathrm{T}$ symmetry (time-reversal) and other combinations of $\mathrm{C}, \mathrm{P}$ and $\mathrm{T}$ symmetries (see 4.5).
- The approach is background independent and as such it does not suffer of problems related to self-interactions (matters add contributions to effective potentials and effective curvature and multi-folds or graviton live outside spacetime).

- Frame dragging, as described in gravitoelectromagnetism [227, $\underline{302}, \underline{303}]$ and mentioned in section 6.1, as well as linear frame dragging, is hinted and explained from the multi-fold mechanism for virtual particles. Indeed, virtual particles emitted by a rotating solid contribute folds $V_{\text {eff }}$; but on a straight line, the higher $\mathrm{r}$ items come from particles in the direction of rotation while the smaller $r$ contributions come from the others direction. They bring a contribution tangent in the direction of rotation, close to the rotating item. It is illustrated in Figure 10. A similar effect of linear frame dragging also exists; we discussed ideas of it with the discussion on gravity from a massless particle.

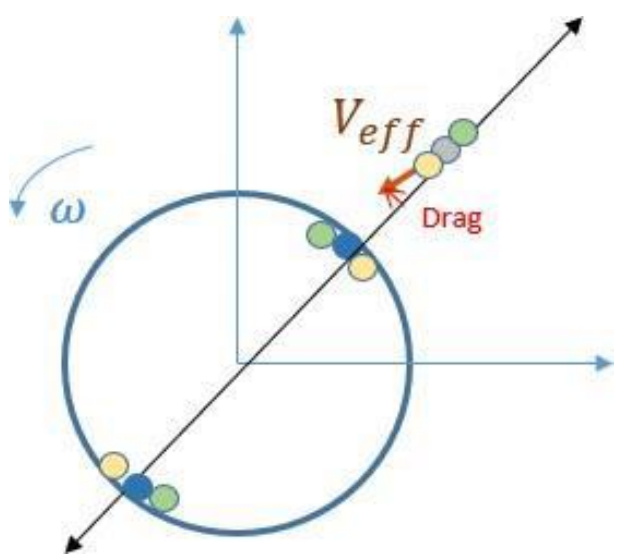

Figure 10: In the rotating frame the integration from $+\infty$, the virtual particles contribute attraction that has a tangential component in an external inertial frame. The particles contributing to a line are symbolically shown. The contribution of the particle coming from the direction opposite to the rotation dominates. Close enough to the rotating body, it brings a $V_{e f f}$ contribution in the direction of rotation.

- Multi-folds are curved spacetime, which a priori could lead to problems as, in a curved spacetime, the number of particles and the ground state depend on the referential and would be disagreed upon by different observers. However, with respect to the center of mass referential the folds are spherically symmetric. They produce no time-dependencies of the metrics, no time-space cross terms [177] and any time dependency (i.e., which fold to consider) is not affecting the model because it amounts to fold shuffling or shifting of their time label in $B_{\text {activ }}\left(x^{\mu}\right)$ for virtual particles. For EPR entanglement, there is a time dependency of the folds and their order. All this implies no spacetime cross terms modifying the number of particles in the folds because of the curvature [171] for surrounding virtual particle effects. If/when 
quantized, we can expect massive bosons to appear in the presence of EPR entanglement of massive particles.

- As we proposed earlier, no interaction is taking place in the folds. However, spins may couple with the fold curvature; with the option of a torsion less or torsion impact within the fold $[\underline{302}, \underline{294}, \underline{304}, \underline{290}, \underline{293}, \underline{291}, \underline{296}$, 25]. For this paper, in the folds, we assume only usage of spin connection without torsion.

- The Path Integrals are (locally) Lorentz covariant methods. Locally, all computations in $\mathcal{R}_{B G}$, and in folds are covariant, as are the events and fold kinematics or dynamics: everything is relative. However, the "simultaneity" of the events may not be Lorentz invariants outside the frames we considered. In general, in the presence of matter (real, virtual or vacuum), there is a break of the global Lorentz symmetry that announces the massless or massive graviton if / when the theory is quantized. When $\mathcal{R}_{B G}$ is flat, global symmetries can exist in the absence of matter or energy. When matter or energy is added, the folds contribute curvatures in such a way that gauge symmetries or diffeomorphism are probably no more globally invariants, in agreement with [361, 362]. It also announces a massless graviton associated to virtual particles induced gravity (if or when quantized). Traditionally the viewpoint on the origin of massless gravitons is not agreed upon. See [ $\underline{305}, \underline{306}]$ for some points of view when quantizing GR. The contributions of massive virtual particles modify at very short scales (reachable by these virtual particles) by adding their (gradual) contributions. The same reasoning as presented above applies and we are predicting that a "massive" very short scale version of GR also appears along with massive gravitons, if and when quantized ${ }^{117}$. EPR multi-fold mechanisms are also associated to massive or massless gravity- like behaviors but anisotropic (unless if produced by isotropic sources of entanglement). They are an addition to gravity that also involves massless or massive gravitation behavior if or when quantized.

- The absolute no supra luminous requirement may not break the global ground state invariance under Lorentz transformations. In addition, different observers will see different number of particles and not agree on the vacuum when matter or energy is present and generically distributed (see [171]) so that metric of the resulting curved space approximation of the effects of all their effective relativistic potentials $V_{\text {eff }}$ on $\mathcal{R}_{B G}$ is time dependent (which happens if distributions are not static) or introduces time-space terms.

- By construction, the approach and model proposed is not perturbative: the theory of $U_{M F}$ is fundamentally a microscopic ${ }^{118}$ as well as a macroscopic model and semi classical considerations can go down to very small scales when dealing with the rest of QFT.

The formulation of equation (24) strongly indicates that gravity effect Actions, Hamiltonians and Lagrangian densities depends only on the relativistic $V_{\text {eff }}$ distributions. These are functions of the Ricci curvature scalar of the folds and lead to Einstein Equation, when keeping out entanglements between EPR entangled particles as well as massive virtual particles gravity contributions. The contributions to the Lagrangian (densities) is a sum (integral) of relativistic $V_{\text {eff }}$ potentials (densities) which is a distribution of Ricci scalar curvature contributions from all the activated folds. We showed in section 4.8 that it provides a new effective Ricci curvature scalar and tensor and that the Hilbert-Einstein action reflects actually that scalar. Another consequence of the reasoning and construction of the action is that Hilbert-Einstein action is the correct action and not a first order approximation in $R$ as sometimes proposed [23, $\underline{300}, \underline{298}, \underline{299}, \underline{25}, \underline{308}, \underline{295}, \underline{294}, \underline{297]}$ (and many more proposals exist and keep on coming). Earlier, we also showed a possible additional microscopic torsion (that may or may not exist). When considered, it removes all gravity singularities from $U_{M F}$ and its cosmology and supports Big Bounce solutions. As a side note, it is worth mentioning that, in a multi-fold universe $U_{M F}$, the proposed lab experiment to validate gravity as a quantum force [174, 175] may probably not answer or confirm what they expect, and for other reasons than what is argued in the debate unfolding in [176]. Indeed, we show that gravity results from enabling entanglement. So, detecting entanglement as proposed may be the result of gravity or other entanglement enablement. But yes, in our view, it could demonstrate the quantum nature of gravity. Let us now discuss selected other views and works on gravity that relate to our work.

\subsection{MOND}

In a multi-fold universe $U_{M F}$, our approach and derivation of gravity is not a MOND [178], even with the gradual massive gravity contribution (mostly at very small scale) and gravity like (but anisotropic) contributions from EPR entanglements. We do not dispute that other contributions to gravity might exist, but they would not result from our model; unless something special happens at the level of entanglement across the universe; something that indeed may happen with dark matter as we will discuss later. A gravity weakening due to massive gravity does not appear at very large scale, and so it is not a weakening of Newton gravity at very large scale as is the concept and motivation for what is usually classified as MOND.

So, in a multi-fold universe $U_{M F}$, gravity at large scale follows strictly GR, and when suitable, Newton gravity. However, we know that we have also introduced an attractive potential resulting from entanglement. This component adds to traditional gravity. We do not see it as modifying gravity but rather adding a different contribution to nature. At long range it may explain dark matter as we will discuss later.

\subsection{Entropic emergence of gravity}

We already mentioned emergent theories. Our whole motivation for revisiting and publishing our multi-fold universe model came from reading the widely publicized 
[11] concepts of entropic emergence of gravity, and how it would explain gravity, dark matter and dark energy as a statistical (i.e., entropic) effect of entanglement at different scales [14]. From the beginning we thought that the concept resonated with our model, yet the models are different. Entropic emergent gravity describes gravity as an entropic force. i.e., subject to quantum-level disorder, and therefore no more as a fundamental interaction. Instead, gravity would result from the quantum entanglement of small bits of spacetime information. As such, it would also follow the (second) law(s) of thermodynamics. It is to be noted that in our view, the inspiration and origin really comes from [18], where Einstein's field equations were derived by combining general thermodynamic considerations with the equivalence principle.

Building on works following [18], [14] also provides a framework to introduce and explain MOND where gravity would weaken at large scale with the force (not potential) decreasing linearly from the distance of a mass beyond a certain scale. [10] also tries to derive Newton's laws and Gravity (Newtonian and General Relativity) by showing that if we have Einstein equivalence principle, then it all happens because inertia is an entropic force.

Then, $[14\rceil$ models the entropy of short range (microscopic) resulting from QFT vacuum field entanglement and large scale (macroscopic) entanglement across space-time. It relies on area properties valid in Anti de Sitter spaces that it transposes with AdS/CFT correspondence and holographic principal arguments, to a de Sitter universe. It a questionable step, and in fact questioned for example in [309]. The holographic principle for QFT gives area-based entropy contributions that would be overcome by volume based large scale contributions to entropy. With gravity derived as describing the change in entanglement caused by matter (microscopic) and spacetime to encode that information as well as describing large scale bulk entanglement that brings in a more elastic behavior, Verlinde derives a MOND and does not require dark matter to account for the astronomic observation challenges that led to the introduction of dark matter [310]. Unfortunately, in our reading of [14] and previous works, we had to make several leaps of fates [309]. As expected, considering the approach, some observations [181] and models [180, 179] seem to invalidate some of the assumptions and predictions. For example, [179] questions the suitability of this approach and the value to attach to its derivation of gravity.

Considering the keywords ${ }^{119}$ similarities to our approach, it is probably important to understand the relationship with our model. In a multi-fold universe $U_{M F}$, we do not propose that entanglement generates an entropic force that encodes entanglement information. Instead, gravity results from multi-fold activation due to entanglement. These multi-folds create effective attractive potentials by contributing additional effective curvature to spacetime, as perceived by other particles. These effects, when looked at larger scales involving many particles or field/energy densities, reconstruct Einstein and Newtown gravity at the appropriate classical or semi classical scales. So, gravity emerges from the behavior of spacetime and GR emerges from statistically large models of particles; but gravity is not an entropic force (à la [10]) nor a MOND (à la [14]): gravity exists at the microscopic level and results from the activation of bundles of folds. Of course, EPR entanglement also contributes. As it results from EPR entanglement, gravity is as deeply quantum in nature and fundamental as an interaction. It results even at the microscopic individual level from fundamental quantum behavior of the multi-fold universe. Yes, it is expected to follow the laws of thermodynamics as long as they are adapted to the peculiarities created by the multi-folds, and therefore GR can be derived from thermodynamics per [18]. A full thermodynamics model of our multi-fold universe is of course of interest and possible. We will address some of these points soon.

\subsection{Gauss theorem, Area Laws and Holographic Principles in $U_{M F}$}

Let us see how some gravity specific theorems evolve or apply in $U_{M F}$. The spin-2 like rotational axial symmetry of the bundle of folds $B_{\text {activ }}\left(x^{\mu}\right)$ around a particle is a "sticklike" symmetry. It anticipates spin 2 massless and massive particles (if and when quantized). For now, let us assume only the presence of massless gravity (no EPR particle entanglements ${ }^{120}$ and no massive graviton contributions), somewhere high enough above Planck scale. We will also perform our analysis and proofs with the approach where all curvatures come from multi-folds and therefore initial conditions are a flat $\mathcal{R}_{B G}{ }^{121}$.

Let us first consider a closed surface $\Sigma$ in space in $U_{M F}$. For any two points $x_{1}^{a}$ and $x_{2}^{a}$ of $\Sigma$. At $x_{1}^{a}$, one cannot distinguish the contributions to a point $x^{a}$ within the surface from within $\left[x_{1}^{a}, x_{2}^{a}\right]$. This is because of the way that folds contribute (i.e., at $x_{1}^{a}: 1 / r^{2}$ from $r$ to $\left.+\infty\right)$.

The same applies at $x_{2}^{a}$. It is true no matter how many times we multiply it by mass units of the points insides. So, any surface $\Sigma^{\prime}$ within the volume of $\Sigma$ and all the entities in the volume in between them brought to $\Sigma^{\prime}$ will appear the same on $\Sigma$ and beyond. If a mass/energy is within the surface, the flow across $\Sigma^{\prime}$ rather reflects it. This is a way of recovering a version of Gauss divergence theorem ${ }^{122}$ for gravity $[\underline{182}$, 183] in $U_{M F}$. As a result, we also have a holographic principle that maps what happens in a volume to the closed surfaces encircling it (mapped bijectively via a straight line in $U$ not modelling statistical curvatures). The same applies for massless sources, but requires adding the light sheets built on $\Sigma$. It is that model that also behind the covariant entropy bound as in figure 3 of [518].

Let us now focus on black holes. Consider a static case. We already know that per the approach proposed ${ }^{123}$ that gravitational singularities do not exactly exist in $U_{M F}$. They are really resulting out of the approximations:

- Continuous model of spacetime instead of discrete as discussed later.

- Statistical model instead of individual particle representations behind GR and QFT.

- No model of torsion at classical/macroscopic scales. 
It is worth wondering why a black hole horizon would still appear in $U_{M F}$, as each entity contributes bundles of folds. They pile up the contributions at a given point. and these contributions are equivalent to concentrating the mass of all the contributions at the center (not necessarily at a point but in an uncertainty region of Planck's scale. We realize rapidly that we can again reach situations where the escape velocity of any entity would have to be larger than $c$. That is forbidden in $U_{M F}$. So, we still have a horizon.

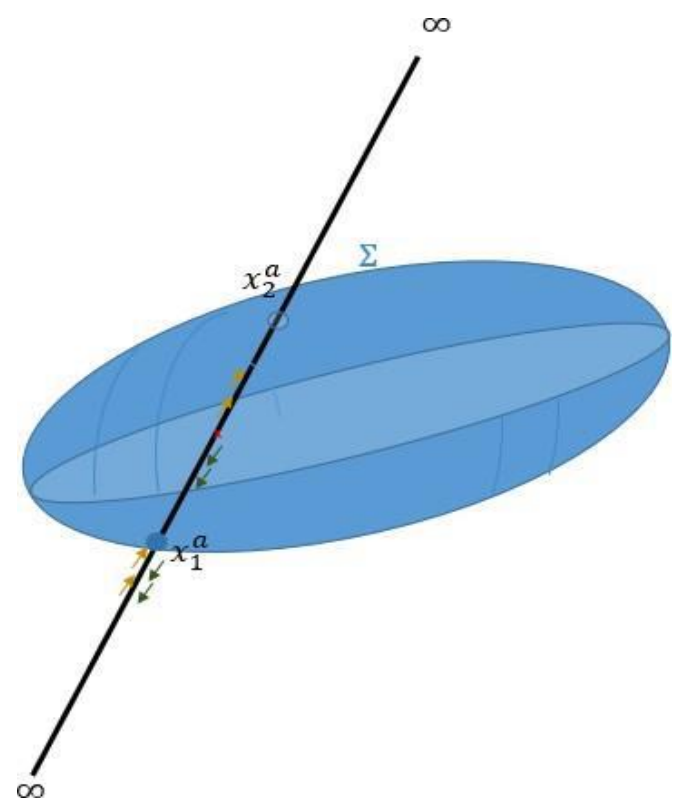

Figure 11: If a contributing point is moved between $\left[x_{1}^{a}, x_{1}^{a}\right]$, the gain or loss of contributing folds felt by $x_{1}^{a}$ is compensate by the loss or gain coming from change on $x_{2}$ side. As a result, points can be moved to a more internal surface $\Sigma^{\prime}$ the effect felt on $\Sigma$. When the surface is not static, or we consider massless sources, we need to revert to the lights sheets built on $\Sigma$, as in [518].

This horizon matches the approximations of GR. For example, the escape velocity at $\mathrm{c}$ for an object of mass $M$ is reached at a radius that matches Schwarzschild radius: $r_{e s c_{c}}=\frac{2 G M}{c^{2}}$ (replace $c$ by $v_{e s c}$ and we have the escape velocity in Newtonian gravity) ${ }^{124}$.

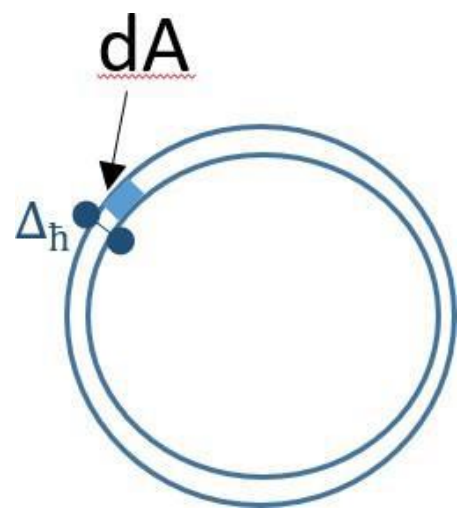

Figure 12: All the gravity effects of the black hole are captured in a shell of constant depth and a surface $A$. These represent all the gravity or spacetime degrees of freedom of the black hole as they (and only these points) generate what is perceived outside the black hole. The Gauss theorem works with $\Sigma$ (no light sheets needed) as all trapped particles (real or virtual) behave massively when trying to exit: the massless multifold pattern can be ignored.

At the horizon, particles cannot emit anything towards the outside of the black hole as it would require a speed faster the $c$, which is forbidden in $U_{M F}$. But with the uncertainty principle, a border of length determined by the uncertainty 
principle appears where particles can be emitted. As a result, the black hole is perceived outside its horizon is due entirely and solely to that shell of surface A and depth $\delta_{r+\hbar}$ with $\delta_{r+\hbar}$ a constant reflecting the uncertainty principle. As a result, the gravity effect of the black hole is entirely produced by this uncertainty vacuous spacetime in the shell. And the gravity effect of the black hole can be also seen as equivalent by putting all its entities on its horizon per our Gauss theorem. Therefore, all the degrees of freedom of the black holes are proportional to that surface $\propto A \delta_{r+\hbar}$ which reproduces its gravitational effect. We recover in $U_{M F}$, the Area law of entropy for black holes [311, 312, 313, 18, 314]. It is also known as the entropy law of black holes.

Because internal folds are blocked at the horizon (as no (virtual) particle escapes this way and therefore no fold can go beyond), the gravity effect from the inside is analogously perceived outside as the entanglement of the horizon (inside $\left.\delta_{r-\hbar}\right)$ with virtual particles in the bulk inside the black hole. One might try to interpret the entropy of the black hole as the maximum density of massive ${ }^{125}$ gravitons coupled with their virtual particles that can be "stored" at the surface (where they can live forever as time stops for all the particles there) and hence an area dependency make sense, itself being dependent on the total energy or mass of the black hole. When it comes to boson carrying charges (e.g. electromagnetic, color, etc.), they similarly aggregate there. It is a stationary value for a given total mass of the black hole and lives in the spacetime of the shell resulting from quantum uncertainty. There it adds to the vacuum to produce gravity effects proportional in total to the mass of the black hole. External folds from external particles have a similar fate at the horizon, and external virtual particles with their folds (and other carrier bosons) are entangled with virtual bulk particles on the outside of the black hole. They make the black hole aware of the (negligible) gravity from these external particles and the external world that they represent. So, regarding the widely held view that the entropy of a black hole is the result of entanglement between the inside and the outside (e.g., [186]), we agree but with some caution about such a view in $U_{M F}$. Indeed, there is entanglement between inside and outside of the black hole, but it is due to the described quantum fluctuations of the horizon.

There is a layer of internally entangled particles, who generate folds, and because of the uncertainty fluctuations of the horizon; some of them are allowed to escape a la Hawking radiations.

Hawking and others have tried to extend the law to other horizons $[315, \underline{318}, \underline{18}, \underline{316}, \underline{314}, \underline{317]}$ or even more generically between spacetime volume and area and use them as basic constructs of spacetime. It is in fact a fundamental result of GR where the Hilbert Action amounts to extremize areas (i.e., make them invariants) to the dynamics of spacetime (it results from the geometrical interpretation of the Ricci curvature scalar $\mathrm{R}$ as the ratio of the area of small spheres around points on the manifold from and to the same area of small spheres around points on flat space $[\underline{364}, \underline{363}]$.

In fact, Regge calculus shows that Hilbert Einstein equation discretized on a Regge lattice actually express the Action as areas (or deficit angles) to extremize [23]. We can now show that in $U_{M F}$, for any change of the area of a closed surface $\Sigma=\partial V$, the entropy change is proportional to the area change. It can be shown by repeating the argument above for black holes but without black holes and push all the matter inside $V$ to $\Sigma$.

All the effect of gravity outside $\Sigma$ is implemented by particles on $\Sigma$. This indicates that the degrees of freedom and so the entropy are proportional to the area (and $\propto A \delta_{r+\hbar}$ will again appear as with the uncertainty mode degrees of freedom are involved.).

Armed with these results, we can apply the reasoning of Jacobson in [18] to derive Einstein's GR equations, this time starting from the $d S \propto d A$ relationship applied along causal horizons; assuming an ultraviolet cut-off at the Planck scale for the field entropy. The considerations above are based on well-known works; nothing exceptional. In fact, it was expected from the moment that we showed that $V_{\text {eff }}$ of gravity was attractive in $(1 / r)$ as a result of the activated bundle of folds. Yet it is interesting to see that the fold mechanisms ${ }^{126}$ along with the quantum physics formalism of Path Integrals, results into:

- $(1 / r)$ attractive potential densities proportionality to masses working from classical scales down to quantum scales.

- Einstein's field GR equations based on the mass or energy distribution, at classical and semi classical scales. We can also build an effective curvature (scalar and tensor) field as in our derivation of $\mathbf{G R}$.

- Entropy area law for any closed surface, with a dependency on an uncertainty constant; not just limited to black hole horizons but also at the level of spacetime (and more generic casual horizons). This is a generic result that seems to still be a conjecture in $U_{\text {real }}$.

- Thermodynamics derivation of Einstein's field GR equations by applying the area laws to causal horizons.

- Spin-2 like symmetry for Gravity.

- Generic holographic principles formulated rather like a consequence of the Gauss divergence theorem for gravity, and the baton symmetry within $U_{M F}$ (in $\mathcal{R}_{B G}$ ) as well as a correspondence between $U_{M F}$ and $\mathbf{A d S}(5)$ through the multi-folds with the mapping $\mathrm{M}$, whereby multi-folds/gravitons/gravity live in $\mathbf{A d S}(5)$ and impact $\mathcal{R}_{B G}$ via attractive $V_{\text {eff }}$ (and apparent effective curvature effects). The latter is really what becomes our version (for $U_{M F}$ ) and hints why there would be such a correspondence in superstring theory.

In our analysis above, we also assumed scales larger than not only Planck length, but also the range of interactions with massive virtual carriers (e.g. weak interaction, gluon and meson driven strong interactions $)^{127}$. Below these ranges $\left(\sim 10^{-15} \mathrm{~m}\right)$, effects of massive virtual carriers of gravity can also appear in the form of additional contributions to the attraction (also in $1 / r$ ). The short-range aspect results into 
consequences like those mentioned below for EPR entanglement. From now on, we will assume that gravity has a short-range component due to massive gravitons. This is considered as an entanglement situation analogous to EPR entanglement but between massive virtual carriers. This has consequences mostly in the sense that these entanglements are present everywhere at small scales but scales that are significantly bigger than Planck scale. So, in $U_{M F}$, at Planck scale massive gravitons carriers and systematic presence of EPR entanglement even when no real particles are EPR entangled is another key difference with what is currently considered by all attempts to model quantum gravity (in $U_{\text {real }}$ and in other exotic spaces with more dimensions or AdS universes. With short range (massive) gravity, the Gauss theorem is no more satisfied nor is it with anisotropic or individual EPR entanglement, except when conspiring the surface with its light sheets mentioned earlier. Holographic correspondence remains with respect to $\mathbf{A d S}(5)$ by construction of the multi-fold mechanisms. All the above was computed for the gravity and entanglement contributions. Other (quantum fields) can contribute through their carrier particles as mentioned earlier. In the presence of fields, it has been showed that the entropy contribution to a black hole is also finite and proportional to the area of the black hole horizon [185] [232] $]^{128}$. In $U_{M F}$, these carriers accumulate at the horizon of black holes, behave as massive, and result into the famous black holes hairs for charges (electromagnetic, colors, isospin and anything else that is conserved. Confinement of course also applies for colors and hides color charges.). It also assumes the same Planck scale cut-off. Entropy statements as above are not extensible, generically, to other horizons or closed surfaces; but it holds for causal horizons (and surfaces with light sheets); in particular keeping the Thermodynamics of spacetime correct in the presence of fields and allowing us to recover also Einstein's field equations in the presence of quantum fields in spacetime. The shell of uncertainty used above is actually our version of the micro hairs to the black hole [378] that modernizes the no hair theorem: besides Mass, Charge and angular momentum, black holes can have local microscopic fields and properties related to the virtual particles (and hence carriers) that are involved. It could also be seen as tunneling. It means including in the microscopic region of thickness $\delta_{r+\hbar}$ strong and weak properties and virtual carriers.

Bekenstein's values for the black hole entropy is a total entropy and it considers all these contributions. In fact, all the points above have to hold for black holes and any other closed surface which is a causal horizon and/or define an autonomous region (e.g. with light sheets). Entropy is either computed with gravity or via QFT and their entanglement, one or the other. So non-gravitational degrees of freedoms coming from quantum field respect the theorems for such surfaces. The caveats about entanglement and massive gravity still hold, and require the light sheets. Alternatively, without it, massive gravity contributes now to the micro soft hairs, as do any EPR entanglement within the horizon. The lower the entropy, consistent with the notion of maximum black hole entropy or entropy inequality. Earlier in the sections we indicated that the analysis assumed that no entanglement between real particles was considered to derive the area/horizon theorems in $U_{M F}$. In the presence of entanglement between real particle, the theorems in general do not hold in $U_{M F}$ the same way and require the light sheets. For black holes all particles who try escape are hindered, and so massive, and we can avoid the light sheets. Alternatively, one could argue that massive gravity contributes to the micro soft hairs, at the horizon.

- The Gauss divergence theorem for gravity holds only when the closed surfaces (without considering the light sheet) considered are either smaller that the distance between the EPR entangled particles or surround them all enough further away It is fair to say that in the presence of EPR entanglement, the theorem does not hold in general any more in $U_{M F}$ and in any case the source term would have to now incorporate a measure of entanglement, function of the entanglement entropy. For massive gravity, at small scale, it is probably impossible to find, in general, a surface that fits these requirements as new contributions appear as distance decrease; so, it does not hold in general without introducing the light sheets. Of course, the theorem still holds for massless gravity, also if light sheets are considered.

- For more discussions and references on Area laws and entanglement, we refer the reader to [491]; entanglements do respect Area laws also but not always, as we saw here; and it is complex.

- Massive gravity microscopic contributions are similarly handled, and they increase entropy ${ }^{129}$. As the effects at the horizon also come only from a shell near the horizon, it remains an area law.

- The entropy computed for black holes includes all fields and entanglement that it expresses equivalently as gravity or as entanglement: the area law for black holes is therefore the actual entropy in presence of entanglement behind the horizon. The actual entropy of a black hole is $S_{B H}=S_{\text {Grav }_{B H}}-S_{\text {Entang }}(\mathbf{Q F T}) \quad$ where $\quad S_{B H}$ is Bekenstein's entropy [311] and $S_{\text {Entang }}$ can be well estimate by Von Neumann's entanglement entropy. For a way to understand that, see an upcoming section: spacetime are microscopic black holes as are particles and therefore field, but now excited. The entropy of the particles are reflected into entropy of the entanglement based on von Neumann or alternatively captured in the entropy changes of spacetime. One can use one of them. They are not additive, but one can substitute for the other. A consequence is that the entropy of gravity for the black hole or Bekenstein-Hawking entropy can also be also as coming entirely from the QFT fields [232]. Entropy of gravity comes from $\mathbf{A d S}(5)$ consistent with RyuTakayanagi conjecture, with gravity entropy in $\mathbf{A d S}(5)$ [317].

- Entanglement may not follow an area law at very small scales, i.e. for high energy contributions, or excitations of the black hole [186], contrary to [185]. Also note that 
EPR entanglement cannot happen across causal horizons. We will revisit later.

- EPR entanglement, involves irreversible processes, or at least away from equilibrium (e.g. fold dynamics/activation and disentanglement with fold deactivation), including for gravity built on these mechanisms. It produces a direction to the arrow of time and may after all give reason to Eddington [379]. Indeed, we have now shown how fundamental gravity and entanglement are in $U_{M F}$ and as such they are the only irreversible fundamental interactions so far.

- At, Planck scale, these laws will change and may have to be adapted; in particular when it comes to Entropy estimation. Some impacts are discussed in the upcoming sections.

\subsection{Microscopic (quasi) black holes in the neighborhood of particles}

In previous sections, we identified that the bundle of activated folds by entangled particles and especially surrounding physical and virtual particles create an attractive potential in $(1 / r)^{130}$, mostly isotropic unless if a preferred momentum direction has to be explicitly handled. The particles appear almost like a microscopic quasi black hole due to the local mapping to the folds; at least up an uncertainty length ${ }^{131}$.

For the mass of a particle, the horizon with a radius roughly given by Schwarzschild radius is smaller than the uncertainty ball, and so it is not visible at all in conventional classical or quantum physics. Every entity that we understand in conventional physics (classical and quantum) interacts for all purpose away from the microscopic black holes in a (combination) of Schwarzschild spacetimes ${ }^{132}$.

The black holes are (effectively) carved in $R_{B G}$ (by the phenomena of creating an "effective" potential (or curvature) out of the fold curvatures. They surround every particle. These considerations open the possibility that the microscopic black holes hint in fact at the mechanisms through which folds are activated/created by carving the fold into the tangent dual $\mathbf{A d S}$ (5) space that we mentioned earlier (something aligned with [190]).

Macroscopically, similar concepts have already been met in general relativity with the Einstein Rosen bridges [89] and the wormhole solutions that are associated to it. See, for example, [오, 332, 90], including the ER = EPR conjecture [86] already discussed. It is worth mentioning also explicitly the work of $[184, \underline{32}]$, which also postulated associating particles (i.e., with masses and charges) to microscopic black holes.

Black holes have been modeled as quantum objects $[\underline{382}$, $\underline{386}$ ], and particles have been modeled as black hole [ $\underline{384}$, $\underline{385}, \underline{383}$ ]; even if some issues exist with the horizon dimensions and/or the possibility of naked singularities $[387]^{133}$. This will be addressed in future papers, that are, or will be, available at [513]. Also, it has been shown in particular that a black hole electron would follow Dirac's equations. We will actually rely on some of these concepts in the upcoming spacetime reconstruction section 9.6.

$\mathbf{E R}=\mathbf{E P R}$ appears more like a discussion of entangled black holes. $\mathbf{E R}=\mathbf{E P R}$ is essentially analyzed in AdS and mapped by holography to CFT at the AdS boundary. Yet, its immediate generalization, or implementation would be microscopic black holes around any particles that are entangled, and interact via wormholes.

See $[188]$ for example. But these works are still more driven by the equivalence of different mathematical machineries (e.g. Holographic duality and AdS) than pursuing models of entanglement, gravity or spacetime. It is also important to remember that the folds activated in our multi-fold universe do not have a priori to be solutions to Einstein GR field equations (as they coexist outside $\mathcal{R}_{B G}$ ).

None of these works show that EPR generates gravity. By the way, our paper can also be seen as concretizing something like $\mathbf{G R}=\mathbf{Q M}$. From our point of view,[49] almost had the right ideas before drifting into holography and AdS. Anyway, at this stage we have established that every particle has a $\mathbf{A d S}(5)$ tangent dual surrounding it, and that. In $\mathcal{R}_{B G}$, particles appears as a microscopic quasi ${ }^{134}$ black hole, able to carry all quantum properties (mass, charges (electromagnetic and others), momentum as hairs/microscopic hairs).

\section{SEMI CLASSICAL STANDARD MODEL: "ADDING GRAVITY" RATHER THAN "GOING BEYOND THE SM": SMG}

In this section, we will discuss some interesting semi classical consequences of our model so far, in terms of particles and the Standard Model [131].

The outcome can be seen either as another set of validation or ways to validate some of our predictions ${ }^{135}$. Alternatively, this section illustrates how adding gravity to the Standard Model (SM) may actually resolve a few open issues in Physics. Yet the presence of massive gravity at very small scales is a game changer versus the latter kind of considerations: the effects involved in particle interactions can be significant, and, as discussed later, gravity may not be the weakest (i.e., to the point of being negligible) interaction at some of these small scales.

In general, we assume that equation (22) describes the extension of the standard model with our approach (gravity and EPR entanglement), with the last term set to zero. Note that to a large extent the resolutions hinted, and proposed in this section result from adding gravity (as a potential in $(1 / r)$, and therefore not necessarily negligible when the particles interact, or overlap ${ }^{136}$ as is the case for example with the mass acquisition with the Higgs, QCD chirality breaking and Quark-Gluon Condensate interactions.

So, the proposed resolutions can be combined with other theories similarly adding gravity (or entanglement effects) to the Standard Model. Because of the massive gravity component at very small scales, we expect that gravity contributions are not always negligible in, and at the scales of, the Standard Model, contrary to conventional thinking. We will further motivate in section 9.8, and following ones. 
The SM with gravity effect non-negligible at its scales is denoted by $S M_{G}$.

\subsection{Mass generation, gravity and the equivalence principle?}

In the standard model, there are a few ways to generate mass based on (all these mechanisms contribute to the rest mass of matter):

- Electroweak and Higgs mechanism, that provides mass to (charged) lepton137, by interaction with Higgs boson, while electroweak carrier bosons mass is due to the associated spontaneous symmetry breaking mechanism.

- The electroweak effects that give mass to the quarks.

- QCD vacuum/Quark Gluon Condensate mass generation for quark composites like protons and neutrons, and/or chirality symmetry breaking. This is in fact the main contributor to the mass of (atomic) matter/nuclei.

Our goal is, by no means, to review or add any to these mechanisms. Entry points to the topic can be found for example in [395, 280, 281] and [359] for an inspiring pedagogical approach. In general, the mass of charged leptons and quarks [396, 395], through chirality flips, are proportional to how strongly they interact with Higgs Boson and flip chirality in the process. The mass within composites of quarks adds the much stronger contribution of how the quarks interact with the sea of quarks and gluons present in the QCD vacuum or Quark Gluon condensate that surround any quark in the composite $[397,398] . V_{e f f}$ and the mass terms generated by these different mechanisms appear each time in similar ways ${ }^{138}$ :

$$
\begin{aligned}
& \mathcal{L}_{\text {int }} \propto C_{1}(\text { vertex }) \\
& +m_{\text {acquired }}\left(1+\frac{V_{\text {eff }}}{m_{\text {acquired }}}\right)\left(C_{2}(\text { lepton } / \text { quark })+c c\right)
\end{aligned}
$$

Where the $C_{1}()$ designates the vertex contribution that represents the interaction with the Higgs/QCD Vacuum and/or Quark Gluon condensate with chirality flip and $C_{2}($ ) represents the contributions of the right-handed + lefthanded leptons or quarks. In all these cases, the resulting mass term that appears in the Lagrangian is also the term that appears in the attractive potential $V_{\text {eff }}$ (21), now simply added to the Lagrangian. It automatically implies the equivalence principle ${ }^{139}$ from the multifold mechanisms in $U_{M F}$; instead of having to follow the reasoning in [399] to arrive to a similar conclusion. Equation (25) has potentially significant implications.

\subsection{The Strong CP problem and gravity}

The strong CP problem is well summarized in [324]. Essentially, QCD predicts CP violations by the strong interactions. Such violations have never been observed. [324] reviews possible ways to explain this situation. Among those, it is shown that if the mass of the up quark is zero, then contributions that produce $\mathrm{CP}$ violations disappear.

If we consider equation (25), the contributions attributed to the mass of the up quark can now be seen as attributed to a term that also includes the contributions of $V_{e f f}$, therefore allowing the up-quark mass to be zero or very close to it as an (almost/) natural symmetry; thereby, being able to resolve the problem.

\subsection{Stability of the Electroweak vacuum}

The same approach can also apply to the problem of stability of the Electroweak vacuum. Following Coleman's work $[401,402]$, it is known that the vacuum ground state may actually be a false vacuum, in the sense that a lower energy state may exist behind a barrier of potential. While that is classically not an issue, in quantum when it is the case, there is a non-negligible probability that the lower energy state will be attained at some point.

It has been considered that this situation may be met in the case of the Higgs field, and the Electroweak vacuum. The instability, if it were to exist, would be expected to result into formation of a "bubble of nothing" in spacetime; that would eventually destroy everything in the universe [403]. While the probability would be low enough that this should not happen anytime soon, it is certainly a scenario of concern. Since the Higgs boson has been discovered, and its properties studied, the stability of the electroweak vacuum has been probed. It turns out that the observed mass of the Higgs with respect to the mass of the particles that interact the most with it (i.e., the most massive particles of the SM: the top quark and the $\mathrm{Z}$ boson), puts the Higgs mass at the edge of instability [404].

The analysis above again replaces the mass of the top quark with the additional contribution of $V_{\text {eff }}$. It amounts to shifting the Higgs mass further away from the edge of instability (towards no existence of a lower level of vacuum), and, therefore, would stabilize the Electroweak vacuum (not lower minima would exist), and avoid having to explain why the Higgs boson is just at the edge, and worry about this "bubble of nothing".

\subsection{Gravity and neutrinos mysteries}

In the same vein, adding gravity to the standard model may contribute to the understanding, or the set of options, to address some of the mysteries associated to neutrinos like their mass, and mass generation mechanism [400], or what happens to the right-handed neutrinos (and left-handed anti neutrinos). Our proposal is simply to add gravity to the Standard Model, and observe that gravity can change the chirality of massless neutrinos $[405]^{140}$, or the orientation of spacetime above the energy scale of the electroweak symmetry breaking.

So, one can add these mechanisms and oscillations to the flavor and mass oscillations: in the presence of gravity, lefthanded neutrinos (massless) can, in flight, transform into right-handed neutrinos and vice versa $[405]^{141}$. Doing so, neutrinos can acquire mass via the Higgs mechanism. Once 
they have masses, flavor and mass oscillations take place as currently understood; including the fact that only left-handed neutrinos then interact with other particles.

The same applies conversely to right-handed anti neutrinos. Because the neutrino spends only a short amount of time right-handed, while in flight, e.g., in the multi-folds where it can't interact, it explains why their mass is always small: they do not have the same opportunities as other to interact with the Higgs boson to acquire much mass through these interactions.

Gravity may suffice to address both problems with the Standard Model without all the other explanations that have been proposed beyond the Standard Model like Majorana neutrinos, and/or sterile neutrinos [400]. Section 9.11 will provide more considerations about mechanisms that would violate conservation of the Lepton number $L$.

\subsection{Gravity explains why three and only three generations per fermion families}

Inspired by [406], we propose some quick arguments that show how $\mathrm{SM}_{\mathrm{G}}$, or gravity added to the Standard Model as proposed in this paper can explain why there are three and only three generations per family of fermions (i.e., leptons and quarks).

Accordingly, we look at each fermion family sector of the Standard Model Lagrangian, we find that equation (22), (along with EPR entanglement contributions, just to see their impact), amounts to a term in:

$$
\mathcal{L}_{\text {int }} \propto m_{\text {Fermion }}+V_{\text {eff }}(\text { Gravity })+V_{\text {eff }}(E P R)
$$

We provided also the EPR entanglement contribution for completeness. The analysis below also shows that entanglement between the Higgs boson and leptons is probably not a significant enough contribution to matter. We rewrite it as:

$$
\mathcal{L}_{\text {int }} \propto m_{\text {Fermion }}+V_{1}+V_{2}
$$

or

$$
\mathcal{L}_{\text {int }} \propto m_{\text {Fermion }}\left(1+M_{\text {Higgs }} K_{1}+K_{2}\right)
$$

Where $M_{\text {Higgs }}$ designates the Higgs Mass. $K_{1}$ and $K_{2}$ depend on the interaction including the mass of fermion.

For $m_{\text {Fermion }} \approx M_{\text {Higgs }}$, the potentials are strong (strong gravity interactions).

For $m_{\text {Fermion }} \approx 0$, the effect is essentially null. In between the effect is essentially constant. This is illustrated in Figure 13 .

As a result, we see that three and only three generations of fermion masses per family make sense; it is what is observed. We managed to complete the intuition of the program initiated by S.Weinberg in [406].

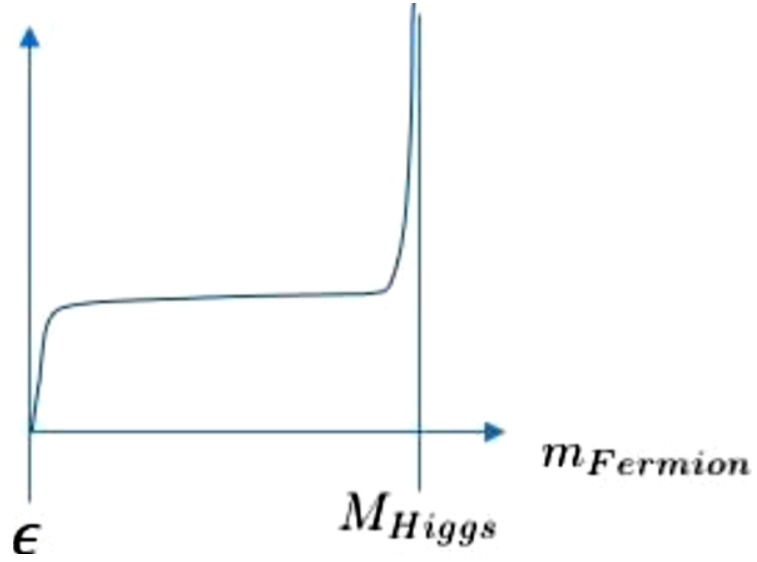

Figure 13: Plot of the value of equation (28) (vertical axis) that shows the three regions of Fermion mass in a family corresponding to three and only three generations per family: one with minimum mass, one with a mass close to the mass of the Higgs boson and one somewhere in between. It relies on estimating the impact on $K_{1}$ and $K_{2}$ and, in particular, the expectation that their value are larger when the mass matches the mass of the Higgs Boson. Only these three regimes are differentiated; any other kind of fermion in a family would have the same mass as one of these three and be hard to distinguish.

\subsection{No magnetic monopoles}

In section 1, we already mentioned that gravity renders existence of magnetic monopoles quite improbable as the Electromagnetic duality is broken by curved space $[342]^{142}$. Our approach reinforces that conclusion, with us establishing the viability and suitability of a semi classical analysis, valid at the proposed scales per the upcoming sections, and at even lower scales (magnetic monopole scales). This is the main dependency on $U_{M F}$; otherwise, it is an issue whenever gravity is present with, or without, our model. [342] risks invalidating in one shot all the models that predict monopoles [341], unless for those can still survive symmetry breaking by gravity. The Standard model is fine and not affected as it does not predict any magnetic monopoles. But any other model that describes a solution that must encompass gravity (à la superstring, quantum gravity or Theory of Everything), or that must coexists with gravity (i.e., occurring past expected decoupling of gravity from the rest; whatever the rest is) that predicts magnetic monopoles is probably doomed. Besides critically endangering the viability and confirmation of any theory predicting them, this conclusion also emphasizes the futility of hoping that the problem is only that we would be just waiting to discover them, or hoping having maybe (probably not) observed at least one magnetic monopole [321]. Magnetic monopoles probably just do not exist; that it be because the theories are not applicable to our universe, or because gravity ensures so. The reasoning above would still allow GUTs not including gravity even if they predict unobserved magnetic monopoles (if they can defend that they are still relevant despite gravity breaking the symmetry behind the motivations for magnetic monopoles); but then we also know that proton decay is 
another problem $[\underline{343}, \underline{344}]$. All GUTs predict magnetic monopoles one way or another to our knowledge [321]. Proton decay on the other hand may be avoided by some [482]. Also, as a result, charge quantization, if to be predicted, needs another justification. But, for example, it could be shown to result from the approximate symmetry that still exists in flat space [341], and this way, we can still explain integer and (fractional $(1 / 3)$ when confined) electric charges. Indeed, charges should not be affected by the presence or absence of gravity. We will repeat one more time that the implications are significant: no physics beyond the Standard Model should predict magnetic monopoles; unless if it can explain why such prediction is consistent with gravity or live with gravity destroying the symmetry.

Of course, in the latter case, it is possible to explain that a theory would predict monopole when ignoring gravity and that these would not more be expected in the presence of gravity. However, doing so would require that the rest of the model remain consistent (e.g. if magnetic monopole is the result of a symmetry that justifies fundamentally the model and that symmetry is broken by gravity, it is worth wondering if it ever got the opportunity to reign (as it could have been broken before ever coming into being) ...). Many theories beyond the Standard Model have quite a hurdle to overcome.

\section{STRUCTURES OF SPACETIME AND SPACETIME RECONSTRUCTION}

\subsection{Multi-fold universe as spacetime structure}

In spacetime for $U_{M F}$ is defined by $\mathcal{R}_{B G}$ : a pseudoRiemannian manifold equipped with the multi-fold mechanisms. For now, we have not tried to explain further how it is built and what are its own dynamics other than the multi-folds activation, attachment to entangled particles and deactivation mechanisms and the associated mappings ${ }^{143}$.

However, [189] argues convincingly that all the thermodynamics results for black holes and horizons (and the fact that they have hot temperatures) give strong indications that spacetime has a (discrete) structure. At the current scale of our analysis, in our model, the structure of spacetime is essentially only in the form of all the activated bundles $B_{\text {activ }}\left(x^{\mu}\right)$ of folds. A way forward may be to model this aspect, in terms of microscopic states, thermodynamics and (horizon) temperatures. We admit that this approach of keeping metric(s) and introducing structures of it (multifolds) is quite a step from the views and formalism so far in terms of fundamental microstructure to spacetime ("quanta of spacetime"). We already hinted that we believe that the description of spacetime by metric (and curvature) is approximate, or effective, at larger scale (quantum and macroscopic or semi classical and classical $)^{144}$. To progress, we are interested in reconstructing spacetime as if it did not exist before. By this we mean: spacetime does not exist yet or no particle has visited a region of interest yet, so that it has not yet been concretized (it does not exist in either cases) nor observed or occupied by matter, fields or energy ${ }^{145}$.
Past works like $[\underline{143}, \underline{190}]$ has shown that quantum information ${ }^{146}$ evolution seems to influence the behavior or nature of spacetime: entanglement between regions seems to create spacetime dips that might imply curvature (and hence gravity) or black holes and changes in entanglement modify the geometry of space-time. While not capturing anything close to the concepts of multi-folds, they hint why folds activation (by entanglement events) are actually plausible behaviors $^{147}$ :

- Entanglement dips (like starting a fold or black hole) locally the spacetime [190] in their graph model and so it is creating curvature

- Entanglement tends to bring spacetime region together [98], which can be seen as i) why gravity like attractions occur or ii) why the dips that initiate fold activation would try to form folds. Of course, limited to $\mathcal{R}_{B G}$, kinematics and dynamics of the next steps can't appear in these models.

These approaches therefore hint at relationships between black holes and folds deformations as well as the form and dynamics of the folds. Our approach is not just a thought process or an abstract intellectual exercise to find a putative solution to a (few) paradox(es). Instead, it is actually contained, at least hinted, in existing theories that have been validated or widely investigated (e.g. superstrings for the latter). In addition, these models are discrete graphs or networks, motivated by the desire to describe spacetime emergence; prior to the apparition of any metrics and when metrics should rather be treated as operators [484]. For all their simplicity and assumptions, these approaches really align well with what we have observed so far within $U_{M F}$.

\subsection{A first spacetime reconstruction model: a graph of microscopic black holes}

Inspired by these considerations, let us try to reconstruct the spacetime $\mathcal{R}_{B G}$, in $U_{M F}$, as a graph or network of microscopic black holes located at each node. We would hope that doing so actually reconstruct a spacetime, that can actually be or appear continuous, e.g., at larger scale where we do not see the stitching points where the spacetime surrounding each black hole merge, and that does not assume an initial metric or spacetime. Of course, each black hole will be associated to folds when activated by particles or energy, that excite it, as well as when entangled. The black holes are associated to a discrete scale of areas (of its horizon) for discrete levels of energy: treating black holes as a quantum object (Inspired from [382, 386] and the volume/area quanta in LQG based on invariance of Area and representations of Lorentz rotation group [25]). It turns out that such a model already exists and has been proposed in $[\underline{191}, \underline{192}, \underline{193}, \underline{194]}$ with graphs of Planck size microscopic Schwarzschild black holes, of elementary surface (Planck surface) multiplied by the eigen value expressed as eigenvalue of irreducible representation of space rotation (i.e., in $\left.\left(n+\frac{1}{2}\right)\right)^{148}$.

Assuming low energy (e.g. classical conditions for GR), [192] recovers the spacetime area law as well as Einstein's 
GR field equations. [191] illustrates the same in terms of geometry to recover weak field equations. Interestingly, at high energy, and so very small scales, the entropy recovered in a horizon of surface $\mathrm{A}$ is in $\ln \mathrm{A}$. This is in our view not a surprise as it means that, at Planck scales, entropy now tracks black holes as elementary quantum entities ( $\mathrm{A}$ is at that stage sum of the areas contributed by the black holes encompassed in it). It is important: when spacetime becomes discrete and scale is of the same order, the area law is replaced by particle state counting. This is also predicted, with a different formalism, in [195], and in section 7.4. This first-generation reconstruction of $U_{M F}$ is a valid, but coarse approximation at scales closer to Planck scale up to classical physics. It does not yet take advantage of all what we have learned so far ${ }^{149}$. Yet it illustrates already very well why in general semi classical models of gravity and spacetime are actually suitable down to scales way smaller than often expected. It also illustrates how a discrete spacetime with (quasi) black holes at every graph node seems to account pretty well for spacetime, with or without matter/energy in it.

\subsection{Selected prior works relevant to spacetime reconstruction}

To further our journey in reconstructing spacetime, let us enumerate and review a few additional prior works that will inspire, or motivate, aspects of our next steps. The list does not claim to be an exhaustive overview of the domain or of prior works relevant to this paper. Our plan is to borrow, in an upcoming section, from principles, or results, discussed below. They will help refine our reconstruction of the model of spacetime, discussed above when we will put it all together.

- Quantum spacetime emergence from the AdS/CFT correspondence conjecture, and entanglement [143, 190]. We already discussed these above. These works show that our approach is, in fact, somehow already contained, at least as hints, in Physics.

- Models where spacetime results from relationship and similarities in configuration space allow to re-derive Schrödinger's equation [199, 197, 198].

- Numerical methods and spacetime reconstruction models are discussed in $[243,407, \underline{408}, \underline{409]}$. They can be used to resolve $\mathbf{G R}$ on a lattice, or to reconstruct spacetime. Numerical methods for GR, including Regge calculus, are compiled in [410]. Tensor(s) (networks), spin networks e.g., [200, 201, 202], loop (quantum) gravity [30], spin foam [205] reconstruct spacetime from fluctuations of quantum areas and volumes guided by reconstructed Hamiltonians and Lagrangian. A wide comparison of these approaches is extensively discussed in [243].

- $[407, \underline{408}, \underline{409}]$ showed that spacetime reconstruction on a discrete spacetime converges only for a 4-D spacetime; thereby somehow explaining the dimensions of our universe. More arguments can be found as comments at [513].
- The simultaneous emergence of Schrödinger equation and essentially flat spacetime has been shown on a discrete (i.e., quantized) lattice with a minimum length (See for example [203, 204, 206]).

- Spacetime (metric, space and time points) emerging from (spontaneous) wave function collapse (when and where collapse takes place, spacetime appears) [208, 209]. Inspired by Penrose, we know that this could also be due to gravity, based on the hypothesis that gravity cannot handle superpositions e.g. [207]. In our model, we do not think that gravity is a systematic cause of collapse, as we explained earlier that curvature superposition does not have to be an issue in $U_{M F}$ ).

- Quantum fluctuations can also be the source of spacetime. [206] discusses how fluctuations in space amounts to generate particles that follow wave functions equations (i.e., Schrödinger equation), and how fluctuations in time creates a spatial perturbation that can be modelled as a Klein Gordon field (e.g. Bosons with no spin are modelled in that paper, or Fermions) allowing now for multiple particles to appear. Furthermore, around the place where the first particle is located (at a distance $\varepsilon / 2$ in that paper terminology, that one can interpret as $\left(\varepsilon / 2 \approx l_{p} / 2\right)$, where $l_{p}$ is Planck length), a Schwarzschild spacetime appears with a metric and curvature. We will note that we are back to the microscopic quasi (Planck) black hole surrounding each particle hinting consistently at our multi-fold universe $U_{M F}$. And again, it looks like these aspects of our approach are contained, at least as hints, in existing theories of Physics. The presence of the Klein Gordon field can account for multiple particles, and it denotes that if the first particle resulted from the fluctuation, then its entangled anti particle did also. And as discussed in [24], particles can be followed in QFT and modelled as long as one studies the action of the field operator on a multi particle wave function associated to the different particles; variable amounts of particles solely reflect that fact that particles can appear or disappear aside from the ones that we follow (and that are still not yet dead). We already know that this works well in $U_{M F}$.

- In terms of dimensions of spacetime, [232] has argued that, at very small scale, spacetime and gravity processes seem to reduce in dimensions (i.e., in terms of degrees of freedom). [232] stops at 3-D, but, as above the energy of the electroweak scales, everything is massless, then the effect evolve to become 2-D and higher energies.

All these results help and guide in how to better reconstruct $U_{M F}$ that what we did earlier.

\subsection{Random walks, (multi-) fractal and fractional spacetimes and Path Integrals}

We have seen how central the concept of Path Integral to Physics is in general, as well as to characterize $U_{M F}$. Yet, early on, Richard Feynman [46] observed that the trajectories of particles, in particular relativistic ones, look like 
Brownian motion ${ }^{150}$. We know that random walk fluctuations are similar to Brownian motion. This observation led to the alternate ways to derive for example Schrödinger equation presented in for example [212].

Ordt [211] then showed that one can model such paths with a fractal spacetime. Ordt work, and follow-ups, recover a relationship à la de Broglie, the uncertainty principle, Lorentz covariance, and the Klein Gordon and Dirac equations $[\underline{211}, \underline{212}]$. Separately, but of course directly related, it is worth noting, as others have, that particle physics and interactions as modeled by Feynman diagrams, also the outcome of Path Integrals, are fundamentally fractallooking [411]. The one (spatial) dimensional work in [211] seems to point to a possible alternate way to understand and account also for the different paths of the Path Integral:

If spacetime was to be fractal, then a particles could take fractal paths to go from a location to another and that could bring them all over (or significantly all over) the place: something reminiscent of the Path Integral (that could then possibly appear as an approximation of model of that behavior in an non fractal or continuous world ${ }^{151}$.

Of course, fractal concepts in the universe only fit a finite range of scales ${ }^{152}$. On the other hand, the giant structures recently observed in the universe, as detailed for example in [492], could be also footprints of random walk paths enlarged by inflation. The original observations of fractal, and multi fractal, structures have inspired work around scale relativity $[\underline{415}, \underline{416}]$, and fractal spacetime proposals initially motivated by [211], as well as the implications and numerology approaches that can be obtained by simply postulating a fractal spacetime [417, 418]. Entry points to these works can be found at $[\underline{419}, \underline{418}, \underline{421}, \underline{420}]$ including, in particular, discussions and references of the Cantor-like $E$-infinity fractal spacetime. In a fractal or multi fractal spacetime, it is possible to develop calculus and physics: fractional spaces can be regarded as fractals when the ratio of their Hausdorff and spectral dimension is greater than one $[\underline{425}, \underline{426}, \underline{438}, \underline{429}, \underline{427}, \underline{428}]$. Relevant to our activities we note:

- Einstein's GR equation, and solutions including black holes have been formulated in fractional spacetime. See for example $[\underline{422}, \underline{423}, \underline{424}]$.

- Quantum mechanics has also been defined over fractional spacetime with Schrödinger, Dirac and other field equations, and Fractional space Path Integrals. See for example $[\underline{430}, \underline{431}, \underline{434}, \underline{433}, \underline{435}, \underline{436}, \underline{437]}$. We note in particular how effective potentials [432, 433], albeit usually complex/imaginary (maybe a hint that the Higgs boson, with its complex field, and imaginary mass, would be the, or a, main contributor to these random walks above the electroweak symmetry breaking, where 2-D then 3-D dominate, before 4-D), also play a role in emulating the impact of fractional dimensions on the Schrödinger equation. The consistency of the Path Integral in fractional spacetime obtained in $[434,436]$ is a key result that allows us to extend our models to such spacetime.

\subsection{A case for a discrete spacetime in $U_{M F}$}

So far, $U_{M F}$ has been handled as a continuous space; except for a few comments about small scale cut offs (usually to avoid singularities or divergences), and our repeated mention that we may want to, and will, quantize the approach to find gravitons and to support the existence of entropy for horizons or spacetime. We already mentioned that usually the challenges met by quantum gravity approaches primarily come from the ultraviolet divergences and the difficulties to renormalize the models [44]. An artificial, or phenomenological, way to address the problems with gravity, or QFT in general, is to introduce an UV cutoff that often is assumed to be related to Planck length $l_{P}$. This is typically motivated by some assumptions of discretization of spacetime, or at least of minimum length.

Different methods have also been introduced to reconstruct spacetime that it be as a discrete, or lattice model, or lattice model or for numerical analysis of GR [년 $\underline{407}, \underline{408}, \underline{409}$, 410]. Works like LQG with spin networks, and spin foams, and variations $[\underline{200}, \underline{201}, \underline{202}, \underline{30}, \underline{205}, \underline{25}]$ try to fully reconstruct spacetime from discrete networks built or evolving according to Hamiltonians and Lagrangian constructed on quantum geometric or Action hypotheses.

It has also been shown that non-commutative geometry [441] and non-associative geometry [440] creates geometry without (or with a fuzzy) spacetime ${ }^{153}$; which may be what happens at Planck scale. Indeed, different works allow to build decent frameworks. With non-commutativity and nonassociativity, chances are significant that spacetime is discrete. That being said, some strong objections exist against a discrete spacetime. It is based on the view that background independence (i.e., related to general covariance) and Lorentz invariance will often be lost in such a space. This is not necessarily true as discussed in $[\underline{442}, \underline{443}$, $\underline{487}, \underline{488}, \underline{486}, \underline{444}, \underline{445}]$, where it is shown that noncommutative geometry can lead to preservation of Lorentz symmetries as well as discrete spacetime ${ }^{154}$. In a constructive manner, this can be done by defining explicitly a covariant Lorentz tensor as the commutator, inspired by [442]. [487, 488, 486, 489], constructed some explicit models.

In particular, they provide proof that we can have a Physics well defined, including Path Integrals which are well defined, and again, respect the required commutator relationships. If Lorentz covariance is satisfied and space time is discrete, we know that the maximum speed ensured by discreteness (or minimal length in spacetime) is also Lorentz invariant.

However, at very low scale or when spacetime does not exist yet (or has not yet been observed), and if it was discrete, it is unclear if Lorentz symmetry or background independence are criteria to apply.

For example, any spacetime poorly populated in nodes or limited in size would not be Lorentz invariant/symmetric, by definition. Yet, for a discrete spacetime, [446] argues that the only distribution of points in spacetime that is Lorentz invariant is a random (Poisson) distribution. Randomness is the way to ensure Lorentz invariance when all other 
mechanisms fall apart (i.e., especially in the earliest moment when not many points have been concretized, and random walks are all what matters). Lorentz invariance, with $c$ invariance and Special Relativity really result from this discrete structure and random walks, which support Path Integrals. This is quite a result, from the ground up!

Another concern often cited is the loss of the notion of the euclidian-2 norm in discrete space (i.e., the Pythagorean theorem), as presented by Weyl, with the Weyl's tile argument [447]. Weyl argued that discrete spacetime would not satisfy the Pythagorean theorem and, because such theorem, and, because such theorem has been always, so far, validated to any degree of accuracy, spacetime cannot be discrete (or we would have run into problems already).

This paradox has since been addressed [448] (And there are other analyses addressing it also), thereby removing one of the toughest arguments against a discrete spacetime. The Poisson sprinkling can also be invoked.

Eventually, some have also raised the concern that in a discrete spacetime, Path Integrals would lose the commutator relationships mentioned earlier [247]. It is not the case with fractional spacetime, where correct fractional Path Integrals can be defined as well as uncertainty principles [435]. In a discrete environment, they can also maintain their Lorentz covariance [497].

What would lead us to believe, based on what we have discovered so far, that $U_{M F}$ spacetime could be discrete (in ways only detectable or relevant at very at small scales)?

Let us consider the following, based on the above, and what we have learned already about $U_{M F}$

- The absolute prohibition of supra luminous signal seems to indicate that spacetime is discrete with random jumps between discrete nodes. It's Lorentz invariance is also critical to obtain at least at Quantum scales, and above. There are not many mechanisms to ensure that. Yet, above, we described a relatively simple one that will guarantee such features.

- In our model for $U_{M F}$, the carrier of gravity seems to be attached to EPR entangled virtual particles sourced from a real particle or to EPR entangled real particles, as folds or bundles of activated folds. What we conventionally take for a graviton effect is the resulting effective potential $V_{\text {eff }}$ propagating in spacetime; there simply are no other option in a world not aware of multi-folds! These multi-folds mechanisms have not yet been quantized in this paper but have a $180^{\circ}$ symmetry which matches spin-2 symmetries announcing quantized gravitons. If we want to pursue this hunch, then we will need to quantize the folds (i.e., their sizes). It would mean discretized multi-folds. In order to make the mappings consistent, it would logically imply that $\mathcal{R}_{B G}$ is also discrete; and, conversely, a discrete spacetime implies a discrete multi-fold. It leads to our, often argued but so far not yet justified, argument of equivalence between quanta of spacetime and quanta of multi-folds living in $\operatorname{AdS}(5)$.
- The contribution to $V_{\text {eff }}$ (of a single fold) is only ${ }^{155}$ the Ricci curvature scalar. This can be argued as a signature of a discrete spacetime [25].

- Because the mapping and folds at a given time imply that a grand circle is equal or proportional to the distance travelled by the corresponding entangled virtual or real particle in $\mathcal{R}_{B G}$, it means that, for the folds, space coordinate and momentum are proportional to each other. In Quantum Physics, this means that spatial coordinates for the graviton do not commute; a signal that the space where graviton lives in, and the background spacetime $\mathcal{R}_{B G}$, per the previous points, are rather fuzzy and are probably discrete. In any case, the geometry of $U_{M F}$ is non-commutative.

- The Area laws for black holes and suitable spacetime horizons (e.g. causal), and inequalities otherwise imply a microscopic structure of spacetime, that has to be quantized, or discrete, to be explained (otherwise, what would be spacetime). In addition, [491] shows that at least for 1-D systems, the presence of a gapped lattice (i.e., a discrete spacetime) suffice to lead to Area laws for entanglements. While for higher dimensions, the systems may not satisfy area laws, as we saw, this principle is a strong guidance that spacetime is discrete, especially if essentially 2-D at small scales (see below).

- It is great that our approach derives non-commutativity. Yet was already known that it was a consequence of adding an uncertainty principle to GR. [487, 488] also detailed why gravity, as semi classical GR, and Quantum Mechanics, imply non-commutative geometry. Indeed, the more accurate is positioning, the bigger is the momentum uncertainty, and, therefore, the Einstein stress energy tensor, that results, generates a gravitational field described by Einstein's GR equations. When the gravitational field becomes so strong that it prevents light, or any other signals from leaving the localization region, an operational meaning can no longer be attached to the localization: black holes appear. Just as we already discussed for spacetime, and particles. Computing the resulting limitations, they found commutations rules expressed as a tensor whose components commute with all coordinates. So, the presence of gravity makes the spacetime effectively noncommutative, and this feature should be present in any quantum theory of gravitation. In $U_{M F}$, the same reasoning applies the same way.

- Entanglement mechanisms are, a priori, non-associative across entangled particles (e.g., think of the hierarchy discussion earlier on in section 4.2). Another sign (that we will not try to detail further here) of a probable discrete spacetime.

- Although in the approximations of our first-generation reconstruction shown in section 9.2, the resulting reconstructed spacetime could very well be continuous; yet what we proposed was by construction fundamentally discrete (and without initially any notion of metric), and only appearing continuous because the micro black hole 
offer a continuous spacetime at, and outside their horizon ${ }^{156}$.

- If we follow a random walk model, something that matches the path of relativistic particles driven by their Path Integral, then spacetime looks like a spacetime with fractional dimensions, with $D \approx 2$ [415]. As $U_{M F}$ is built on Path Integrals, a reconstruction approach based on random walk would make sense, and be fractal, hence discrete. We note immediately that in such a case, at small scales, where multi fractal behavior is visible, we would recover the dimension 2 predicted in [232]. Fractal spacetime also recovers the transition between 2 and 4 dimensions as well as the existence of a maximum speed converging towards $c$ as scale increases $[\underline{419}, \underline{418}, \underline{421}$, 420]. 4-D is also the first stable dimensions obtained by numerical reconstructions $[\underline{407}, \underline{408}, \underline{409,513}]$.

- As particles are surrounded by (quasi) ${ }^{157}$ microscopic black holes in $U_{M F}$, and / or, as they are themselves microscopic black holes, the above leads us to expect that the particles evolve in random walk, with random walk with random discrete steps. These steps could leave microscopic black hole footprints (instead of remnants with their connotations) everywhere: they "create spacetime" by leaving footprints ${ }^{158}$ as minimum sized, i.e., Planck scale black holes, holding the entropy to area laws, and/or area eigen function rules, as proposed in section 9.2. Alternatively, the black hole nodes could be the result of collapse of the wave function as proposed by $[208, \underline{209]}$.

- In section 9.3, we saw that uncertainty perturbations of spacetime can create particles, fields and / or black holes around them. With this, random walk can create new spacetime (as nodes in discrete mode; with surrounding microscopic black hole), or continuously, as well as new particles.

- At very small scales, if an area is a countable measure of the number of spacetime quanta in the system, it is normal that entropy would become proportional to $\ln \mathrm{A}$ as observed with our first-generation reconstruction. It is what happens when the dimension of spacetime behaves like 2-D: the nodes $=$ the degrees of freedom. $\mathrm{A}$ is the sum of the nodes' degrees of freedom. At larger scales gravity matching energy, or mass, appears as effective curvature or potential, with the multi-fold mechanism, and it is when the area laws appear.

So, reasoning from these considerations and the properties of $U_{M F}$, we conclude that spacetime is probably discrete, built by particles, that behave and/or are surrounded by microscopic black holes and propagate by random walk with hop speeds limited to an upper value (invariant per Lorentz invariance resulting from the randomness). Doing so, their presence and passage creates spacetime in the form of Planck size black holes. These black holes are discretely positioned in a multifractal pattern. The random distribution of the hops ensures Lorentz invariance and behaves essentially as a 2-D process.
At such scales, spacetime is fuzzy (non-commutative and non-associative) because of uncertainties, which also ensures Lorentz invariances/symmetries, as we look from larger scales. At larger scale, they appear continuous, hence semiclassical models can work till very small scales. By the way, such a spacetime discreteness was obvious all along! Indeed

- The principle of absolutely no supra luminous moves or interaction amounts to a hard filtering of momentums for all processes. This means a discrete spacetime per Shannon's theorem [449]. This is actually the reasoning based on Shannon, forget the reference picked to motivate the statements, that was behind our initial belief that the no supra luminous principle is key to resolve the issues of gravity related singularities or divergences; even before most of the rest of our analyses were done. It does not hurt that once spacetime is discrete, one can easily understand why there is a maximum speed for everything.

- [450], could also have told it all along with its analysis of black holes, and ultimately the same argument of cutoff coming from another analysis of Shannon's theorem.

- Gravitons would not exist as quanta of spacetime, if it were not discrete, that they be particles, as seems to rather be the case, or even unphysical.

- As we already said, explaining entropy would be a problem if not associated to a microscopic discrete structure $^{159}$.

\subsection{Second $U_{M F}$ spacetime reconstruction from random walk, a fractional dimension spacetime at Planck Scale, and black holes as spacetime points and particles}

Once again, let us put together all the lessons learned before in order to fully describe $U_{M F}$ at all scales. For this, we will try to reconstruct spacetime as a fundamentally discrete with fractional dimensions due to it originating from random walks, and with multi-folds. Nodes of the spacetimes are microscopic $\sim$ Planck Schwarzschild black holes, as in our first reconstruction attempt and particles as themselves black holes, consistent with our analysis so far. For the sake of discussion, let us start with $U_{M F}$ before it even exists: there is no spacetime $\mathcal{R}_{B G}$ and no particles or energy other than at one point (of a certain thickness because of uncertainty-so there are probably a few points separated by $l_{P}$ ). Alternatively, it can be at a set of points, if that was the initial condition of our multi-fold universe. Minimum length and uncertainty, torsion, or as we know that several models like LQG predict absence of singularity, and big bounce from another "universe", make the latter also quite plausible ${ }^{160}$. For now, let us not worry about the cosmological aspects, and therefore not discuss the energy levels required to get this started and what happens at what regime.

We will assume that, through uncertainty, a time, and/or a space perturbation takes place. The time perturbation creates 
a first-or new-time interval (a second point (or a new point)) and the space perturbation creates a particle or particles ${ }^{161}$ with wave functions that spans areas larger than the microscopic black holes of the first-generation model. The space perturbation implies one, or a few new spatial points associated to the new time. Their positions in spacetime are uncertain within the domain of the wavefunction of the particle, so it may be one or another point that is occupied. The process can repeat, at each point. All these particles are massless (think of first moment of the big bang - it does not matter that we argue before or during inflation if inflation exists $[167, \underline{165}, \underline{452}, \underline{453}, \underline{454]})$ : it means that the particles all moves at the speed of light between past points and new points. If some particles are massive fluctuations, they will move slower; but probably none are massive at the beginning, if we understand the cosmology timelines well enough $[167, \underline{165}]$. Being relativistic, the movements of the particles, now at, and from, different points of this initial graph of spacetime, appear as random walks in space and time. That means 2-D (1-D in space $+1-\mathrm{D}$ in time) paths (with respect to say a cartesian reference frame, now possible as hops concretize the notion of distances and minimum distances) at the speed of light (or at slower speed which just amounts to not space hopping with every time jumps). Another way to see that the process is 2-D is to remember that when moving at $c$, particles are flattened: they live in 2D orthogonal to their displacement and, what matters for gravity and creation of spacetime, they move randomly in 1$\mathrm{D}$, with the 1-D clicks in time (time randomness is key and the essence of the insight of Ordt [211], when he proposed to give it a fractal model.

We recover the 2-D prediction. Also, note that $c$ could be larger than, or different from $c$ : the path is fractal ${ }^{162}$, all what will matter is what the conventional resulting speed (at quantum or classical scales) is $c[\underline{419}, \underline{418}, \underline{421}, \underline{420}]$. The paths are described by (Schrödinger,) Klein Gordon or Dirac equations, and the resulting spacetime rapidly becomes Lorentz invariance through the random sprinklings of points and non-commutative fuzziness that prevents knowing which points exactly exist among its neighbors within some uncertainty range. After a while, we can assume that a fractional (multi fractal within a certain range of scales) spacetime has been built by the passage of the particles [ $\underline{419}$, $\underline{418}, \underline{421}, \underline{420}$ ], and Physics can be well defined in such spacetime, including Path Integrals, that motivate the existence of the multi-fold mechanisms also at such scales: gravity, and EPR entanglement can take place. Commutators rules are maintained between position and momentum (operators) making the Path Integral well defined. Noncommutativity and randomness ensure Lorentz invariance. As we raise a bit in scale, spacetime appears to rather be 3-D then 4-D, and with $c$ as speed of light, and absolutely no possibility for anything to propagate faster when looked from such a scale upwards. It is a consequence of fractal spacetime [419, $\underline{418}, \underline{421}, \underline{420}]$ where 4-D is predicted by numerical stability of $\mathbf{G R}$ solutions built from scratch $[\underline{407}, \underline{408}, \underline{409}]$ (as we did, in our case by random walk, not as GR solution, but with the multi-fold mechanism that we know to lead to $\mathbf{G R}$, a proof has since been provided later at [517]). As soon as at least two or three different spacetime points exist, the fold mechanisms can appear and enable entanglement between the particles (real and virtual). It is sketched in Figure 14.

a) $\Delta t$

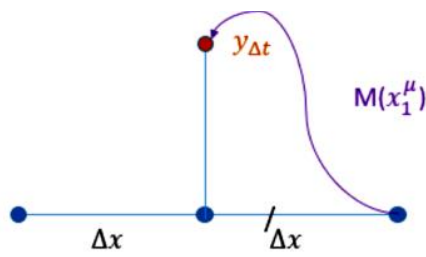

2) $2 \Delta t$

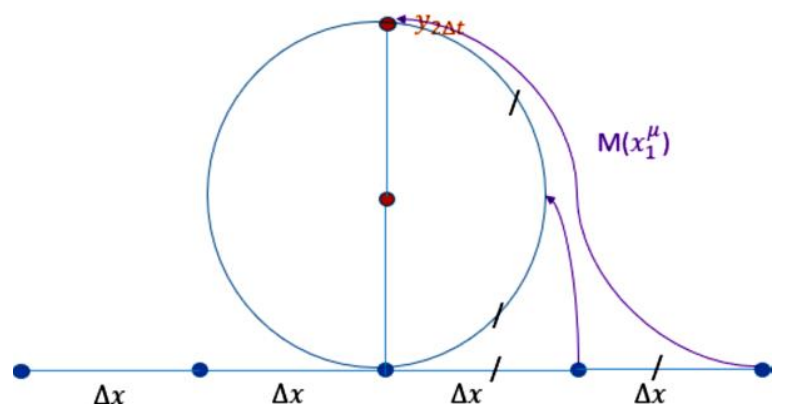

Figure 14: Growth of entangled spacetime by random walk or at inflation has spacetime points entangled locally at least for a while. The growth in spacetime matches the growth of the multi-folds. These deltas in spacetime and in folds are the quanta of the multifold mechanism (growth of the multi-folds), on in each fold, per (random) time click. They match the space-time growth or changes in $\mathcal{R}_{B G}$ encountered in conventional quantization of gravity/GR) and of gravity as a result. These are the gravitons in $U_{M F}$ : in our model, they live outside the spacetime $\mathcal{R}_{B G}$ but match the perturbation and growth in $\mathcal{R}_{B G}$.

As the graph grows, spacetime quanta are added/created, and neighbors are entangled. And so, multi-folds build between the points as well as between points and particles that left them. They are discrete, and grow by the same quanta: gravitons are quanta of "node segments added to spacetime and to multi-folds": a new point is matched with a new point for the folds and a new time click grows the corresponding multi-folds. Gravitons are the minimum of such addition per time click: a quantum of addition (or perturbation) of spacetime in $\mathcal{R}_{B G}$, matches a quantum of addition of spacetime in the multi-folds.

This equivalence (one in spacetime, one outside not necessarily involved in energy conservation) means that the two points of view can be taken: these quanta or gravitons are spacetime minimum perturbations, or multi-folds quanta. Our analysis, where we argued that the latter works, and explains how and why it matches with, and how it relates, to the conventional quantization of GR via linear perturbations and models by say Gupta, Feynman and Weinberg mentioned earlier: the point of views is equivalent. Yet our proposals avoid the divergences and renormalization problems. Gravitons and all particles live in an 
anticommutative geometry because of its non-zero commutators.

As already discussed, with random walks, and random distribution as well as noncommutativity, Lorentz invariance appears, and $c$ is the maximum speed for anything. As gravitons are also the quanta of spacetime, the initial steps of the spacetime emergence, by random walk, create such a fuzzy noncommutative geometry, random and multi-fractal, with Lorentz symmetries baked in. It is the dual view of the Path Integral formalism (for the right Action), and all its implications in physics.

Unless imposed by different initial conditions (e.g. from a big bounce), the spacetime $\mathcal{R}_{B G}$ starts flat ${ }^{163}$. Impressions of curvature (see our earlier discussions) appears at a higher scale. This allows us to recover notions of Quantum mechanics, and Classical Physics with Path Integrals and wave function equations in flat spacetime and notions of curved spacetime for semi classical or classical GR.

Because we do have a discrete spacetime, no gravity/singularity appears. Yet, effective potentials due to fold curvature appear and black holes without singularity can exist. At larger scale, when we lose the details and models with theories like GR, (artificial) singularities may appear, to be handled by cutoff. They are the result of the approximations made. It is just like conventional QFT with its lost background independence, or not filtering out space like path in its Path Integrals. It just does not matter, as long as we understand the impact of these approximations and why we can ignore them in calculations and when we should not in some models or theorems.

In addition to the discreteness considerations, each node has a local microscopic Schwarzschild black hole as discussed in section 9.2. Each particle is also modeled as a microscopic black hole with a mass, rotation (spin) and charges [ $\underline{387}, \underline{384}$, $\underline{385}, \underline{383}$ ]. We know that such a model creates some horizon dimension and extremality issues (with possible naked singularity in conventional physics) [387]. However, this is forgetting how.

- spacetime discreteness, expansion (see later) and torsion can get us out of trouble (no naked singularity in our world for even for extremal black holes and beyond).

- more important in the scope of the present formalism, the fact that at very small scales, massive gravity may significantly increase the effect, and therefore reduce the horizon estimates so that they fit better known particle size estimates and further resolve bad horizons. This recovers horizon sizes closer to observation (e.g., for the electron) with quantum models. It also puts all these behaviors within the uncertainty region of the particle and so it does not really matter: each model is acceptable, and we may even switch models on the flight based on what we want to discuss without negative consequences!

The idea is that particles are black holes described by their usual equations, with spin as an internal term coming from the wave function rotation generated or captured by the entry of the surrounding virtual particles in the multi-fold. As they move, they imprint spacetime leaving behind an elementary
Planck black hole as in section 9.2. The position is one, or many, of the cartesian points where the particle appeared to be, give or take the uncertainties. This model recovers Einstein's GR field equations, Area and $\ln A$ laws for entropy of horizons/surfaces [191, $\underline{192}, \underline{193}, \underline{194]}$. Yet, at very small scales, the nodes of the model are now dictated by the fractal distribution from the random walk with uncertainties, which salvages Lorentz invariance till almost the first moments or till at Planck length.

\subsection{Discretized spacetime matters}

In section 8 , we showed how semi classical consequences of gravity in $U_{M F}$ added to the Standard Model could start to help clarify some of its current open problems. The same is true when bringing in the discrete nature of $U_{M F}$ at very small scales.

We know that it matters, because it resolves the problems of gravitational singularities: they do not exist anymore, that torsion and spin coupling to gravity be involved or not.

Black holes still have their strange properties, especially when seen in a macroscopic model like GR, but singularities are never present in reality. We do not know how the universe started (e.g. from an singularity or not) but it certainly cannot return to one; it would rather bounce into a big bounce. Closer to our subjects of interest, with the microscopic black holes model on discrete spacetime node and black hole particles (and no singularity concerns), we now have a way stronger basis to apply semi classical model even at particle scales. Leading to for example the considerations of section 8 . It also shows that numerical and lattice models can actually be the correct models (i.e., not just ap- proximations biased by effects that would carry systematically wrong features, as Gibbs oscillations due to the discrete approximation of the lattices, and that never disappear or reduce their effect until the limit to continuous is taken. These approximations can plague many GR numerical solutions, or simulations in LQG spacetimes reconstructions for example [25, $\underline{83}]$. Yet, as spacetime is discrete in $U_{M F}$, these effects may indeed physically exist even if probably also tamed by the uncertainties, random walks instead of regular patterns, and with a spacetime provided by the microscopic black holes. As consequence, our view of the work of causal dynamic triangulation and reconstructions of spacetime validly demonstrates why our spacetime is 4-D: the are artefact of lattices that caused this observation are correct $[\underline{407}, \underline{408}, \underline{409}]^{164}$. Of course, per our advices to all quantum gravity theories, Causal Dynamic Triangulation [459] would benefit to add better notions of entanglement, multi-folds (can be emulated by Action), and random walks with fractal seed structure. . .

As a next example, let us go back to the Standard Model and Yang-Mills theory as a non-abelian quantum field theory for QCD. It is well known that the mass gap in Yang-Mills theory [460] is an open problem that has not been resolved: proving that the lowest energy state of energy is above the vacuum (with a mass gap) proves the stability of the theory and possible existence of Yang-mills theory, as well as for example the viability of glueballs. None of these have been 
validated so far. Only lattice simulation-based proofs have been achieved [461]. In $U_{M F}$, we believe that with our approach the hypothesis has been proven. Indeed, the result in [461] is now sufficient as it shows independence of the lattice cell size. To be sure, repeating the simulation down to maybe $\sim 10^{-18} \mathrm{~m}$ would settle it for sure as $\sim 10^{-16} \mathrm{~m}$. Progress in computing systems should make it possible. But we know that spacetime in $U_{M F}$ is discrete. So, it is certain that in $U_{M F}$, such results can reflect reality and no systematic discrete biases. In fact, solving the mass gap in continuous spacetime may not be possible nor lead to the right result: in continuous spacetime there may or may not be a mass gap, who cares? For proof and discussions see papers at [513].

\subsection{What about a weak gravity conjecture in $U_{M F}$ ?}

A consequence of the model of particles as microscopic black holes from section 9.6, with massive gravity, is that as massive gravity plays a larger role, it amounts to having Newton's gravity constant $\mathbf{G}$ increase. This could reduce extremal conditions in the Reissner-Nordström metric [381]. Yet the charge of particles (e.g. electron) is often larger than what is allowed for its mass in order not to be extremal in conventional GR (i.e., involving only massless gravity contributions)[387]. This observation has some consequences and in later papers tracked at [513], we will see that (over) extremality is not an issue:

- It fits one of the consequences of the weak gravitation conjecture proposed in [475]. The weak gravitation conjecture was saying that for gravity or quantum gravity to be consistent, the force of the field gravity must be weaker than that of any of the gauge field forces, in suitable units. They argued that there always must be "elementary particles" for which the ratio $(\mathrm{m} /|q|)$ of their mass over their gauge field charge (e.g. electric charge, magnetic charge) is smaller than one:

$$
\frac{m}{|q|}<1
$$

- (In natural units). The latter is indeed the case with electrons, in conventional physics.

- Yet, it is unclear if the validity for a given particle (e.g. electron) implies that gravity is always strictly weaker than any other Gauge interaction (e.g. electromagnetism). Indeed, we just argued that it works because $G$ increases significantly at very small scale because of massive gravity. It is behind the semi-classical implications with the Standard Model in section 8, and our explanations above. It seems rather that we argue that gravity and electromagnetism rather reach a similar (in fact exactly the same in natural units) value, so that particles can be stable microscopic black holes with no more contraction or expansion, other than for uncertainty reasons; but not evaporation, and the microscopic black hole is extremal and stable.

- We also know that conventionally, evaporation of macroscopic blackholes, if charged, will probably render them extremal at some point, despite $\sim$ Schwinger effects, and then they can no more evaporate, and we would have problematic remnants [476].

- If extremal blackhole can't evaporate, then they can still break into smaller black holes. [477] argued that to do so, they would split into a non-extremal black hole (that can continue to decay by evaporation) and another blackhole who would be beyond extremal; something that they argue would be possible only if physics changes with smaller black holes so that quantum gravity effect allow more charge per mass for them. But in $U_{M F}$, we would be ok with that. (Again see papers at [513]).

So, we do not believe that it is what happens in $U_{M F}$. Indeed, a non-extremal black hole will decay as in conventional physics. When it becomes extremal, instead of decaying by evaporation, it can break apart into smaller and smaller extremal black holes (e.g. they split in two then again and again) randomly. At the end, they will end up into elementary particles: no remnants; no mysteries, no paradoxes; no problems. So, in $U_{M F}$, at very small scales, i.e., at particles horizon scales and smaller, gravity becomes of the same order, in fact possibly the same coupling value, as the other interactions, due to the short-range massive gravity effects (and entanglement, although that does not seem to contribute to the gravity strength beyond the virtual particle entanglement effects around a source in our approach). We do not strictly satisfy the weak gravity conjecture despite having particles satisfying the mass to charge ratio expectation.

On the other hand, the spirit of the weak gravity conjecture may remain valid in $U_{M F}$, in the sense that, as discussed in section 4.1, the probability of a particle's paths venturing in a fold is very small as to preserve conservation laws and unitarity. Also gravitons live in $\mathbf{A d S ( 5 )}$ rather than our spacetime. The outcome is a weak impact at most scale above particle uncertainty dimensions (and the horizons of particle as black hole). Around these scales (after gravity get a strong massive contribution), it evolves differently. Of course, that is different formulation from [475]; it is no more the same conjecture as gravity does not have to strictly be the weakest force at all scales. This again means, in our view, an impact on how superstring theory should look at their landscape and swamp [61].

\subsection{A New Black Hole Life Cycle Option: From Quantum Evaporation to Extremal Disintegration into Extremal Black Holes, Down to Microscopic Black Hole Particles}

Inspired by the conclusions in $[\underline{477}, \underline{478}]$, it should be clear that while the massive gravity and entanglement decreases entropy, splits of extremal black holes into two extremal black holes increases the total entropy without the need for hypotheses of corrections of physics for smaller black holes, i.e.,

$$
d E_{B H_{1}+B H_{2}}=0
$$

in the first law of black hole thermodynamics [479] implies: 


$$
d S_{\text {Total }}=0_{\text {for }(B H 1+B H 2)}+d S_{2-\text { systems }} \geq 0
$$

Therefore, it is a normal evolution.

We have an important new claim in $U_{M F}$ : black holes evaporate until they disintegrate into smaller and smaller extremal black holes down to elementary charged particles. This is also without problematic remnants issues [476]. Also, it should alleviate concerns about unitarity and information paradoxes.

\subsection{Interaction Democracy extended to Gravity or Ultimate Unification?}

The match in intensity of gravity with the other forces reminds us of the arguments used for GUTs, and GUT scales [236]. However, now it is between gravity and the other gauge interactions: Strong (or QCD) and Electroweak. At the scales considered, their charges are involved, with (no more-see papers at [513]) confinement as needed that maintains colors hidden, or rather neutralized away from the horizon). Because gravity is involved, there are no magnetic charges (aka magnetic monopoles). The consequences are that gravity, strong and electroweak interactions strength converge at the small scales we discussed: a sign of unification. In fact, to our knowledge, it may be the first time that such a reasoning or convergence involving gravity could be made, albeit in $U_{M F}$. So far, our thought process did not provide actual arguments of a symmetry breaking mechanism. Of course, there can be many candidates, including, for examples, effects of scales jumping for example from mostly 2-D to 4-D or from mostly discrete to continuous or from non-commutative to mostly commutative behaviors in $\mathcal{R}_{B G}$. The list is long, and future work will single out the massless Higgs complex field, with imaginary mass (See papers at [513]).

Yet all we can conclude, at this time, is that the intensity of the interactions (coupling) seems to converge:

- Between gravity (with massive (and entanglement) contributions), and Electroweak.

- As well as with the strong interaction. There are no signs (to the contrary because among other things of the challenges with proton decays and magnetic monopoles) that QCD and Electroweak interactions unify first, even if, conventionally, their intensity seems to converge (earlier) [236].

We can therefore formulate two different hypotheses:

(1) We only have a democratization effect IDeG: all interactions have roughly the same intensity at very small scales. They may remain distinct.

(2) They may instead be facets of a same mechanism; still unknown. The mechanism is an Ultimate Unification (UU) interaction. Symmetry breaking(s) lead(s) to these different facets.

The first hypothesis (number 1) is what we have deducted so far and seems to apply in $U_{M F}$. Can we find hints about (2)? If we follow our reasoning, IDeG results from massive gravity taking a larger role. In truth massless gravity probably also increases (as more massless carriers are involved, and distances are very small). In fact, beyond electroweak unification scales, everybody is kind of massless; so, the statement (at the reasoning about massive gravity at very small scale made so far-it was a sleigh of hand) is somehow ambiguous: at these scales, effects are short range but carriers are massless; just not dominated by virtual photons (and virtual neutrinos). All these carriers are involved on the same footing. Isn't that the unification we are looking for? Every particle equally contributes virtually to carrying entanglement, and therefore gravity like effects, while it can equally carry, or generate, or participate to electroweak, and strong interactions; all or some with charges.

Of course, in such a picture, all these interactions will have intensities of the same order of magnitude, in fact the same intensity, as being massless, they kind of all have similar probability to appear. That is the Ultimate Unification (UU), and the symmetry breaking occurs when scale increase and continues when masses appear. But the fundamentals of UU is democratization of the entanglement and gravity effects across all sources and carriers, all massless while they go on their own business; therefore, matching each effect particle by particle, carrier by carrier and interaction by interaction. In $U_{M F}$, no magnetic monopole is needed for these steps (they remain always forbidden by gravity effects on electroweak/electromagnetism) and unfortunately, every GUT implies magnetic monopoles, without exception (See [321] for a discussion) ${ }^{165}$. No GUT (like SO (5), SO (10) and supersymmetric variations) symmetry requirements, could circumvent the ineluctability of the absence of proton decay in inertial frames (at least due to GUTs ${ }^{166}$-there are also other theoretical reasons to expect proton decays that are out of scope of the present paper) [481]) that has never been observed [239].

UU in $U_{M F}$, is the outcome of the quest for the Holy Grail started by Einstein ${ }^{167}$. It takes a different form, and it is for $U_{M F}$. In $U_{M F}$, at $\mathrm{UU}$ ages, regimes or scales, now new particles are needed; other they may all combined into one or few responsible for gravity and all other interactions. It is possible; but not needed at the difference of what was often the conventional image of Grand Unifications.

\subsection{Some of the Baryon Mysteries}

Let us spend a few more moments on the proton, and the problems of the proton decay to see if insights could progress in $U_{M F}$. In fact, can we all but rule out proton decay under certain circumstances (in inertial frames)? Today, the proton decay [239] and the proton radius [500] are still puzzles. [498], show that an (extremal) black hole model for the proton provides a good model for the proton magnetic moment (not depending on the coupling strength), and could explain the different radius estimates as measures of the two Kerr-Newman ergospheres (which depends on $\mathbf{G}$ but gain would have to be adjusted with the massive gravity changes vs. the spin (i.e., going to a Reissner-Nordström metric). Conventionally, conserving all what needs to be conserved 
(energy conserved (energy conservation) is why a (an inertial) proton cannot decay when left on its own into say a positron and a neutron, but it can when it is in nuclei through positron emission (and transformation into a neutron)). In the standard model, there are no other candidates for target result of the proton decay (as long that we have Baryon number B conservation (and Lepton $L)$ ). The details of $B$ and $L$ symmetries and conservations can be found for example in [502]. One note however, that these are anomalous symmetries $[\underline{502}, \underline{504}]$. As a result, they are not associated a massless boson. If $B$ and $L$ could be violated, so that only $B-L$ (Baryon minus Lepton number) needs to be conserved, then proton decay becomes possible. We would encounter New Physics beyond the Standard Model if a boson, associated to the $B-L$. global symmetry [502], was to exist. The associated Feynman diagrams are sketched in [505] (even if in that reference, the lifetime boundary estimates are outdated by far) for the decay to $\pi^{0}$ and $\pi^{+}$. We can see the exotic boson(s) ${ }^{168}$ with fractional charges that differ from the known quarks ${ }^{169}$ ). There is no candidate for these exotic bosons within the Standard Model. They do exist in GUTs, supersymmetry, and variations, and it how these theories predict proton decays as discussed in the previous section. The anomaly that weakens $L$ and $B$ conservation are anomalous symmetries. It results from the fact that interactions involving with left-handed and righthanded massive fermions are not the same [504]. $B-L$ is not anomalous. Gravity and torsion flip chirality of massive fermions [507, 508], and also for massless ones, as already mentioned, or because gravity can flip orientation of spacetime above electroweak symmetry breaking, when everything is massless. Accordingly, gravity flip chirality of massless fermions $[405]^{170}$. As a result, left and right massive fermions, no matter how small their mass, are flipped back and forth when gravity is sufficient, which massive gravity, and small scales, ensure. This could average out the axial current contributions, and eliminate, these anomalies, on average. In other words, we suggest that it may be possible that the anomalies are smeared out by gravity, ensuring truer conservation of $B$ and $L$ than as anomalous symmetries. It is analogous to a similar effect in QCD involving QCD sphalerons [511]. Of course, it is a conjecture, that requires more formal work to validate if such a smearing concept would make sense for anomalies, and what is exactly its impact. All this is for future works. In addition, one should keep in mind the expectation that no global symmetry exists in the presence of gravity [362, $\underline{361]}$ : the smeared, not so much anomalous anymore, symmetries are now local. Yet, it may be an avenue to explain why proton decay is not happening; at least not within any of the scales currently considered. We do not say that it rules it out, because we realize that the story does not stop here, and things can still change at very small scales. Indeed, at very small scales, or very strong gravity levels, combining massless and massive effects, more considerations may enter under consideration. For example, pressuring enough the quarks, gives them full asymptotic freedom with colorless behaviors. When that happens, quarks within a composite could annihilate, and, therefore, proton could decay, and the violations of $B$ (and others) may not matter as the symmetries, (even at decent local scale) would be broken by gravity: this could happen within black holes. But this is a model for macroscopic black holes; not within a stable, or extremal microscopic black hole surrounding particles or spacetime positions.

Besides ruling out the proton decay in inertial frames and at reasonable scales, the implication of eliminating $L$ violation options, with the mechanism proposed here, also has implications for the attempt to explain the neutrino's mass: many of the proposals with Majorana neutrinos for examples are no more viable options.

As carefully stated, the above is a conjecture ${ }^{171}$ in $U_{M F}{ }^{172}$, but it can be mathematically drilled down, and better qualified or proven. Yet mixing in stronger gravity and very small scale may affect all results at all scales. Even if the proposed smearing works, gravity may not let the anomaly reduction be replaced by valid, even local, symmetries for $B$ and $L$. Finally, [503] reviews that $i$ ) anomalies remain meaningful when going to a discrete spacetime (lattice in the case of that paper) and that ii) blackhole radiations can be derived from anomalies.

\subsection{More selected implications of $U_{M F}$ for Quantum Gravity theories, especially superstrings and LGQ}

Let us summarize and detail the properties of a multi-fold universe now that we know that it is actually a fractal/fractional discrete spacetime universe that appears 4$\mathrm{D}$ at larger scales. Gravitons have appeared as spin-2 particles. At discrete (Planck) scales in $U_{M F}$, distances traveled at $c$ speed in $\mathcal{R}_{B G}$ are matched to distances on each fold in the activated folds $F\left(x^{\mu}\right)$ in $B_{\text {activ }}\left(x^{\mu}\right)$. At the initial stages, spacetime $\mathcal{R}_{B G}$ is created in segments determined by perturbations in multiples of minimum time and space jumps with speed maxed by $c$. These are the quanta of spacetime. As activated folds grow, with new segments added, new segments match these particle steps in $\mathcal{R}_{B G}$. For the activated bundle, the torus of $B_{\text {activ }}\left(x^{\mu}\right)$ contributes the same spacetime contribution as in $\mathcal{R}_{B G}:\left(\frac{\delta_{\text {min }}}{2 \pi}\right) 2 \pi=\delta_{\text {min }}$ : gravitons are both the quanta of spacetime in $\mathcal{R}_{B G}$ and the fold creations in $B_{\text {activ }}\left(x^{\mu}\right)$.

With virtual massless particles surrounding physical or virtual particles, massless gravitons are attached to them as the multi-folds and mappings form and grow. Any particle generates them with the virtual particles that it emits.

The concept of attachment is important; it is a new quantum process, although it was already suspected for electromagnetic waves where photons and gravitons travel together, and it links virtual particles to their entangled anti particle. EPR entangled massive particles, and massive virtual particles are associated with massive gravitons. These massive gravitons travel at speeds slower than $c$ and match the speed of the entangled particles to which they are attached. Pairs of gravitons attached to entangled particles are themselves entangled.

The waves of effective potentials and curvatures are the effect in $\mathcal{R}_{B G}$ that, so far, conventional Physics has 
considered as gravitons. They are not, at least in our definition, in $U_{M F}$, or if we want to match our gravitons to those modeled by superstrings. They are more a holographic effect, or reflection of what happens outside spacetime. They are quasi particles and unphysical, while multi-folds are physical. Disentanglement detaches the gravitons from their associated entangled virtual or real particles. Then, the multifolds detach from $\mathcal{R}_{B G}$, and only exists in $\mathbf{A d S}(5)$. That space is tangent dual to $\mathcal{R}_{B G}$ in $U_{M F}$. One could model processes as if when entangled particle are disrupted (e.g. observed or absorbed), so are the correspondingly attached gravitons: they escape and live as a torus or set of (closed) bubbles in AdS(5). It reminds a lot of closed superstrings.

As a result of being in $\mathbf{A d S}$ (5), folds could be $\mathbf{A d S}$ (5) wormholes and even traversable! This may be how our model meets $\mathbf{E R}=\mathbf{E P R}$.

In the emerging spacetime eras, and at very low scales, when spacetime segments are created, microscopic black holeslike dips are added. Each contribute an additional number of degrees of freedom by microscopic area eigen values. The entropy is in $\operatorname{Ln}(A)$. In this regime, spacetime coordinates should be considered as operators. This justifies introducing eigen values for area, or spacetime coordinates. It also clarifies the notion of observable spacetime, and how collapse, or walking on a point, can be understood as concretizing or creating spacetime. In fact, we recover the arguments of $[\underline{208}, \underline{209}]$ about being able to have points and metric operator realized, but without requiring the wave function collapses as in Singh's models. In terms of superstrings, it is probably worth observing the following:

- Gravitons live in $\mathbf{A d S}(5)\left(\times \mathbf{S}^{5}\right)$ or (+1 more for Mtheory); where gravity can be much stronger (as there, gravity is not just an effect tangent to $\left.\mathcal{R}_{B G}\right)$. AdS(5) could be the solutions of $\mathbf{G R}$ for $D=5$ and support strings gravity models.

- Multi-folds could be traversable wormholes. Again, and as already mentioned, we could have modeled multi-fold as ER bridges in $\mathcal{R}_{B G}$.

- Quasi microscopic blockholes surrounding particles could be seen as a start or attachment points from strings characterizing them in $\mathbf{A d S}(5)$. They also motivate a model where each particle are microscopic black holes and spacetime is a network of $\sim$ Planck black holes.

- $\quad$ 94] stated that strings are not compatible with dark energy (positive cosmology constant). If they were to be considered with $U_{M F}$, then it does not matter: superstrings just need to live in $\mathbf{A d S}(5)\left(\times S^{5}\right)$ or $(+1$ more for Mtheory). That incompatibility may also relate to the fundamental instability of AdS as solution of GR [451]. It is not an issue as discussed in the second bullet, below.

- The CFT/AdS correspondence or Gauge/gravity correspondence conjecture is replaced in $U_{M F}$ by $\mathcal{R}_{B G} /$ $\operatorname{AdS}(5)$ correspondence. The arguments for weak gravity effects in $\mathcal{R}_{B G}$ (within $U_{M F}$ ) remain valid (think were graviton live). Yet the correspondence has evolved: gravitons live in $\mathbf{A d S ( 5 ) ; ~ a n d ~ p o w e r ~ t o ~ s u p e r s t r i n g ~}$ theory, or other frameworks, to model them as well as strongly coupled gravity in $\mathbf{A d S}(5)$, because gravitons live there. Of course, we only care about the gravity effects in $\mathcal{R}_{B G}$, where effective potentials or curvatures and their propagation take place as a result of multi-folds and mappings. These are the holographic effects and the CFT/AdS effects for $U_{M F}$. Also, it is no more just a correspondence; we see the boundary/surface effect of the graviton's behavior at the boundary between $U_{M F}$ and $\operatorname{AdS}(5)$ as $V_{\text {eff }}$. No actual gravitons living there can imply a priori weakly coupled gravity.

- $\mathcal{R}_{B G}$ has $\mathbf{A d S}(5)$ as tangent dual space. The outcome is indeed that, instead of having CFT as boundary to $\operatorname{AdS}(5){ }^{173}$. This indicates that superstrings on $\operatorname{AdS}(5) \times$ $S^{5}(+1)$ is where suitable superstrings could exist. It again reinforces the non-issue with non-AdS superstrings being in the swampland [94].

- We know that compacted dimensions as encountered in many string variations for $\mathrm{S}^{5}($ or +1$)$, bring new interactions just as in Kaluza Klein [254, 256]. It would be worth considering if the modelling above shows promises to also look if entanglement gravity-like behavior would be tangent dual space, after all the dynamic multi-folds remind of dynamic compact extra dimensions and dilations in Kaluza Klein and string theory. Yet, it's probably not the case.

- Strings miss the mechanism to well model EPR entanglement, and therefore do not encounter the role of gravitons.

- By not capturing (individual) EPR entanglement, superstring theory did not start as a noncommutative geometry theory. Yet models introducing noncommutativity have been developed, or emerge, in some limits. As an entry point see for example $[\underline{456}, \underline{213}$, 214]. There is a belief that there are deep connections between superstring theories, and noncommutative geometry; but it is not yet well formulated, or understood. In general, in strings, the noncommutativity results from the presence of compactified torus geometry. Our work here shows how it is actually intrinsic to entanglement and gravity with the folds/gravitons attached to entangled virtual or real particles. And yes, our multi-folds are tori in $\operatorname{AdS}(5)$.

- We believe that our model shows how superstrings relate to $\mathcal{R}_{B G}$ in ways that have not been reached yet by string theories because they missed several ingredients, especially in terms of the link to EPR entanglement. Yet the amazing similarities and consistencies are probably points to ponder especially as $U_{M F}$ is not built by simply calculating variations on actions, Hamiltonians or Lagrangian derived from (quantized) Hilbert-Einstein Actions and extensions. Doing so, we may actually provide new insights into M-theory [255, 260]. Consider for example, the fact that strings would apply to spaces outside (i.e., tangent dual to) our spacetime $\mathcal{R}_{B G}$ rather than be the space embedding it. 
Our model has also a lot of connections with aspects of LQG and its numerous variations [243] starting with area quanta with area eigen values and volumes; but that is again because we recover Hilbert Einstein Actions and LQG builds spacetimes or its dynamics from the area invariance interpretation of the Hilbert Einstein Action.

However, these models again do not model diligently entanglement nor gravity as entanglement, nor gravity as entanglement of virtual particles surrounding matter. In fact, many have argued that LQG has challenges modeling matter/other QFT. Spacetime does not exist in LGQ and non-commutativity aspects are inherently introduced for example through the fundamental geometry of the tetrahedron ${ }^{174}$ and non-commutativity of its quanta of area normal vectors. Classical spacetime does not unambiguously emerge in LGQ, and many variations, while discretization and Lorentz invariance are not deducted; they are assumed. With our approach, they could be deducted with reasonings as presented here, where blackholes for spacetime can be replaced by spin networks, started on a random walk fractal structure. It may be worth investigating, along also particles as (larger) black holes or spin networks, with random walks and multi-folds.

Could spin networks or spin foam then be sufficient, or do we also need some quasi-black hole considerations to link back to classical spacetime? We will discuss it in future works. In any case, we believe that matter and entanglement would still have to be separately modeled, which makes sense as in LQG, spacetime does not include matter (fermions and bosons). Matter must be added, and so does entanglement between matter. Details can be found in $[25$, 215]. The same prescriptions apply more or less similarly to most spacetime reconstruction as reviewed in [243], as well as to some extent to models derived from Causal Dynamic Triangulation [459].

Across the board, as well as with superstrings and GR, a challenge is that the Action used by these theories derives from the Hilbert Einstein Action; which we have seen contain a portion of the entanglement effects, yet without surfacing it as a root cause; only a portion (massless). Figuring out how to evolve the Action or models to add what is missing (and avoid duplications) may not be immediate. It is for future works. Some are already in later papers [513]. It would also be worth looking at the idea of adding EPR entanglement, and random walks with its fractional dimensions to LQG and spin networks/spin foam. For future works, it would be great to see if features of $U_{M F}$ can be recovered by the LQG, or one of its variations; although one would have to carefully understand when and where Hilbert Einstein Actions, and its variations should, or should not apply.

Blindly repeating LQG with EPR would at best give a variation mentioned earlier where GR governs aspects of the multi-folds; but it may also rather link LQG to ER=EPR. Yet a lot could be learned from this.

\subsection{From spacetime and particle black holes to Path Integrals and Actions}

Our model of particles and spacetime with microscopic black holes opens the door to another surprising possible link between special relativity and quantum mechanics. We admit that this set of considerations may not be worth much more than other numerological hints. It starts from the work published in [462] where, it is shown that special relativity for supra luminous reference frames implies that in such referential, Physics presents behaviors analogous to quantum mechanics, like probabilistic random behavior and more importantly multiple paths contributing through additive phase contributions to the probability of evolution of particles from a spacetime location to another [463].

Interestingly, if we consider in a microscopic scale (from Planck scale to $\approx f m$ ) world where spacetime and particles are black holes, we could argue that anything appearing, approaching or interacting at such scales behaves with a slowed down time, as times slows down near a black hole horizon.

From that point of view, the quantum and macroscopic world operates much faster and maybe give the illusion to behave (quasi) like supra luminous reference frames (knowing full well that the speed of light remained the same across all these scales, this is why the arguments are for sure not rigorous). But on that basis, Quantum Mechanics and Path Integrals as well as Action extremization would be natural consequences. Regarding our earlier discussion filtering out space like paths in Path Integrals in $U_{M F}$, we already know that the discrete spacetime explains that observation and recipe.

Yet, in the context of the proposal discussed in this section, paths around a microscopic black hole always appear supra luminous in a larger scale supra luminous frame. It means that if this was a way to explain the origin of Path Integrals and Actions extremization, then indeed no path outside the light cone is to be considered. This is what we had already settled upon in section 2 .

\section{COSMOLOGY, BIG BANG AND ALL THESE DARK THINGS}

\subsection{The Big Bang and Inflation}

In the case of our multi-fold universe $U_{M F}$, a big bang initial expansion $^{175}$ of the universe can be understood as a (re) construction of $U_{M F}$ starting from single point or a small set of point. Quantum fluctuations initiate random walks of the particles that are created. For simplicity, we assume that the typical big bang chronology [167] is respected. Therefore, some unification of the forces probably exists, at least up to electroweak but possibly as UU. Because entanglement is so central to quantum physics, we do expect that even a unification of gravity with other interactions would maintain the behavior described in this paper in terms of multi-folds. The main difference is that initially all particles were most probably massless, EPR entanglement at these levels of energy and scales is also with massless gravitons: indeed, all physical or virtual particles are massless. Cosmology 
considerations and the Standard model can describe the chronology and zoology of particles (and their field model) that were present and involved. It may be some of those that we know today. Or it may new ones, including say inflatons $[\underline{455}, 453]$, gravitons, dilatons, scalar fields, etc. It does not really matter for what we try model. Yet it is interesting that we do not need to introduce an inflaton as will be discussed later on: it can be, or not be, among these early particles. In fact, inflation itself can directly be explained with the reconstruction mechanism that we described and the random walk.

Indeed, at every time click, and with large energy content at every concretized point of spacetime, each point will contribute multiple new points in many directions (by populating with a new particle). So, even starting from a single point ${ }^{176}$, we have an exponential growth of new spacetime points with time, which is inflation. A phase transition occurs when the energy at each point does not automatically so enthusiastically create new points in every direction, but it rather follows a random walk and exponential growth stops.

The random walk is, at times, mostly between concretized points, and, in some places, it is not: exponential growth stops. Expansion however continues as will be revisited later, through random walk, possibly slowed down by entanglement with new/recently visited spacetime points. Reheating etc. can take place, maybe as the result of other cosmology and standard model considerations mentioned, like UU symmetry breaking, then GUT symmetry breaking if it were to exist (doubtful as discussed), and then, for sure, the Electroweak symmetry breaking for sure, etc.

Of course, suitable conditions were needed for the big bang to start from an initial point/seed or region (possibly large) requires enough energy within the initial seed volume or initial fluctuation; something that obviously would be extraordinarily rare, if seen as a vacuum fluctuation. If lots of energy is present e.g. if it was happening at the end of a crunch cycle like if we had a big bounce as proposed, and discussed, in [457], then it may immediately start at the first fluctuation. Otherwise, we do not know what initiated the process, just as we also still do not know in the case of all the other conventional models for cosmology. Note that this may also be seen as having missed the singularity, just as a particle might see a black hole as a wormhole if that were to happen thanks say to rotation of the black holes (or again torsion, spacetime discreteness etc.).

\subsection{Dark Energy and the Cosmological Constant problem}

Quantum walk continues to generate new spacetime, but typically the effect is constant (or decreasing where spacetime is already extensively realized, i.e., concretized). This explains a constant or decreasing expansion (e.g. with a static or slowly decreasing cosmological constant).

This effect is not primarily due to the vacuum energy. So, in our model, and multi-fold universe, we can resolve the cosmological constant problem, whereby there is a difference of $10^{60}$ to $10^{120}$ orders of magnitude of difference between the vacuum ground energy (due to vacuum fluctuations) predicted by QFT/Standard Model and variations believed to be the source of the cosmological constant versus the actual value estimated of the cosmological constant $[\underline{219}, \underline{474}]$. These subtleties and the mechanisms that we describe may also help understand the discrepancies between the measured cosmological values versus QFT prediction so far based on the vacuum energy [219], and possibly address the "cosmological constant paradox" (aka " $\Lambda$-paradox").

With our mechanisms, it is not surprising that the value of the cosmological constant would be small, no matter what the QFT vacuum energy density is: it solely intervenes to create the expanding displacements. These are expected to be of orders of magnitude smaller than Planck length $\left(l_{P}\right)$, and hence really small.

A lot of them spurred by a lot of energy density is required to have any macroscopic let alone cosmological effect. As time goes by, spacetime entanglement relaxes. Even with all spacetime points concretized through different random walks, fluctuations of the position of these points may generate effective potentials that attract outside of the concretized spacetime as illustrated in Figure 15.

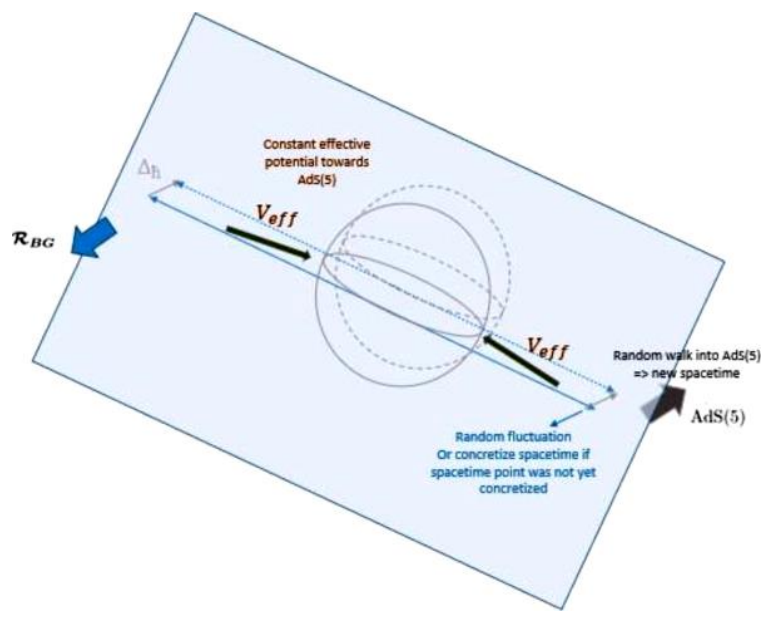

Figure 15: Fluctuations of the position of the entangled particles, result into the attractive effective potential to point towards the tangent dual AdS(5) space and produce a (constant) force to grow spacetime and hence accelerate expansion. Fluctuations within spacetime only introduce torsion, or fluctuations in spacetime, or concretize not yet existing spacetime as constant rate processes (lattice points never occupied vs. in the first case where new lattice points are created in an accelerated way). The figure illustrates symbolically the 4-D spacetime.

The random fluctuations of the particles amount to random walks to grow spacetime constantly. However, the continued effective potential is an extra pressure, that can be modeled as a constant gravitational pressure in GR. Even if very small, it eventually ends up accelerating constantly the growth of the universe, in all directions, everywhere. When a fluctuation rather brings the attraction effect towards the existing spacetime, we have noise in the attractive effective 
potential; but not the same impact on the expansion of spacetime. It is more random walk effects.

The combination of these effects (relaxation of spacetime entanglement, random walk, and fluctuations that create an accelerating attraction towards $\mathbf{A d S}(5))$ results into accelerated expansion of $\mathcal{R}_{B G}$, not just a constant expansion. The attractive potential effect accelerates that expansion. This contribution can explain aspects of dark energy, especially in terms of accelerated expansions. It is a direct result of the multi-fold mechanisms of $U_{M F}$.

Indeed, dark energy [ $[\underline{310}, \underline{216}]$ was introduced to explain the observations of the universe expansion is currently accelerating instead as decelerating as was originally expected. Its proportion is modelled as part of $\Lambda$ CDM [217, 218], along with dark matter. In our proposed multi-fold universe $U_{M F}$, acceleration of the spacetime expansion is automatically expected: everywhere, physical or virtual particles are at the local edge of spacetime. Just as for the kickoff of the big bang, whenever fluctuations, or uncertainties, push the attraction target outside spacetime, spacetime grows with the resulting attraction forces: it is a perpetual acceleration; without introducing new particles or repulsive gravity.

And again, it looks like; the cosmological constant is not directly a measure of the vacuum energy but rather the result of uncertainties, associated to it, that pull everywhere particles (real and virtual) outside spacetime.

Accelerated expansion and the positive cosmological constant can result from these effects. As in traditional models, and $\Lambda$ CDM $[216,217]$, it is widely expected that the density of the dark matter is uniform throughout spacetime, and remaining constant as it expands. In our multi-fold universe, it may not be the case: the dark energy phenomenon is uniformly happening everywhere; yet, because it results from fluctuations, it is expected to be more pronounced near "hot objects" and so near matter/energy. Also, random walks expansions outside spacetime is more pronounced where matter is than in only vacuum, and therefore where there are only virtual particles. From a larger scale point of view, matter curves spacetime more near large, massive objects [220] (positive curvature and elongated geodesics, even if mostly with time dilation) and fluctuations will more often be attracted towards the outside of the spacetime (at least where the effect is convex); which is always the case if we started from flat $\mathcal{R}_{B G}$. In a multi-fold universe $U_{M F}$, the accelerated expansion of the spacetime results from the fluctuations and uncertainties of the position of particles. The effects are probably more pronounced around matter and energy, and so dark energy is probably not uniform but stronger around matter. It has been envisaged in the past; see from example [221, 222] for some examples of possible modelling and implications; but our proposed behavior may not be as envisaged in these papers.

Finally, matter/energy enhanced dark energy behavior would also prevent singularities, and encourage cosmology dynamics, like big bounces (in addition to the effects of torsion and discrete spacetime).

\subsection{Dark matter}

Dark matter $[\underline{217}, \underline{223}, \underline{310]}$ is the other key pillar to $\Lambda \mathrm{CDM}$ $[\underline{216}, \underline{217}]$. Motivations for its introduction are detailed in $[217, \underline{223}, \underline{310]}$. To this day it has remained unexplained even if candidates have been proposed like Scalar Field BEC (e.g. of massive axions, gravitons, neutrinos or Higgs field etc.). It is argued that the mass deficit in the universe rather comes from cold dark matter (i.e., invisible matter moving slowly); in order to explain the large structure of galaxies and galaxy clusters in the universe that are believed to not be possible if dark matter was moving rapidly.

In our proposed multi-fold universe $U_{M F}$, a new candidate emerges in the form of the long-distance entanglement (but mostly not as proposed, so far, in say [14]). Per section 4.1, entanglement between two or more particles creates an attractive potential in $(1 / r)^{177}$.) towards the center of mass on the axis between the particles. If particles like photons, and more importantly neutrinos are generated by matter (e.g. nuclear reactions in stars), then they lead to two or more particles entangled (e.g. by spin, polarization/helicity, states etc.). In the case of neutrinos, the center the mass remains near the source. With photons, the center of mass of entangled photons emitted in opposite directions is also staying near the source in a spherical halo. As the particles travel, they create additional attraction towards these centers. Even more if they interact and entangle with other particles without losing the original entanglement.

Particles that interact barely like neutrinos have increased chance to maintain long time entanglement and hence emulate as if additional dark matter (dark and cold as the center of attraction is not moving fast-and yet it is nonexisting, hence never (so far) discovered, no matter what) was present.

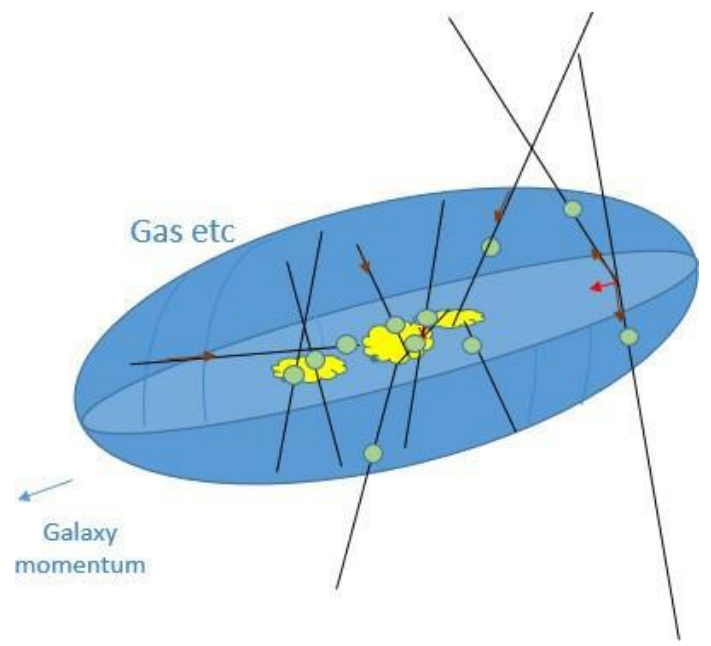

Figure 16: Entanglement from matter or between emitted entangled pairs of particles creates impressions of attraction towards the galaxy or halos of the galaxies. If not, enough matter is kept entangled in or towards galaxy (or if not, enough halo exists to capture entanglement with the center, the effect may decrease or become negligible. 
In addition, as these particles randomly walk on their path, they create spacetime entanglement on their paths. These also contribute to attractive potentials. This way, most scenarios requiring dark matter might be accounted for [223]. The distributed center of attractions where sources are and in a diffused or halo region surrounding the galaxies as observed. When due to the history of the galaxy, entanglement is weak, disturbed, or has been dispersed, and spread further away (e.g. on the path of movement of the galaxy), it may appear as if less or no dark matter is associated to a galaxy. This could avoid the challenges of observations like [458].

\section{VALIDATION AND EXPERIMENTATION}

So far, our approach has been to work in $U \in\left\{U_{M F}\right\}$ associated to a pseudo-Riemannian manifold $\mathcal{R}_{B G}$, without claiming that such a model is relevant to $U_{\text {real }}$, and being content to explain or relate the model to what has been modeled, or observed, so far by conventional physics in $U_{\text {real }}$. Of course, it would be even better if we could discover or prove that $U_{\text {real }} \in\left\{U_{M F}\right\}$.

Our multi-fold universe $U_{M F}$ differs from the universe modelled so far by conventional physics by numerous aspects ranging from the concepts of multi-folds, and mappings, the absolute respect of $c$ as upper speed limit for particles (physical or virtual), and any communications, exchanges, or interactions, the filtered Path Integrals $\mathcal{P} \boldsymbol{I}_{S F}$, the kinetics and dynamics of bunches of multi-folds, associated to activation, and deactivation, events, the resulting attractive effective potentials due to entanglement, and the associated (effective) curvature of the multi-folds as well as a plausible discrete and fractional dimensions for spacetime, the presence of $\mathbf{A d S}(5)$ spacetime tangent dual to spacetime around every physical or virtual particles, the attractive effective potential in $(1 / r)$ (and possibly variations) as well as the direction privileged gravity-like attractive potentials between non hierarchically EPR entangled particles, (quasi) microscopic black hole around particles and as spacetime, virtual particles around virtual, or real particles, carrying gravity, massless and massive; the graviton attachment to EPR entangled particles in all these phenomena, the Higgs and the graviton roles in random walks, and entanglement of spacetime quanta, the potential impact of the models on the big bang inflation, dark energy, cosmological constant, and dark matter, and entangled quantum matter, and the generalization of Area laws, and holographic principles; the role of gravity as extension to the Standard Model, without the need of new Physics; the absence of a strictly weak gravity; new lifecycle for black holes and the Holy Grail of a Ultimate Unification. These points also resulted in recommendations and considerations for other Physics theories.

Being able to model theoretical variations that can be verified or predictions that are validated or invalidated would open such possibilities.
Possibly, the most obvious phenomena that could be observed to validate the approach would be the attractive effective fluctuations in $(1 / r)$ (gravity like) near/within entangled systems, especially in the case of macroscopic entanglement (assuming that they are not hierarchical). These effects do not range to infinity and they propagate as waves or fluctuations when entanglement takes place or collapse. So, one should observe mostly gravity fluctuations within or around entangled quantum matter like superconductors.

In particular, it would be valuable to try to measure such fluctuations, especially around high temperature superconductors, where we expect the effect to be stronger (because superconductivity seems to be originating from tighter pairs than conventional superconductors, even if the pairs are spread also across the superconductor, and assuming the roots of the entanglements are not hierarchical. Besides this, in the short term, entanglement-based dark matter seems our best bet, and way forward.

We already noted that related observations may have taken place but have been plagued with controversies, and conspiracy theories ranging from graviton generators to antigravity with repulsive gravitons; something that our model does not propose. Yet, to the extent that it would credible, and that we manage to understand the details, the works described in $[\underline{224}, \underline{225}, \underline{226}]$ may have shown that some High Temperature superconductors, when rotated, generate a gravity field consistent with linearized gravity field equations predictions (Einstein Maxwell equations for gravity) [227, 228, 229], and may relate to our predictions in a multi-fold universe: the observed perturbations of accelerometers are consistent with attractive gravity contributions, and the order of magnitude discrepancy (stronger than expected) could result from attributing the effect the entanglement gravity-like fluctuations, and massive gravitons, as we predict. More experiments aimed at detecting gravity-like fluctuations within and around superconductors and other entangled quantum material, or macroscopic entanglement, are encouraged. Pure experimentations not involving electromagnetism would provide clearer answers, but it is possible that electromagnetism is needed to attain observable effects.

Qubits realizations (non-hierarchical or even hierarchical where forces compose) may also be a basis for observing gravity fluctuations. We know that EPR entanglement is typically not observable [87]. Detecting gravity like fluctuations between Qubits is certainly a way to determine if systems are entangled, and form a Qubit without perturbating the Qubit (i.e., by measuring it). However, effects are way too weak for Today's measurement, or detection, capabilities. Yet there may be indirect ways to detect the effect. As quantum computers are built, it is expected that they will concentrate large numbers of Qubits in controlled geometric configurations. This may provide a way to detect gravity like fluctuations.

This link between quantum computing and gravity due to entanglement has of course other interesting considerations. Indeed, for example, we know that the AdS/CFT correspondence conjecture led to the observation that 
spacetime $\mathcal{R}_{B G}$ (or rather a CFT spacetime) may behave as an error corrector code for Qubits/entanglement in $\mathbf{A d S}(5)$ [233, 234, 235]. However, these observations apply to Qubits realized in $\mathbf{A d S}(5)$; not in $\mathcal{R}_{B G}$. By analogy of the tensor networks, and its renormalization group, behind the error correctors, and the evolution of entropy from $\ln (A)$ to $A$ that we observe as we grow in scale, we can see that in a $\mathcal{R}_{B G}$ of $U_{M F}$, the bulk discrete structure of spacetime is also encoded with error correction codes in a larger scale surface surrounding it. In fact, it explains all the Area properties and laws seen so far. This statement is actually a fundamental new theorem, and not something that has been clearly demonstrated so far, in our view. It is a fundamental link to unitarity expectations in quantum physics. More on this in future work.

\section{PUTTING IT ALL TOGETHER: FROM SCIENCE TO APPLICATIONS AND SCIENCE-FICTION}

\subsection{Hilbert spaces, Rigged Hilbert Spaces, Fock Spaces and $U_{M F}$ spacetime}

With $U_{M F}$, we have learned that one can look at state spaces like Hilbert spaces, and their rigged (e.g., Gelfand triplets), or Fock versions, for QFT, where wave functions and fields live, are not just built on the spacetime of $x^{\mu}$, but impact it. Indeed, we saw a few examples and mechanisms:

- We have seen that EPR entanglements between points or regions (at least nonhierarchical) result into folds and mappings between these regions. The multi-folds are tangent dual to spacetime in the center of mass of the entangled particles. Similar conclusions aligned with our view was also reached independently in [190]. We also saw how this relates to the phase space [246].

- We also saw that, when considering histories from $t=$ $-\infty$, spacetime points that have not been crossed by a particle, do not exist. They appear only if the wavefunction was not zero at some time $t_{1} \in(-\infty, t[$. Otherwise spacetime is created only at $t_{2}$ when the wave function becomes nonzero. These ideas are also consistent with proposal related to wave function collapses (which are not or proposal here) in [208, 209]: the concept of generating spacetime this way is analogous. Somehow, wave functions appear real, beables.

- Uncertainty on the wavefunction can not only contribute to the above, but also dilate the mapping of the Hilbert space to the configuration space to account for new intermediate spacetime points.

- Spin might even be a reflection of multi-folds.

If we think about it, this relationship is not that surprising, and it is not just related to our model. If indeed, as many believe by now, entanglement (or information or entropy) impacts spacetime, then we have challenge with most conventional Quantum Physics, when the wave function is not considered a real, a beable. So, it is either a beable, that can have such an impact through physical interactions with spacetime; or spacetime and Hilbert spaces must have a physical relationship.

This relationship, between the state space/Hilbert space(s), and the configuration space structure (i.e., spacetime) in $U_{M F}$, certainly warrants further analysis and formalism. The wavefunction (of the universe content) defines in the right Hilbert spaces (or variations) creates or concretizes spacetime (where there was none) and creates multi-folds, and effective potentials and curvatures (where spacetime exists). Said differently, the spacetime wavefunction is defined by the Hilbert spaces properties of its content, and its topology (or energy content via its effective potentials) changes as a result. A whole new formalism could be derived from this observation, and it may help with some of the wave functions paradoxes and all the Quantum Mechanics interpretation disagreements. It is for future work. Some ideas can already be found among the papers at [513].

\subsection{Applications and Engineering dreams}

If $U_{M F}$ correctly describes $U_{\text {real }}$, then, while it is very difficult to foresee, at this stage, all what could result from our multi-fold universe model, besides impact on Physics, we can envisage applications in terms of:

- Detecting entanglement without perturbating the entangled systems by detecting gravity fluctuation (the attractive effective potential) between, or around, the entangled components.

- Tuning gravity locally by playing with entanglements.

- Polarizing the vacuum to modify gravity locally. Entanglements and polarization of vacuum may be such that entangled virtual particles generate additional $V_{\text {eff }}$ attractive in directions prescribed by the polarization. This may also be an avenue for verification of our theory.

- The relationship between Qubits and quantum spacetime, opens the door to a myriad of applications for quantum computing; that it be in terms of design of robust quantum computing systems, and quantum algorithms, especially around quantum error correcting codes for computing, storage and communications. As well as using more natural systems (spacetime) as computing resources.

- These applications combine directions for applying the principles of our models as well as achieving validation or falsifiability.

\subsection{Science-Fiction and Everybody's Non-Sense}

There is no lack of science fiction, or futuristic avenues that could potentially be explored. But the present models have opened the door to the following considerations: 
- Teleportation beyond the EPR Quantum teleportation [109], and communications mentioned earlier: if one was able to i) activate a fold (e.g. in $\mathbf{A d S}$ (5) where wormholes could be traversable ii) exchange matter or information in a fold while observing one of entangled particle (or on the support domain of the mapping); then it might be possible to transport information immediately ${ }^{178}$ from the location of an entangled particle in $\mathcal{R}_{B G}$ to the location of its entangled correspondent in $\mathcal{R}_{B G}$. If, in $\mathbf{A d S}(5)$, bundles of folds are not (necessarily) wormholes or black holes then we may not have to resolve the same travers ability problems. If one could (re)use an existing activated fold between entangled particles then sending matter, or information, while observing the particle could send it to its entangled companion, which may be at the other edge of the universe! It is important again to note that many GR models prevent travers ability, or communications [50], yet there are theoretical possibilities even for them [230], but in such cases traversing them means covering distance larger than between the "mouths". Unfortunately, activation on demand, reuse of activated folds, grouping to macroscopic scales, and navigation through it is totally undefined at this stage and probably makes no sense. Yet, with $U_{M F}$ the physics in $\mathbf{A d S}(5)$ may also not have to be limited to GR.

- EPR based navigation at quasi speed of light: ignoring the major challenges that the above is something we do not know how to achieve, or if even possible, one could imagine a system that emits a pair of EPR entangled particles, massless particles (e.g. photons) to reach $c$, and captures one (think for example of an optical trap with optic fibers and mirrors). It is then followed by the observation of the "captured" photon, which might allow to use the approach of the previous bullet to travel (i.e., send matter or information) to the place reached by the other photon.

- Capturing entangled (massless) particles from somewhere could similarly enable communications, or travel to its entangled partner... Of course, that would rather be a random guess as to where what we send would end up.

- Other wormhole and black hole exploitation targeting the microscopic (quasi) black hole surrounding every particle and the associated tangent dual AdS(5) spaces. The bottom line is that entanglement activates multifolds and folds could be treated like portals to $\mathbf{A d S}(5)$. For example, folds and $\mathbf{A d S}(5)$ may expose wormholes as discussed above, or even closed time like trajectories (time travel) in AdS(5). But again we do not know how to interact with the folds and even less how the tangent dual AdS(5) space is "really physical". But yes, in $\operatorname{AdS}(5)$, with GR, time travel may be possible; see for example [231]. . .
- Vacuum polarization to create gravity like fluctuation, may allow to, we cringe to say it, reduce gravity felt by an object. . . yes an antigravity mechanism.

Now that these crazy baseless ideas have hit all the craziest possible topics ${ }^{179}$, and may have made the paper even more questionable to many readers ${ }^{180}$, it is probably time to conclude. Yet again, all this is ok in $U_{M F}$, if it is not $U_{\text {real }}$. Criticizing these considerations can only be because the reader already starts to consider the plausibility that $U_{M F}$ may model $U_{\text {real }}$ well. Then again, maybe it is worth considering [517].

\section{DISCUSSIONS AND CONCLUSIONS}

Let us summarize concretely what we have done and learned in this paper. The fundamental ideas and principles are the introduction of a universe where nothing can go faster than the speed of light. As a result, we had to find a mechanism to handle Einstein's spooky action at distance encountered in EPR entanglement. Inspired by the principles of Path Integrals, we proposed a mechanism of multi-folds, and mappings from the background spacetime, activated by events of EPR entanglement of real and virtual particles, and deactivated when particles are disentangled. With this model, the resulting activated multi-folds create attractive effective potentials towards the source of the particles (center of mass of EPR entangled particle or particle emitting massless and massive entangled virtual particles that can be carriers of interactions). These effects can also be seen as contributing at every point of spacetime an effective curvature (Ricci scalar), and direction (Ricci Tensor). The resulting effective potential, felt in the background spacetime, behaves and propagates in spacetime, as a spin-2 perturbation of spacetime, that can be massless (at $c$ ) or massive (propagating a speed lower than $c$ ). The model is new for EPR entanglement where we predict gravity-like fluctuations towards the center of mass of entangled particles. When considering the entangled virtual massless particles emitted near a source of energy, we recover a gravity like behavior.

The effects amount at larger scales to defining a field of curved manifold on the background space that follows Einstein's GR field equations, and Newton gravity in linear approximations (and as a result, gravity's area laws). Virtual massive particles add at very small scale multi massive gravity contributions. The multi-folds attached to the entangled particles live outside spacetime: they are the spin2 gravitons when quantized living in $\mathbf{A d S}(5)$. Our model is covariant, and background independent. Its approach, with respect to a background spacetime, avoids the problems of divergences and non-renormalization. This new model for gravity and recovery of classical results is remarkable considering that only the requirement of no supra luminosity was imposed, and as a result we proposed a multi-fold mechanism to address the paradoxes of EPR entanglement, and its spooky action at distance. It is remarkable that gravity emerges from entanglement between virtual particles, and 
additional gravity-like fluctuations appear between entangled systems in general: no introduction of HilbertEinstein Action, or derived variations, or area invariance were used to reach these results. We note the irony that gravity, and GR are recovered from entanglement, despite usual expectation that these theories would be incompatible: one can hardly find a more quantum characteristics than entanglement... The activated multi-folds implies that gravitons live in an anti-commutative $\mathbf{A d S}(5)$ spacetime tangent dual to $U_{M F}$ at the attraction point of the effective potential for a given entanglement and tangent dual to $U_{M F}$ at the position of every particles (and concretized spacetime) when looking at its gravity effects. Every particle is surrounded by a microscopic black hole. This leads to a recovery of a holographic principle and a new interpretation of the AdS/CFT correspondence in $U_{M F}$. Our model seems to qualify, with twists, the pictures painted by other approaches like superstrings. It even explains why they would live in AdS spaces, while our universe is not of this type, and why the positive curvature (and cosmology constant) incompatibilities with superstrings may not matter. These results also inspired the next steps of our work where we showed that spacetime is discrete with a noncommutative (and non-associative) geometry that maintains Lorentz invariance. At very small scales (Planck scales) can be modeled as a network of Planck scale Schwarzschild microscopic black holes, where the nodes form a fractal generated by random walk (footprints of black holes where particles passed before), and particles are themselves charged black holes (extremal with spin rotation handled through the multi-folds). The model again recovers Einstein's GR field equations. It also explains why at the smallest scales, spacetime appears as 2-D, while gravity, and spacetime are 4-D at larger scales. Quantizing spacetime associates gravitons to quanta of multi-folds and matching spacetime changes, when spacetime is created or perturbed. They live in $\mathbf{A d S}(5)$, and have an effect in our spacetime through the effective attractive potential fluctuations, that result from their association to entangled virtual or physical particles. The combination of spacetime associated to the microscopic black holes and the discrete graph implies that semi-classical models can be used up to very small scales. The combination of discrete spacetime and compatibility with microscopic torsion as well as dark energy mechanisms of the multi-folds, guarantees the absence of gravity related singularities (e.g. in black holes, or in cosmology), and can support big bounce scenarios. The approach that we propose illustrates the impact of adding gravity to the Standard Model, and how many open issues in Physics may get handled as a result. We also used it to build contributions to address open issues with cosmological inflation, dark energy, cosmological constant and dark matter. Finally, the underlying entanglement model opens opportunities to validate our model in cases of entangled systems like superconductors or detect entanglement without disturbing the system. Even more fanciful uses cases have been introduced. There are many other key nuggets in the text, left to the reader to notice.
At the end, we were very surprised by what we found out. Many unexpected things, even if possibly present in other approaches, popped up in our model. But ultimately, the biggest surprise was how analyzing the discrepancy between our approach, and the weak gravity conjecture, led us to discover new life cycle options for Black hole evaporation and ... glimpses of the Holy Grail: The Ultimate Unification UU. The latter was Einstein's dream and has occupied many bright mind since. It was totally unexpected that we would find that gravity, and the other interactions would meet in strength at very small scales, and that in fact all interactions would also become equivalent carriers of gravity, (and still their charges) which is the ultimate symmetry and justification.

A lot of work is still needed. In particular to move to more quantitative expressions, detailed experiments and applications of interest and to further pursue the impact of $U_{M F}$ and gravity on the Standard Model and Physics in general. We also believe that we have discussed interesting implications for related and competing models that will warrant exploring if these insights help or not with these approaches. With all the results we have, including with respect to the Standard Model, UU, black hole entropy, entropy in general, etc., we believe that it is now possible to start tracking if some coupling constants and other parameters can be quantified and added to the model; while awaiting validation, which may take a while considering the weakness of gravity at our scales. And, yes, we believe that $U_{M F}$ has many interesting characteristics that make it a good candidate to model $U_{\text {real }}$. Look at $[517]$ for more.

\section{FINAL NOTES}

Cite as: Stephane H. Maes, (2020-2022) "Quantum Gravity Emergence from Entanglement in a Multi-Fold Universe", HIJ, Vol 2, No 4, pp 136-219, Dec 2022,

https://doi.org/10.55672/hij2022pp136-219,

https://shmaesphysics.wordpress.com/2020/06/09/paperpublished-as-preprint-quantum-gravity-emergence-fromentanglement-in-a-multi-fold-universe/, and viXra:2006.0088, (June 9, 2020). [HTML]

Updates, corrections and many papers detail the Multi-fold theory and providing new results can be found at [513] (and discussions); in particular at $[\underline{514}, \underline{515}, \underline{516}]$. Also, some 2022 papers include key results indicating that the real universe may indeed be multi-fold. The most notable is probably [517]

\section{ACKNOWLEDGMENTS}

The author thanks Corrine, Alexander and Christopher Maes, for their love and patience, and acknowledges their help with reviewing the paper. He also acknowledges his corporate employers (Micro Focus then IFS) for enabling him to pursue this work in his past time, and his colleagues for stimulating discussions. To Karen A., with Love. 
This paper would not have happened without stepping on the shoulders of giants who created, explained and taught us all what was used. We hope they will forgive all mistakes, and omissions.

\section{REFERENCES}

[1] W.M. Keck Observatory, (2019, October 23). "A crisis in cosmology: New data suggests the universe expanding more rapidly than believed", https://phys.org/news/2019-10-crisis-cosmologyuniverse-rapidly-believed.html, Retrieved October 23, 2019.

[2] Rovelli, C. (2008), "Quantum gravity", Scholarpedia, 3(5):

7117 , http://www.scholarpedia.org/article/Quantum_gravit y, Retrieved March 2, 2019.

[3] B. Clegg (2019), "Dark Matter and Dark Energy: The Hidden $95 \%$ of the Universe", Icon Books Ltd

[4] Einstein, A; B Podolsky; N Rosen (1935-05-15). "Can Quantum-Mechanical Description of Physical Reality be Considered Complete?", Physical Review. 47(10): 777-780.

[5] Bell, John. "On the Einstein-Poldolsky-Rosen paradox", Physics 1 3, 195-200, Nov. 1964.

[6] Isaac Chuang and Michael Nielsen, (2000), "Quantum Computation and Quantum Information", Cambridge University Press, October 2000.

[7] Richard Feynman, (1996), "Feynman Lectures On Computation", Edited by H.G. Hey and R. W. Allen, Addison-Wesley Publishing Company.

[8] Christopher M. Hirata (April 2017). "Lecture xxxIII: Lagrangian formulation of GR", http://www.tapir.caltech.edu/ chirata/ph236/201112/lec33.pdf. Retrieved March 2, 2019.

[9] Edmund Bertschinger, (2000), "Symmetry Transformations, the Einstein-Hilbert Action, and Gauge http://web.mit.edu/edbert/GR/gr5.pdf. Retrieved March 2, 2019.

[10] Erik P. Verlinde (2010), "On the Origin of Gravity and the Laws of Newton", https://arxiv.org/pdf/1001.0785.

[11] Paul Ratner, (Nov, 2016), "Remarkable New Theory Says There's No Gravity, No Dark Matter, and Einstein Was Wrong", https://bigthink.com/paulratner/remarkable-new-theory-says-theres-nogravity-nqo-dark-matter-and-einstein-was-wrong, Retrieved on November 27, 2016.
Correspondence and requests for materials should be addressed to Stéphane H. Maes (email: shmaes.physics@gmail.com).
[12] Chris Lee (May, 2017), "Diving deep into the world of emergent gravity", https://arstechnica.com/features/2017/05/emergentgr avityanddarkmatterexplainedbyexciteduniverse/, Retrieved May 23, 2017.

[13] Sabine Hossenfelder (Feb, 2017), "Recent Claims Invalid: Emergent Gravity Might Deliver A Universe Without Dark Matter", https://www.forbes.com/sites/startswithabang/2017/ 02/28/is-dark-matter-about-to-be-killed-byemergent-gravity. Retrieved on November 1, 2018.

[14] Erik Verlinde, (2016), "Emergent Gravity and the Dark Universe", https://arxiv.org/pdf/1611.02269v2.

[15] Eliano Pessa (2009), "The concept of particle in Quantum Field theory", https://arxiv.org/pdf/0907.0178v1.

[16] "Lecture 1: Introduction to QFT and Second Quantization",

https://rdmc.nottingham.ac.uk/bitstream/handle/inter nal/102/Quantum\%20Field\%20Theory/output1.pdf, Retrieved on Oct. 27, 2019.

[17] P.J.H. Denteneer, 2008. "Second Quantization. Lecture notes with course Quantum Theory", http://wwwhome.lorentz.leidenuniv.nl/pjhdent/SecQuant08.pdf, Retrieved on Oct. 27, 2019.

[18] Ted Jacobson, (1995), "Thermodynamics of Spacetime: The Einstein Equation of State", https://arxiv.org/pdf/gr-qc/9504004v2.

[19] de Haro, Sebastian; Dieks, Dennis; 't Hooft, Gerard; Verlinde, Erik (2013). "Forty Years of String Theory Reflecting on the Foundations". Foundations of Physics. 43 (1): 1-7.

[20] Greene, Brian (2000). "The Elegant Universe: Superstrings, Hidden Dimensions, and the Quest for the Ultimate Theory". Random House. ISBN 978-09650888-0-0

[21] "Quantum Gravity", Wikipedia, https://en.wikipedia.org/wiki/Quantum gravity. Retrieved March 2, 2019.

[22] R. Loll (2019), "Quantum Gravity from Causal Dynamical Triangulations: A Review", https://arxiv.org/pdf/1905.08669v1. 
[23] H. W. Hamber, 2009, "Quantum Gravitation. The Feynman Path Integral Approach", Springer.

[24] Hrvoje Nikoli, (2013). "Relativistic Quantum Mechanics and Quantum Field Theory". https://arxiv.org/pdf/1205.1992v1

[25] Rovelli, Carlo (2004). "Quantum Gravity". Cambridge University Press.

[26] Malament, D.B. (1996). "In defense of dogma: Why there cannot be a relativistic quantum mechanics of (localizable) particles". In Clifton, R.K. (Ed.). "Perspectives on quantum reality" (pp. 1-10). Kluwer: Dordrecht.

[27] Daniel Cavalcanti Santos, (2008). "Entanglement: from its mathematical description to its experimental observation", PhD Thesis, Universitat de Barcelona, https://www.icfo.eu/images/publications/DT 0801. pdf. Retrieved on March 4, 2019.

[28] J. M. Maldacena, "Black Holes and Holography in String Theory". In: B. Duplantier and V. Rivasseau (Eds): "Seminaire Poincare", 61-67. (2004).

[29] J.M. Maldacena, "The Large N Limit Of Superconformal Field Theories And Supergravity", Adv. Theor. Math. Phys. 2 (1998) 231

[30] Muxin Han and Ling Yan Hung. (2017), "Loop quantum gravity, exact holographic mapping, and holographic entanglement entropy". Phys. Rev.d, 95(2), 2017

[31] Y. Jack Ng, (2016), "Holographic Theory of Gravity and

Cosmology", https://arxiv.org/pdf/1610.06236v1.

[32] Ted Jacobson and Renaud Parentani (2003), "Horizon Entropy", https://arxiv.org/pdf/gr-qc/0302099v1.

[33] Ted Jacobson, (1999), "On the Nature of Black Hole Entropy", https://arxiv.org/pdf/gr-qc/9908031v2.

[34] T.Padmanabhan, (2010), "Thermodynamical Aspects of Gravity: New insights", https://arxiv.org/pdf/0911.5004v2.

[35] "Summary of the Standard Model", http://bolvan.ph.utexas.edu/vadim/Classes/11f/SM.pdf. Retrieved March 2, 2019

[36] Wikipedia, "Mathematical formulation of the Standard Model", https://en.wikipedia.org/wiki/Mathematica formulati on of the Standard Model. Retrieved March 2, 2019.

[37] "Standard Model", Wikipedia, https://en.wikipedia.org/wiki/Standard_Model. Retrieved March 2, 2019.

[38] A. Hebecker and J. Hisano (2016). "Grand Unified Theories, Revised", Chapter 16, http://wwwpdg.lbl.gov/2016/reviews/rpp2016-revguts.pdf. Retrieved March 2, 2019.

[39] Haber, Howie. (2015), "Supersymmetry, Part I (Theory)",http://pdg.lbl.gov/2015/reviews/rpp2015rev-susy-1-theory.pdf. Retrieved 8 March 2019.

[40] "Theory of everything", https://en.wikipedia.org/wiki/Theory of everything. Retrieved March 2, 2019.

[41] Rovelli, C. (2001). "Notes for a brief history of quantum gravity", https://arxiv.org/pdf/grqc/0006061v3.

[42] Maldacena, Juan (2005). "The Illusion of Gravity". Scientific American. 293 (5): 56-63.

[43] Weinberg, S., Israel, W. (Ed.). (1979). "Ultraviolet divergences in quantum theories of gravitation". United Kingdom: University Press.

[44] Assaf Shomer (2007). "A pedagogical explanation for the non-renormalizability of gravity", https://arxiv.org/pdf/0709.3555v2.

[45] Anselmi, Damiano (2017). "On the quantum field theory of the gravitational interactions", Journal of High Energy Physics, Vol 2017, 6, pp. 86.

[46] Richard P. Feynman, Albert R. Hibbs, Daniel F. Styer, (2010). "Quantum Mechanics and Path Integrals". Mineola, NY: Dover Publications.

[47] Malham, Simon. (2015). "An introduction to Lagrangian and Hamiltonian mechanics". 10.13140/RG.2.1.2914.8003.

[48] Hans Stephani (2004). "Relativity 3ed: An Introduction to Special and General Relativity 3rd Edition", Cambridge University Press; 3rd. edition.

[49] Leonard Susskind, (2017). "Dear Qubitzers, GR=QM", https://arxiv.org/pdf/1708.03040.

[50] Susskind, Leonard (2016). "Copenhagen vs Everett, Teleportation, and ER=EPR", Fortschritte der Physik, 64 (6-7): 551-564. https://arxiv.org/pdf/1604.02589.

[51] Richard MacKenzie (2000). "Path Integral Methods and Applications", https://arxiv.org/pdf/quant$\mathrm{ph} / 0004090$.

[52] P.A.M. Dirac, (1933). "The Lagrangian in Quantum Mechanics", Physikalische Zeitschrift der Sowjetunion, Band 3, Heft 1.

[53] R.P. Feynman, "Space-Time Approach to NonRelativistic Quantum Mechanics", Reviews of Modern Physics 20, 3671948.

[54] "Chapter 9 - General Relativity: The Field Theory Approach", https://javierrubioblog.files.wordpress.com/2015/12/ chapter9.pdf. Retrieved Feb. 16, 2019. 
[55] Pierre Gosselin, Janos Polonyi, (1998). "Path Integral for Relativistic Equations of Motion", Annals of Physics, Volume 268, Issue 2, 20 September 1998, Pages 207-224.

[56] Ian Redmount, Wai-Mo Suen, Kenneth Young (1999). "Ambiguities in Quantizing a Classical System", https://arxiv.org/pdf/gr-qc/9904042v1.

[57] K S Mallesh, S Chaturvedi, V Balakrishnan, R Simon and N Mukunda. (2011). "Symmetries and conservation laws in classical and quantum mechanics", Resonance, March 2011, Volume 16, Issue 3, pp 254-273.

[58] Hagen Kleinert, "Functional-Integral Representation of Quantum Field Theory", http://users.physik.fuberlin.de/-kleinert/b6/psfiles/Chapter-13-functint.pdf . Retrieved dec 12, 2019.

[59] Karen Crowther and Niels Linnemann, (2017), "Renormalizability, fundamentality and a final theory: The role of UV-completion in the search for quantum https://arxiv.org/pdf/1705.06777v2. gravity",

[60] Marc H. Goroff and Augusto Sagnotti, (1986), "The Ultraviolet Behavior of Einstein Gravity", Nuclear Physics B266 (1986) 709-736.

[61] Cumrun Vafa, (2005), "The String Landscape and the Swampland", https://arxiv.org/pdf/hep-th/0509212v2

[62] The Reference Frame, (2017), "Competent formal theorists know they can't rely on the existence of the Lagrangian", https://motls.blogspot.com/2017/11/competentformal-theorists-know-they.html. Retrieved December 18, 2019.

[63] Yuji Tachikawa, "What is Quantum Field Theory?", Kavli IPMU 10th anniversary symposium $16-18$ October 2017.

[64] "Principle of Least Action", http://www.damtp.cam.ac.uk/user/db275/LeastActio n.pdf. Retrieved March 2, 2019.

[65] Jaffe, Arthur, (2000). "Constructive Quantum Field Theory". Review article from Mathematical Physics.

[66] Baez, John C., Segal, I.E. and Zhou, Z., "Introduction to Algebraic and Constructive Quantum Field Theory", Princeton U. Press, 1992

[67] Sourav Chatterjee, (2018), "Yang-Mills for probabilists", https://arxiv.org/pdf/1803.01950v1

[68] Gerald V. Dunne, Mithat Unsal, (2016). "New Methods in QFT and QCD: From Large-N Orbifold Equivalence to Bions and Resurgence", https://arxiv.org/pdf/1601.03414.

[69] James Glimm and Arthur Jaffe (2012). "Quantum physics: a functional integral point of view", Springer
[70] Stephen J. Summers, (2016). "A Perspective on Constructive Quantum Field Theory", https://arxiv.org/pdf/1203.3991v2

[71] P. H. Eberhard and R. R. Ross (1989). "Quantum Field Theory Cannot Provide Faster-Than-Light Communication", Foundations of Physics Letters, Vol. 2, No. 2, 1989

[72] Dick R. (2012), "Klein-Gordon and Dirac Fields. In: Advanced Quantum Mechanics". Graduate Texts in Physics. Springer, New York, NY

[73] Scott Watson, (2001). "Relativistic Path Integrals and the Klein-Gordon Equation", http://www.het.brown.edu/people/watson/papers/rel path_int.ps. Retrieved Feb. 23, 2019.

[74] Ian H. Redmount, Wai-Mo Suen (1992). "Path integration in relativistic quantum mechanics", https://arxiv.org/pdf/grqc/9210019v1.

[75] Guangqing Bi, Yuekai Bi (2010). "Stationary Solutions of the Klein-Gordon Equation in a Potential Field", https://arxiv.org/pdf/1008.4224v4.

[76] "The Klein-Gordon field and its variational principle",

http://www.physics.usu.edu/torre/Classical_Field T heory/Lectures/ 02 KG.pdf. Retrieved Feb. 24, 2019.

[77] Hagen Kleinert, (2004). "Path Integrals in Quantum Mechanics, Statistics, Polymer Physics, and Financial Markets", World Scientific, ISBN 978-981-4365-260 .

[78] Frank Antonsen and Karsten Bormann, (1996). "Propagators in Curved Space", https://arxiv.org/pdf/hep-th/9608141v1

[79] T. S. Bunch and Leonard Parker, (1979). "Feynman propagator in curved spacetime: A momentum-space representation", Physical Review volume 20, Number 10, 15 November 1979

[80] Mayeul Arminjon, (2015). "On the Hamiltonian and energy operators in a curved spacetime, especially for a Dirac particle", J. Phys.: Conf. Ser. 626012030

[81] "Distribution (mathematics)", Wikipedia, https://en.wikipedia.org/wiki/Distribution (mathema tics). Retrieved on Feb 23, 2019.

[82] Sergio Albeverio and Sonia Mazzucchi (2011), "Path Integral: mathematical aspects", Scholarpedia, 6(1):8832,

http://www.scholarpedia.org/article/Path integral: mathematical aspects. Retrieved on Feb 23, 2019.

[83] Carlo Rovelli and Francesca Vidotto, (2014), "Covariant Loop Quantum Gravity: An elementary introduction to Quantum Gravity and Spinfoam Theory", Cambridge University Press.

[84] Jean Zinn-Justin (2009), "Path Integral", Scholarpedia, 4(2):8674. 
[85] Michael Taylor. "Functional Analysis course", http://mtaylor.web.unc.edu/notes/functionalanalysis-course/. Retrieved on Feb. 23, 2019.

[86] Maldacena, Juan and Susskind, Leonard (2013). "Cool horizons for entangled black holes", Fortsch. Phys. $\quad 61 \quad$ (9): $\quad 781-811$. https://arxiv.org/pdf/1306.0533.

[87] Ning Bao and Jason Pollack and Grant N. Remmen, (2015), "Wormhole and entanglement (non)detection in the ER=EPR correspondence", https://arxiv.org/pdf/1509.05426.

[88] Salwa Alsaleh, Lina Alasfar, (2016), "ER= EPR and Non-Perturbative Action Integrals for Quantum Gravity", https://arxiv.org/pdf/1611.02573.

[89] Einstein, A. and Rosen, N. (1935). "The particle problem in the general theory of relativity". Physical Review, 48(1):73.

[90] Eduardo Guendelman, Emil Nissimov, Svetlana Pacheva, Michail Stoilov, (2016),"Einstein-Rosen "Bridge" Revisited and Lightlike Thin-Shell Wormholes", https://arxiv.org/pdf/1611.04336v2

[91] Ning Bao, Aidan Chatwin-Davies, Jason Pollack, Grant N. Remmen, (2018), "Traversable Wormholes as Quantum Channels: Exploring CFT Entanglement Structure and Channel Capacity in Holography", https://arxiv.org/pdf/1808.05963v2.

[92] Mathieu Baillif, Alexandre Gabard (2008), "Manifolds: Hausdorffness versus homogeneity", Proceedings of the American Mathematical Society, 2008, vol. 136, no. 3, p. 1105-1111.

[93] Mark Sharlow, (2007). "The quantum mechanical Path Integral: Toward a realistic interpretation", https://core.ac.uk/download/pdf/11921794.pdf. Retrieved on March 3, 2019

[94] Georges Obied, Hirosi Ooguri, Lev Spodyneiko, Cumrun Vafa, (2018), "De Sitter Space and the Swampland", https://arxiv.org/pdf/1806.08362v3.

[95] Laszlo E. Szabo, (2007), "The Einstein-PodolskyRosen Argument and the Bell Inequalities", https://arxiv.org/pdf/0712.1318v1.

[96] Fiorenzo Bastianelli and Peter van Nieuwenhuizen, (2006). "Path Integrals and Anomalies in Curved Space", Cambridge University Press.

[97] J.A. Shi ett, (2015), "Standard Model Lagrangian (including neutrino mass terms)", http://einsteinschrodinger.com/Standard_Model.pdf, Retrieved December20, 2019.

[98] Y. Aharonov, H. Pendleton, A. Peterson, (1970), "Deterministic Quantum Interference Experiments", Int. J. Th. Phys. 3, 443.

[99] Steven Weinstein, (2008). "Nonlocality without nonlocality", https://arxiv.org/pdf/0812.0349v2.
[100] Mojtaba Ghadimi, Michael J. W. Hall and Howard M. Wiseman, (2018), "Nonlocality in Bell's Theorem, in Bohm's Theory, and in Many InteractingWorlds Theorising", https://arxiv.org/pdf/1807.01568v2.

[101] D. Bohm, (1952), "A Suggested Interpretation of the Quantum Theory in Terms of 'Hidden Variables' I", Phys. Rev. 85 (1952) 166.

[102] D. Bohm, (1952), "A Suggested Interpretation of the Quantum Theory in Terms of 'Hidden Variables' II", Phys. Rev. 85 (1952) 180.

[103] Castro Carlos Perelman, (2018), "Bohm's Potential, Classical/Quantum Duality and Repulsive Gravity", 10.13140/RG.2.2.20005.76006.

[104] HUGH EVERETT (1973), "The Many- Worlds Interpretation of Quantum Mechanics", Princeton University Press

[105] Frank J. Tipler, (2014). "Quantum nonlocality does not exist". Proceedings of the National Academy of Sciences, 2014

[106] Fine, Arthur, "The Einstein-Podolsky-Rosen Argument in Quantum Theory", The Stanford Encyclopedia of Philosophy (Winter 2017 Edition), Edward N. Zalta (ed.), URL = https://plato.stanford.edu/archives/win2017/entries/q t-epr/. Retrieved on March 4. 2019.

[107] Bohr, N., (1935), "Can quantum-mechanical description of physical reality be considered complete?", Physical Review, 48: 696-702.

[108] Sukanya Sinha and Rafael D. Sorkin, (1991), "A Sum-Over-Histories Account of an EPR(B) Experiment", Found. Phys. Letter, 4:303.

[109] C.H. Bennett, G. Brassard, C. Crepeau, R. Jozsa, A. Peres, and W. Wootters, (1993). "Teleporting an Unknown Quantum State via Dual Classical and EPR Channels", Phys. Rev. Lett. vol. 70, pp 1895-1899, 1993.

[110] D. Bouwmeester et al., (1997), "Experimental quantum teleportation", Nature 390, 575-9 (1997)

[111] Fiorenzo Bastianelli, Olindo Corradini, (2017). "On the simplified Path Integral on spheres", https://arxiv.org/pdf/1708.03557v2.

[112] Reartes, Walter. (2004). "Path Integral Quantization of the Sphere", 10.1007/978-1-4419-9058-7_14.

[113] Christian Grosche and Frank Steiner (1995). "How to solve Path Integrals in quantum mechanics", Journal of Mathematical Physics 36, 2354 (1995).

[114] J. A. Wheeler and R. P. Feynman, (1945), "Interaction with the absorber as the mechanism of radiation", Rev. Mod. Phys. 17, 157 (1945). 
[115] Dong Yang, (2006), "A simple proof of monogamy of entanglement", $\mathrm{ph} / 0604168 \mathrm{v} 2$. https://arxiv.org/pdf/quant-

[116] IBM Research, "Quantum Teleportation", https://researcher.watson.ibm.com/researcher/view group.php?id=2862. Retrieved March 7, 2019.

[117] D. N. Matsukevich, P. Maunz, D. L. Moehring, S. Olmschenk, and C. Monroe, (2008). "Bell inequality violation with two remote atomic qubits", https://arxiv.org/pdf/0801.2184v1.

[118] S. Olmschenk, D. N. Matsukevich, P. Maunz, D. Hayes, L.-M. Duan, C. Monroe1, (2009). "Quantum Teleportation Between Distant Matter Qubits", https://arxiv.org/pdf/0907.5240v1.

[119] Elise Crull, (2018), "You thought quantum mechanics was weird: check out entangled time", https://aeon.co/ideas/you-thought-quantummechanics-was-weird-check-out-entangled-time. Retrieved on March 10, 2019.

[120] Christoph Simon, (2002), "Natural entanglement in Bose-Einstein condensates", Phys. Rev. A 66, 052323, Published 25 November 2002.

[121] D. Klauber, (2010), "Derivation of the Feynman Propagator", Student Guide to Quantum Field Theory, Chapter 3, Sandtrove Press.

[122] Jan Louis, "Quantum Field Theory I", http://www.desy.de/jlouis/Vorlesungen/QFTI10/QFTI.pdf. Retrieved Jan 20,2019

[123] L. S. Schulman (2005), "Techniques and Applications of Path Integration", Dover Publications (December 27, 2005).

[124] "Propagator", Wikipedia, https://en.wikipedia.org/wiki/Propagator. Retrieved on February 20, 2019.

[125] Michael E. Peskin and Daniel V. Schroeder. "An Introduction To Quantum Field Theory, Student Economy Edition (Frontiers in Physics)", CRC Press; 1 edition (November 3, 2015).

[126] J.D. Franson, (2008). "Generation of Entanglement Outside of the Light Cone", Journal of Modern Optics $55,2117-2140$.

[127] David Tong, "Quantum Field Theory", http://www.damtp.cam.ac.uk/user/tong/qft.html. Retrieved on February 24, 2019.

[128] Matthias Sonnleitner, Nils Trautmann, and Stephen M. Barnett, "Will a Decaying Atom Feel a Friction Force?", Phys. Rev. Lett. 118, 053601 - Published 3 February 2017.

[129] M. Bordag, U. Mohideen, V.M. Mostepanenko, "New developments in the Casimir effect", Phys. Rep. 353, $1-205,2001$.
[130] Mark D. Roberts, (2001), "Vacuum Energy", https://arxiv.org/pdf/hep-th/0012062v3.

[131] Matthew D. Schwartz (2014), "Quantum Field Theory and the Standard Model", Cambridge University Press, 2014.

[132] Reeh, H., Schlieder, S. (1961). "Bemerkungen zur Unitareiquivalenz von Lorentz invarianten Feldern". Nuovo Cimento, 22, 1051.

[133] Hegerfeldt, G.C. (1998), "Causality, particle localization and positivity of the energy", In Bohm, A., Doebner, H.-D., Kielanowski, P. (Eds.). "Irreversibility and causality: Semigroups and rigged Hilbert spaces", (pp. 238-245). Springer, New York.

[134] Hegerfeldt, G.C. (1998). "Instantaneous spreading and Einstein causality in quantum theory", Annalen der Physik, 7, 716.

[135] Ugo Moschella, (2005). "The de Sitter and anti-de Sitter Sightseeing Tour", Seminaire Poincare 1 (2005) 1 - 12. http://www.bourbaphy.fr/moschella.pdf. Retrieved on February 17, 2019.

[136] A. Einstein, "Kosmologische Betrachtungen zur allgemeinen Relativittstheorie", Sitzungsber. Preuss . Akad. Wiss., Berlin, 142 (1917).

[137] J. M. Maldacena, "Black Holes and Holography in String Theory". In: B. Duplantier and V. Rivasseau (Eds): "Seminaire Poincare", 61-67. (2004).

[138] J. M. Maldacena, (1998), "The Large N Limit Of Superconformal Field Theories And Supergravity", Adv. Theor. Math. Phys. 2 (1998) 231.

[139] "String theory", Wikipedia, https://en.wikipedia.org/wiki/String theory. Retrieved on March 12, 2019.

[140] Maldacena, Juan (2005). "The Illusion of Gravity". Scientific American., 293 (5): 56-63.

[141] Jan Zaanen, Yan Liu, Ya Sun K.Schalm; 2015, "Holographic Duality in Condensed Matter Physics", Cambridge University Press, Cambridge.

[142] Elli Pomoni, (2010). "AdS/CFT beyond the $\mathrm{N}=4$ SYM paradigm", $\mathrm{PhD}$ thesis, Stony Brook University.

[143] van Raamsdonk, Mark (2010). "Building up spacetime with quantum entanglement", Gen. Rel. Grav. 42 (14): 2323-2329. https://arxiv.org/pdf/1005.3035.

[144] P. N. Kaloyerou, (1995). "Causal Interpretation of the Modified Klein-Gordon Equation", Foundations of Physics. Vol. 25, No. 10. 1995.

[145] A. Einstein (1925). "Quantentheorie des einatomigen idealen Gases". Sitzungsberichte der Preussischen Akademie der Wissenschaften 1: 3 
[146] Bardeen, J.; Cooper, L. N.; Schrieffer, J. R. (April 1957). "Microscopic Theory of Superconductivity". Physical Review. 106 (1): 162-164.

[147] V. L. Ginzburg \& L.D. Landau (1950). "On the theory of superconductivity". Zhurnal Eksperimental noi i Teoreticheskoi Fiziki. / JETP 20: 1064.

[148] Qijin Chen, Jelena Stajic, Shina Tan, K. Levin, (2004), "BCS-BEC crossover: From high temperature superconductors to ultracold superfluids", https://arxiv.org/pdf/cond-mat/0404274v3.

[149] Sachdev S, (2013), "Strange and stringy", Sci Am., 2013 Jan, 308(1):44-51.

[150] Itai Panas, (2012), "Superatom Representation of High-TC Superconductivity", Physica C: Superconductivity Volume 480, October 2012, Pages 137-143.

[151] J. Zaanen and al. (2006), "Towards a complete theory of high T-c", Nature Physics volume 2, pages 138143.

[152] M. Ulmke, V. Janis, D. Vollhardt (1994). "AndersonHubbard Model in $\mathrm{d}=\infty "$ ", https://arxiv.org/pdf/condmat/9411034v1.

[153] Tobias Wenger, (2012), "Holographic Superconductivity Effective Field Theoretic Approach to Layered Superconductors", MS Thesis, Chalmers University of Technology Gothenburg, Sweden.

[154] Rong-Gen Cai, Li Li, Li-Fang Li, Run-Qiu Yang. (2015), "Introduction to holographic superconductor models", https://arxiv.org/pdf/1502.00437v3.

[155] Mohit Randeria and Edward Taylor, (2014). "BCSBEC Crossover and the Unitary Fermi Gas", https://arxiv.org/pdf/1306.5785v3.

[156] Clovis Jacinto de Matos, Martin Tajmar (2006). "Gravitomagnetic London Moment and the Graviton Mass inside a Superconductor", https://arxiv.org/pdf/cond-mat/0602591.

[157] Ryder, L.H.(1996), "Quantum Field Theory", Cambridge University Press, 2nd Edition, 1996

[158] Y. Cao, V. Fatemi, S. Fang, K. Watanabe, T. Taniguchi, E. Kaxiras, and P. Jarillo-Herrero, "Unconventional superconductivity in magic-angle graphene superlattices", Nature 556, 43 (2018).

[159] Maddury Somayazulu, Muhtar Ahart, Ajay K Mishra, Zachary M. Geballe, Maria Baldini, Yue Meng, Viktor V. Struzhkin, Russell J. Hemley. (2018). "Evidence for superconductivity above $260 \mathrm{~K}$ in lanthanum superhydride at megabar pressures", https://arxiv.org/pdf/1808.07695v3.

[160] A. P. Drozdov, V. S. Minkov, S. P. Besedin, P. P. Kong, M. A. Kuzovnikov, D. A. Knyazev, M. I. Eremets (2018). "Superconductivity at $215 \mathrm{~K}$ in lanthanum hydride at high pressures". https://arxiv.org/pdf/1808.07039v1.

[161] Bogdan Opanchuk, Rodney Polkinghorne, Oleksandr Fialko, Joachim Brand, Peter D.Drummond (2013). "Quantum simulations of the early universe", https://arxiv.org/pdf/1305.5314v2.

[162] Jean-Paul Blaizot, Bin Wu, and Li Yan (2014), "Quark production, Bose-Einstein condensates and thermalization of the quark-gluon plasma", Nucl.Phys. A930 (2014) 139-162.

[163] ALICE Collaboration, (2018), "Anisotropic flow in xexe collisions at sqrtsNN $=5.44 \mathrm{TeV}$, https://arxiv.org/pdf/1805.01832v2.

[164] "Quark-gluon plasma", Wikipedia, https://en.wikipedia.org/wiki/Quark\%E2\%80\%93glu on plasma. Retrieved April 6, 2019.

[165] A D Linde, (1984). "The inflationary universe", Rep. Prog. Phys., Vol 47, pp 925-986, 1984.

[166] A. Guth, (2018), "The New Inflationary Universe", MIT Course, Physics 8.286: The Early Universe.

[167] "Chronology of the universe", https://en.wikipedia.org/wiki/Chronology of the un iverse. Retrieved on April 6. 2019.

[168] "Quantum Computing", Wikipedia, https://en.wikipedia.org/wiki/Quantum computing. Retrieved April 7, 2019

[169] Domenico Giulini, (2012), "Equivalence Principle, Quantum Mechanics, and Atom-Interferometric Tests", Quantum Field Theory and Gravity pp 345370. Springer, Basel.

[170] E. Kajari, N.L. Harshman, E.M. Rasel, S. Stenholm, G. Süssmann, W.P. Schleich, (2010), "Inertial and gravitational mass in quantum mechanics", https://arxiv.org/pdf/1006.1988v2.

[171] Carroll, S., "Spacetime and Geometry. An Introduction to General Relativity", (Pearson Education Limited, 2014).

[172] Rashmi Shivni, (2016). "The deconstructed Standard Model equation", https://www.symmetrymagazine.org/article/thedeconstructed-standard-model-equation. Retrieved on February 24, 2019.

[173] Gernot Eichmann, (2014), "Poincaré group", http:/cftp.ist.utl.pt/gernot.eichmann/2014-hadronphysics/hadron-app-2.pdf. Retrieved on April 15, 2019.

[174] Sougato Bose, (2018). "A Spin Entanglement Witness for Quantum Gravity", https://arxiv.org/pdf/1707.06050v1.

[175] C. Marletto and V. Vedral, (2018). "Gravitationallyinduced entanglement between two massive particles 
is sufficient evidence of quantum effects in gravity". https://arxiv.org/pdf/1707.06036v2.

[176] C. Anastopoulos and B. L. Hu. (2018). "Comment on "A Spin Entanglement Witness for Quantum Gravity" and on "Gravitationally Induced Entanglement between Two Massive Particles is Sufficient Evidence of Quantum Effects in Gravity" ". https://arxiv.org/pdf/1804.11315v2.

[177] Moore, Thomas A., (2013), "A General Relativity Workbook", University Science Books.

[178] Mordehai Milgrom, (2014), "The MOND paradigm of modified dynamics", Scholarpedia, 9(6):31410.

[179] Tower Wang, (2012), "Modified entropic gravity revisited", https://arxiv.org/pdf/1211.5722v1.

[180] Zhi-Wei Wang and Samuel L. Braunstein., (2018), "Surfaces away from horizons are not thermodynamic", Nature Communications 9, Article number: 2977 (2018).

[181] Pardo, Kris (2017-06-02). "Testing Emergent Gravity with Isolated Dwarf Galaxies" (Report). https://arxiv.org/pdf/1706.00785.

[182] Wikipedia, "Divergence theorem", https://en.wikipedia.org/wiki/Divergence theorem. Retrieved December 27, 2019.

[183] Wikipedia, "Gauss's law for gravity", https://en.wikipedia.org/wiki/Gauss $\% 27$ s law for_g ravity. Retrieved December 27, 2019.

[184] Charles W. Misner and John A. Wheelers, (1957), "Classical Physics as Geometry. Gravitation, Electromagnetism, Unquantized Charge, and Mass as Properties of Curved Empty Space", Annals Of Physics, 2, 525-603.

[185] Luca Bombelli, Rafael Sorkin, Joohan Lee, (1986), "Quantum source of entropy for black holes", Physical Review D, 1986.

[186] Saurya Das, S. Shankaranarayanan, (2007), "Entanglement as a source of black hole entropy", https://arxiv.org/pdf/gr-qc/0610022v2.

[187] Christopher Eling, Raf Guedens, Ted Jacobson, (2006), "Non-equilibrium Thermodynamics of Spacetime", https://arxiv.org/pdf/gr-qc/0602001v1.

[188] Kristan Jensen, Andreas Karch (2013), "The holographic dual of an EPR pair has a wormhole", https://arxiv.org/pdf/1307.1132.

[189] Padmanabhan, T., (2008), "Gravity: The inside story". Gen.Rel.Grav. 40 (2008) 2031-2036, Int.J.Mod.Phys. D17 (2009) 2585-2591.

[190] ChunJun Cao, Sean M. Carroll, Spyridon Michalakis, (2016). "Space from Hilbert Space: Recovering Geometry from Bulk Entanglement", https://arxiv.org/pdf/1606.08444v3.
[191] J. Makela, (2009), "A Simple Quantum-Mechanical Model of Spacetime I: Microscopic Properties of Spacetime", https://arxiv.org/pdf/0805.3952v3.

[192] J. Makela, (2009) "A Simple Quantum-Mechanical Model of Spacetime II: Thermodynamics of Spacetime", https://arxiv.org/pdf/ 0805.3955v3.

[193] J. Makela (2008), "Partition Function of Spacetime", https://arxiv.org/pdf/0810.4910v1.

[194] Jarmo Makela (2010), "Notes Concerning "On the Origin of Gravity and the Laws of Newton" by E. Verlinde (https://arxiv.org/pdf/1001.0785)", https://arxiv.org/pdf/1001.3808v3.

[195] Viqar Husain, R. B. Mann, (2008), "Thermodynamics and phases in quantum gravity", https://arxiv.org/pdf/0812.0399v2.

[196] Magdalena Zych, Fabio Costa, Igor Pikovski and Caslav Brukner, (2019), "Bell's theorem for temporal order", Nature Communications volume 10, Article number: 3772.

[197] Lee Smolin, (2015). "Quantum mechanics and the principle of maximal variety". https://arxiv.org/pdf/1506.02938v1.

[198] Lee Smolin, (2019). "A casual theory of views". https://arxiv.org/pdf/1712.04799v2.

[199] Lee Smolin, (2011). "A real ensemble interpretation of quantum mechanics", https://arxiv.org/pdf/1104.2822v1.

[200] xiao Dong, Ling Zhou, (2018). "Spacetime as the optimal generative network of quantum states: a roadmap to $\mathrm{QM}=\mathrm{GR}$ ?", https://arxiv.org/pdf/1804.07908v1.

[201] G. Evenbly and G. Vidal. (2011). "Tensor network states and geometry". Journal of Statistical Physics, 145(4):891-918, 2011.

[202] Roger Penrose, (1972), "On the nature of quantum geometry", in "Magic Without Magic", ed. J. Klauder, Freeman, San Francisco.

[203] Fotini Markopoulou, Lee Smolin (2004). "Quantum Theory from Quantum Gravity". https://arxiv.org/pdf/gr-qc/0311059v2.

[204] E. Nelson, "Derivation of the Schrodinger equation from Newtonian mechanics", Phy. Rev., 150, 1079 (1969); "Quantum Fluctuations", Princeton Series in Physics, Princeton University Press (1985).

[205] Hermann Nicolai and Kasper Peeters, (2006). "Loop and Spin Foam Quantum Gravity: A Brief Guide for Beginners", https://arxiv.org/pdf/hep-th/0601129v2.

[206] Hou Y. Yau, (2007 \& 2016), "Quantum Theory from a Space-Time Wave", https://arxiv.org/pdf/0706.0190 $\underline{\mathrm{v} 2}$ and $\underline{\mathrm{v} 4}$. 
[207] Ekert A., Jozsa R., Penrose R., and Penrose Roger (1999). "Quantum computation, entanglement and state reduction". 356 Philosophical Transactions of the Royal Society of London. Series A: Mathematical, Physical and Engineering Sciences.

[208] Tejinder P. Singh, (2018). "Space and Time as a Consequence of GRW Quantum Jumps". https://arxiv.org/pdf/1806.01297v4.

[209] Tejinder P. Singh, (2018). "Space-time from Collapse of the Wave-function", https://arxiv.org/pdf/1809.03441v2.

[210] Wayne C. Myrvold, (2014). "What is a Wavefunction?", $\quad$ http://philsciarchive.pitt.edu/10750/. Retrieved on April 22, 2019.

[211] G N Ord, (1983), "Fractal space-time: a geometric analogue of relativistic quantum mechanics", J. Phys. A: Math. Gen. 16 (1983) 1869-1884.

[212] Laurent Nottale, (2010), "Scale Relativity and Fractal Space-Time: Theory and Applications", Found of Sciences, June 2010, Volume 15, Issue 2, pp 101-152.

[213] Michael R. Douglas, Nikita A. Nekrasov, (2001), "Noncommutative Field Theory", https://arxiv.org/pdf/hep-th/0106048v4.

[214] Louise Dolan, Chiara R. Nappi, (2033), "Strings and Noncommutativity", https://arxiv.org/pdf/hepth/0302122v2.

[215] Florian Girelli, Franz Hinterleitner and Seth A. Major, (2012), "Loop Quantum Gravity Phenomenology: Linking Loops to Observational Physics", "Symmetry, Integrability and Geometry: Methods and Applications" SIGMA 8 (2012), 098, 73 pages.

[216] "Dark Energy", Wikipedia, https://en.wikipedia.org/wiki/Dark energy. Retrieved on February 27, 2019.

[217] Joel Primack, "Historical Introduction to $\Lambda \mathrm{CDM}$ Cosmology", http://physics.ucsc.edu/-joel/PrimackLect1-LCDM History.pdf. Retrieved on May 12, 2019.

[218] Qiaoling Yang, (2012), "Axion BEC: A Model Beyond CDM", PhD Thesis, UNIVERSITY OF FLORIDA.

[219] Svend Erik Rugh, Henrik Zinkernagel, (2000). "The Quantum Vacuum and the Cosmological Constant Problem", https://arxiv.org/pdf/hep-th/0012253v1.

[220] Wm. Robert Johnston, (2008). "Calculations on space-time curvature within the Earth and Sun", http://www.johnstonsarchive.net/relativity/stcurve.p df. Retrieved on May 6, 2019.

[221] J. V. Narlikar, Ganeshkhind, J.-C. Pecker and J.-P. Vigier, (1991), "Some Consequences of a Spatially Varying Cosmological Constant in a Spherically
Symmetric Distribution of Matter", J. Astrophys. Astr. 12, 7-16.

[222] Hongya Liu and Paul S. Wesson, (2001). "Universe Models WITH A Variable Cosmological And A Big Bounce", The Astrophysical Journal, 562 : 16, 2001 November 20

[223] "Dark Matter", Wikipedia, https://en.wikipedia.org/wiki/Dark matter. Retrieved on February 27, 2019.

[224] M. Tajmar, F. Plesescu, K. Marhold, C.J. de Matos. (2006), "Experimental Detection of the Gravitomagnetic London Moment", https://arxiv.org/pdf/gr-qc/0603033v1.

[225] Martin Tajmar, Florin Plesescu, Bernhard Seifert, Klaus Marhold (2006), "Measurement of Gravitomagnetic and Acceleration Fields Around Rotating Superconductors", https://arxiv.org/pdf/grqc/0610015.

[226] M. Tajmar, F. Plesescu, B. Seifert, R. Schnitzer, I. Vasiljevich, (2008), "Search for Frame-DraggingLike Signals Close to Spinning Superconductors", https://arxiv.org/pdf/0707.3806v9.

[227] R.M. Wald, (1984), "General Relativity", The University of Chicago Press.

[228] Wytler Cordeiro dos Santos, (2016), "Introduction to Einstein-Maxwell equations and the Rainich conditions", https://arxiv.org/pdf/1606.08527v1.

[229] I. C. Jardima, R. R. Landim, (2013), "Deviation of Large Scale Gravitoelectromagnetic Field in PostNewtonian https://arxiv.org/pdf/1304.6385v2.

[230] Juan Maldacena, Alexey Milekhin, Fedor Popov, (2018). "Traversable wormholes in four dimensions", https://arxiv.org/pdf/1807.04726v2.

[231] Faizuddin Ahmed, Bidyut Bikash Hazarika and Debojit Sarma (2016), "The anti-de Sitter spacetime as a time machine", Eur. Phys. J. Plus (2016) 131:230.

[232] G. 't Hooft, (1993), "Dimensional Reduction in Quantum Gravity", https://arxiv.org/pdf/grqc/9310026v2.

[233] Ahmed Almheiri, xi Dong, Daniel Harlow, (2014), "Bulk Locality and Quantum Error Correction in AdS/CFT", https://arxiv.org/pdf/1411.7041v3.

[234] Fernando Pastawski, Beni Yoshida, Daniel Harlow, John Preskill, (2015), "Holographic quantum errorcorrecting codes: Toy models for the bulk/boundary correspondence", https://arxiv.org/pdf/1503.06237v2.

[235] Ahmed Almheiri, (2018), "Holographic Quantum Error Correction and the Projected Black Hole Interior", https://arxiv.org/pdf/1810.02055v2. 
[236] Sabine Hossenfelder, (2018), "Lost in Math: How Beauty Leads Physics Astray", Basic Books.

[237] Lee Smolin, (2006), "The Trouble with Physics: The Rise of String Theory, The Fall of a Science, and What Comes Next", Mariner Books.

[238] Peter Woit, (2007), "Not Even Wrong: The Failure of String Theory and the Search for Unity in Phyisical Law", Basic Books

[239] Wikipedia, "Proton decay", https://en.wikipedia.org/wiki/Proton_decay. Retrieved, January 3, 2020.

[240] Ethan Siegel and al., (2020), "How Certain Are We That Protons Don't Decay?", https://www.forbes.com/sites/startswithabang/2020/ 01/03/how-certain-are-we-that-protons-dont-decay. Retrieved, January 3, 2020.

[241] W. Zhu, Zhoushen Huang, Yin-chen He, (2018), "Reconstructing Entanglement Hamiltonian via Entanglement https://arxiv.org/pdf/1806.08060.

[242] Fabio Maria Mele, (2016), "Quantum Metric and Entanglement on Spin Networks", https://arxiv.org/pdf/1703.06415v1.

[243] Johannes Thueringen, (2015), "Discrete quantum geometries and their effective dimension", $\mathrm{Ph} . \mathrm{D}$. Thesis, Humboldt-Universitat zu Berlin

[244] Helge Kragh, (2002), "Quantum Generations: A History of Physics in the Twentieth Century", Princeton University Press; Reprint edition (March 24, 2002).

[245] K. A. Milton, (2015), "Schwinger's Quantum Action Principle: From Dirac's formulation through Feynman's Path Integrals, the Schwinger-Keldysh method, quantum field theory, to source theory", https://arxiv.org/pdf/1503.08091v1.

[246] A. M. Ozorio de Almeida, (2006), "Entanglement in phase space", $\quad$ https://arxiv.org/pdf/quant$\mathrm{ph} / 0612029 \mathrm{v} 1$.

[247] The reference frame, (2012), "Why Feynman's Path Integral doesn't contradict the uncertainty principle", https://motls.blogspot.com/2012/06/why-feynmanspath-integral-doesnt.html. Retrieved Dec 15, 2019.

[248] Lee Smolin, (2006), "The Trouble with Physics", Houghton Mifflin Harcourt.

[249] Lee Smolin, (2005), "The case for background independence", $\quad$ https://arxiv.org/pdf/hepth/0507235v1.

[250] Peter Collas, (1977), "General relativity in two- and three-dimensional space-times", American Journal of Physics, Vol. 45, No.9, September 1977.
[251] S. Carlip, (2004), "Quantum Gravity in 2+1 Dimensions: The Case of a Closed Universe", https://arxiv.org/pdf/gr-qc/0409039v2.

[252] Zhengjun xi, Yongming Li and Heng Fan, (2015), "Quantum coherence and correlations in quantum system", Nature, Scientific Reports volume 5, Article number: 10922 .

[253] Dai, J., Leigh, R. G., and Polchinski, J. (1989). "New connections between string theories", Modern Physics Letters A, 04(21): 2073-2083.

[254] Anze Zaloznik, (2012), "Kaluza-Klein Theory", http://mafija.fmf.uni-

lj.si/seminar/files/2011_2012/KaluzaKlein_theory.pd f. Retrieved Jan 12, 2020.

[255] Edward Witten, (1995), "String Theory Dynamics In Various Dimensions", https://arxiv.org/pdf/hepth/9503124v2.

[256] David Bailint and Alex Love, (1987), "Kaluza-Klein theories", Rep. Prog. Phys. 50 (1987) 1087-1170.

[257] Alain Aspect, (2004), "Bell's Theorem: The Naive View Of An Experimentalist", https://arxiv.org/pdf/quant-ph/0402001v1.

[258] Julia Cramer, Christian Schiitte-Niitgen, (2010), "Experimental Violations of Bell's Inequalities", https:/qudev.phys.ethz.ch/static/content/courses/QSI T10/presentations/QSIT-BellsInequality.pdf. Retrieved on Jan 12, 2020.

[259] W.M. Stuckey, Michael Silberstein, Timothy McDevitt and Ian Kohler, (2019), "Why the Tsirelson Bound? Bub's Question and Fuchs' Desideratum", https://arxiv.org/pdf/1807.09115v7.

[260] Katrin Becker, Melanie Becker and John L. Schwarz, (2007), "String Theory and M-Theory. A Modern Introduction", Cambridge University Press.

[261] Rahul Sawant, Joseph Samuel, Aninda Sinha, Supurna Sinha, Urbasi Sinha, (2014), "Non-classical paths in interference experiments", https://arxiv.org/pdf/1308.2022v2.

[262] R. Wald, (1980), "Quantum gravity and time reversibility", Phys. Rev. D21 (1980), 2742.

[263] Nick E. Mavromatos, (2005), "CPT Violation: Theory and Phenomenology", https://arxiv.org/pdf/hep$\mathrm{ph} / 0504143 \mathrm{v} 1$.

[264] Jaeger, Gregg (2019). "Are virtual particles less real?", Entropy, 21 (2): 141.

[265] Mary Bell, Shan Gao, (2016), "Quantum Nonlocality and Reality. 50 Years of Bell's Theorem", Cambridge University Press.

[266] H. Reeh, S. Schlieder, (1961), "Bemerkungen zur unitaraquivalenz von lorentzinvarianten feldern", Il 
Nuovo Cimento (1955-1965), December 1961, Volume 22, Issue 5, pp 1051-1068.

[267] Joseph J. Bisognano and Cyvind H. Wichmann, (1975), "On The Duality Condition For A Hermitian Scalar Field", Journal of Mathematical Physics, Vol. 16, No. 4, 985 - 1007.

[268] Wikipedia, "Reeh-Schlieder theorem", https://en.wikipedia.org/wiki/ReehSchlieder theorem. Retrieved Nov. 19, 2019.

[269] nLab, "Reeh-Schlieder theorem", https://ncatlab.org/nlab/show/ReehSchlieder+theorem. Retrieved Nov. 19, 2019.

[270] Luciano Combi, Gustavo E. Romeroa, (2017), "Is Teleparallel Gravity really equivalent to General Relativity?", https://arxiv.org/pdf/1708.04569v1.

[271] V. C. De Andrade, L. C. T. Guillen and J. G. Pereira, (2000), "Teleparallel Gravity: An Overview", https://arxiv.org/pdf/gr-qc/0011087v1.

[272] Bo-Sture K. Skagerstam, (1976), "Some remarks concerning the question of localization of elementary particles", International Journal of Theoretical Physics, 15(3):213-230, February 1976.

[273] Hans Halvorson, Rob Clifton, (2001), "No place for particles in relativistic quantum theories?", https://arxiv.org/pdf/quant-ph/0103041v1.

[274] Martin Schottenloher, (2008), "A Mathematical Introduction to Conformal Field Theory (Lecture Notes in Physics)", Springer; 2nd edition (November 17, 2008).

[275] Makoto Natsuume, (2015), "AdS/CFT Duality User Guide (Lecture Notes in Physics)", Springer; 2015 edition (April 2, 2015).

[276] Horatiu Nastase, (2007), "Introduction to AdS-CFT", https://arxiv.org/pdf/0712.0689v2

[277] John Campbell,(2018),"The Black Book of Quantum Chromodynamics: A Primer for the LHC Era", Oxford University Press.

[278] Walter Greiner, Stefan Schramm, Eckart Stein, (2007), "Quantum Chromodynamics", Springer.

[279] Jared Kaplan, (2016), "QFT Lectures Notes", Department of Physics and Astronomy, Johns Hopkins University.

[280] Frank Wilczek, (2012), "Origins of Mass", https://arxiv.org/pdf/1206.7114v2.

[281] Diego Bettoni, (2012), "Masses and the Higgs Mechanism", $\quad$ http://www.fe.infn.it/bettoni/particelle/Strong/HiggsMechanism.pdf. Retrieved on January 20, 2020.
[282] Magdalena Zych, Caslav Brukner, (2015), "Quantum formulation of the Einstein Equivalence Principle", https://arxiv.org/pdf/1502.00971v1.

[283] A. Einstein, " Uber das Relativitatsprinzip und die aus demselben gezogenen Folgerungen". Jahrb. f. Rad. und Elekt. 4, 411 (1907).

[284] Wikipedia, "Equivalence principle", https://en.wikipedia.org/wiki/Equivalence principle. Retrieved on January 20, 2020.

[285] L. Prochaska, x. Li, D. C. MacFarland, A. M. Andrews, M. Bonta, E. F. Bianco, S. Yazdi, W. Schrenk, H. Detz, A. Limbeck, Q. Si, E. Ringe, G. Strasser, J. Kono, S. Paschen, (2018), "Singular charge fluctuations at a magnetic quantum critical point", https://arxiv.org/pdf/1808.02296v1.

[286] P. W. Anderson, (1963), "Plasmons, Gauge Invariance, and Mass", Phys. Rev. 130, 439.

[287] Yao Wang, Yi-Jun Chang, Jun Gao, Yong-Heng Lu, Zhi-Qiang Jiao, Fang-Wei Ye, xian-Min Jin, (2019), "Observation of Magic Angle and Wall State in Twisted Bilayer Photonic Graphene", https://arxiv.org/pdf/1911.09174v1.

[288] F. W. Hehl, (1973), "Spin and torsion in general relativity: I. Foundations", General Relativity and Gravitation. July 1973, Volume 4, Issue 4, pp 333 349.

[289] Friedrich W. Hehl, Paul von der Heyde, and G. David Kerlick, (1976), "General relativity with spin and torsion: Foundations and prospects", Reviews of Modern Physics, Vol. 48, No. 3, July 1976.

[290] Stefan Lippoldt, 2016, "Fermions in curved spacetimes", Thesis, Friedrich-Schiller-Universitat Jena.

[291] Stefano Lucat, Tomislav Prokopec, (2015), "Cosmological singularities and bounce in CartanEinstein theory", https://arxiv.org/pdf/1512.06074v1.

[292] Yi-Fu Cai, Salvatore Capozziello, Mariafelicia De Laurentis, Emmanuel N. Saridakis, (2016), "f(T) teleparallel gravity and cosmology", https://arxiv.org/pdf/1511.07586v2.

[293] Eckehard W. Mielke, (2013), "Is Einstein-Cartan Theory Coupled to Light Fermions Asymptotically Safe?", Journal of Gravity, Volume 2013, Article ID 812962.

[294] M. Gockeler, T. Schiicker, (1987), "Differential Geometry, Gauge Theories and Gravity", Crambridge University Press.

[295] Andrzej Trautman, (2006), "Einstein-Cartan Theory", https://arxiv.org/pdf/gr-qc/0606062v1

[296] Nikodem Poplawski, (2012), "Nonsingular, bigbounce cosmology from spinor-torsion coupling", https://arxiv.org/pdf/1111.4595v2. 
[297] Jordan L. Cubero, Nikodem J. Poplawski, (2019), "Analysis of big bounce in Einstein-Cartan cosmology", https://arxiv.org/pdf/1906.11824v1.

[298] Ilya L. Shapiro, Poliane M. Teixeira, (2014), "Quantum Einstein-Cartan theory with the Holst term", https://arxiv.org/pdf/1402.4854v2

[299] Marc Geiller and Karim Noui, (2013), "A note on the Holst action, the time gauge, and the Barbero-Immirzi parameter", https://arxiv.org/pdf/1212.5064v2

[300] Danilo Jimenez Rezende, Alejandro Perez, (2009), "4d Lorentzian Holst action with topological terms", https://arxiv.org/pdf/0902.3416v1.

[301] Ilya L. Shapiro, (1998), "Torsion: theory and possible observables", $\quad$ https://arxiv.org/pdf/hepth/9811072v1.

[302] Bahram Mashhoon, (2001), "Gravitoelectromagnetism: A Brief Review", https://arxiv.org/pdf/gr-qc/0311030v2.

[303] Ignazio Ciufolini and John Archibald Wheeler, (1995), "Gravitation and Inertia", Princeton University Press.

[304] david G. Boulware and S. Deser, (1975), "Classical General Relativity Derived from Quantum Gravity", Annals Of Physics, 89, 193-240 (1975).

[305] V.M. Red'kov, N.G. Tokarevskaya, V.V. Kisel, (2011), "Graviton in a Curved Space-Time Background and Gauge https://arxiv.org/pdf/1109.1382v1. Symmetry",

[306] Per Kraus and E. T. Tomboulis, (2002), "Photons and Gravitons as Goldstone Bosons, and the Cosmological Constant", https://arxiv.org/pdf/hepth/0203221v1.

[307] Claudia de Rham, Gregory Gabadadze, Andrew J. Tolley, (2010), "Resummation of Massive Gravity", https://arxiv.org/pdf/1011.1232v2.

[308] Cianfrani Francesco, Giovanni Montani, and Lecian Orchidea Maria, (2014), "Canonical Quantum Gravity: Fundamentals and Recent Developments", World Scientific Publishing Company, Incorporated.

[309] xi Dong, Eva Silverstein, Gonzalo Torroba, (2018), "De Sitter Holography and Entanglement Entropy", https://arxiv.org/pdf/1804.08623v2.

[310] Brian Clegg, (2019), "Dark Matter and Dark Energy: The Hidden $95 \%$ of the Universe", Icon Books Ltd.

[311] J. D. Bekenstein, (1973), "Black Holes and Entropy", Phys. Rev. D7 (1973) 2333.

[312] Jacob D. Bekenstein, (1974), "Generalized second law of thermodynamics in black-hole physics", Physical Review D, Volume 9, Number 12, 15 June 1974.
[313] S. W. Hawking, (1975), "Particle Creation by Black Holes", Commun. math. Phys. 43, 199-220 (1975).

[314] Piotr T. Chru \pm ciel, Erwann Delay, Gregory J. Galloway, Ralph Howard, (2000), "Regularity of Horizons and The Area Theorem", https://arxiv.org/pdf/gr-qc/0001003v2.

[315] G. W. Gibbons' and S. W. Hawking, (1977), "Cosmological event horizons, thermodynamics, and particle creation", Physical Review D, Volume 15, Number 10

[316] Ted Jacobson, (2000), "On the Nature of Black Hole Entropy", https://arxiv.org/pdf/gr-qc/9908031v2.

[317] Ryu Shinsei, Takayanagi Tadashi, (2006), "Aspects of Holographic Entanglement Entropy". Journal of High Energy Physics., 2006 (8).

[318] L. Susskind, (1994), "The World as a Hologram", https://arxiv.org/pdf/hep-th/9409089v2.

[319] Paul Dirac, (1931), "Quantised Singularities in the Electromagnetic Field". Proc. Roy. Soc., (London) A 133, 60 (1931).

[320] Yang C.N. (1996), "Magnetic Monopoles, Fiber Bundles, and Gauge Fields". In: Newman H.B., Ypsilantis T. (eds) "History of Original Ideas and Basic Discoveries in Particle Physics". NATO ASI Series (Series B: Physics), vol 352. Springer, Boston, MA.

[321] "Magnetic monopole", Wikipedia, https://en.wikipedia.org/wiki/Magnetic_monopole. Retrieved March 10, 2020.

[322] Bryce DeWitt, (2013), "The Global Approach to Quantum Field Theory", Oxford U. Press, New York, 2003. Vols. 1 and 2.

[323] Richard Feynman, (2002), "Feynman Lectures On Gravitation", Westview Press; 1 edition (June 20, 2002).

[324] I. I. Bigi and A. I. Sanda, (2009), "CP Violation", Cambridge University Press.

[325] Alberto Rojo, Anthony Bloch (2018), "The Principle of Least Action: History and Physics", Cambridge University Press.

[326] Francisco S. N. Lobo, Gonzalo J. Olmo, D. RubieraGarcia, (2014), "Microscopic wormholes and the geometry of entanglement", https://arxiv.org/pdf/1402.5099v2.

[327] Fabrizio Tamburini, Ignazio Licata, (2019), "General Relativistic Wormhole Connections from PlanckScales and the ER = EPR Conjecture", https://arxiv.org/pdf/1912.12424v1.

[328] Jiunn-Wei Chen, Sichun Sun, Yun-Long Zhang, (2016), "Bell Inequality in the Holographic EPR Pair", https://arxiv.org/pdf/1612.09513v3 
[329] Hilary Greaves and Teruji Thomas, (2014), "On the CPT theorem", Studies in History and Philosophy of Modern Physics 45, (2014), 46-65.

[330] Streater, R. and Wightman, A., (1964), "PCT, spin and statistics, and all that". NewYork: W.A.Benjamin.

[331] Stefan Hollands, (2004), "PCT Theorem for the Operator Product Expansion in Curved Spacetime", Commun. Math. Phys. 244, 209-244 (2004).

[332] Wheeler, John Archibald (1963). "Geometrodynamics". New York: Academic Press.

[333] Edward Witten, (1982), "Instability of the KaluzaKlein vacuum", Nuclear Physics B, Volume 195, Issue 3, 22 February 1982, Pages 481-492.

[334] Malcolm Fairbairn, Robert Hogan, (2014), "Electroweak Vacuum Stability in light of BICEP2", https://arxiv.org/pdf/1403.6786v3.

[335] John G. Cramer, (2016),"The Quantum Handshake: Entanglement, Nonlocality and Transactions", Springer.

[336] Graham P. Collins, (2017), "The Many Interpretations of Quantum Mechanics", Scientific American, Nov 2017.

[337] D.Z. Albert, Y. Aharonov, S. D'Amato. Phys. Rev. Lett. 54, 5 (1985), "Curious New Statistical Prediction of Quantum Mechanics", Phys. Rev. Lett. $54,5$.

[338] Y. Aharonov, P.G. Bergmann, J.L. Lebowitz, (1964),"Time Symmetry in the Quantum Process of Measurement", Phys. Rev. 134, B1410 (1964).

[339] Padmanabhan, T., (1994), "Path Integral for the relativistic particle and harmonic oscillators", Found Phys 24, 1543-1562 (1994).

[340] G Rengaraj, U Prathwiraj, Surya Narayan Sahoo, R Somashekhar and Urbasi Sinha, (2018), "Measuring the deviation from the superposition principle in interference experiments", New J. Phys. 20 (2018).

[341] John Preskill, (1984), "Magnetic Monopoles", Ann. Rev. Nucl. Part. Sci., 1984. 34:461-530.

[342] Ivan Agullo, Adrian del Rio, and Jose Navarro-Salas, (2017), "Electromagnetic Duality Anomaly in Curved Spacetimes", Phys. Rev. Lett. 118, 111301.

[343] J. L. Hewett et al. (2014), "Planning the Future of U.S. Particle Physics (Snowmass 2013): Chapter 2: Intensity Frontier", https://arxiv.org/pdf/1401.6077v1.

[344] Rune Fisker, (2016), "Grand Unification Dream Kept at Bay", https://www.quantamagazine.org/no-protondecay-means-grand-unification-must-wait20161215/. Retrieved on Nov 8, 2019.
[345] A. A. Saharian, "Quantum Field Theory In Curved Spacetime", http://training.hepi.tsu.ge/rtn/activities/sources/Lect QFTrev.pdf. Retrieved March 5, 2020.

[346] Dag-Morten Sj0str0m, (2013), "Bosons and Fermions in Curved Spacetime", Physics Thesis, Norwegian University of Science and Technology, May 2013.

[347] Wikipedia, "Bimetric gravity", https://en.wikipedia.org/wiki/Bimetric gravity. Retrieved on January 23, 2020.

[348] Rosen, Nathan (1973), "A bi-metric Theory of Gravitation", Gen. Rel. Grav., 4 (6): 435-447.

[349] P. C. Aichelburg and R. U. Sexl, (1971), "On the Gravitational Field of a Massless Particle", General Relativity and Gravitation, Vol. 2, No. 4 (1971), pp. 303-312.

[350] N. A. Voronov and I. Yu. Kobzarev, (1973), "On the gravitational field of a massless particle", JETP, 1973, Vol. 37, No. 6, p. 953

[351] W. B. Bonnor, (1969), "The Gravitational Field of Light", Comm. Math. Phys. 13, 163-174.

[352] Venzo de Sabbata, C Sivaram, (1994), "Spin and Torsion in Gravitation", World Scientific.

[353] R. T. Hammond, (2010), "The necessity of torsion in gravity", International Journal of Modern Physics D Vol. 19, No. 14 (2010) 2413-2416.

[354] A. Trautman, (1973), "Spin and Torsion May avert Gravitational Singularities", Nature Physical Science, Vol. 142, 7-8.

[355] E.J. Beggs, S. Majid, (2009), "*-Compatible Connections in Noncommutative Riemannian Geometry", https://arxiv.org/pdf/0904.0539v2.

[356] Suraj N. Gupta, (1954), "Gravitation and Electromagnetism", Phys. Rev., Vol 96, N. 6.

[357] S. Weinberg, (1965), "Photons and. Gravitons in Perturbation Theory: Derivation of Maxwell's and Einstein's Equations", Phys. Rev., Vol 138, N. 4B.

[358] Ashtekar A. and Ranjeet S. Tate, (1991), "Lectures on nonperturbative canonical gravity", World Scientific Pub Co Inc.

[359] Flip Tanedo, (2011), "Helicity, Chirality, Mass, and the Higgs", Quantum Diaries, https://www.quantumdiaries.org/2011/06/19/helicity -chirality-mass-and-the-higgs/. Retrieved on November 10, 2019.

[360] Wikipedia, "Belinfante-Rosenfeld stress-energy tensor", https://en.wikipedia.org/wiki/Belinfante\%E2\%80\%9 3Rosenfeld stress $\% \mathrm{E} 2 \% 80 \% 93$ energy tensor. Retrieved December 12, 2019. 
[361] The reference frame, (2019), "No global symmetries in QG 2019", https://motls.blogspot.com/2019/05/no-globalsymmetries-in-qg-2019.html. Retrieved on December 11, 2019.

[362] Daniel Harlow, Hirosi Ooguri, (2018), "Symmetries in quantum field theory and quantum gravity", https://arxiv.org/pdf/1810.05338v2.

[363] Lee C. Loveridge, (2004), "Physical and Geometric Interpretations of the Riemann Tensor, Ricci Tensor, and Scalar Curvature", https://arxiv.org/pdf/grqc/0401099v1.

[364] John M. Lee, (2018), "Introduction to Riemannian Manifolds", Springer.

[365] Rosen, Nathan (1940). "General Relativity and Flat Space. I". Physical Review, 57 (2): 147-150.

[366] Zwiebach, Barton (2003). "A First Course in String Theory". Cambridge University Press.

[367] Edmund Bertschinger, (2002), "Symmetry Transformations, the Einstein-Hilbert Action, and Gauge Invariance", Physics 8.962, MIT.

[368] Bronstein M, (1936), "Quantentheorie schwacher Gravitationsfelder", Phys. Z. Sowjetunion 9 ,140157.

[369] Bronstein M P, (1936), "Kvantovanie gravitatsionnykh voln" (Quantization of Gravitational Waves), Zh. Eksp. Tear. Fiz. 6, 195.

[370] Paul Sutter, (2020), "Is string theory worth it?", https://www.space.com/is-string-theory-worthit.html. Retrieved on May 05, 2020.

[371] Tamiaki Yoneya, (1974), "Connection of Dual Models to Electrodynamics and Gravidynamics", Progress of Theoretical Physics, Vol. 51, No. 6.

[372] J. Scherk and J. H. Schwarz, (1974), "Dual Models for Non-Hadrons", Nuclear Physics BS1 (1974) I18144.

[373] Yaakov Y. Fein et al. (2019), "Quantum superposition of molecules beyond $25 \mathrm{kDa}$ ", Nature Physics.

[374] C. F. Ockeloen-Korppi, E. Damskagg, J.-M. Pirkkalainen, A. A. Clerk, F. Massel, M. J. Woolley, M. A. Sillanpaa, (2017), "Entangled massive mechanical oscillators", https://arxiv.org/pdf/1711.01640v1.

[375] Claudia de Rham, (2014), "Massive Gravity", https://arxiv.org/pdf/1401.4173v2.

[376] Kurt Hinterbichler, (2011), "Theoretical Aspects of Massive Gravity", https://arxiv.org/pdf/1105.3735v2

[377] Fierz, M. and Pauli, W., (1939), "On relativistic wave equations for particles of arbitrary spin in an electromagnetic field", Proc. Roy. Soc. Lond., A173, 211-232.

[378] Stephen W. Hawking, Malcolm J. Perry, Andrew Strominger, (2016), "Soft Hair on Black Holes", https://arxiv.org/pdf/1601.00921v1.

[379] Eddington, A. (1935). "The Nature of the Physical World". MacMilan.

[380] Wikipedia, "Rotating black hole", https://en.wikipedia.org/wiki/Rotating black hole. Retrieved on February, 17, 2019.

[381] Wikipedia, "Charged black hole", https://en.wikipedia.org/wiki/Charged_black_hole. Retrieved on February, 17, 2019.

[382] Jacob D. Bekenstein, (1997), "Quantum Black Holes as Atoms", https://arxiv.org/pdf/gr-qc/9710076v2.

[383] Radhakrishnan C. Nair, 92006), "Photon as a black hole", SFIN A 1 (2007) 321-326.

[384] A.Burinskii, (2007), "Kerr Geometry as Space-Time Structure of the Dirac Electron", https://arxiv.org/pdf/0712.0577v1.

[385] Burinskii, Alexander, (2008), "The Dirac-KerrNewman electron", https://arxiv.org/pdf/0507109v4.

[386] J. Makela, P. Repo, M. Luomajoki, J.Piilonen, (2000), "Quantum-mechanical model of the Kerr-Newman black hole", https://arxiv.org/pdf/gr-qc/0012055v1.

[387] Wikipedia, "Black hole electron", https://en.wikipedia.org/wiki/Black_hole_electron. Retrieved on April 13, 2020.

[388] Richard G. Milner, (2013), "A Short History of Spin", https://arxiv.org/pdf/1311.5016v1.

[389] Wikipedia, "Spin (physics)", https://en.wikipedia.org/wiki/Spin_(physics). Retrieved on May 7, 2020.

[390] Jean-Marc Levy-Leblond, (1967), "Nonrelativistic Particles and Wave Equations", Commun. math. Phys. 6.

[391] von W. Gordon, (1928), "Der Strom der Diracschen Elektronentheorie", Z. Phys., 50, 630 (1928).

[392] F.J. Belifante, (1939), "On the spin Angular Momentum of Mesons", Physica, VI, N9.

[393] L. Rosenfeld, (1940), "On the energy-momentum tensor", Mem. Acad. Royal Belg., 18, N6.

[394] H.C. Ohanian, (1984), "What is spin?", Am. J. Phys., $54,(6), 1986$.

[395] Edouard Manoukian, (2016), "Quantum Field Theory I: Foundations and Abelian and Non-Abelian Gauge Theories", Springer. 
[396] Aneesh Manohar and Howard Georgi, (1984), "Chiral Quarks and The Non-Relativistic Quark Model", Nuclear Physics B234 189-212.

[397] W. Rory Coker, "Chiral Symmetry Breaking!", https://web2.ph.utexas.edu/coker2/index.files/chiralsb.htm. Retrieved, November 10, 2019.

[398] David Tong, (2018), "Lectures on Gauge Theory", http://www.damtp.cam.ac.uk/user/tong/gaugetheory. html. Retrieved on November 10, 2019.

[399] S. Weinberg, (1964), "Derivation of Gauge Invariance and The Equivalence Principle from Lorentz Invariance and the S-Matrix", Phys. Letters, Vol 9, N. 4.

[400] M.C. Gonzalez-Garcia and M. Yokoyama, (2019), "14. Neutrino Masses, Mixing, and Oscillations", in M. Tanabashi et al. (Particle Data Group), Phys. Rev. D 98, 030001 (2018) and (2019) update.

[401] Sidney Coleman, (1977), "Fate of the false vacuum: Semiclassical theory", Phys. Rev. D 15, 2929.

[402] Curtis G. Callan, Jr. and Sidney Coleman, (1977), "Fate of the false vacuum. II. First quantum corrections", Phys. Rev. D 16, 1762.

[403] The reference frame, (2011), "Bubble of nothing and other catastrophes", https://motls.blogspot.com/2011/10/bubble-ofnothing-and-other.html. Retrieved on April 14, 2020.

[404] J.R. Espinosa, G. Giudice, A. Riotto, (2017), "Cosmological implications of the Higgs mass measurement", https://arxiv.org/pdf/0710.2484v1.

[405] Carlos Mergulhao Jr., (1995), "Neutrino Helicity Flip in a Curved Space-time", General Relativity and Gravitation volume 27, pages 657-667.

[406] Steven Weinberg, (2020), "Models of Lepton and Quark Masses", https://arxiv.org/pdf/2001.06582v1.

[407] J. Ambjorn, J. Jurkiewicz, R. Loll, (2005), "Reconstructing the Universe", https://arxiv.org/pdf/hep-th/0505154v2.

[408] R. Loll, J. Ambjorn, J. Jurkiewicz, (2005), "The Universe from Scratch", https://arxiv.org/pdf/hepth/0509010v3.

[409] J. Ambjorn, J. Jurkiewicz, R. Loll, (2006), "Quantum Gravity, or The Art of Building Spacetime", https://arxiv.org/pdf/hep-th/0604212v1.

[410] Wikipedia, "Numerical relativity", https://en.wikipedia.org/wiki/Numerical_relativity. Retrieved on November 7, 2019.

[411] Aidan Randel-Conde, (2014), "The (seemingly) fractal nature of matter", Quantum Diaries, https://www.quantumdiaries.org/2014/05/27/the- seemingly-fractal-nature-of-matter/. Retrieved on November 11, 2019.

[412] Morag Scrimgeour, etal., (2012), "The WiggleZ Dark Energy Survey: the transition to large-scale cosmic homogeneity", https://arxiv.org/pdf/1205.6812v2.

[413] Paul H. Coleman Luciano Pietroneroc, (1992), "The fractal structure of the universe", Physics Reports, Volume 213, Issue 6.

[414] Morag Scrimgeour, (2013), "Large-scale homogeneity vs. smallscale inhomogeneity: testing $\Lambda C D M$ with large scale structure", Lawrence Berkeley National Laboratory, 18 January 2013, https://cosmology.lbl.gov/talks/Scrimgeour_13.pdf. Retrieved on May 12, 2020.

[415] Laurent Notalle, (1993), "Fractal Space-Time and Microphysics: Towards a Theory of Scale Relativity", World Scientific Publishing.

[416] Laurent Nottale, (2011), "Scale Relativity and Fractal Space-Time: A New Approach to Unifying Relativity and Quantum Mechanics", World Scientific Publishing.

[417] Lucien Hardy, (1993), "Nonlocality for Two Particles without Inequalities for Almost All Entangled States", Phys. Rev. Letters, Vol 71, N 11.

[418] Mohamed S. El Naschie, (2011), "Quantum Entanglement as a Consequence of a Cantorian Micro Spacetime Geometry", Journal of Quantum Information Science, 2011, 1, 50-53.

[419] M.S. El Naschie, (2009), "The theory of Cantorian spacetime and high energy particle physics (an informal review)", Chaos, Solitons and Fractals 41 (2009) 2635-2646.

[420] Gianluca Calcagni, (2016), "ABC of multi-fractal spacetimes and fractional sea turtles", Eur. Phys. J. C, 76:181.

[421] Ho, Mae-Wan and Vitiello, Giuseppe. (2015). "Is Spacetime Fractal and Quantum Coherent in the Golden Mean?", Global Journal of Science Frontier Research. 15. 61-80.

[422] Sergiu I. Vacaru, "Fractional Dynamics from Einstein Gravity, General Solutions, and Black Holes", https://arxiv.org/pdf/1004.0628v1.

[423] El-Nabulsi Ahmad Rami, (2009), "Fractional Lagrangian Formulation of General Relativity and Emergence of Complex, Spinorial and NonCommutative Gravity", International Journal of Geometric Methods in Modern Physics, Vol. 6, No. 1 (2009) 25-76.

[424] Alireza K. Golmankhaneh, xiao-Jun Yang and D. Baleanu, (2015), "Einstein Field Equations Within Local Fractional Calculus", Rom. Journ. Phys., Vol 60, Nos. 1-2, 2015. 
[425] Gianluca Calcagni, (2011), "Geometry of fractional spaces", https://arxiv.org/pdf/1106.5787.

[426] Gianluca Calcagni, (2011), "Geometry and field theory in multi-fractional spacetime", https://arxiv.org/pdf/1107.5041v4.

[427] Ji-Huan He, (2018), "Fractal calculus and its geometrical explanation", Results in Physics 10 (2018) 272-276.

[428] Gianluca Calcagni, (2018), "Towards Multifractional Calculus", Front. Phys., 21 June 2018.

[429] Alireza K. Golmankhaneh and D. Baleanu, (2015), "Calculus on Fractals", in "Fractional Dynamics", Edited by: Carlo Cattani, Hari M. Srivastava and xiao-Jun Yang, De Gruyter, 2015.

[430] S. S. Bay n, (2012), "Time Fractional Schrodinger Equation; Fox's H-functions and the Effective Potential", https://arxiv.org/pdf/1103.3295v4.

[431] Selcuk S. Bayin, (2012), "On the Consistency of the Solutions of the Space Fractional Schrodinger Equation", J. Math. Phys. 53, 042105

[432] Trifce Sandev, Irina Petreska, Ervin K. Lenzi, (2016), "Effective Potential from the Generalized TimeDependent Schrodinger Equation", Semantic Scholar.

https://pdfs.semanticscholar.org/f19a/65925e1204c3 ab1aca2fce3324d55903d979.pdf

[433] Johan L.A. Dubbeldam, Zivorad Tomovski and Trifce Sandev, (2015), "Space-Time Fractional Schrodinger Equation With Composite Time Fractional Derivative", Fractional Calculus and Applied Analysis, October 2015.

[434] El-Nabulsi Ahmad Rami, (2008), "Fractional Field Theories From Multi-Dimensional Fractional Variational Problems", International Journal of Geometric Methods in Modern Physics, Vol. 5, No. 6 (2008) 863-892.

[435] Nikolai Laskin, (1999), "Fractional Quantum Mechanics and Levy Path Integrals", https://arxiv.org/pdf/hep-ph/9910419v2.

[436] Nick Laskin, (2008), "Fractional Quantum Mechanics", https://arxiv.org/pdf/0811.1769v1.

[437] Gianluca Calcagni, Giuseppe Nardelli, Marco Scalisi, "Quantum mechanics in fractional and other anomalous https://arxiv.org/pdf/1207.4473v2.

[438] Madhat Sadallah, Sami I. Muslih and Dumitru Baleanu, (2011), "Fractional Time Action And Perturbed Gravity", Fractals, Vol. 19, No. 2 (2011) 243-247.

[439] Shaowei Wang, (2007), "Generalized fractional Schrodinger equation with space-time fractional derivatives", Journal of Mathematical Physics 48, 043502 (2007)

[440] Alexander I. Nesterov, Lev. V. Sabinin, (2000), "Nonassociative geometry and discrete structure of spacetime", https://arxiv.org/pdf/hep-th/0003238v1.

[441] Alain Connes, (1994), "Noncommutative Geometry", Academic Press.

[442] Snyder, Hartland S., (1947), "Quantized SpaceTime", Phys. Rev. 71 ( 1947) 38; 72 ( 1947) 68.

[443] R V Mendes, (1994), "Deformations, stable theories and fundamental constants", Journal of Physics A: Mathematical and General, Volume 27, Number 24.

[444] R. Vilela Mendes, (2017), "The geometry of noncommutative https://arxiv.org/pdf/1710.06756v1.

[445] R. Vilela Mendes, (2019), "Space-time: Commutative or noncommutative?", https://arxiv.org/pdf/1901.01613v2.

[446] Dionigi M. T. Benincasa, Fay Dowker, Bernhard Schmitzer, (2010), "The Random Discrete Action for 2-Dimensional https://arxiv.org/pdf/1011.5191v2.

[447] Wikipedia, "Weyl's tile argument", https://en.wikipedia.org/wiki/Weyl $\% 27$ s tile argum ent. Retrieved on Dec. 17, 2019.

[448] Jean Paul Van Bendegem, (1987), "Zeno's Paradoxes and the Tile Argument", Philosophy of Science, Vol. 54, No. 2 (Jun., 1987), pp. 295-302.

[449] Andreas Kempf, (1999), "A Generalized Shannon Sampling Theorem, Fields at the Planck Scale as Bandlimited Signals", https://arxiv.org/pdf/hep$\underline{\text { th/9905114v2. }}$

[450] Hooft, Gerard 't, (2016), "How quantization of gravity leads to a discrete space-time", J. Phys.: Conf. Ser. 701012014

[451] Georgios Moschidis, (2018), "A proof of the instability of AdS for the Einstein-massless Vlasov system", https://arxiv.org/pdf/1812.04268v1.

[452] Wikipedia, "Inflation (cosmology)", https://en.wikipedia.org/wiki/Inflation (cosmology). Retrieved on March 31, 2019.

[453] Shinji Tsujikawa, (2003), "Introductory review of cosmic inflation", https://arxiv.org/pdf/hep$\mathrm{ph} / 0304257 \mathrm{v} 1$.

[454] J. Alberto Vázquez, Luis E. Padilla, Tonatiuh Matos, "Inflationary Cosmology: From Theory to Observations", https://arxiv.org/pdf/1810.09934v2.

[455] Wikipedia, "Inflaton", https://en.wikipedia.org/wiki/Inflaton. Retrieved on November 11, 2019. 
[456] Nathan Seiberg, Edward Witten, (1999), "String Theory and Noncommutative Geometry", https://arxiv.org/pdf/hep-th/9908142v3.

[457] Wikipedia, "Big Bounce", https://en.wikipedia.org/wiki/Big_Bounce. Retrieved May 12, 2019.

[458] Shany Danieli, Pieter van Dokkum, Charlie Conroy, Roberto Abraham, and Aaron J. Romanowsky, (2019), "Still Missing Dark Matter: KCWI Highresolution Stellar Kinematics of NGC1052-DF2", The Astrophysical Journal Letters, Volume 874, Number 2.

[459] R. Loll, (2019), "Quantum Gravity from Causal Dynamical Triangulations: A Review", https://arxiv.org/pdf/1905.08669v1.

[460] Wikipedia, "Yang-Mills existence and mass gap", https://en.wikipedia.org/wiki/Yang-

Mills existence and mass gap. Retrieved on December 18, 2019.

[461] Lucini, Biagio; Teper, Michael; Wenger, Urs, (2004). "Glueballs and k-strings in $\mathrm{SU}(\mathrm{N})$ gauge theories: calculations with improved operators", https://arxiv.org/pdf/hep-lat/0404008.

[462] Andrzej Dragan and Artur Ekert, (2020), "Quantum principle of relativity", New J. Phys. 22 (2020) 033038.

[463] Andrzej Dragan, (2008), "Why devil plays dice?", https://arxiv.org/pdf/0806.4875v1.

[464] Wikipedia, "Postulates of special relativity", https://en.wikipedia.org/wiki/Postulates of special relativity. Retrieved on March 11, 2020.

[465] Ignatowsky, W. v. (1910). "Einige allgemeine Bemerkungen iiber das Relativitatsprinzip". Physikalische Zeitschrift. 11: 972-976; See Wiki Sources, https://en.wikisource.org/wiki/Translation:Some_Ge neral Remarks on the Relativity Principle. Retrieved on March 11, 2020.

[466] A. A. Grib, W. A. Rodrigues, (1999), "Nonlocality in Quantum Physics", Springer.

[467] Jed Brody, (2020), "Quantum Entanglement", The MIT Press.

[468] Daniel Cavalcanti, Fernando G.S.L. Brandao, Marcelo O. Terra Cunha, (2005/2013), "Are all maximally entangled states pure?", https://arxiv.org/pdf/quant-ph/0505121v7.

[469] Jacques Colin, Roya Mohayaee, Mohamed Rameez, Subir Sarkar, (2018), "Evidence for anisotropy of cosmic https://arxiv.org/pdf/1808.04597v3.

[470] K. Migkas, G. Schellenberger, T. H. Reiprich, F. Pacaud, M. E. Ramos-Ceja, L. Lovisari, (2020),
"Proving cosmic isotropy with a new $\mathrm{x}$-ray galaxy cluster sample thorugh the LX -T scaling relation", https://arxiv.org/pdf/2004.03305v1.

[471] J. A. Wheeler, "Geons", Phys. Rev. 97, 511 (1955).

[472] Wheeler, John Archibald; Ford, Kenneth Wilson (2010). "Geons, black holes, and quantum foam: a life in physics". New York: W. W. Norton \& Company.

[473] Kong, J., Jimenez-Martinez, R., Troullinou, C. et al., (2020), "Measurement-induced, spatially-extended entanglement in a hot, strongly-interacting atomic system". Nat Commun 11, 2415.

[474] Wikipedia, "Cosmological constant problem", https://en.wikipedia.org/wiki/Cosmological_constant problem. Retrieved on May 26, 2020.

[475] Nima Arkani-Hamed, Lubos Motl, Alberto Nicolis, Cumrun Vafa, (2006), "The String Landscape, Black Holes and Gravity as the Weakest Force", https://arxiv.org/pdf/hep-th/0601001v2.

[476] Leonard Susskind , (1995), "Trouble For Remnants", https://arxiv.org/pdf/hep-th/9501106v1.

[477] Clifford Cheung, Junyu Liu, Grant N. Remmen, (2018), "Proof of the Weak Gravity Conjecture from Black Hole Entropy", https://arxiv.org/pdf/1801.08546v3.

[478] Garrett Goon and Riccardo Penco, (2020), "Universal Relation between Corrections to Entropy and Extremality", Physical Review Letters, 124, 101103 (2020)

[479] Wikipedia, "Black hole thermodynamics", https://en.wikipedia.org/wiki/Black hole thermodyn amics. Retrieved on March 20, 2019.

[480] Esteban Castro-Ruiz and Flaminia Giacomini and Alessio Belenchia and Caslav Brukner, (2020), "Quantum clocks and the temporal localisability of events in the presence of gravitating quantum systems", Nature Communications, (2020) 11:2672.

[481] Paul Langacker (2012), "Grand Unification",Scholarpedia, $\quad$ 7(10):11419, http://www.scholarpedia.org/article/Grand_unificatio n. Retrieved on May 31, 2020.

[482] nLab, (2020), "proton decay", https://ncatlab.org/nlab/show/proton+decay. Retrieved on May 31, 2020.

[483] Michael R. Wilczynska, John K. Webb, Matthew Bainbridge, Sarah E. I. Bosman, John D. Barrow, Robert F. Carswell, Mariusz P. Dabrowski, Vincent Dumont, Ana Catarina Leite, Chung-Chi Lee, Katarzyna Leszczynska, Jochen Liske, Konrad Marosek, Carlos J.A.P. Martins, Dinko Milakovic, Paolo Molaro, Luca Pasquini, (2020), "Four direct measurements of the fine-structure constant 13 billion years ago", https://arxiv.org/pdf/2003.07627v1. 
[484] Adler S.L., (2004), "Quantum theory as an emergent phenomenon", Cambridge University Press.

[485] G. 't Hooft, (1990), "The Black Hole Interpretation of String Theory", Nuclear Physics B335 (1990) 138154.

[486] Mario J. Neves, Everton M. C. Abreu, (2012), "Path integral formalism in a Lorentz invariant noncommutative https://arxiv.org/pdf/1206.4065v1.

[487] S. Doplicher, K. Fredenhagen and J. E. Roberts, (1994), "Spacetime quantization induced by classical gravity", Phys. Rev. B 331 (1994) 33.

[488] Sergio Doplicher, Klaus Fredenhagen and John E. Roberts, (1994), "The Quantum Structure of Spacetime at the Planck Scale and Quantum Fields", Commun. Math. Phys. 172, 187 -220 (1995).

[489] Markku Antero Oksanen, (2008), "Noncommutative Gravitation as a Gauge Theory of Twisted Poincare Symmetry", Thesis, Helsingin Yliopisto Fysikan Laitos, Finland.

[490] Francis Heylighen, (2018), "Entanglement, symmetry breaking and collapse: correspondences between quantum and self-organizing dynamics", ECCO Working paper, 2018-03, revised version for: Foundations of Science.

[491] J. Eisert, M. Cramer, M.B. Plenio, (2008), "Area laws for the entanglement entropy - a review", https://arxiv.org/pdf/0808.3773v4.

[492] Joon Hyeop Lee, Mina Pak, Hyunmi Song, Hye-Ran Lee, Suk Kim, Hyunjin Jeong, (2019), "Mysterious Coherence in Several-Megaparsec Scales Between Galaxy Rotation and Neighbor Motion", https://arxiv.org/pdf/1908.10972v1.

[493] Han-Ying Guo, Chao-Guang Huang, Yu Tian, Zhan xu, Bin Zhou, (2006), "Snyder's Quantized Spacetime and De Sitter Special Relativity", https://arxiv.org/pdf/hep-th/0607016v2.

[494] Wikipedia, "Stress-energy tensor", https://en.wikipedia.org/wiki/Stress-energy tensor. Retrieved on December 12, 2019.

[495] Eemeli Annala, Tyler Gorda, Aleksi Kurkela, Joonas Nattila and Aleksi Vuorinen, (2020), "Evidence for quark-matter cores in massive neutron stars", Nature Physics, https://www.nature.com/articles/s41567020-0914-9.

[496] Kansas State University, (2020), "Study finds that patterns formed by spiral galaxies show that the universe may have a defined structure", https://phys.org/news/2020-06-patterns-spiralgalaxies-universe.html. Retrieved on June 2, 2020.

[497] Leticia F. Cugliandolo, Vivien Lecomte, Frederic Van Wijland, (2018), "Building a path-integral calculus: a covariant discretization

approach", https://arxiv.org/pdf/1806.09486v3.

[498] Robert A Flatt, (2020), "A Resolution of the Proton Radius Puzzle", https://doi.org/10.6084/m9.figshare.12401054 Retrieved June 5, 2020

[499] M. Tanabashi et al. (Particle Data Group), (2018), "The Review of Particle Physics", Phys. Rev. D 98, 030001 (2018).

[500] A. K. F. Val Baker, (2020), " The puzzle of the proton radius", https://doi.org/10.31219/osf.io/myfxh .

[501] Wikipedia, "Free neutron decay", https://en.wikipedia.org/wiki/Free_neutron_decay. Retrieved on June 5, 2020

[502] Schwartz, M.D., (2013), "Quantum Field Theory and the Standard Model", Cambridge University Press.

[503] Kazuo Fujikawa, (2009), "Quantum anomalies and some recent developments", https://arxiv.org/pdf/0902.2066v1.

[504] Kazuo Fujikawa, Hiroshi Suzuki, (2014), "Path Integrals and Quantum Anomalies", Oxford University Press, USA; Reprint edition (January 21, 2014).

[505] Steven Weinberg, (1981), " The Decay of the Proton", Scientific American, Vol. 244, No. 6 (June 1981), pp. 64-75.

[506] Hyperphysics, "Protons and neutrons", http://hyperphysics.phyastr.gsu.edu/hbase/Particles/proton.html. Retrieved on June 5, 2020.

[507] R. Aldrovandi, G. E. A. Matsas, S. F. Novaes, D. Spehler, (1994), " Fermion Helicity Flip in Weak Gravitational Fields", https://arxiv.org/pdf/grqc/9404018v1.

[508] Soumitra SenGupta, Aninda Sinha, (2001), " Fermion helicity flip by parity violating torsion", https://arxiv.org/pdf/hep-th/0102073v2.

[509] Anindya Datta, Emidio Gabrielli, BarbaraMel, (2003), "Violation of angular momentum selection rules in quantum gravity", Physics Letters B 579 (2004) 189199.

[510] Julian Sonner, (2013), "Holographic Schwinger Effect and the Geometry of Entanglement", https://arxiv.org/pdf/1307.6850v3.

[511] Steven D. Bass, (2004), "Anomalous commutators and electroweak baryogenesis", https://arxiv.org/pdf/hep$\mathrm{ph} / 0403219 \mathrm{v} 1$.

[512] Arnold Neumaier, (2016), "Learn the Physics of Virtual Particles", https://www.physicsforums.com/insights/physicsvirtual-particles/. Retrieved on September 1, 2019. 
[513] Stephane Maes, (2020-2022), "Web Site Tracking all Publications around the Multi-fold universe", Navigation page listing all papers. https://shmaesphysics.wordpress.com/shmaesphysics-site-navigation/.

[514] Stephane H Maes, (2021), "The Multi-fold Theory: A synopsis", viXra:2112.0144v1, https://shmaesphysics.wordpress.com/2021/12/24/th e-multi-fold-theory-a-synopsis-so-far-v2-end-of2021/, December 24, 2021. Note that additional links will always be available at https://shmaesphysics.wordpress.com/2021/05/03/th e-multi-fold-theory-a-synopsis-so-far/ to track the latest and interim versions of the synopsis, as they may be published under different tittle or URL/publication numbers.

[515] Stephane H Maes, (2022), "Understanding the Multifold theory principles and the SM_G", https://shmaesphysics.wordpress.com/2022/03/11/un derstanding-the-multi-fold-theory-principles-andthe-sm_g/, March 11, 2022.

[516] Stephane H. Maes, (2022), "What is the Multi-fold Theory? Its Main Characteristics in a Few Words", vixra:2207.0172v1, https://shmaesphysics.wordpress.com/2022/07/28/w hat-is-the-multi-fold-theory-its-main-characteristicsin-a-few-words/, July 28, 2022.

[517] Stephane H Maes, (2022), "Deriving the Multi-fold Theory from General Relativity at Planck scale", https://shmaesphysics.wordpress.com/2022/02/22/de riving-the-multi-fold-theory-from-general-relativityat-planck-scale/, February 22, 2022.

[518] Raphael Bousso, (2002), "The holographic principle", https://arxiv.org/abs/hep-th/0203101. 


\section{ENDNOTES}

1. Think à la SUSY/Super symmetry/Super gravity/Super strings. Today they are threatened by, for example, the absence of observations of SUSY particles (aka super partners) at LHC, and other accelerators, the absence of observations of proton decay $[239, \underline{240}, \underline{248}, \underline{236}]$, as well as the possibly even bigger problem of unobserved magnetic monopoles $[\underline{321}, \underline{256}, \underline{341]}$, predicted by GUT, supersymmetry theories, supergravity and superstrings.

2. Indeed, magnetic monopoles probably don't exist, simply because gravity seems to break the electromagnetism duality [342]. We will revisit later, once we can safely argue the validity of a semi classical approach.

3. Meaning that physics models remain as applicable in $U_{M F}$ as in $U_{\text {real }}$; unless when said explicitly otherwise.

4. Besides also the issues of background independence of GR vs. background dependence of QFT.

5. However, it is known that every physical theory, and model, do not necessarily come with a known Lagrangian, Action or Hamiltonian. Exceptions with no, or multiple Lagrangian are encountered in high energy, high interaction or emergent/induced/effective theories $[\underline{62}, \underline{63}, \underline{322}]$, as well as in phenomenological theories, and phase transition models. Think of the Ising model, for example.

6. We will show that our discrete model of spacetime, and of particles, could in fact give a hint of why Actions exist, and are extremized through the natural existence of a multi-paths formalism, like the Path Integrals in $U_{M F}$.

7. Something that, from the onset, prevents QFT from explicitly capturing a particle's entanglement vs. just modelling statistical bulk, or surface entanglement, and entanglement entropy (also known as Von Neumann entropy) related to the density operator, mixed states, and entanglement Hamiltonians [푸 $\underline{241}, \underline{242}]$.

8. Because in $U_{M F}$, we have a discrete spacetime, torsion and expansion tendencies.

9. In fact, we can argue that we derive them without postulating modifications/perturbation of the Action, or perturbation of the metric in GR.

10. As well as for example the problems of proton decay, and even more problematic of magnetic monopoles.

11. By this, we mean that the EPR paradox is resolved by ensuring that two spacetime points, where EPR entangled particles are located, are actually the same point for some newly enabled paths so that indeed instantaneous exchanges result from having exchanges over such these paths (they are the same entity there). In the Bell inequality/EPR parlance, these new paths allow explicitly the measurement of one EPR entangled particle to influence the measurement of the other particle at another location.

12. For now, imagine a wormhole where paths can meet. Note we do not impose the Physics folds (e.g., dynamics and kinematics or embedding space), and so we do not assume, nor will we derive, wormholes. It is just a way to imagine, and it is a possibility of the model.

13. Such a feat in our view should be evaluated in the light of the history of strings, born in the 70's based on the apparition of graviton-like particles; even if that became obvious the moment that the Nambu-Goto action was introduced: it imposed the same area extremization as the Hilbert Einstein Action [139]. In the subsequent revolution frenzies in the 80's then in the 90's, this apparition remained the single most important argument put forward as justification for the work, along with the satisfaction of proofs (or conjectures depending on to who you ask) of renormalizability and absence of divergences.

14. Schwinger and Freeman Dyson merit also recognition [244, 245], with equivalent formulations.

15. See 5 for exceptions: it is not always the case as has been recently more systematically observed.

16. Yet gauge fields like Yang Mills of QCD have only been successfully constructed for spacetime dimensions smaller than 4 (see for example $[\underline{65}, \underline{67}]$ ). Many have tried to resolve the 4-dimensional use case and promising new approaches based on complex Path Integral seems to narrow down the problem [68]. Our discussion in section 9.7 may lead to another approach. Complex hints at discrete fractional spacetime as discussed later on. 
17. They amount to requiring: i) Covariance, ii) Locality (the speed of transfer of information is upper bounded by c), iii) Observability, iv) Vacuum uniqueness (i.e. it is invariant under time translations up to scalar multiples) are satisfied along for a set fundamental principles [70, 71, 69] that roughly imply: a) Analyticity, b) Euclidicity (possibility to work with complex variable when transforming $\mathrm{t} \rightarrow-$ itl; and so statistical field duality), c) reflection positivity (related to possibility to observe and define unitary propagators), d) ergodicity (unique vacuum or probabilities having the same behavior averaged over time as averaged over the space of all the system's states in its phase space, i.e., Fourier transforms are possible and meaningful.

18. We will see later that, in a multi-fold universe, relativistic Paths Integral consider that paths passing outside the light cone (i.e. with a space like portion for a point on the path) are not allowed, and to be filtered out in $U_{M F}$. It is a different approach from the conclusions of [74, 339]. [339] arguments to justify counting these paths in QM and QFT, by analogy to other non-classical paths, met in two slits experiments, is in our view questionable: violating supra luminosity (and therefore causality; yes, the uncertainty principle allows walking back in time in perturbations, and therefore in Feynman diagrams. But that should not be true for larger paths!). It is quite different from allowing nonclassical paths through say a potential barrier, or a two slits experiment, where we know that non classical paths play a role (but these are not paths outside the light cone) [261, 340]. Yes, [339] finds corroboration with QFT and particle propagators, e.g., Feynman propagator, but these are computed with the same approximations.

19. At least for some paths, considered in the Path Integrals describing the system.

20. Interestingly, entangled (connected) black holes were also proposed in the ER=EPR conjecture [86]. [86] focused rather on the implications in AdS (Anti de Sitter Space)-A maximally symmetric spacetime with negative cosmological constant/constant negative curvature solution of Einstein's GR field equations, widely used because many complex problems are 'easier" to model in AdS spacetime instead of our universe widely believed not to be of negative curvature. We will encounter AdS again and again later and motivate, at the light of our approach and in the context of $U_{M F}$, its presence in many of other theories trying to address particle physics, field theories, superstrings and gravity - along with the trendy AdS/CFT correspondence. In relation to ER=EPR, it has also been hinted that Planck scale black holes may connect entangled particles [510], yet not explaining what happens at larger scales or identifying that this leads to gravity emergence.

21. Again, the analysis is focused on AdS, and it does not explore the implications of allowing paths contributing to the Path Integrals between the particles to traverse the black holes [328, 326, 327]. [86] refers to non-traversable wormholes. Some follow-up work looked at some consequences of ER=EPR approach [87, $\underline{88}, \underline{50]}$. If wormholes are not traversable, then they cannot satisfy the required features for folds in $U_{M F}$, as paths cannot be added. In our view this, is really the cusp of why ER=EPR has not been able to pursue the model to the next level as we provide here. Only solutions where the wormholes are traversable would be acceptable. Some works have considered when such options exist, unfortunately typically only for AdS spacetime, or for exotic matter (which would not be allowed path for non-exotic matter), which would also not be acceptable for Ureal. Wormholes in our spacetime (i.e. not AdS) present another challenge: they are especially hard to imagine because wormholes would be present anywhere (where there is entanglement), and arguably observable if they were to live in our spacetime (we do not observe any of them - yet they would have to link macroscopic distances in the spacetime of $U_{\text {real }}$, be traversable and can't require exotic matter to exchange $[\underline{89}, \underline{90}]$. Note that ER=EPR may avoid some of these problems with for example the argument that the entanglements of the black holes, via ER, is not observable because ER bridges can't be observed [87]. However, it does not address the other points. Arguments that full entanglement of the black holes would enable traversability (for some signal [91].), is in any case something that is aligned with our results, but not required. In this paper, we will show results without requiring an AdS spacetime.

22. and yet, it explains that the mechanism we create lives in $\operatorname{AdS}(5)$ with multi-folds that can encompass traversable wormholes, or connected black holes but do not assume them.

23. By the way, it is worth teasing the reader that we will recover a model of (entangled) micro (quasi) black holes in a space like $\operatorname{AdS}(5)$ tangent dual to $U_{\text {real }}$. The analogy to the ER=EPR conjecture, and its concretization/explanation in $U_{M F}$ is certainly eye opening; especially as it seems interesting that such similar concepts have been independently proposed, and that it may hint that our intuitive idea, although crazy, may not be that crazy. It also has significant implications for our next steps in the second part of the paper, and for many other models, and conjectures in Physics, including in particular the CFT/AdS correspondence conjecture, strings and the swampland (e.g. spoiler alert for the string supporters: In our framework, it's OK that strings would, or can, only live in AdS $(\times \mathrm{S} 5)$ (or (+1 more for Mtheory) spacetimes, if the universe expands per [94]; that is indeed where they have to live in our model, if they exist, and their existence is something that seems hinted in $U_{\text {real }}$ ); without starting from the Hilbert Einstein's Action behind Einstein's GR field equations. 
24. This will be discussed later. In general torsion will be null outside matter (meaning here fermions and bosons), but there is, or rather may be, an in-matter microscopic torsion contribution emerging from our model. Macroscopically it averages out.

25. The torsion that we will mention later, as predicted by our model, is not captured by the multi-folds as twisted spacetime, but as the result on the uncertainty about the folds (entry and exit points) vs. particles positions and momenta in $R_{B G}$.

26. This argument (avoiding particle creation or destruction in the folds) also motivates the geometry, kinematics and dynamics concretely proposed for the multi-folds for the events of interest, as discussed in an upcoming section.

27. On the other hand, we will argue that what we observe Today, with say EPR entanglement, is indeed something tractable and observed (at least unless if invalidated by experimentation, when trying falsification or validation of our model).

28. i.e., unitarity and information can be conserved.

29. On the other hand, superstring theory may care, and have some answers to this.

30. For our paper these events are only related to EPR entanglement, and disentanglement.

31. Yet, if we were to reject some of these behaviors, in some circumstances in the future, it might explain particular symmetry violations.

32. As well as in the many worlds view of the world [104], although it is radically different, and in a different context.

33. Indeed, uncertainty in spacetime position may result into apparent space-like or back-in-time moves.

34. Yet, not universally agreed upon.

35. Additional motivations will be given later, with an analysis motivating the action principle in Physics in $U_{M F}$ in the context of our discrete reconstruction of $U_{M F}$.

36. Remembering the hard partitioned tenancy, with one (multi-) fold instance per particle, which implies that no interaction with other particles (real or virtual) can take place in the folds; except at the end points, and with the Higgs.

37. Something not present, and in fact not desired, in Kaluza-Klein, or string theory.

38. In AdS, these could be wormholes/ER bridges [우, 90], or black holes, per the ER=EPR conjecture [86], or Wheeler's Geometrodynamics [332].

39. This is a fundamental difference with Kaluza-Klein, which adds a (5-D) Hilbert Einstein action to the extra dimension (or higher dimension if more compact dimensions are considered). It is well known that this latter approach results into new fields (e.g. they were thought to model Electromagnetism), and particles [254, 256], as well as, unfortunately, instabilities (e.g. [256, $\underline{333}])$.

40. We should probably remember, in this context, the work of V.Ignatowsky [464, 465] who showed that Lorentz transformations, and therefore an invariant speed limit, is contained in special relativity principles (without imposing the invariance of $c$ ). Deriving its value then requires electromagnetic considerations.

41. Indeed, as shown for example in [339], the propagation amplitude of a particle associated to a field is directly linked to the expectation value of the ground state at the different points, and hence impacts all field space like correlations and vacuum behaviors: if nothing propagates outside the light cone, no correlation takes place.

42. the latter two being field equations.

43. The measurement produced a nonlocal effect on the entire wave function [466]. Challenges with quantum measurement, and the associated wave function collapse, have led to different interpretations of quantum mechanics with in particular the Copenhagen interpretation vs. the Many-worlds [104], the Bohmian interpretation [101, 102, 466], and the decoherence story with spontaneous collapses etc. [336, 466] etc. It has also been argued that, maybe, wave functions are time symmetric, or transactional (with retarded (offer), and advanced (confirmation) wave function), to resolve the challenges in explaining the nonlocal aspects of the wave function collapse, and its implied non-reality, see for example $[\underline{338}, \underline{337}, \underline{335}]$. Transactional Quantum mechanics offers an interesting explanation for wave function collapses without the otherwise apparent violation of supra luminous limits (Another phenomenon challenging Relativity, like EPR entanglement, and that, who knows, may otherwise also warrant folds, mappings and events. We will not study this here. Watch [513] for papers on this.), which is no no more implied in transactional 
QM. Transactional QM is even compatible with perturbation-derived Feynman diagrams. So, for all purpose, and without going more into details, we assume that, in $U_{M F}$, it is possible one way or another (i.e. with one or the other of these interpretations), to address these other paradoxes, or puzzles, without requiring supra luminous events. That principle must remain unviolated in $U_{M F}$ !

44. the argument (for: $\left.x_{0}^{v}, t\right)$ designates that the fold is the evolution (at $\mathrm{t}$ ) of the fold created at $x_{0}^{\mu}\left(\mathrm{i} . \mathrm{e}\right.$. at $\left(t_{0}, x^{a}\right)$ ).

45. As allowed paths.

46. This diagram assumes without loss of generalities $\mathrm{f}$ for time (not just the final time of measurement as the behavior is also at any interim time. It is just that at $f$, measurement takes place, and the particles disentangle.) and $x_{0}^{\mu}=x_{C M}^{\mu}$.

47. This implies a special relationship between Hilbert spaces/state spaces and configuration spaces that we will revisit. The center of mass behavior is also confirmed when looking at the phase space [246].

48. For now, let's assume that $R_{B G}$ can be flat, or curved, without any loss of generality: the folds will be the same independently of the curvature (or even torsion) in $R_{B G}$. Intuitively, it is because the objectives of the mappings do not need such complexity; they just need to map to the particle's positions and movements. If the curvature affects those, then the mappings and fold evolution just need to be adjusted; nothing more.

49. More on this later.

50. This was also inspired by the original ideas of [98].

51. As the fold has itself evolved with the center of mass of the two particles.

52. We will discuss later the implications of equation (9), but, at a high level, it is the root of Einstein's equivalence principle.

53. We will get back to this; but, spoiler alert, it announces a mapping of the folds to gravitons, when the mechanism is itself quantized; something that we can only anticipate that it will happen at this stage of the reasoning. However, as the folds live outside the background spacetime of $U_{M F}$, by construction. it already hints that the graviton may not behave exactly as other particles, and that, by analogy to superstrings, it may be associated to closed entities outside $U_{M F}$ spacetime. Of course, as already mentioned earlier, there could be variations of our model where the folds could be wormholes within $U_{M F}$, or in "other dimensions". The reader can probably start guessing how all these models may relate to each other.

54. As well as symmetry breaking.

55. It might be possible to imagine folds with different entry points; however, our intuitive approach did not see value, or reasons, to do so, yet. We will revisit, in future works, if hierarchical entanglement would sometimes support such a model; but we believe that, as hierarchical entanglement always results of combinations of direct local interactions, it is not needed.

56. Unless different entry points to a fold were authorized. That is not our model for now or in this paper.

57. Later, we will discuss how to deal with QFT, and its space-like entanglements. Our spacetime reconstruction for $U_{M F}$ also discusses how spacetime entanglement can be modeled, and how inflation may result. In general, it is important to understand that, in $U_{M F}$, and for things happening post inflation, no attraction results between entangled spacelike regions or entities. This is a big difference from say $[\underline{125}, \underline{126}, \underline{133}, \underline{134}]$. It results from the strong no supra luminosity principle in $U_{M F}$.

58. As we will see later, it is the correct interpretation in $U_{M F}$, where spacetime is discrete.

59. Hence the dependency on energy, or mass.

60. The arbitrary translation step, in the proof, as in say [269], falls apart in $U_{M F}$.

61. As center of mass of entangled virtual particle/antiparticle pairs.

62. And so, we maintain that massive, and massless effects, as discussed later, are present and, at some point, may be detectable.

63. Setups where that would not be that case would create anisotropic attractive potentials, that could be seen as anisotropic gravity like effects. 
64. Not Rosen's bigravity [ $\underline{348}, \underline{347]}$. In fact, we would rather have a range of multi-gravity than just two. Although, interestingly, we will soon re-encounter some of the bases of Rosen's bigravity.

65. The fundamental mechanism is the multi-folds support of entanglement that results in recovering GR at large scales. At small scale, for massive gravity, the nature of the classic solution may or may not be well modelled: GR had no such concepts. That is not an issue for us: we also predict a (set of) massive gravity contributions at very small scales; but do not derive them as massive gravity was conventionally derived. The steps of generating curvature (Ricci scalar and tensor), and $V_{\text {eff }}$ may just be all what matters, rather than having to derive something expressed classically as GR is. In our model, multi-folds, viewed as gravitons, exist outside spacetime. So, it may simply be why modelling with traditional QFT, and GR linearization [377, 375] seems to always fail. Exploring these aspects are of future interest.

66. This is discussed in more details after.

67. $r$ is the distance between particle encountering the domain support of the mapping and the center of mass of the virtual particles, i.e. the physical massless, or massive, particle.

68. The explanation shows why spin may matter; yet it is not to be confused with spin gravity, or spin torsion couplings which is not what we discuss in this section. These couplings rather result from proposing invariant Lagrangian, that can be constructed when involving spinors [294, 290, 345].

69. However, it has been argued that torsion is needed theoretically, and could result from spin to ensure angular momentum conservation in the presence of gravity [288, 289, 353]. The Belinfante-Rosenfeld stress-energy momentum tensor $[\underline{360}, \underline{392}, \underline{393}]$, used to impose symmetry, captures the torsion in a bound (spin) current, leading to an effective energy momentum tensor that is conserved, and symmetric, for all purposes of classical and macroscopic GR. This is what is typically used, but it obfuscates the microscopic presence of torsion. The present paragraph shows that we reach similar conclusions: torsion can appear, and it is due to spin; some of this will be clarified as we discuss spin. relevance, and/or its inevitability and or inevitability is not definitive.

70. In fact, Feynman [323], and Weinberg [357], showed that spin-2 interactions, and respect of Lorentz symmetries imply GR (at large scales); not torsion. But it is important to remember that these derivations imposed symmetry, i.e., the former avoids, and disregards anti-symmetry, and the latter says nothing about it. But it is all good: it does not matter at large, classical, or semi classical scales. Note for completeness that Gupta's work [356], also linked spin-2 and GR derivation but, again, torsion less features are implicit and not discussed.

71. [353] presents some entry points. It is also to be note that strings predict torsion, as does LQG, and its spin network and spin foam derivatives, through Einstein Cartan-like actions and spin coupling of matter [294, 25, 83, 23, 295] Some particle proposals (e.g. dilation) exist, but they have not received much support, and seem to be contradicted by the Higgs mechanism [301].

72. Einstein Cartan theory [295, 294, 297], and Teleparallelism [271, 270], which trades curvature for torsion (no curvature exist; yet Teleparallelism is equivalent to GR) can avoid singularities and support big bounce solutions $[\underline{288}, \underline{289}, \underline{291}, \underline{296}, \underline{297}, \underline{271}, \underline{270}, \underline{292]}$.

73. that, at Planck scales, is, in our approach, also guaranteed by discreteness of spacetime.

74. Yet we are inclined to argue that the underlying mechanism will guarantee no singularity and big bounce solutions, even if it ended-up not creating torsion.

75. For weak interactions.

76. This is not in contradiction with the CPT theorem introduced in QFT for axiomatic QFT, enumerated Lagrangian examples or generic Lagrangian forms $[\underline{329}, \underline{330}]$. These work only for Minkowski spacetime. Indeed, $U_{M F}$ is quite different from Minkowski flat spacetimes i.e. $R_{B G}$. The CPT theorem exists also for QFT, without gravity, in a curved space with locality requirements [331], but again these are not satisfied by the multi-folds and mapping processes of our approach.

77. It may be worth noting [25] that the dependence on Ricci scalar is similar to Regge calculus situations where the Ricci scalar contributions on a lattice (Ponzano-Regge ansatz) leads to GR equations. It is the only curvature entity appearing in relation to the Hilbert Einstein Action, when working on a discrete lattice (besides directions of attraction). 
78. And while most boson (force carrier) propagators result into $(1 / r)$ potentials, possibly weighted by an exponential term if massive, the source of the $(1 / r)$ dependency is quite different in this paper. It is related to how $U_{M F}$, reacts and implements entanglement.

79. It may also also hint, in an interesting way, at why gravity is weak with gravitons living outside of spacetime, just like in string theory, gravitons are closed multi-folds (vs. strings) living outside spacetime, and like in the AdS/CFT correspondence. All these will be discussed again later. Yet these considerations line up to address nondivergence/renormalizability of our model, when captured with manipulable equations; even independently of handwaving a discrete spacetime. We will however question the strict weak Gravity Conjecture: at very small scales, it looks like the massive gravity contributions can match, and in fact meet the other interactions.

80. It is not that different from how one can interpret the way that Higgs boson in the sea of the vacuum gives mass to (charged) fermions by having them bumping (i.e. interacting with) into the Higgs boson [359].

81. This is to make sure that we add $R$, not $\sqrt{|R|}$, which is the result post integration across a bundle, and represents a surface invariant but just for the last at distance $r$; not something relevant to the spacetime background. Of course, it is interesting, and maybe confusing at the same time, that using $\sqrt{ }|R|$, would immediately recall the Hilbert Einstein Action. But it really isn't because we also have to involve the metric / Jacobian, and it would no more be easy to understand what happens next.

82. No double counting: it is one, or the other.

83. We know that torsion, and anticipated discrete spacetime should take care of that issue, when we reach that point of the model.

84. As an exercise, try to see that a stretched volume will generate gravity pressure as implied by GR, using the multifold scheme (of course, it will, if you use GR to derive it).

85. Albeit not necessarily with a GR dynamic, that is a priori not in our model.

86. Strings Actions include terms equivalent to Hilbert Einstein action resulting from the extremization of the world sheet area as does Hilbert Einstein area invariance on a GR manifold; but at the time of the basis of the work done by Veneziano, Virasoro and Shapiro, it was not yet understood that their underlying model matched strings, and it seemed more a chance discovery. The Nambu-Goto action would only appear in 1970 [366]. One could argue that any model that happens to include Hilbert Einstein Action variations will most probably introduce gravitons, no matter what that model aims to achieve. Extremizing the area of the world sheet of a string is really just what makes the graviton appears, and it becomes obvious once it is understood that the model, pioneered by Veneziano, Virasoro and Shapiro as amplitude matches to Hadron scattering results, could model strings with word sheet area extremization. Watch [513] for more details.

87. When we discuss quantization, and discretization, these approximations and assertions will be better motivated. As we are not yet there, the uncertainty principle is our savior for now.

88. Again, even if surprising, this is not unheard of. The curvature is a reflection of the evolution of the metric field. Einstein himself considered curvature as a way to imagine gravity effects. After all, we know that the work on Teleparallel gravity initiated by Einstein leads to a universe without curvature, and only torsion, and forces induced by torsion, and yet it is totally equivalent to GR [271, 270].

89. This also relates, and offers a different perspective, to what it means to perturb the curvature of spacetime (which results into perturbation of the metrics). It is typically a preamble to attempts to quantizing GR, encountering gravitons, and / or to introduce strings, or supersymmetries, to reduce some of the divergences introduce by such attempts [23]. Typically, it includes adding dimensions to spacetime, and modifying the Hilbert-Einstein Action to achieve such cancellation at higher orders.

90. Because of the need to model through them the back reaction, when the background is fixed, as in QFT, and superstring theory.

91. We have discussed already how that is actually handled, and eliminated, in $U_{M F}$. Clearly, full and rigorous analysis of the implications on axiomatic/reconstructive QFT, as well as impact on the events and probabilities computed with QFT would be of great interest; even if we believe that the effect is limited; it the sense that consistency and reconstruction remain valid in $U_{M F}$. 
92. Of course, this is the flip view of what is usually invoked to link these domains which rather refers to excitation network analogies between quantum field in spacetime, and vibrations in crystal / solid structures.

93. With particles themselves resolved with, and defined by the recipes above.

94. Albeit these can work for non-maximally entangled states; but for well-defined particles...

95. The need to model particles, and EPR entanglement, also applies to reconstructive quantum gravity approaches. But it is not as directly related to the challenges encountered with conventional QFT, that we have discussed here, so far.

96. We keep on adding these last words, because theories like QFT, or even strings, are still not the fundamental theories of nature (at least in their current form). Also, in $U_{M F}$, Quantum Physics is not the same at Planck scale Physics, as we will discuss later.

97. And why gravity is the weakest interaction at semi classical or classical scales due to the main effects occurring outside spacetime. The story is more complicated at smaller scales, and we will discuss it later.

98. As will be shown later, the multi-fold contributions associated to EPR would still amount to gravity (i.e. be associated to quanta of spacetime). Even such an approach would be worth investigating further to decide if variations or coexistence of these models would make sense.

99. And at the center of mass of EPR entangled particles.

100. Attempts to shakily extrapolate to non-anti de sitter spaces have been usually not too rigorous (for example: [309, 14]); sometimes with success but often with controversies and mistakes.

101. As well as an entropy area law for suitable surfaces in the spacetime of $U_{B G}$.

102. This is just to discuss the breadth of possibilities that have opened. With these, we are not necessarily suggesting that this is what happens. But it would be interesting to see where that thought process would lead Physics and superstrings.

103.e.g., for M-Theory.

104. We are not saying that it is what happens. We are just exploring what may be worth looking at in the context of superstrings considering what we derived in $U_{M F}$.

105.i.e. within the wave function.

106. This is a key aspect missing in the ER=EPR conjecture, and related work.

107. Which may be more rapidly falsifiable, explains the principle of equivalence, and the attraction-only property of gravity.

108. Rotating non-point particles are of course alternatives. As no quantification of folds has taken place this is not a good candidate to explain why only certain quantized spin exist and why, considering the multi-fold mechanisms, only certain quantized spin exist. Although, discreteness of the folds may lead to the answer. (Of course conventional properties of rotation symmetry group (SU(2)) suffice for this).

109.In fact, it was an inspiration for the Englert-Brout-Higgs-Guralnik-Hagen-Kibble mechanism.

110. They can be found by searching the internet.

111. Note that it's for a helicity +/- 1, or spin-1 graviton, so one should be careful about what it means exactly [304].

112. However, many related works do not seem to be in mainstream physics, e.g., too much focused on anti-gravity.

113. We do not try to argue if the Big Bang is defined as including the inflation, or, hot, post inflation. It is a question of definition.

114.Later, we will also derive GR from Statistical Physics/Thermodynamics considerations of our model.

115.Indeed, do not forget that the area /deficit angle etc. from Regge Calculus, used by the reconstructive methods, are actually the result of discretizing the Hilbert-Einstein Action [23] and/or extremizing the area (making it invariant).

116. Superstrings only recover it for exotic black holes in exotic spaces (i.e. AdS).

117.à la $[\underline{307}, \underline{376}, \underline{375}]$, which showed that such a model can be consistent with a massive quantization of GR, but in our case with expected very small scale range (For massive virtual particles), instead of the large scale considered in 
these references, and in addition to the massless gravity contribution of conventional (quantized) GR. EPR entangled massive particles, and macroscopic entanglement, as in quantum matter effects extend to the range of the entangled particles; but it is a phenomenon that is in addition to gravity.

118. Down to quantum scales, where quantum Physics applies; not down to Planck scales, as we will proposed later.

119.Emerging, entanglement, etc.

120.This is why it is useful to separate the EPR Lagrangian from gravity terms.

121. The reason being that, in Minkowski space, we can rely on straight lines between particles and illustrate more easily the reasoning. We believe that in general the reasoning can be extended to other initial conditions by following geodesics. However, we do want to avoid problems of assumptions that may not always exist when bringing in geodesics, Killing fields, proper times and suitable observers. Our initial conditions, cover all cases with positive curvature. Negative curvature would require a negative curvature initial condition and we cannot escape these challenges. However, most of the theorems below are proven in the literature for most similar conditions (with maybe a few more limitations). So we believe it is not that important to explicitly extend the proofs to $\sim \operatorname{AdS}(4)$ (and other dimensions), and rather assume that the theorems also apply there. As AdS spacetime as solution of Einstein GR field equations is unstable with matter, always resulting into black holes, it does not seem a critical issue [451].

122.It was also already established with the reference [171].

123. With the microscopic torsion generated, where matter / energy is, or the already announced discreteness of spacetime.

124. Assuming a static black hole, not accounting for charge and rotation.

125. Massive in the sense that they can no more "move forward" at $c$.

126. and absolutely no supra luminosity.

127.Light neutrino contributions can probably be considered, in first approximation, as lining up with the massless effects. It is all the other virtual particles that matter.

128. The stationary consideration at the surface mentioned earlier is key for this proof to hold, without involving light sheets.

129. Intuitively, because for the entropy of gravity, it amounts as if the coupling constant $\mathrm{G}$ increased due to the additional massive effects.

130. Definitively, for virtual particles surrounding particles.

131.That minimum distance is not that interesting if the reader can believe that we will later motivate a discrete spacetime.

132. Charged, and / or rotating, black holes have more complicated models with multiple horizons and in some situations possibly naked singularities, although not in $U_{M F}$, because of torsion and discreteness. A lot of literature exists on the subject. Entry points can be found in: $[\underline{381}, \underline{380}]$.

133.In $U_{M F}$, the increased small scale coupling due to massive effects implies a more compact set of horizons that what is discussed in [387], therefore resolving the singularity issues even if discreteness, expansion, and torsion avoid the issues.

134. because of torsion, and / or discreteness of spacetime.

135. Besides the macroscopic and other entanglement work discussed earlier and in upcoming sections on more falsifiability and validation opportunities.

136. At very small scales, yet larger than Planck scales, and also with the massive contributions.

137. The mass of the neutrinos is a bit more complex to explain, as the absence of observed right-handed neutrinos and left-handed anti neutrinos implies no mass acquisition from the Higgs mechanism [281], and multiple theories exist to explain their mass [400]. We will discuss some aspects later.

138. Here, not considering the EPR terms.

139. Massive gravity effects and EPR do not change that conclusion.

140. See footnote 170 . 
141. That paper still assumed massless neutrinos, but this is certainly aligned with a Standard Model state before considering the oscillations that introduce masses.

142. Another way to intuitively understand this is that, in the presence of gravity and uncertainties, a charge appears as a current, with preferred directions (defined by the geodesics, without them (i.e., when there is no gravity and geodesic are isotropic), uncertainties just increase the ball of the charge density, and no symmetry breaking occurs, while in their presence, currents appear for all charges), while a current remains appearing as a current possibly deformed: symmetry is broken for Maxwell equations with sources, between magnetic and electric fields. As a result, polarity (helicity or spin) is no more conserved, flips occur, just as for massive and massless fermions, or for the later spacetime orientation flips, and the arguments for symmetrizing the equations, by adding a magnetic charge density, disappear. Spacetime orientation flips will be discussed along with gravity electroweak symmetry breaking in future paper. Watch $[\underline{513}, \underline{514}, \underline{515}, \underline{516}]$.

143. We also left the door open to folds defined by Einstein's GR equations or variations, and so, for example, the folds could be wormholes or black holes as in ER=EPR.

144. By opposition to Planck scales.

145.For now, let us park aside the fact that uncertainty /vacuum noise will always create at least virtual particles. But the idea is that, if spacetime has not been concretized, it does not exist, and if it does not exist, it does not host vacuum excitations, and so virtual particles also do not appear there; noting that "there" is meaningless, in such cases.

146. Related to entropy and entanglement.

147.For example, as entanglement takes place, the dip could be thought as the manifestation of the multi-folds (think of effective curvature), or attractive potential (think of wrinkling, or dipping spacetime, as a result), or even the make of the entry point and mappings to the folds (think of trying to dig out of spacetime into the tangent dual space).

148. Something analogous to the spin network assumptions [25].

149.Entanglement and gravity are captured in the black hole energy levels and multi-folds, even if these are not exposed explicitly in Makela's work. His main recovery of GR is purely based on thermodynamics [192].

150.It is also central to the respect of the uncertainty principles by Path Integrals [247].

151. More hints, or intuition, on “why Path Integrals and Action principles?” will be provided later.

152.It is therefore not affected by astronomical results that would not support expanded ranges of fractal scales [412], beyond a homogeneity scale $(\sim 70 \mathrm{Mpc})$. Note that this latter reference is not in agreement with earlier results obtained with different methodologies as reported in $[\underline{413}, \underline{414}]$, but considered as probably incorrect.

153. When geometry is non-commutative, we have uncertainty between spatial variables: measuring a coordinate introduce uncertainties in the others and the measurements are not independent.

154. The reader may also be interested in another provocative angle, that we will not exploit, or rely upon here, in [493].

155. Yes, in fact, the attractive direction captures the contribution to the Ricci tensor of the underlying GR equations; but to see that, we would need to dive into their derivation.

156. And so, let us say it again, this may explain why semi classical models can remain valid till very small scales corresponding to the scale of the horizon of these microscopic blackholes.

157. "quasi”, because no more conventional in a discrete spacetime. For examples, singularities are no longer issues. This is why we have, and will continue to, sometimes, use this terminology of (quasi) (microscopic) black hole.

158. We will see in an upcoming section that splits of blackhole into two blackholes (in this case a charged extremal one and a minimum one) are not issues. It would create, or concretize spacetime, and use energy. Where spacetime is already concretized, no energy is involved.

159. Related, but more as something that we should keep in mind, [485] suggested that such a structure appears as strings on a black hole horizon and the horizon spacetime manifold seems to be the world sheet (or D-brane) built on these strings. In our view, this is because 't Hooft uses a similar approach as ours in section 7.4, and, as a result, he sees a horizon with its uncertainty fluctuations. Particles, or microscopic structures, as we envisage at this time, i.e., discrete spacetime nodes, seen from the outside (or inside), will appear to move along the radial axis, giving them this linear or stringy appearance deducted by 't Hooft. That does not make the structure strings or superstrings. In fact, it is 
again the result of the coincidence of Nambu-Goto Action that on such a fluctuating horizon matches an Action of area conservation (Think about a Black Hole horizon, ...), as does the Hilbert Einstein Action.

160.It may be worth repeating that this big bounce feature of all the LQG derived models are fundamentally the result of working with Actions, Lagrangians and Hamiltonians, that incorporate torsion. We know that introducing torsion [354] eliminates singularities. The big bounce could be the result of that aspect of the model; we do not start from scratch but from an initial non-singular distribution of points.

161.Energies were large at that time, so that every perturbation can create both spacetime, and particles.

162. The time fractionality follows [211] vision, but it also takes into account challenges, as described in [480].

163. We discussed already why it is not likely that it be with negative curvature.

164. And, as a side note, it is not 3-D because curvature induced gravity does not exist in 2-D space nor is Huygens principle working in 2-D space or in 4-D space (even dimensions). More dimensions (not compacted) are also simply too large for the energy content of the universe. Compacted dimensions introduce ghost, instabilities and new fields to explain. Find more in comments at [513].

165.In our view, gravity effects, and UU, prevent GUT, and Magnetic monopoles, to ever have an option to reign in $U_{M F}$; that it be at the dawn of the universe, or after, at very small ranges somewhere above Planck scales.

166. Granted also, that less known GUTs exist that avoid entirely the inertial proton decay problem. A review list is maintained at [482].

167.Discovering UU, while tinkering with the weak gravity conjecture, was quite a surprise.

168. Spin must be 0 or 1 .

169. Which are also fermion, not bosons; so they do not fit the bill. Supersymmetry would associate bosons, as super partners to the fermions (and conversely), and would provide the missing particles.

170.There is a discrepancy for massless fermions between [405] and [507]; but we argue that, in $U_{M F}$, the validation of semi classical approaches till very small scales and the presence of massive gravity gives more weight to the conclusion of [405]. If not actually due to flips of chirality, above electroweak symmetry breaking, spacetime orientation flips are also caused by gravity. 144. Find more in subsequent papers at [513].

171. From a mathematical point of view.

172. The reasoning can be repeated in the presence of gravity without using our model; but the strength of the gravity interactions needed to achieve flips and smearing may not be sufficient if semi-classical is no more valid or without massive gravity contributions at these very small scales.

173. The other dimensions of (superstrings) being in $S^{5}(+1)$ are decoupled for this purpose. Keep in mind: 10 dimensions for superstrings, and 11 for M-theory.

174. Key to variant formulations of GR, à la Einstein-Cartan with spin connections, and, hence, usually introduced when dealing with fermions and torsion.

175. We do not try to argue here if the terminology of the Big Bang should be before, or after, or as part of the inflation assuming that inflation took place.

176. $\left(\frac{d N_{\text {points }}}{d t}\right)=\alpha t$

177.or $(1 / r)^{2}$ as contribution of individual folds.

178. Depending on the physics within $\operatorname{AdS}(5)$, i.e., GR, or something else.

179. Teleportation, time travel, travel close to speed of light, antigravity, etc.

180.There is a reason why we waited the end to discuss this. 